\title{
Imaging of tumor hypoxia with PET
}

Citation for published version (APA):

Zegers, C. M. L. (2016). Imaging of tumor hypoxia with PET: a step towards individualized cancer treatment. [Doctoral Thesis, Maastricht University]. Maastricht University. https://doi.org/10.26481/dis.20160309cz

Document status and date:

Published: 01/01/2016

DOI:

$10.26481 /$ dis.20160309cz

Document Version:

Publisher's PDF, also known as Version of record

\section{Please check the document version of this publication:}

- A submitted manuscript is the version of the article upon submission and before peer-review. There can be important differences between the submitted version and the official published version of record.

People interested in the research are advised to contact the author for the final version of the publication, or visit the DOI to the publisher's website.

- The final author version and the galley proof are versions of the publication after peer review.

- The final published version features the final layout of the paper including the volume, issue and page numbers.

Link to publication

\footnotetext{
General rights rights.

- You may freely distribute the URL identifying the publication in the public portal. please follow below link for the End User Agreement:

www.umlib.nl/taverne-license

Take down policy

If you believe that this document breaches copyright please contact us at:

repository@maastrichtuniversity.nl

providing details and we will investigate your claim.
}

Copyright and moral rights for the publications made accessible in the public portal are retained by the authors and/or other copyright owners and it is a condition of accessing publications that users recognise and abide by the legal requirements associated with these

- Users may download and print one copy of any publication from the public portal for the purpose of private study or research.

- You may not further distribute the material or use it for any profit-making activity or commercial gain

If the publication is distributed under the terms of Article $25 \mathrm{fa}$ of the Dutch Copyright Act, indicated by the "Taverne" license above, 
Imaging of tumor

hypoxia with PET

a step towards individualized cancer treatment

Karen Zegers 


\section{Production}

Printing: Datawyse | Universitaire Pers Maastricht ISBN: 9789461595379

(C) Copyright C.M.L. Zegers, Maastricht 2016 


\title{
Imaging of tumor hypoxia with PET: a step towards individualized cancer treatment
}

\author{
Proefschrift \\ ter verkrijging van de graad van doctor aan de Universiteit Maastricht, \\ op gezag van de Rector Magnificus Prof. Dr. L.L.G. Soete, \\ volgens het besluit van het College van Decanen, \\ in het openbaar te verdedigen \\ op woensdag 9 maart 2016 om 14:00 uur \\ door \\ Catharina Maria Louise Zegers
}

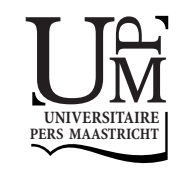




\section{Promotor}

Prof. Dr. P. Lambin

\section{Copromotoren}

Dr. W. van Elmpt

Dr. L. Dubois

\section{Beoordelingscommissie}

Prof. Dr. F. Ramaekers (Maastricht University, voorzitter)

Prof. Dr. M. Vooijs (Maastricht University)

Prof. Dr. D. Zips (University Tübingen)

Dr. J. Bussink (Radboud University Medical Centre Nijmegen) 


\section{CONTENTS}

\section{Introduction}

Chapter 1 Introduction and outline of the thesis

Chapter 2 Review: Current preclinical and clinical applications of hypoxia PET imaging using 2-nitroimidazoles

\section{Imaging of tumor hypoxia}

Chapter 3 A comparative study of the hypoxia PET tracers $\left[{ }^{18} \mathrm{~F}\right] \mathrm{HX} 4$,

Chapter $4 \quad$ Repeatability of hypoxia PET imaging using $\left[{ }^{18} \mathrm{~F}\right] \mathrm{HX} 4$ in lung and head and neck cancer patients

Chapter 5 Hypoxia imaging with $\left[{ }^{18} \mathrm{~F}\right] \mathrm{HX} 4$ PET in NSCLC patients: defining optimal imaging parameters

\section{Clinical potential and therapeutic targets}

Chapter 6 In vivo quantification of hypoxic and metabolic status of NSCLC using $\left[{ }^{18} \mathrm{~F}\right] \mathrm{HX} 4$ and $\left[{ }^{18} \mathrm{~F}\right] \mathrm{FDG}$ PET/CT imaging

Chapter $7 \quad$ Imaging of tumor hypoxia and metabolism in patients with HNSCC

Chapter 8 Evaluation of tumor hypoxia during radiotherapy using $\left[{ }^{18} \mathrm{~F}\right] \mathrm{HX} 4 \mathrm{PET}$ imaging and blood biomarkers in HNSCC

Chapter $9 \quad$ TH-302 in combination with radiotherapy enhances the therapeutic outcome and is associated with pretreatment $\left[{ }^{18} \mathrm{~F}\right] \mathrm{HX} 4$ PET imaging.

\section{Discussion}

Chapter 10 General discussion and summary

Chapter 11 Concluding remarks and future perspectives 

INTRODUCTION 



\section{CHAPTER}

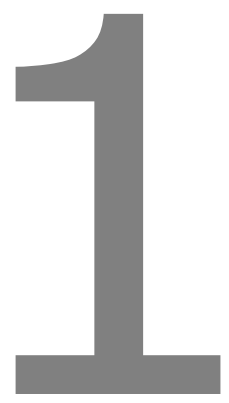

Introduction and outline of the thesis 



\section{INTRODUCTION}

\section{Cancer}

Cancer is one of the leading causes of death worldwide. The number of new cancer cases is expected to increase from 14 million in 2012 to 22 million within the next 20 years (1). Cancer originates from normal cells that undergo several genetic alterations, leading to a malignant, uncontrolled cell growth. There are more than 100 distinct types of cancer, which have various essential alterations in common. These so-called hallmarks of cancer are (i) independence in the production of growth signals, (ii) insensitivity to signals that inhibit growth, (iii) escape from programmed cell death, (iv) unlimited replicative potential, (v) sustained angiogenesis (vi) the ability to invade other tissues and metastasize, (vii) reprogramming of cellular energy metabolism and (viii) avoiding immune destruction. Due to the combination of these hallmarks, cancer cells are able to circumvent the anticancer defense mechanism of normal cells $(2,3)$.

The ultimate aim of anti-cancer therapy is to provide a personalized treatment based on patient-specific tumor characteristics. The three main cancer treatment modalities are surgery, radiotherapy and chemotherapy. Where surgery physically removes the malignant lesions, radiotherapy will deposit a high local dose of radiation to the tumor with the aim to kill all present tumor cells. Systemic chemotherapy on the other hand, targets the cancer cells based on their high proliferative characteristics. Frequently these methods are combined to provide the optimal anti-cancer treatment for each specific tumor site, taking into account the effects on healthy tissues and potential morbidity.

\section{Tumor hypoxia}

The tumor microvasculature is characterized by an impaired and chaotic blood vessel network. These blood vessels are known to be highly irregular, tortuous, have arteriovenous shunts and blind ends. In addition these vessels might collapse, causing a (temporary) occlusion. As a result, the supply of oxygen to tumor cells can be impaired, leading to regions of hypoxia (Figure 1.1) (4). The presence of hypoxic tumor cells has a negative impact on cancer treatment effectiveness, irrespective of the chosen treatment modality (5). It increases the metastatic potential, promotes resistance to treatment and is therefore related to a poor prognosis (5-8). 
12 |Chapter 1

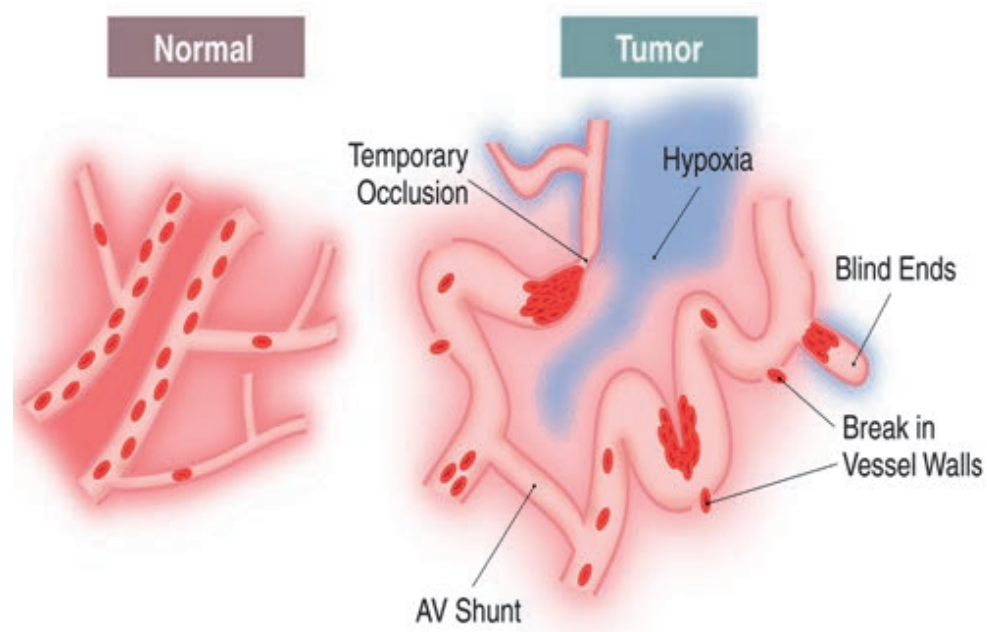

Figure 1.1: Schematic representation of the vasculature in normal and malignant tissues. [Reproduced from Brown and Giaccia (4)]

Tumor hypoxia influences malignancy through several mechanisms. The tumors adapt to a low oxygen concentration by the activation of several physiological responses, for example an increased anaerobic glycolysis and angiogenesis. Also, a natural selection takes place, since only cells that are able to cope with the low oxygen concentration will survive and proliferate, causing a more malignant phenotype. In addition hypoxia can alter the DNA repair capacity, which promotes genomic instability and can accelerate the cancer development (9).

For radiotherapy, the presence of oxygen has a beneficial value. DNA damage is induced directly or indirectly by the formation of free radicals after irradiation. These radicals are unstable and will react rapidly with oxygen to produce a stable chemical composition. The damage to the DNA is chemically 'fixed' $(5,9)$. This reaction cannot take place in the absence of oxygen, since the ionized target molecules are able to repair themselves. The extent of the DNA damage is therefore dependent on the presence or absence of oxygen. The ratio of radiation dose which will kill the same number of hypoxic cells, in comparison to normoxic cells is called the oxygen enhancement ratio and is in general around 3 (9). Theoretically, the radiation dose to hypoxic tumors should be increased to effectively treat these lesions. However, the dose to the whole tumor cannot be increased without consequences regarding healthy tissue toxicity. Smart solutions, targeting the hypoxic tumor cells (i.e. providing only a local high dose) or enhancing the susceptibility of hypoxic tumor cells to therapy (i.e. by anti-hypoxia targeting) have the potential to increase the therapeutic window of radiotherapy. However, to target tumor hypoxia, an accurate and reliable detection and visualization of tumor hypoxia is needed (10). 


\section{PET imaging}

Positron Emission Tomography (PET) is a non-invasive imaging technique, which uses radioactive labeled molecules (tracers) to visualize specific molecular interactions within the body. The radionuclide will decay, emitting a positron which reacts with a nearby electron, producing 2 photons which are emitted opposite to each other. After intravenous injection, the tracer will distribute through the body and accumulate in certain regions (e.g. the tumour), the PET scanner detects the emitted gamma photons and, after image reconstruction, visualizes the location of the radioactive tracer within the body (Figure 1.2). In general the PET scanner is combined with a computed tomography (CT) scanner to visualize the anatomy of the patient and to correct for the attenuation of the gamma photons $(11,12)$.

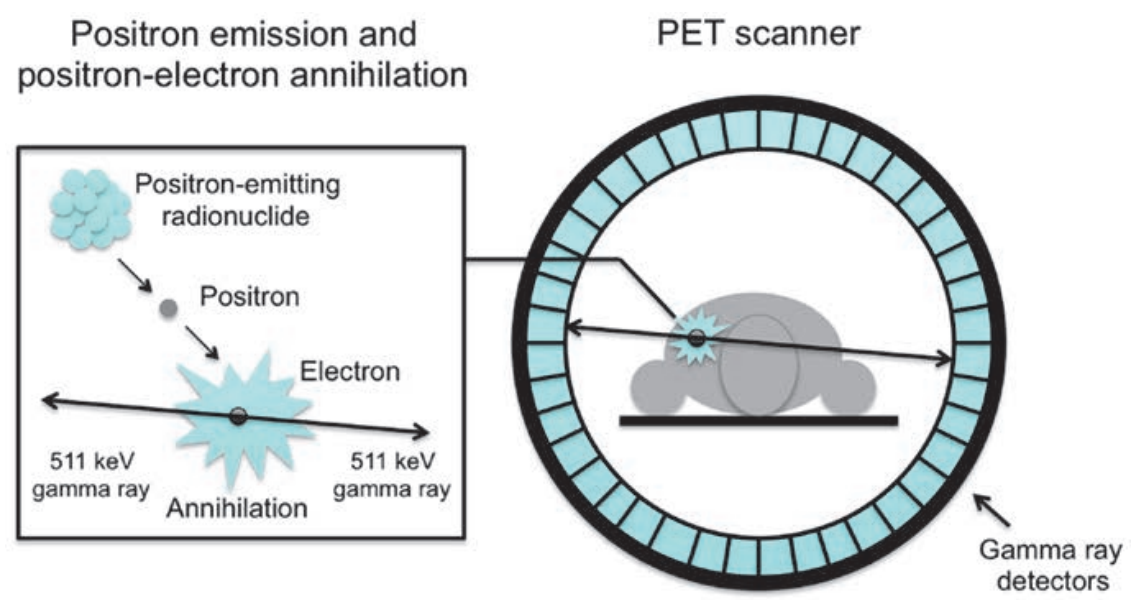

Figure 1.2: Schematic illustration of the detection of a radioactive tracer within the body. Positrons released from the radionuclide react with electrons in the tissue, releasing two gamma photons which can be detected by the PET scanner [Reproduced from van der Veldt et al. (11)].

\section{Metabolic imaging}

The most frequently used PET tracer is the Fluor-18 $\left[{ }^{18} \mathrm{~F}\right]$ labeled glucose analog, fluorodeoxyglucose (FDG). Organs with a high glucose metabolism like the brain, heart and cancer heavily consume this tracer. In contrast to normal cells, which rely primarily on oxidative phosphorylation to generate energy, most cancer cells produce energy by a high rate of aerobic glycolysis, the 'Warburg effect' (13). This upregulation of glycolysis results in an increased glucose consumption, which can be visualized with $\left[{ }^{18}\right.$ F]FDG PET imaging (14). $\left[{ }^{18}\right.$ F]FDG PET is used to detect and stage malignancies, guide radiotherapist in the delineation of the target volume and to monitor the response to treatment. In addition, previous studies have shown that the subvolumes of the tumor with the highest FDG PET uptake, were more susceptible to develop a local recurrence after (chemo)radiotherapy (15-18). Therefore, ongoing clinical studies are initiated to investigate whether a higher radiation dose to the high FDG subvolumes will increase the local tumor control (19-21). 


\section{Hypoxia imaging}

Hypoxia PET imaging is an interesting research topic for several years, but has not reached the routine clinical practice. The majority of hypoxia PET tracers are based on radioactive $\left[{ }^{18} \mathrm{~F}\right]$ labeled 2-nitroimidazoles, which are bioreduced in hypoxic tumor cells and therefore specifically bound in these cells. There are many tracers available to visualize tumor hypoxia $(10,22)$. The first hypoxia PET tracer was $\left[{ }^{18} \mathrm{~F}\right]$ misonidazole (FMISO), which was followed by the radioactive labeled etanidazole radiosensitizers ( $\left[{ }^{18} \mathrm{~F}\right] \mathrm{EF} 3$ and $\left[{ }^{18} \mathrm{~F}\right] \mathrm{EF} 5$ ). These three hypoxia PET tracers have a relatively high lipophilicity, which allows them to easily penetrate the cell membranes, but therefore show a slow clearance of unbound tracer from the normal tissues. This causes a low image contrast between the hypoxic tumor and normal tissues. Therefore, more hydrophilic PET tracers were developed, to allow a faster clearance from the non-hypoxic tissues, with the aim to obtain a higher image contrast. Examples of these hydrophilic PET tracers are fluoroerythronitroimidazole $\left(\left[{ }^{18} \mathrm{~F}\right] \mathrm{FETNIM}\right)$ and fluoroazomycin arabinoside $\left(\left[{ }^{18} \mathrm{~F}\right] \mathrm{FAZA}\right)$. The most recently developed, very hydrophilic PET tracer is flortanidazole $\left[{ }^{18} \mathrm{~F}\right] \mathrm{HX} 4$, which aims for preferred pharmacokinetics and clearance properties. $\left[{ }^{18} \mathrm{~F}\right] \mathrm{HX} 4$ was used in preclinical setting in a rat rhabdomyosarcoma model. In this model the uptake of $\left[{ }^{18} \mathrm{~F}\right] \mathrm{HX} 4$ was significantly correlated to the immunohistochemistry staining for hypoxia (pimonidazole), providing the evidence that $\left[{ }^{18} \mathrm{~F}\right] \mathrm{HX} 4$ accumulates specifically in the hypoxic subvolumes of the tumor (23). The biodistribution and radiation dosimetry of $\left[{ }^{18} \mathrm{~F}\right] \mathrm{HX} 4$ was assessed in monkeys and humans, showing that the effective dose of $\left[{ }^{18} \mathrm{~F}\right] \mathrm{HX} 4$ PET was comparable to other PET imaging modalities with the $\left[{ }^{18} \mathrm{~F}\right]$ radionuclide (24). The first clinical phase I study with $\left[{ }^{18} \mathrm{~F}\right] \mathrm{HX} 4$ proved the feasibility and safety of $\left[{ }^{18} \mathrm{~F}\right] \mathrm{HX} 4$ PET in patients without toxicity (25). Based on these promising results, new preclinical and clinical studies were initiated using $\left[{ }^{18} \mathrm{~F}\right] \mathrm{HX} 4$ PET imaging. The current status of hypoxia PET imaging with $\left[{ }^{18} \mathrm{~F}\right] \mathrm{HX} 4$ will be presented in the next chapters of this thesis.

\section{Lung and head \& neck cancer}

The use of radioactive labeled nitroimidazoles to assess tumor hypoxia by PET imaging is described for various carcinomas. The literature is however, dominated by studies in patients with head and neck cancer and lung cancer. In 1996 Rasey et al. already described the quantification of tumor hypoxia, using $\left[{ }^{18} \mathrm{~F}\right] \mathrm{FMISO}$, in patients with non-small-cell-lung cancer (NSCLC) and head and neck cancer. They observed tumor hypoxia in all NSCLC and head and neck cancer lesions, however a broad range $(0.2-94.7 \%)$ of the fractional hypoxic volume was reported (26). Also, more recent studies confirm that in the majority of head and neck cancer and lung cancer patients a significant amount of tumor hypoxia is present, which can counteract the treatment effectiveness in these patients (7, 27-29). The meta-analysis published by Overgaard et al. $(30,31)$ showed that hypoxia modifying treatment has the potential to improve tumor control in patients with head and neck cancer. In addition, in patients with locally advanced non-small cell lung cancer (NSCLC) there is a need to improve the tumor control, since the standard treatment modality (radiotherapy in combination with chemotherapy), has a limited progression free survival (32). Therefore, the focus of this thesis will be the use of hypoxia PET imaging in patients with head and neck and lung cancer. 


\section{OBJECTIVE AND OUTLINE OF THE THESIS}

The general objective of this thesis is to show that non-invasive imaging of tumor hypoxia has the potential to contribute to individualized anti-cancer treatment by (i) the accurate and reliable identification of hypoxic (sub)volumes within the tumor and (ii) the ability to monitor the response to treatment.

In Chapter 2 a literature overview is presented including preclinical and clinical research using 2-nitroimidazole based hypoxia PET tracers. Topics regarding the validation and quantification of hypoxia PET imaging and its (clinical) applications are addressed. The next three chapters are focused on the ability to select the optimal hypoxia tracer and its implementation in clinical practice. Chapter 3 describes a pre-clinical study comparing frequently used 2-nitroimidazole based hypoxia PET tracers FAZA, FMISO and HX4 within one tumor model. In Chapter 4 the repeatability of the hypoxia PET tracer HX4 is addressed in patients with head and neck and lung cancer. The focus of Chapter 5 is the definition of optimal imaging parameters for HX4 PET imaging in patients with non-small cell lung cancer (NSCLC). The second part of this thesis shows the clinical results of HX4 PET imaging and its potential use for therapeutic targeting. Chapter 6 and Chapter 7 evaluate the added value of hypoxia PET imaging with HX4 to the frequently used metabolic PET tracer FDG in patients with NSCLC and head and neck cancer, respectively. Chapter 8 provides insight in the changes of tumor hypoxia during treatment in patients with HNSCC. In addition the link between imaging and blood biomarkers is addressed. In Chapter 9 the results are presented of the addition of the hypoxia targeted drug $\mathrm{TH}-302$ to radiotherapy in combination with hypoxia PET imaging. In Chapter 10 a general discussion and summary are provided. Finally, in Chapter 11 the concluding remarks and future perspectives on the topics presented within this thesis are discussed. 


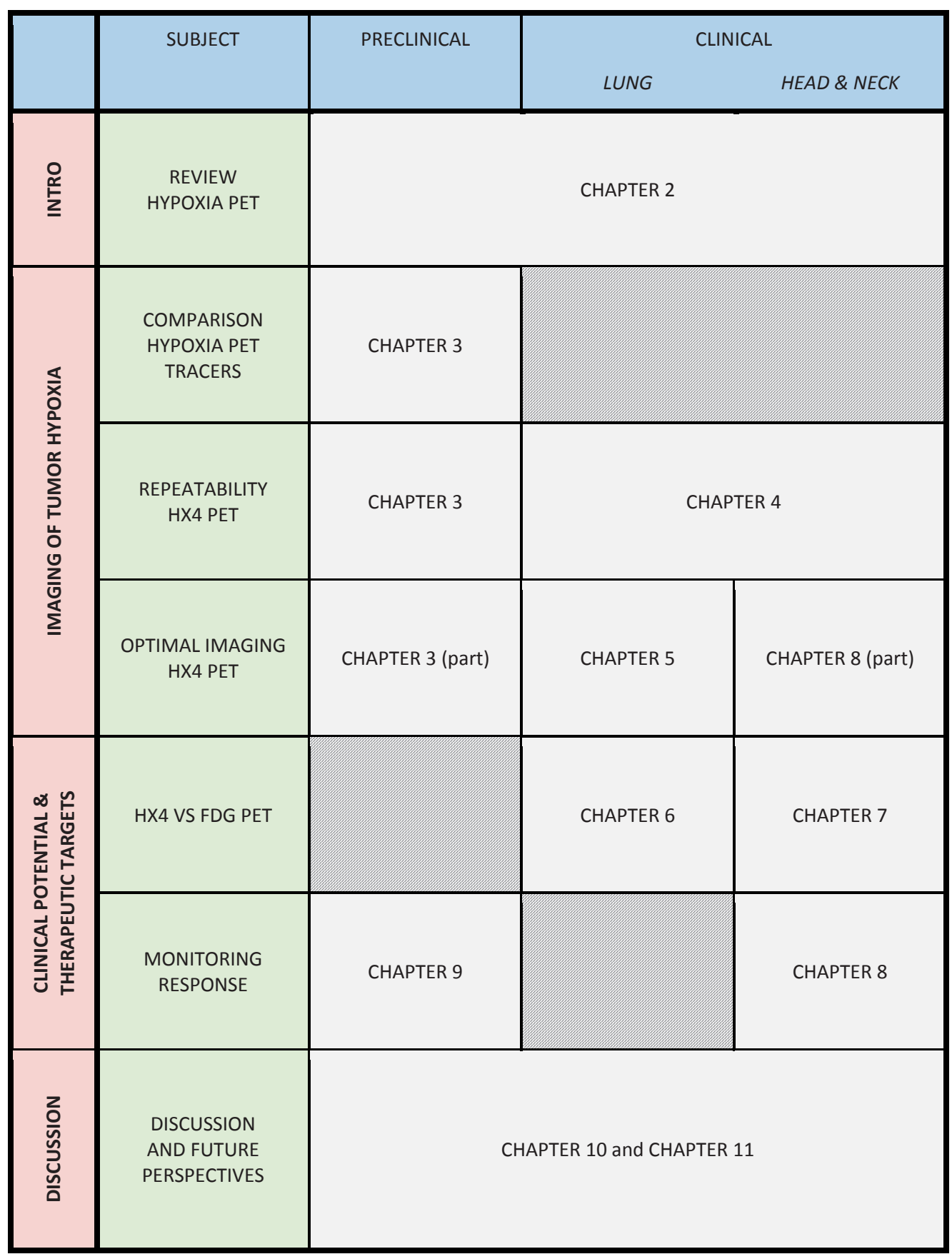




\section{REFERENCES}

1. World Health Organisation. February 2015 [Available from: http://www.who.int/ mediacentre/factsheets/fs297/en/]

2. Hanahan D, Weinberg RA. The hallmarks of cancer. Cell. 2000;100:57-70.

3. Hanahan D, Weinberg RA. Hallmarks of cancer: the next generation. Cell. 2011;144: 646-74.

4. Brown JM, Giaccia AJ. The unique physiology of solid tumors: opportunities (and problems) for cancer therapy. Cancer Res. 1998;58:1408-16.

5. Pettersen EO, Ebbesen P, Gieling RG, Williams KJ, Dubois L, Lambin P, et al. Targeting tumour hypoxia to prevent cancer metastasis. From biology, biosensing and technology to drug development: the METOXIA consortium. J Enzyme Inhib Med Chem. 2014:1-33.

6. Nordsmark M, Bentzen SM, Rudat V, Brizel D, Lartigau E, Stadler P, et al. Prognostic value of tumor oxygenation in 397 head and neck tumors after primary radiation therapy. An international multi-center study. Radiother Oncol. 2005;77:18-24.

7. Zips D, Zophel K, Abolmaali N, Perrin R, Abramyuk A, Haase R, et al. Exploratory prospective trial of hypoxia-specific $\mathrm{PET}$ imaging during radiochemotherapy in patients with locally advanced head-and-neck cancer. Radiother Oncol. 2012;105:21-8.

8. Milosevic M, Warde P, Menard C, Chung P, Toi A, Ishkanian A, et al. Tumor hypoxia predicts biochemical failure following radiotherapy for clinically localized prostate cancer. Clin Cancer Res. 2012;18:2108-14.

9. Joiner M, van der Kogel A. Basic Clinical Radiobiology. 2009.

10. Horsman MR, Mortensen LS, Petersen JB, Busk M, Overgaard J. Imaging hypoxia to improve radiotherapy outcome. Nat Rev Clin Oncol. 2012;9:674-87.

11. van der Veldt AA, Smit EF, Lammertsma AA. Positron Emission Tomography as a Method for Measuring Drug Delivery to Tumors in vivo: The Example of $[(11) C]$ docetaxel. Front Oncol. 2013;3:208.

12. Gambhir SS. Molecular imaging of cancer with positron emission tomography. Nat Rev Cancer. 2002;2:683-93.

13. Vander Heiden MG, Cantley LC, Thompson CB. Understanding the Warburg effect: the metabolic requirements of cell proliferation. Science. 2009;324:1029-33.

14. Gatenby RA, Gillies RJ. Why do cancers have high aerobic glycolysis? Nat Rev Cancer. 2004;4:891-9.

15. Aerts HJ, van Baardwijk AA, Petit SF, Offermann C, Loon J, Houben R, et al. Identification of residual metabolic-active areas within individual NSCLC tumours using a pre-radiotherapy (18)Fluorodeoxyglucose-PET-CT scan. Radiother Oncol. 2009;91:386-92.

16. Due AK, Vogelius IR, Aznar MC, Bentzen SM, Berthelsen AK, Korreman SS, et al. Recurrences after intensity modulated radiotherapy for head and neck squamous cell carcinoma more likely to originate from regions with high baseline [18F]-FDG uptake. Radiother Oncol. 2014;111:360-5.

17. Calais J, Thureau S, Dubray B, Modzelewski R, Thiberville L, Gardin I, et al. Areas of high 18F-FDG uptake on preradiotherapy PET/CT identify preferential sites of local 
relapse after chemoradiotherapy for non-small cell lung cancer. J Nucl Med. 2015;56:196-203.

18. Calais J, Dubray B, Nkhali L, Thureau S, Lemarignier C, Modzelewski R, et al. High FDG uptake areas on pre-radiotherapy PET/CT identify preferential sites of local relapse after chemoradiotherapy for locally advanced oesophageal cancer. Eur J Nucl Med Mol Imaging. 2015;42:858-67.

19. Heukelom J, Hamming O, Bartelink H, Hoebers F, Giralt J, Herlestam T, et al. Adaptive and innovative Radiation Treatment FOR improving Cancer treatment outcomE (ARTFORCE); a randomized controlled phase II trial for individualized treatment of head and neck cancer. BMC Cancer. 2013;13:84.

20. van Elmpt W, De Ruysscher D, van der Salm A, Lakeman A, van der Stoep J, Emans D, et al. The PET-boost randomised phase II dose-escalation trial in non-small cell lung cancer. Radiother Oncol. 2012;104:67-71.

21. Berwouts D, Olteanu LA, Duprez F, Vercauteren T, De Gersem W, De Neve W, et al. Three-phase adaptive dose-painting-by-numbers for head-and-neck cancer: initial results of the phase I clinical trial. Radiother Oncol. 2013;107:310-6.

22. Lopci E, Grassi I, Chiti A, Nanni C, Cicoria G, Toschi L, et al. PET radiopharmaceuticals for imaging of tumor hypoxia: a review of the evidence. Am J Nucl Med Mol Imaging. 2014;4:365-84.

23. Dubois LJ, Lieuwes NG, Janssen MH, Peeters WJ, Windhorst AD, Walsh JC, et al. Preclinical evaluation and validation of [18F]HX4, a promising hypoxia marker for PET imaging. Proc Natl Acad Sci U S A. 2011;108:14620-5.

24. Doss $M$, Zhang JJ, Belanger MJ, Stubbs JB, Hostetler ED, Alpaugh K, et al. Biodistribution and radiation dosimetry of the hypoxia marker $18 \mathrm{~F}-\mathrm{HX} 4$ in monkeys and humans determined by using whole-body PET/CT. Nucl Med Commun. 2010;31:1016-24.

25. van Loon J, Janssen $M H$, Ollers $M$, Aerts $H J$, Dubois $L$, Hochstenbag $M$, et al. PET imaging of hypoxia using [18F]HX4: a phase I trial. Eur J Nucl Med Mol Imaging. 2010;37:1663-8.

26. Rasey JS, Koh WJ, Evans ML, Peterson LM, Lewellen TK, Graham MM, et al. Quantifying regional hypoxia in human tumors with positron emission tomography of [18F]fluoromisonidazole: a pretherapy study of 37 patients. Int J Radiat Oncol Biol Phys. 1996;36:417-28.

27. Bollineni VR, Wiegman EM, Pruim J, Groen HJ, Langendijk JA. Hypoxia imaging using Positron Emission Tomography in non-small cell lung cancer: implications for radiotherapy. Cancer Treat Rev. 2012;38:1027-32.

28. Mortensen LS, Johansen J, Kallehauge J, Primdahl H, Busk M, Lassen P, et al. FAZA $\mathrm{PET} / \mathrm{CT}$ hypoxia imaging in patients with squamous cell carcinoma of the head and neck treated with radiotherapy: results from the DAHANCA 24 trial. Radiother Oncol. 2012;105:14-20.

29. Grootjans W, de Geus-Oei LF, Troost EG, Visser EP, Oyen WJ, Bussink J. PET in the management of locally advanced and metastatic NSCLC. Nat Rev Clin Oncol. 2015.

30. Overgaard J, Horsman MR. Modification of Hypoxia-Induced Radioresistance in Tumors by the Use of Oxygen and Sensitizers. Semin Radiat Oncol. 1996;6:10-21. 
31. Overgaard J. Hypoxic modification of radiotherapy in squamous cell carcinoma of the head and neck--a systematic review and meta-analysis. Radiother Oncol. 2011;100:22-32.

32. van Baardwijk A, Reymen B, Wanders S, Borger J, Ollers M, Dingemans AM, et al. Mature results of a phase II trial on individualised accelerated radiotherapy based on normal tissue constraints in concurrent chemo-radiation for stage III non-small cell lung cancer. Eur J Cancer. 2012;48:2339-46. 



\section{CHAPTER}

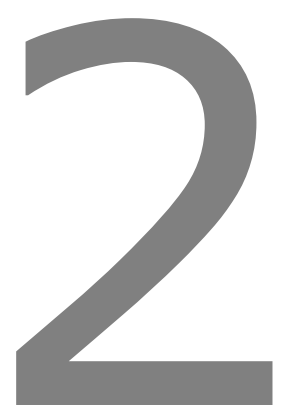

Current preclinical and clinical applications of hypoxia PET imaging using 2-nitroimidazoles

Sarah G.J.A. Peeters*, Catharina M.L. Zegers*, Ala Yaromina, Wouter van Elmpt, Ludwig Dubois and Philippe Lambin

*contributed equally

Published in: Q J NUcl Med Mol Imaging. 2015; 59(1):39-57 
22 |Chapter 2

\begin{abstract}
Hypoxia is a common characteristic of solid tumors and is associated with poor prognosis. Positron emission tomography (PET) can visualize tumor hypoxia in a non-invasive, 3dimensional manner and can be used to acquire information longitudinally. Multiple 2nitroimidazole based PET tracers are developed, validated and quantified in the search for the ideal hypoxia tracer and several tracers have shown to reliably represent tumor hypoxia. Furthermore, multiple studies describe the prognostic value of hypoxia PET imaging and the ability to monitor hypoxia during treatment. These applications can be of great potential and their role in treatment planning and modification needs to be further assessed with respect to personalized chemoradiation therapy. In this review we focus on the tracers that were positively validated in preclinical and clinical studies and report accurate quantification and visualization of hypoxia. The characteristics of these tracers are summarized for both preclinical and clinical studies. Furthermore, the clinical applications of hypoxia PET imaging are addressed with a focus on the ability to reliably monitor tumor hypoxia during treatment and the prognostic potential. Also the feasibility studies for hypoxia guided intensity modulated radiation therapy and the patient stratification for hypoxia targeted drugs are assessed.
\end{abstract}




\section{INTRODUCTION}

Hypoxia in solid tumors is a negative prognostic factor for treatment outcome. ${ }^{1}$ Due to impaired and chaotic blood vessel development, cells outgrow the blood supply or vessels collapse leading to regions of chronic and acute hypoxia. Gold standard techniques for the detection of hypoxic regions consist of measuring oxygen concentrations directly with the Eppendorf oxygen sensitive electrode or immunohistochemistry of tumor biopsies. However both techniques are invasive and do not represent the tumor in three dimensions. In contrast, hypoxia position emission tomography (PET) imaging gives the opportunity to visualize the extent of hypoxia in a non-invasive manner, in three dimensions and additionally is able to provide information longitudinally, i.e. hypoxia status can be monitored over the course of treatment. For these reasons, hypoxia PET imaging has been widely studied over the last decade and is developing from a diagnostic technique that can detect hypoxia, into a tool that can be integrated versatile, i.e. in the treatment monitoring, outcome prediction and treatment targeting. Multiple tracers have been developed, validated and shown to exhibit different characteristics. In this review we focus on nitroimidazolebased tracers that were validated in preclinical and clinical studies and report accurate and reproducible quantification of hypoxia for multiple cancer sites. We discuss the ability of these hypoxia PET tracers to monitor treatment response and address their prognostic and predictive value for treatment outcome. Furthermore we assess current and future opportunities of hypoxia PET imaging as a tool to support treatment decisions (Figure 2.1).

\section{HYPOXIA PET TRACERS}

Most PET tracers suitable for the detection of hypoxia are based on the principle of 2nitroimidazoles. After injection this molecule can diffuse freely from the vascular compartment to surrounding tissues where an electron reduction occurs which is reversed again under normal oxygen conditions. However, upon low oxygen conditions or in the presence of certain nitroreductase enzymes the molecule undergoes a second electron reduction and binds covalently to cellular components, causing the tracer to get trapped. Because of this specific accumulation in hypoxic tumor regions, and washout of the tracer in normal oxygenated tissues, these tumor areas can be clearly visualized by detection of the radioactive label connected to the tracer. The perfect hypoxia tracer should fulfill certain criteria, i.e. a large difference in washout rate between background and oxygen deprived tissues for high signal to noise ratios and specific and irreversible binding to hypoxic regions. Furthermore, the hydrophilicity of a tracer is of importance for the amount of clearance in well oxygenated tissues while more lipophilic tracers accumulate more easily in hypoxic tissues. The first developed hypoxia PET tracer based on the 2-nitroimidazole principle is $\left[{ }^{18} \mathrm{~F}\right]$ labeled fluoromisonidazole $\left(\left[{ }^{18} \mathrm{~F}\right] \mathrm{FMISO}\right)$. However, some concerns were raised about the stability of $\left[{ }^{18} \mathrm{~F}\right] \mathrm{FMISO}$, the metabolite formation ${ }^{2}$ and the slow clearance from background tissues, causing modest signal to noise ratios. ${ }^{3}$ Therefore, second generation hypoxia PET tracers have been developed to overcome these concerns. $\left[{ }^{18} \mathrm{~F}\right]$ Fluoroazomycin arabinoside $\left(\left[{ }^{18} \mathrm{~F}\right] \mathrm{FAZA}\right)$ is a nitroimidazole coupled to an arabinose sugar making the tracer more hydrophilic compared to $\left[{ }^{18} \mathrm{~F}\right] \mathrm{FMISO}$. Due to this property the visualization of the tracer should 
result in faster clearance from non-hypoxic/normal tissue and therefore have a better signal to noise ratio. $\left[{ }^{18} \mathrm{~F}\right]$ Fluoroerythronitroimidazole $\left(\left[{ }^{18} \mathrm{~F}\right] \mathrm{FETNIM}\right)$ is also a hydrophilic compound, causing higher tumor to background ratios compared to $\left[{ }^{18} \mathrm{~F}\right] \mathrm{FMISO} .{ }^{4}\left[{ }^{18} \mathrm{~F}\right] \mathrm{FETNIM}$ metabolite analysis showed high amounts of unchanged tracer present in plasma and urine of rats and dogs, whereas in the liver almost no intact $\left[{ }^{18}\right.$ F]FETNIM was present. Only low amounts of $\left[{ }^{18}\right.$ F]FETNIM binding to plasma proteins was observed. ${ }^{4}$ Tracers with a more lipophilic character were also of potential interest, leading to the development of the fluorinated etanidazole compounds $\left[{ }^{18} \mathrm{~F}\right] \mathrm{EF5}$ and $\left[{ }^{18} \mathrm{~F}\right] \mathrm{EF} 3$. EF5 was first used as a monoclonal antibody for invasive detection of hypoxia in tissue biopsies. ${ }^{5}$ In the first study describing the radioactive labeling of this 2-nitroimidazole, analysis of metabolites showed that the tracer was unmodified. ${ }^{6}$ The chemical structure of $\left[{ }^{18} \mathrm{~F}\right] \mathrm{EF} 3$, and $\left[{ }^{18} \mathrm{~F}\right] \mathrm{EF} 5$ are analogous to each other although small differences in chemical properties cause differences in biodistribution. Another tracer that tries to overcome the limitations of tumor to background ratio and aims for preferred pharmacokinetics and clearance properties is flortanidazole $\left[{ }^{18} \mathrm{~F}\right] \mathrm{HX} 4 .^{7} \mathrm{~A}$ biodistribution study of $\left[{ }^{18} \mathrm{~F}\right] \mathrm{HX} 4$ showed high uptake in the bladder, liver and kidneys with the bladder wall being the most critical organ absorbing most radiation. ${ }^{8}$ The same study shows that $82 \%$ and $84 \%$ of the $\left[{ }^{18} \mathrm{~F}\right] \mathrm{HX} 4$ is intact and unmetabolized after 2 hours p.i. in the plasma and urine, respectively. Although many more hypoxia tracers have been developed and reviewed, ${ }^{9}$ this overview focusses on the ones that have been validated successfully in preclinical and clinical studies (Figure 2.1).

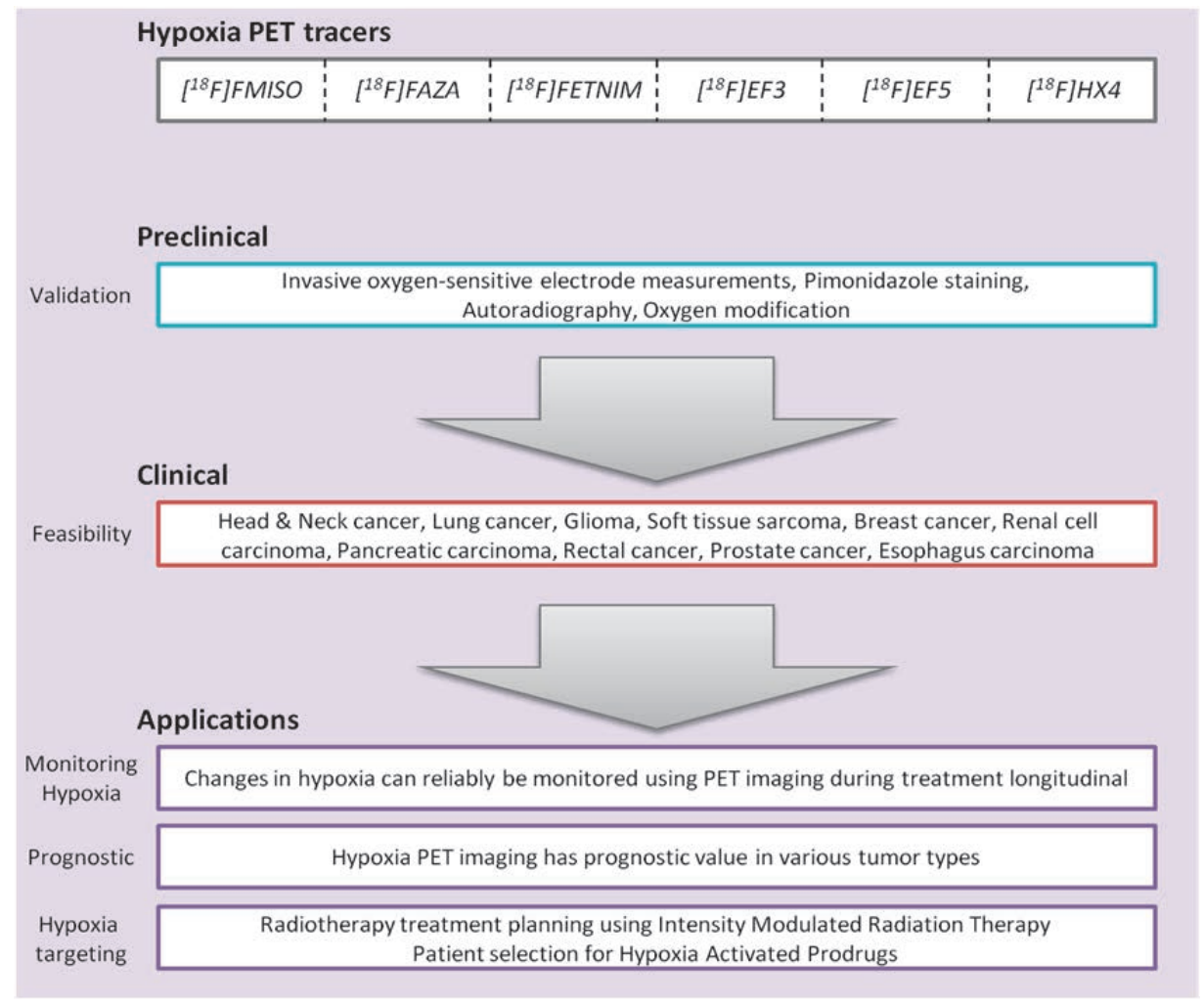

Figure 2.1: Overview of the outline of the review 


\section{VALIDATION}

An important step in the assessment of a successful hypoxia PET tracer is the validation of these agents, to ensure that the observed PET uptake in the tumor represents actual tumor hypoxia. First of all this can be done in a direct comparison between the hypoxia PET characteristics, like standardized uptake value or tumor to background ratio, and the gold standard which assesses the partial oxygen pressure in the tumor, i.e. Eppendorf $\mathrm{pO}_{2}$ measurements. In addition, hypoxia PET imaging can be correlated to immunohistochemical markers which directly or indirectly represent tumor hypoxia. Another validation method to detect hypoxia specific tracer accumulation is by exposing subjects to modified oxygen concentration breathing just before and after the tracer injection. Preclinical studies form the ideal platform for the first feasibility and validation studies. However, since pharmacokinetics differ between humans and rodents, further validation in clinical trials is necessary.

\section{$\left[{ }^{18}\right.$ F]FMISO}

The validation of $\left[{ }^{18} \mathrm{~F}\right] \mathrm{FMISO}$ has been performed in multiple studies which assessed the uptake and distribution of $\left[{ }^{18} \mathrm{~F}\right] \mathrm{FMISO}$ in different tumor models using a range of validation techniques. A selection of these studies is reported here.

The first assessment of oxygen dependency of $\left[{ }^{18} \mathrm{~F}\right] \mathrm{FMISO}$ was demonstrated in porcine livers. ${ }^{10}$ Feasibility of hypoxia imaging using $\left[{ }^{18} \mathrm{~F}\right] \mathrm{FMISO}$ was shown in tumor-bearing mice although no correlation was found with oxygen partial pressure as detected by polarographic oxygen-sensitive electrodes. However, autoradiography showed more $\left[{ }^{18} \mathrm{~F}\right] \mathrm{FMISO}$ uptake in the tumor of ambient air breathing animals compared to better oxygenated tumors in animals exposed to carbogen $\left(95 \% \mathrm{O}_{2}, 5 \% \mathrm{CO}_{2}\right)$ breathing. ${ }^{11}$ In a rat rhabdomyosarcoma tumor model a heterogeneous pattern was observed on autoradiography for both $\left[{ }^{18} \mathrm{~F}\right] \mathrm{FMISO}$ uptake and pimonidazole staining. ${ }^{12}$ Furthermore a significant correlation was observed between hypoxic volumes assessed by $\left[{ }^{18} \mathrm{~F}\right] \mathrm{FMISO}$ PET and pimonidazole immunostaining in tumor sections. A weak but significant correlation was found for 10 different human primary head and neck xenograft tumor models comparing $\left[{ }^{18} \mathrm{~F}\right] \mathrm{FMISO}$ uptake with pimonidazole staining, however, the correlation was stronger when the pattern of hypoxia was ribbon-like and had a micro-regional distribution instead of a homogeneous distribution. ${ }^{13}$ In addition a relationship between $\left[{ }^{18} \mathrm{~F}\right] \mathrm{FMISO}$ and pimonidazole was observed in Dunning R3327-AT bearing rats, although some mismatch was found possibly due to incomplete washout of $\left[{ }^{18} \mathrm{~F}\right] \mathrm{FMISO}$ from the well-perfused areas. ${ }^{14}$ Moreover, $\left[{ }^{18} \mathrm{~F}\right] \mathrm{FMISO}$ PET imaging was able to detect hypoxic micro-metastases as confirmed by autoradiography and pimonidazole staining. ${ }^{15}$ Responsiveness of $\left[{ }^{18} \mathrm{~F}\right] \mathrm{FMISO}$ to different oxygen concentrations $\left(10 \% \mathrm{O}_{2}\right.$, normal air or carbogen) was shown in a SCCVII tumor model and corresponded with pimonidazole uptake. ${ }^{16}$

Dogs bearing spontaneous sarcomas were imaged using $\left[{ }^{18} \mathrm{~F}\right] \mathrm{FMISO}$ and these data confirmed the Eppendorf $\mathrm{pO}_{2}$ measurements. ${ }^{17}$ Similar findings were observed in R3327-AT bearing rats where oxygen probe measurements broadly corresponded with $\left[{ }^{18} \mathrm{~F}\right] \mathrm{FMISO}$ 
uptake. ${ }^{18}$ However, Chang et al reported that although the overall data were positive, large variation was observed between individual data pairs indicating that $\left[{ }^{18} \mathrm{~F}\right] \mathrm{FMISO}$ PET imaging might be problematic. ${ }^{19}$ Using the combined diffusion-retention dynamic information of two $\left[{ }^{18} \mathrm{~F}\right] \mathrm{FMISO}$ scans performed at 0-15 $\mathrm{min}$ p.i. and $4 \mathrm{~h}$ p.i., reported to give an accurate measure of the median oxygen concentration comparable to average oxygen probe measurements. $^{20}$

In clinical setting, most validation studies using the Eppendorf $\mathrm{pO}_{2}$ measurements are performed in the head and neck cancer patient population, because of the relative easy accessible tumor location. Several publications correlate the $\left[{ }^{18} \mathrm{~F}\right] \mathrm{FMISO}$ PET uptake with $\mathrm{pO}_{2}$ measurements, however with contradictory results. Mortensen et al. ${ }^{21}$ observed no correlation, while Gagel et $a l^{22}$ and Zimny et $a l^{23}$ reported respectively a moderate and strong correlation. The validation of hypoxia PET imaging in 13 patients with accessible soft tissue sarcomas was performed by Bentzen et al. in combination with Eppendorf $\mathrm{pO}_{2}$ measurements. ${ }^{24} \mathrm{~A}$ lack of concordance between the PET results and the oxygen measurements was observed, suggesting that $\left[{ }^{18} \mathrm{~F}\right] \mathrm{FMISO}$ PET imaging was not able to detect tumor hypoxia or its extend as defined by $\mathrm{pO}_{2}$ measurements.

The clinical $\left[{ }^{18} \mathrm{~F}\right] \mathrm{FMISO}$ characteristics were also compared to the immunohistochemical staining of endogenous hypoxia markers. A weak but significant correlation between the $\left[{ }^{18} \mathrm{~F}\right] \mathrm{FMISO}$ hypoxic volume and HIF1 $\alpha$ expression was found in head and neck cancer patients. $^{25,}{ }^{26}$ In patients with non-small-cell-lung cancer (NSCLC) no correlation was observed between the $\left[{ }^{18} \mathrm{~F}\right] \mathrm{FMISO}$ uptake and the immunohistochemical tumor markers microvessel density, HIF1 $\alpha$, VEGF and GLUT1. ${ }^{27}$ In contrast, in patients with glioma brain tumors a significant correlation between $\left[{ }^{18} \mathrm{~F}\right] \mathrm{FMISO}$ PET imaging and the vascular endothelial growth factor receptor (VEGF) and the cell proliferation associated antigen (Ki67) expression was observed. The relationship between tumor hypoxia and Ki67 expression could be explained by the fact that both tumor parameters were related to the tumor grade. ${ }^{28}$ In addition HIF1 $\alpha$ expression showed a (non-significant) trend towards increased uptake. In breast cancer patients there was no correlation between hypoxia PET imaging using $\left[{ }^{18}\right.$ F]FMISO and HIF1- $\alpha$ expression, however, a significant correlation was observed with the proliferation marker Ki67. ${ }^{29}$ In renal cell carcinoma an increased microvessel density was present in comparison to the normal kidney parenchyma, however, this was not correlated to $\left[{ }^{18}\right.$ F]FMISO PET imaging. ${ }^{30}$

\section{$\left[{ }^{18}\right.$ F]FAZA}

Feasibility of $\left[{ }^{18} \mathrm{~F}\right]$ FAZA visualization was assessed in a squamous cell carcinoma of the head and neck ( $\mathrm{FaDu}$ ), a cervix ( $\mathrm{SiHa}$ ) tumor model and two murine tumor models. $\left[{ }^{18} \mathrm{~F}\right]$ FAZA uptake based on microPET imaging was compared to Eppendorf $\mathrm{pO}_{2}$ measurements, autoradiography and pimonidazole immunohistochemistry staining. Based on the invasive Eppendorf electrode measurements all tumors were assigned as being very hypoxic which was confirmed by pimonidazole staining and $\left[{ }^{18}\right.$ F]FAZA PET images. Furthermore significant correlations were observed between the autoradiography images and pimonidazole uptake, while overall an inverse relationship was found with the perfusion marker Hoechst 33342. ${ }^{31,32}$ These data were confirmed in the same murine tumor 
model on multiple time points post tracer injection and supplemented with biodistribution data confirming the previous findings. ${ }^{33}$ In addition $\left[{ }^{18} \mathrm{~F}\right.$ FAZA uptake was shown to correlate with the mRNA expression of CA9 and GLUT-1 after fragmenting the tumor in milligram-sized portions, which, according to the authors, allows multiple pairwise measurements at the smallest volumetric scale possible. ${ }^{34}$ Autoradiography of $\left[{ }^{18} \mathrm{~F}\right] \mathrm{FAZA}$ distribution in a SQ20b xenograft model also showed a clear positive relationship with pimonidazole and CAIX staining. ${ }^{35}$ Another validation study for $\left[{ }^{18} \mathrm{~F}\right] \mathrm{FAZA}$ uptake was performed by exposing rhabdomyosarcoma bearing rats to carbogen breathing which resulted in a decreased uptake of the hypoxia tracer. ${ }^{36}$ Moreover, $\left[{ }^{18}\right.$ F]FAZA PET correlated with the hypoxic fraction as measured by oxyLite $\mathrm{pO}_{2}$, Electron Paramagnetic Resonance (EPR) and ${ }^{19} \mathrm{~F}-\mathrm{MRI}$ suggesting that $\left[{ }^{18} \mathrm{~F}\right] \mathrm{FAZA}$ PET is a promising imaging technique for hypoxia evaluation $^{36}$ In an orthotropic brain tumor model the detection of hypoxia using $\left[{ }^{18}\right.$ F]FAZA PET imaging revealed low tumor to background ratios at 18-20 days post tumor implantation while high ratios were observed after 28-30 days. ${ }^{37}$ Bioluminescence imaging of the luciferase activity induced under the control of 'hypoxia responsive elements' confirmed that hypoxia development started around day 18 and increased from this day onward.

\section{$\left[{ }^{18}\right.$ F]FETNIM}

In preclinical setting, the paper describing the synthesis and first validation of $\left[{ }^{18} \mathrm{~F}\right] \mathrm{FETNIM}$ reports high tumor to blood (TBR) and tumor to muscle (TMR) at $4 \mathrm{~h}$ p.i. as detected by biodistribution. In addition on autoradiography hypoxic regions could be differentiated from necrotic regions. ${ }^{38}$ In NSCLC patients, Hu et al. observed a significant correlation between $\left[{ }^{18} \mathrm{~F}\right]$ FETNIM hypoxia PET imaging and the immunohistochemical markers HIF1 $\alpha$, GLUT-1 and VEGF. Since all these immunohistochemical markers are up-regulated in the absence of oxygen, this result provides the indirect evidence that $\left[{ }^{18}\right.$ F]FETNIM uptake gives an accurate indication of tumor hypoxia. ${ }^{39}$

\section{$\left[{ }^{18} \mathrm{~F}\right]$ EF5 \& $\left[{ }^{18} \mathrm{~F}\right]$ EF3}

Studies comparing $\left[{ }^{18} \mathrm{~F}\right] \mathrm{EF} 5$ with $\mathrm{pO}_{2}$ or pimonidazole assessed hypoxic fractions are not available. However, validation of $\left[{ }^{18} \mathrm{~F}\right] \mathrm{EF5}$ in a HCT116 model reported feasibility of PET acquisition based on ex vivo gamma counting. ${ }^{40}$ Feasibility of $\left[{ }^{18} \mathrm{~F}\right] \mathrm{EF} 5$ imaging was also shown in a rat model where tumors with high hypoxic values demonstrated a TMR between 1.2-1.7 while for low hypoxic tumors a TMR below unity was observed. ${ }^{41}$ In the tumor models H460, HCT116 and PC3, PET imaging of $\left[{ }^{18} \mathrm{~F}\right] \mathrm{EF} 5$ was performed followed by autoradiography and staining for immunofluorescence hypoxia marker EF5. The spatial relationship was found to be dependent on the tumor model being the highest in the PC3 model followed by the HCT116 and the $\mathrm{H} 460$ model. Notably the uptake of $\left[{ }^{18} \mathrm{~F}\right] \mathrm{EF} 5$ was influenced by the simultaneous injection of nonradioactive EF5 which might have important clinical implication when hypoxia tracer is combined with hypoxia targeting drugs with similar properties. ${ }^{42}$ Furthermore, Silén et al. reported in preclinical head and neck xenograft models that high $\left[{ }^{18} \mathrm{~F}\right] \mathrm{EF} 5$ PET uptake was observed in tumor models having high CAIX, GLUT1 and HIF1 $\alpha$ expression, while low values of these endogenous markers were detected in models with low $\left[{ }^{18} \mathrm{~F}\right] \mathrm{EF5}$ uptake. ${ }^{43}$ 
A first validation study for $\left[{ }^{18} \mathrm{~F}\right] \mathrm{EF} 3$ revealed a biodistribution based TBR ranging from 1.2 to 2.9 and ranking of the different tumor models based on $\left[{ }^{18} \mathrm{~F}\right] \mathrm{EF} 3$ uptake was comparable with ranking based on the amount of hypoxia as detected by EF5 immunofluorescence. ${ }^{44}$ Furthermore a significant correlation was observed between $\left[{ }^{18} \mathrm{~F}\right] \mathrm{EF} 3$ and EF5 immunofluorescence after exposing the tumor bearing animals to either carbogen or $10 \%$ oxygen breathing ${ }^{45}$ while pharmacological intervention could not improve TMR. ${ }^{46}$

\section{$\left[{ }^{18} \mathrm{~F}\right] \mathrm{HX} 4$}

The first validation of $\left[{ }^{18} \mathrm{~F}\right] \mathrm{HX} 4$ was performed in a rat rhabdomyosarcoma model and demonstrated a significant spatial relationship at tumor-regional level between $\left[{ }^{18} \mathrm{~F}\right] \mathrm{HX} 4$ distribution and pimonidazole staining indicating that $\left[{ }^{18} \mathrm{~F}\right] \mathrm{HX} 4$ does accumulate specifically in hypoxic regions. ${ }^{47}$ Furthermore $\left[{ }^{18} \mathrm{~F}\right] \mathrm{HX} 4$ uptake was causally related to the tumor oxygenation, since exposing animals to altered oxygen concentrations resulted in a dependent $\left[{ }^{18} \mathrm{~F}\right] \mathrm{HX} 4$ accumulation in the tumor. Carlin et al. reported that the tracer distribution on autoradiography showed a clear positive relationship with pimonidazole and CAIX staining, indicating that HX4 accumulates in the hypoxic regions. ${ }^{35}$

All PET tracers discussed here show feasibility of detecting hypoxic tumor regions although each tracer has its own characteristics. Since studies are performed in different animal tumor models, using various conditions and validation methods, comparisons between different tracers is difficult. For some tracers this is assessed in separate quantification studies (vide infra).

Overall, from the clinical validation studies we can conclude that there is limited evidence linking hypoxia PET imaging with actual oxygen pressure measurements. This might be due to the limitation of oxygen electrode measurements or biopsies to quantify the heterogeneous presence of hypoxia in 3D, which is assessed with hypoxia PET, or hand the limitation of hypoxia PET to visualize the presence of tumor hypoxia on a micrometer scale given the spatial resolution of current PET CT imaging. Although the uptake of several hypoxia PET tracers correlated with pimonidazole staining in preclinical studies, the use of exogenous pimonidazole hypoxia marker is not very common in clinical studies. Therefore endogenous markers are used which report diverse and inconsistent correlations within or between different tumor types. This diversity might at least partially be explained by possible oxygen-independent regulation of these proteins. Although the often used endogenous hypoxia markers HIF1 $\alpha$, CAIX and GLUT1 are regulated in response to low oxygen concentrations, their expression does not always correlate with the hypoxic regions. ${ }^{48}$ Therefore, the results of comparisons between hypoxia tracers and endogenous markers should be interpreted with caution.

\section{QUANTIFICATION}

Quantification of the tracers' uptake is most often determined on the combined PET/CT images, providing both functional (hypoxia) and anatomical information. The amount of 
tracer present in the tumor can be quantified as the "standardized uptake value" (SUV) or tumor-to-background ratio. Based on predefined thresholds, the hypoxic volume or hypoxic fraction can be calculated. Reproducibility is another important measure in hypoxia PET imaging. Quantification of the hypoxic fraction has no use if data are not reproducible over a short or even longer period. Therefore both quantification and reproducibility are evaluated.

Comparative studies in preclinical tumor models can easily quantify the uptake of multiple tracers within one tumor model hereby providing important information on the performance of the tracers with regard to each other (Figure 2.2). Furthermore the implementation of experimental procedures to increase or decrease the hypoxic fraction can easily be applied to investigate the tracer's behavior to these altered conditions.

A first comparison study investigating both $\left[{ }^{18} \mathrm{~F}\right] \mathrm{FMISO}$ with $\left[{ }^{18} \mathrm{~F}\right] \mathrm{FAZA}$ PET uptake in the Walker 256 rat carcinosarcoma model showed an increase in SUV and TBR values from 1 to 3 hours p.i. The $\left[{ }^{18} \mathrm{~F}\right] \mathrm{FMISO}$ uptake values were higher than those for $\left[{ }^{18} \mathrm{~F}\right] \mathrm{FAZA} .{ }^{49}$ Contradicting results were obtained in EMT6, AR42J and A431 tumor models where biodistribution based TMR was significantly higher for $\left[{ }^{18} \mathrm{~F}\right] \mathrm{FAZA}$ as compared with $\left[{ }^{18} \mathrm{~F}\right] \mathrm{FMISO} .{ }^{50}$ The presence of hypoxia was confirmed using invasive electrode $\mathrm{pO}_{2}$ measurements. Furthermore, exposing animals to $100 \% \mathrm{O}_{2}$ breathing conditions revealed reduced $\left[{ }^{18} \mathrm{~F}\right]$ FAZA PET uptake compared to ambient air breathing conditions, which was confirmed by autoradiography. These results were confirmed in another study on A431 tumor bearing mice. ${ }^{51}$ Although the specific uptake in the tumor was higher for $\left[{ }^{18} \mathrm{~F}\right]$ FMISO, the TBR and TMR were significantly higher for $\left[{ }^{18} \mathrm{~F}\right] \mathrm{FAZA}$ on both PET imaging and biodistribution. Sensitivity to increased oxygen concentrations was reported for $\left[{ }^{18} \mathrm{~F}\right]$ FAZA uptake detected by PET and biodistribution. Accumulation of $\left[{ }^{18} \mathrm{~F}\right] \mathrm{FAZA}$ was indicated to be crucial in the first hour post injection. ${ }^{52}$ Exposing tumor bearing animals to enhanced oxygen concentrations either from 1 hour pre injection till 3 hours post injection or only from 2 minutes pre injection till 1 hour post injection gave similar modified results compared to ambient air breathing.

A preclinical comparative study on $\left[{ }^{18} \mathrm{~F}\right] \mathrm{FETNIM}$ and $\left[{ }^{18} \mathrm{~F}\right] \mathrm{FMISO}$ in $\mathrm{C} 3 \mathrm{H}$ mammary carcinoma bearing mice used biodistribution to investigate TBR and TMR under ambient air and carbogen breathing. For both tracers, TBR was decreased under carbogen breathing with higher ratios for $\left[{ }^{18} \mathrm{~F}\right]$ FETNIM $\left(5.8 \pm 2.5\right.$ for air and $2.8 \pm 1.3$ for carbogen) than for $\left[{ }^{18} \mathrm{~F}\right] \mathrm{FMISO}$ (4.3 \pm 2.0 for air and $2.3 \pm 0.5$ for carbogen) whereas TMR was comparable between the two tracers $\left(\left[{ }^{18} \mathrm{~F}\right]\right.$ FETNIM $6.2 \pm 2.1$ and $\left[{ }^{18} \mathrm{~F}\right]$ FMISO $6.4 \pm 3.3$ both in normal air $) .{ }^{53}$

A comparative study investigated the TBR of $\left[{ }^{18} \mathrm{~F}\right] \mathrm{EF} 3$ and $\left[{ }^{18} \mathrm{~F}\right] \mathrm{FMISO}$ in the same rat rhabdomyosarcoma model. At $2 \mathrm{~h}$ p.i. TBR of $\left[{ }^{18} \mathrm{~F}\right] \mathrm{FMISO}$ was significantly higher than $\left[{ }^{18} \mathrm{~F}\right] \mathrm{EF} 3$, but $\left[{ }^{18} \mathrm{~F}\right] \mathrm{EF} 3$ values at $4 \mathrm{~h}$ p.i were comparable to those of $\left[{ }^{18} \mathrm{~F}\right] \mathrm{FMISO}$. This demonstrates that with respect to TBR values $\left[{ }^{18} \mathrm{~F}\right] \mathrm{EF} 3$ is not superior to $\left[{ }^{18} \mathrm{~F}\right] \mathrm{FMISO} .{ }^{54}$ This was partially confirmed in a FSA II tumor model where $\left[{ }^{18} \mathrm{~F}\right] \mathrm{FMISO}$ TMR was significantly higher only at 55 and 330 minutes compared to $\left[{ }^{18} \mathrm{~F}\right] \mathrm{EF} 3$ and not at other time points. Furthermore, $\left[{ }^{18} \mathrm{~F}\right] \mathrm{EF} 3$ TMR remained low under carbogen breathing, while $\left[{ }^{18} \mathrm{~F}\right] \mathrm{FMISO}$ values kept increasing, suggesting that $\left[{ }^{18} \mathrm{~F}\right] \mathrm{EF} 3$ is more sensitive to reduced hypoxia by 
carbogen breathing. ${ }^{55}$ Overall, $\left[{ }^{18} \mathrm{~F}\right] \mathrm{EF} 3$ seems to be a good hypoxia tracer although it might not be superior to $\left[{ }^{18} \mathrm{~F}\right] \mathrm{FMISO}$.

A comparative study performed in our laboratory characterized $\left[{ }^{18} \mathrm{~F}\right] \mathrm{FMISO},\left[{ }^{18} \mathrm{~F}\right] \mathrm{FAZA}$ and $\left[{ }^{18} \mathrm{~F}\right] \mathrm{HX} 4$ within one rhabdomyosarcoma tumor model on TBR, reproducibility and reversibility. ${ }^{56}$ Within this tumor model $\left.{ }^{18} \mathrm{~F}\right] \mathrm{HX} 4$ reached the highest TBR at $3 \mathrm{~h}$ p.i. while $\left[{ }^{18} \mathrm{~F}\right] \mathrm{FAZA}$ reached a stable TBR at $2 \mathrm{~h}$ p.i. and $\left[{ }^{18} \mathrm{~F}\right] \mathrm{FMISO}$ did not reach a plateau. Two PET acquisitions over a 48 hour time period revealed high reproducibility and good overlap in hypoxic regions for both $\left[{ }^{18} \mathrm{~F}\right] \mathrm{FMISO}$ and $\left[{ }^{18} \mathrm{~F}\right] \mathrm{HX} 4$, while $\left[{ }^{18} \mathrm{~F}\right] \mathrm{FAZA}$ did perform worse on this characteristic. Furthermore, $\left[{ }^{18} \mathrm{~F}\right] \mathrm{FMISO}$ showed to be sensitive to decreasing hypoxic fractions while $\left[{ }^{18} \mathrm{~F}\right] \mathrm{FAZA}$ and $\left[{ }^{18} \mathrm{~F}\right] \mathrm{HX} 4$ were responsive to increasing hypoxia. These alterations of oxygen concentrations were introduced by exposing animals to either high or low oxygen concentrations 2.5 hours after tracer injection while PET acquisition was performed just before and immediately after this modification. ${ }^{56}$ In a SQ20b head and neck xenograft model microPET imaging was performed at only one time point, 80-90 minutes p.i., and demonstrated a tumor to muscle ratio of $1.6 \pm 0.4$ for $\left[{ }^{18} \mathrm{~F}\right] \mathrm{FMISO}, 1.4 \pm 0.1$ for $\left[{ }^{18} \mathrm{~F}\right] \mathrm{HX} 4$ and $1.3 \pm 0.3$ for $\left[{ }^{18} \mathrm{~F}\right] \mathrm{FAZA} .{ }^{35}$

The previously described tracers $\left[{ }^{18} \mathrm{~F}\right] \mathrm{FMISO},\left[{ }^{18} \mathrm{~F}\right] \mathrm{FAZA},\left[{ }^{18} \mathrm{~F}\right] \mathrm{HX} 4,\left[{ }^{18} \mathrm{~F}\right] \mathrm{EF}$, and $\left[{ }^{18} \mathrm{~F}\right] \mathrm{FETNIM}$ were used for hypoxia PET imaging in patients with head and neck cancer (Figure 2.2; $\left[{ }^{18} \mathrm{~F}\right] \mathrm{HX} 4$ image of head and neck cancer patient). A huge variation of data is reported with no standardized imaging acquisition protocol or image analysis. The PET imaging time point p.i. ranges from $1.5 \mathrm{~h}$ to $4 \mathrm{~h}$ p.i. In addition, the selected background tissue ("normal" tissue, muscle, blood, cerebellum) and the thresholds to define tumor hypoxia are diverse; for example, selecting the voxels with a tumor-to-background $\geq 1.2, \geq 1.25, \geq 1.3, \geq 1.4, \geq 1.5$, or selecting voxels above the background uptake +3 standard deviations. This makes it very difficult or even impossible to compare the different tracers based on the currently available clinical literature. Only one study compared two tracers in the same patient population; however the authors used different acquisition protocols. ${ }^{57}\left[{ }^{18} \mathrm{~F}\right] \mathrm{FMISO}$ imaging was performed at $2 \mathrm{~h}$ p.i., while $\left[{ }^{18} \mathrm{~F}\right] \mathrm{HX} 4$ imaging was performed at $1.5 \mathrm{~h}$ p.i. The study showed similar hot spots for both tracers and comparable TBR ratios for the time points used.

In patients with NSCLC the tracers $\left[{ }^{18} \mathrm{~F}\right] \mathrm{FMISO},\left[{ }^{18} \mathrm{~F}\right] \mathrm{FAZA}$ and $\left[{ }^{18} \mathrm{~F}\right] \mathrm{HX} 4$ were used to visualize tumor hypoxia (Figure 2.2; $\left[{ }^{18} \mathrm{~F}\right] \mathrm{HX} 4$ image of lung cancer patient). Rasey et al. already quantified tumor hypoxia, using $\left[{ }^{18} \mathrm{~F}\right] \mathrm{FMISO}$, in 1996 . They observed tumor hypoxia in all NSCLC lesions, however a broad range of the extent of hypoxia was shown with a fractional hypoxic volume ranging from 1.3-94.7\%. ${ }^{2}\left[{ }^{18} \mathrm{~F}\right] \mathrm{FAZA}$ PET/CT imaging in patients with NSCLC was performed by Postema et $a l^{58}$ and Trinkaus et $a .^{59}$ who observed tumor hypoxia in respectively $54 \%$ and $65 \%$ of the patients. These results were confirmed with $\left[{ }^{18} \mathrm{~F}\right] \mathrm{HX} 4$, reporting an increased uptake in $72 \%$ of all NSCLC lesions. ${ }^{60}$ All these results show that the majority of NSCLC lesions are hypoxic and therefore lung cancer patients could be good candidates for hypoxia targeted therapies. 


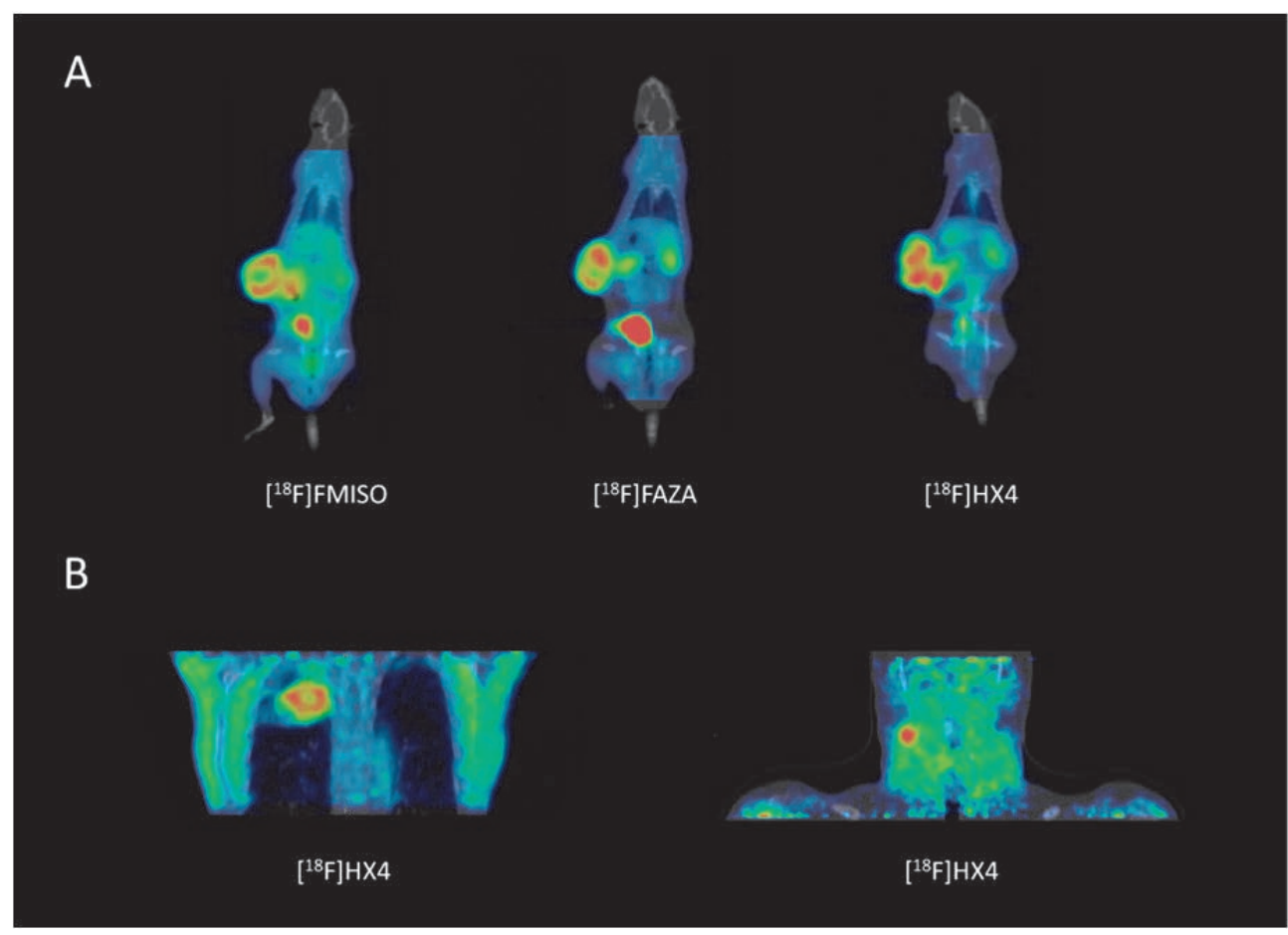

Figure 2.2: A) preclinical PET/CT images $\left[{ }^{18} \mathrm{~F}\right] \mathrm{FMISO},\left[{ }^{18} \mathrm{~F}\right] \mathrm{FAZA}$ and $\left[{ }^{18} \mathrm{~F}\right] \mathrm{HX} 4$ uptake in a rhabdomyosarcoma bearing rat at 4 hours post injection. B) the clinical images visualize a lung (left) and a Head and Neck (right) cancer patient at 4 hours post injection using $\left.{ }^{18} \mathrm{~F}\right] \mathrm{HX} 4 \mathrm{PET} / \mathrm{CT}$ imaging. Color scale ranges from blue (low uptake) to red (high uptake).

$\left[{ }^{18} \mathrm{~F}\right] \mathrm{FMISO}$ was also used to quantify tumor hypoxia in patients with brain cancer, pancreatic cancer and renal cell carcinoma. In 11 patients with glioblastoma an average tumorto-blood ratio of $1.5 \pm 0.2$ was reported. ${ }^{61}$ This $\left[{ }^{18} \mathrm{~F}\right.$ FMISO TBR was significantly correlated to the biological aggressiveness measured by tumor growth kinetics on MRI. In addition it was observed that the $\left[{ }^{18} \mathrm{~F}\right] \mathrm{FMISO}$ uptake (tumor to cerebellum ratio) was significantly higher for the patients with glioblastoma $(2.7 \pm 0.6)$, in comparison to the other glioma patients (1.2 \pm 0.1$).{ }^{62}$ These results were confirmed by Yamamoto et al., who observed no hypoxia (TBR $<1.2$ ) in the grade I and II gliomas, and a significantly increasing TBR for grade III $(1.8 \pm 0.6)$ and IV $(2.5 \pm 0.4) .{ }^{63}$ Therefore, in this patient population, the quantification of tumor hypoxia could contribute to the staging of patients with glioma.

In patients with pancreatic carcinoma, a visual increased uptake of $\left[{ }^{18} \mathrm{~F}\right] \mathrm{FMISO}$ was only observed in 2 out of 10 patients, however all patients showed a $T B R \geq 1.4$, indicating uptake in hypoxic regions. Based on the difficulty to visually assess tumor hypoxia in patients with pancreatic cancer, hypoxia PET imaging should be combined with other modalities for lesion localization. ${ }^{64}$ The $\left[{ }^{18} \mathrm{~F}\right] \mathrm{FMISO}$ uptake in 11 patients with renal cell carcinoma was mildly increased, with an average tumor SUVmax of $1.3 \pm 0.3$ in comparison to the normal kidney tissue $1.1 \pm 0.2^{30}$ 
In patients with rectal cancer both $\left[{ }^{18} \mathrm{~F}\right] \mathrm{FMISO}$ and $\left[{ }^{18} \mathrm{~F}\right] \mathrm{FAZA}$ was used to assess tumor hypoxia. Loi et al performed $\left[{ }^{18} \mathrm{~F}\right] \mathrm{FMISO}$ PET imaging, as part of a phase 1 trial in 6 patients with rectal cancer. Tumor hypoxia was observed in $67 \%$ of the primary lesions and in $83 \%$ of the metastases at baseline. ${ }^{65}$ In addition it was reported that $\left[{ }^{18} \mathrm{~F}\right] \mathrm{FAZA}$ uptake in rectal tumors was significantly higher than the reference tissues, with an average tumor to muscle ratio of $2.0 .^{66}$ An additional, important message in this manuscript is a practical issue regarding the influence of scattered activity from the bladder, which can influence the quantitative analysis of $\left[{ }^{18} \mathrm{~F}\right]$ FAZA PET uptake in the rectum. $\left[{ }^{18} \mathrm{~F}\right] \mathrm{FAZA}$ was additionally used to investigate tumor hypoxia in patients with prostate carcinoma, with negative results. The attempt to visualize tumor hypoxia in 14 patients with histology proven prostate cancer failed to show any significant uptake in the tumor lesions. ${ }^{67}\left[{ }^{18}\right.$ F]FETNIM hypoxia PET imaging was used to describe tumor hypoxia in 28 patients with esophageal carcinoma. Increased uptake of $\left[{ }^{18}\right.$ F]FETNIM inside the primary tumor was observed which was the first evidence for hypoxia PET quantification in esophageal cancer.

From all these studies, it can be concluded that the imaging contrast of nitroimidazole based hypoxia tracers is limited, however sufficient to quantify tumor hypoxia and extract valuable information for most clinical tumor types.

\section{PHARMACOKINETIC ANALYSIS}

Besides static PET imaging, quantification of tumor hypoxia can be performed using dynamic PET acquisitions and image analysis. This pharmacokinetic analysis can be used to provide additional tumor specific values of tracer retention and tumor perfusion. In a preclinical investigation in Dunning R3327-AT prostate tumor bearing nude rats the relationship between the $\left[{ }^{18} \mathrm{~F}\right] \mathrm{FMISO}$ pharmacokinetic rate constants was assessed, using an irreversible two-tissue compartment model, in relationship to pO2 measurements. They showed that the rate-constant $k 3$, representing the trapping rate, was better than the standard tumor-to-plasma ratio, to identify tumor hypoxia. ${ }^{68}$ In addition Busk et al. performed pharmacokinetic modeling of $\left[{ }^{18} \mathrm{~F}\right] \mathrm{FAZA}$ in three murine squamous cell carcinomas using also an irreversible two-tissue compartment model. The time activity curves of the tumors varied between the different tumor types. Correlation of the rate-constants to static 3 h p.i. [ ${ }^{18}$ F]FAZA PET imaging, showed a poor correlation for k3 for 2 out of 3 tumor types, however a good correlation was observed with $\mathrm{Ki}$, the influx rate constant; $(k 1 \times k 3) /(k 2+k 3) .{ }^{69}$ Clinically pharmacokinetic analysis of $\left[{ }^{18} \mathrm{~F}\right] \mathrm{FMISO}$ was only performed in patients with head and neck cancer. In agreement with pre-clinical data, an irreversible 2tissue compartment model was used to describe the kinetics of $\left[{ }^{18} \mathrm{~F}\right] \mathrm{FMISO} .{ }^{70,71} \mathrm{~A}$ positive correlation was observed between the influx rate $\mathrm{Ki}$ and the standard tumor to blood ratio, measured on static $\left[{ }^{18} \mathrm{~F}\right] \mathrm{FMISO}$ images. ${ }^{70}$ Another study used pharmacokinetic modeling to differentiate between tumor perfusion and hypoxia, providing the opportunity to stratify patients in three categories, 1) high perfusion, no hypoxia; 2) perfusion and hypoxia; and 3) severe hypoxia and reduced perfusion, which could be related to the radiotherapy outcome. For $\left[{ }^{18} \mathrm{~F}\right] \mathrm{FAZA}$, kinetic analysis was clinically performed in patients with NSCLC. Three different compartment models were evaluated; the reversible single- 
tissue, reversible two-tissue and irreversible two-tissue compartment model. In this patient population, the reversible two-tissue compartment model is found to best represent $\left[^{18} \mathrm{~F}\right]$ FAZA kinetics. ${ }^{72}$ Using this model, the calculated volume of distribution $\left(\mathrm{V}_{\mathrm{T}} ; \mathrm{k} 1 / \mathrm{k} 2\right.$ $(1+k 3 / k 4))$ showed a good correlation to the statically measured SUV values and TBR. From the clinical literature we observe a difference in model preference for $\left[{ }^{18} \mathrm{~F}\right] \mathrm{FMISO}$, irreversible and $\left[{ }^{18} \mathrm{~F}\right] \mathrm{FAZA}$, reversible, which could be explained by the different properties of the tracers. However, studies were performed in different patient populations (head and neck versus NSCLC). To make a useful comparison kinetic analysis of the different tracers within the same patient population is necessary. Note that for both $\left[{ }^{18} \mathrm{~F}\right] \mathrm{FMISO}$ and $\left[{ }^{18} \mathrm{~F}\right] \mathrm{FAZA}$, the kinetic parameters $\mathrm{Ki}$ or $\mathrm{V}_{\mathrm{T}}$ were correlated to the static parameter TBR or SUV, which justifies a standard static analysis for these PET tracers.

\section{APPLICATIONS}

PET hypoxia imaging can be used for multiple applications. It has been shown to have a prognostic value and can be useful in the monitoring of tumor hypoxia during the course of treatment. Furthermore the assessment of tumor hypoxia can be used to select patients that might benefit from a certain therapy.

\section{Prognostic/ predictive value of hypoxia PET imaging}

In a preclinical setting the prognostic value of $\left[{ }^{18} \mathrm{~F}\right] \mathrm{FAZA}$ was assessed by performing a hypoxia PET scan combined with the invasive oxygen sensitive electrode measurement, followed by a single dose of irradiation (55Gy). Most animals were exposed to ambient oxygen breathing while a fraction of the animals was exposed to carbogen breathing from 10 minutes prior to and during both hypoxia measurements and radiotherapy. Both methods could distinguish between the more and less hypoxic tumors resulting in significantly higher local tumor control. ${ }^{73}$ Another study reported that discrimination between normoxic and hypoxic tumors based on pretreatment $\left[{ }^{18} \mathrm{~F}\right]$ FAZA PET imaging could predict the effectiveness of the combined treatment of radiotherapy with the hypoxia specific cytotoxin tirapazamine. $^{74}$

In the clinic the relationship between hypoxia PET imaging and treatment outcome was investigated by several groups (Table 2.I). The use of $\left[{ }^{18} \mathrm{~F}\right] \mathrm{FMISO},\left[{ }^{18} \mathrm{~F}\right] \mathrm{FETNIM}$ and $\left[{ }^{18} \mathrm{~F}\right] \mathrm{EF5}$ was correlated with the outcome of head and neck cancer patients. Lehtio et al. showed in 21 patients that the fractional hypoxic volume, measured before treatment on $\left[{ }^{18}\right.$ F]FETNIM PET imaging, was predictive for 3 -years overall survival. ${ }^{75}$ In addition it was reported that there is a significant decrease in disease free survival for hypoxic versus non-hypoxic tumors. ${ }^{76,77}$ On the other hand, no correlation was shown between outcome and pre- or mid-treatment $\left[{ }^{18} \mathrm{~F}\right] \mathrm{FMISO}$ imaging for patients treated with several cycles of platinum based chemotherapy and IMRT (Intensity Modulated Radiation Therapy). However, this conclusion was based on a 3-year local and regional progression-free survival rate of $100 \%$ and $95 \%$. Only one patient showed regional failure after treatment, but did not have a positive $\left[{ }^{18} \mathrm{~F}\right] \mathrm{FMISO}$ PET/CT scan during treatment. ${ }^{78}$ The association between 
local progression-free survival and $\left[{ }^{18} \mathrm{~F}\right] \mathrm{FMISO}$ PET imaging before and during chemoradiotherapy was investigated and showed that $\left[{ }^{18} \mathrm{~F}\right] \mathrm{FMISO}$ PET imaging during treatment had a higher prognostic power than baseline hypoxia imaging. ${ }^{79}$ In addition, parameters extracted from the kinetic analysis of $\left[{ }^{18} \mathrm{~F}\right] \mathrm{FMISO}$ PET imaging and its changes during treatment were highly correlated to radiotherapy outcome. ${ }^{80,81}$

Rischin et al. for the first time demonstrated the predictive value of $\left[{ }^{18} \mathrm{~F}\right] \mathrm{FMISO}$ in patients with head and neck cancer randomized in a Phase II trial for chemoradiotherapy alone or in combination with hypoxic cell cytotoxin tirapazamine. Pretreatment $\left[{ }^{18} \mathrm{~F}\right] \mathrm{FMISO}$ tumor hypoxia was associated with a high risk of locoregional failure after chemoradiotherapy alone. A considerable improvement in locoregional control was found in patients with hypoxic tumors treated with tirapazamine, whereas tirapazamine could not improve the outcome of chemoradiotherapy in non-hypoxic tumors. ${ }^{82}$

Several studies investigated the prognostic value of hypoxia PET imaging for patients with head and neck cancer in comparison to standard metabolic PET imaging using $\left[{ }^{18} \mathrm{~F}\right] \mathrm{FDG}$. In an univariate analysis it was shown that the nodal stage and $\left[{ }^{18} \mathrm{~F}\right] \mathrm{FMISO}$ PET parameters (TBR and hypoxic volume) were significantly correlated with survival, while the $\left[{ }^{18} \mathrm{~F}\right] \mathrm{FDG}$ SUV $_{\max }$ was not. $^{83}$ These results were confirmed by another study which observed a significant correlation between FMISO PET uptake and chemotherapy response, while $\left[{ }^{18}\right.$ F]FDG uptake was not correlated. ${ }^{26} \mathrm{~A}$ relationship between overall survival, the hypoxic subvolume, defined on $\left[{ }^{18} \mathrm{~F}\right] \mathrm{EF} 5$ PET imaging, and the metabolic active tumor volume, defined on the $\left[{ }^{18} \mathrm{~F}\right]$ FDG-PET was observed. ${ }^{84}$ However, also in this study, the hypoxia PET uptake was stronger correlated with overall survival in comparison to $\left[{ }^{18} \mathrm{~F}\right] \mathrm{FDG}$. All these studies show the prognostic potential of hypoxia PET imaging over metabolic $\left[{ }^{18}\right.$ F]FDG PET imaging. However, Thorwarth et al. showed in a small cohort of patients $(\mathrm{N} .=12)$ that a parameter based on the voxel-wise comparison of both tracers quantifying discrepancies between $\left[{ }^{18} \mathrm{~F}\right] \mathrm{FMISO}$ and $\left[{ }^{18} \mathrm{~F}\right] \mathrm{FDG}$ PET was the most significant variable, ${ }^{85}$ suggesting that a combination of two tracers on a voxel level may increase both their prognostic significance. 


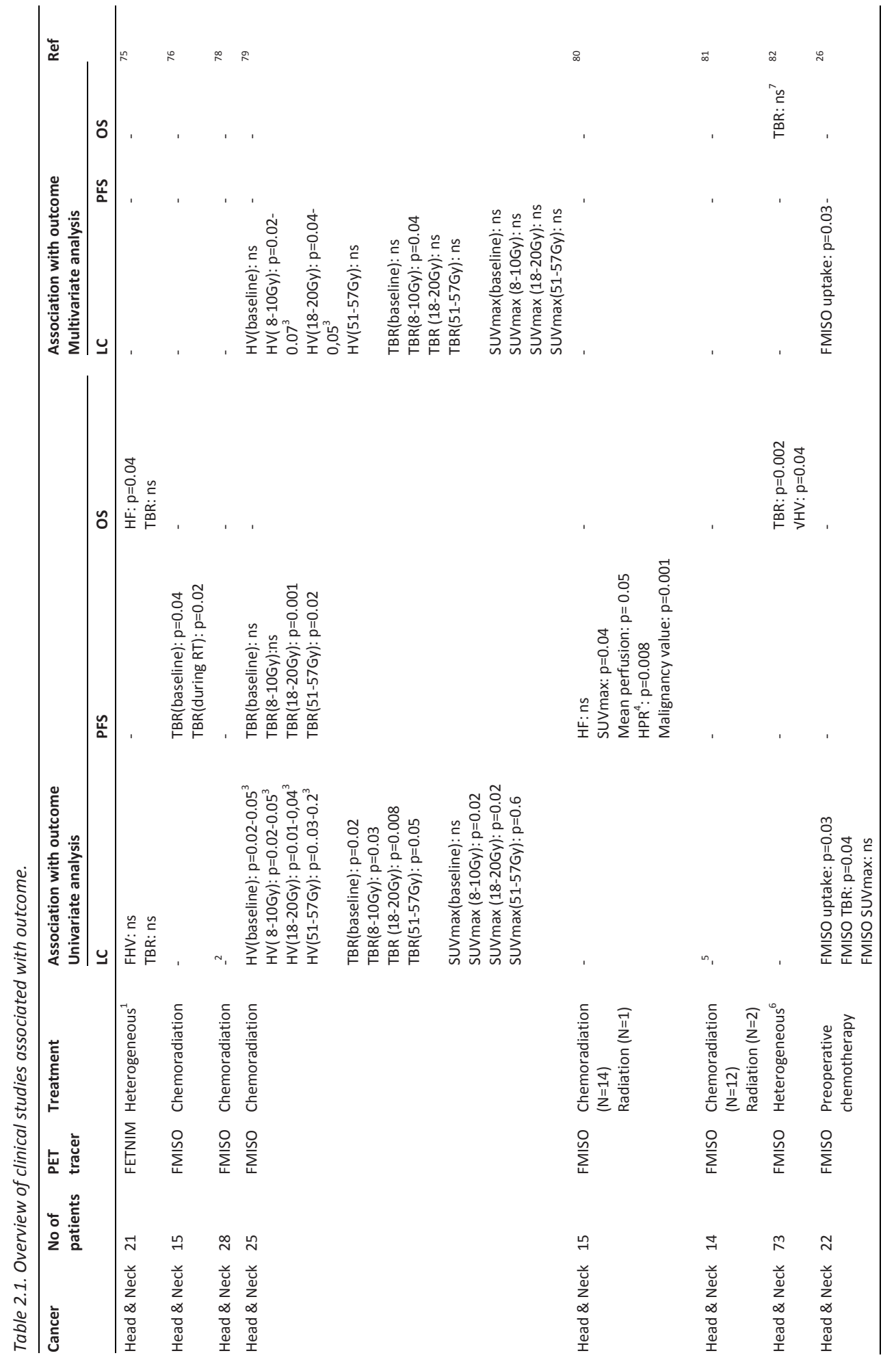




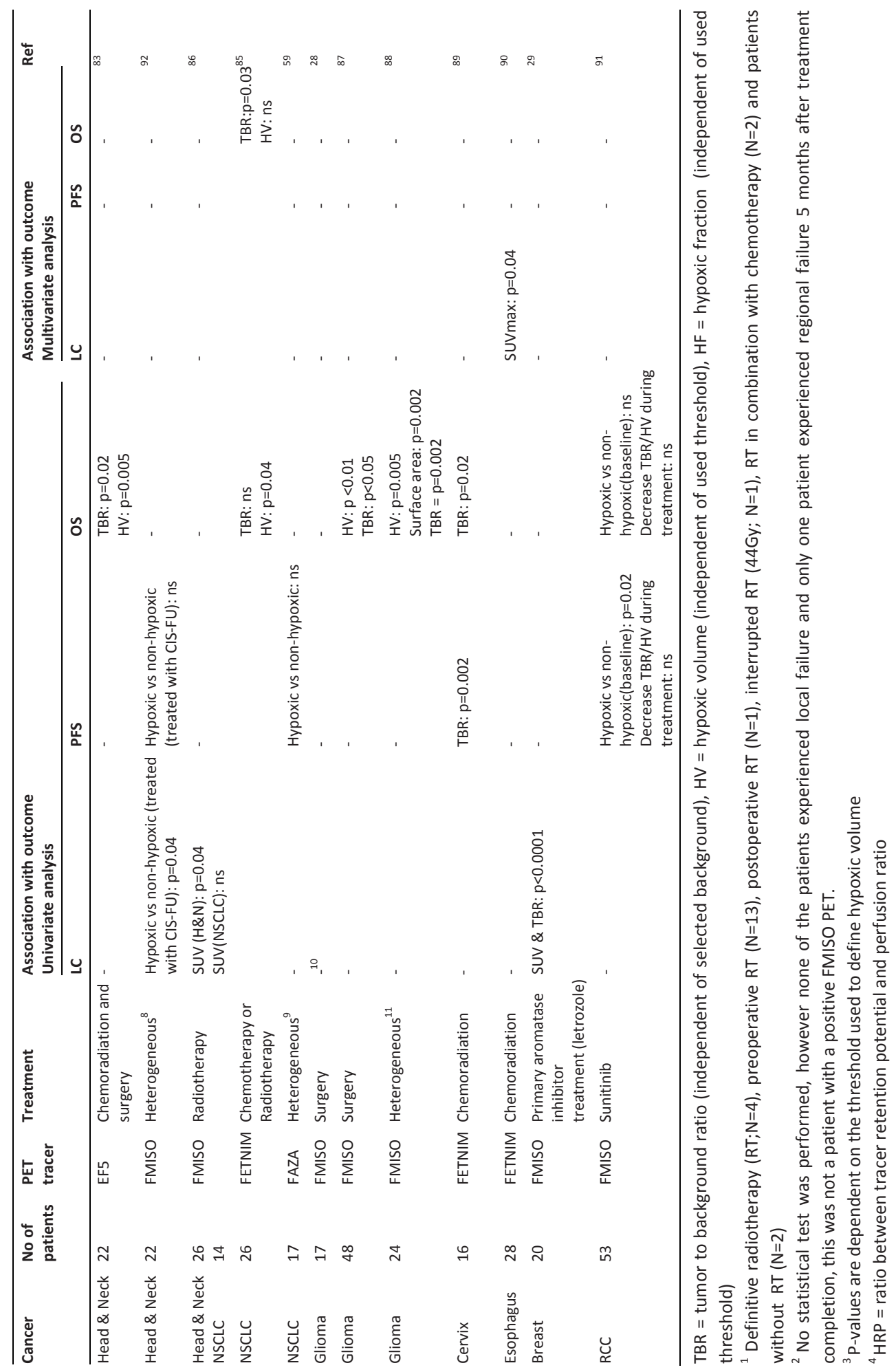




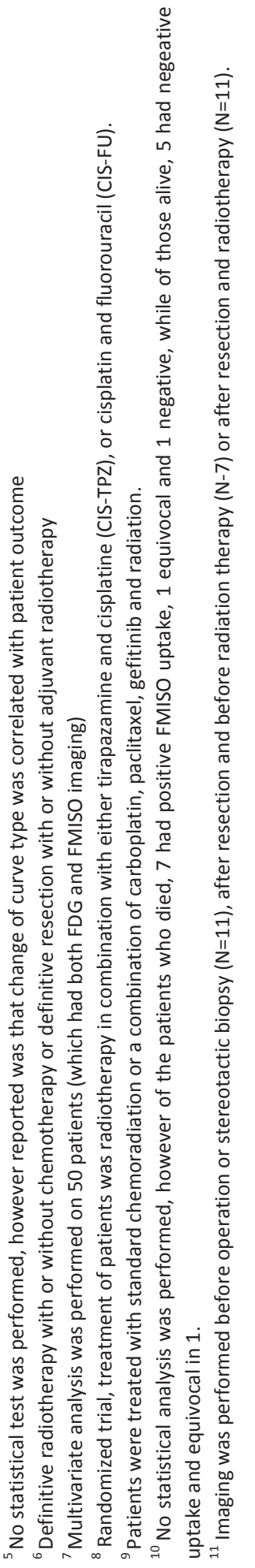


Hypoxia PET imaging was also used to predict the outcome of patients with lung cancer. It was observed that the TBR and hypoxic volume defined on $\left[{ }^{18}\right.$ F]FETNIM PET were predictors of overall survival in a univariate analysis, while the $\left[{ }^{18} \mathrm{~F}\right] \mathrm{FDG}$ uptake was not significant. ${ }^{86}$ The $\left[{ }^{18} \mathrm{~F}\right]$ FMISO PET time activity curve type (i.e. rapid washout, delayed washout or accumulation) and a tumor to muscle ratio of 2.0 were shown to be good parameters to predict tumor response. ${ }^{87}$ On the other hand no significant difference was observed in disease-free survival between NSCLC patients $(\mathrm{N}=17)$ having a hypoxic or a non-hypoxic lesions. ${ }^{59}$ For patients with glioma, $\left[{ }^{18} \mathrm{~F}\right] \mathrm{FMISO}$ PET imaging was observed to be prognostic for treatment outcome. ${ }^{28}$ The majority of patients who died of progressive disease had positive $\left[{ }^{18} \mathrm{~F}\right] \mathrm{FMISO}$ uptake, while for those that were still alive, $\left[{ }^{18} \mathrm{~F}\right] \mathrm{FMISO}$ was negative in most cases. In addition, an increased TBR or hypoxic volume defined on $\left[{ }^{18} \mathrm{~F}\right] \mathrm{FMISO}$ PET imaging was found to be significantly related to a decreased overall survival in patients with newly diagnosed glioma. ${ }^{88,89}$

$\left[{ }^{18} \mathrm{~F}\right]$ FETNIM uptake was significantly correlated with progression-free survival in patients with cervical carcinoma. ${ }^{90}$ In addition, the $\left[{ }^{18}\right.$ F]FETNIM SUV ${ }_{\max }$ was the best predictor of treatment response in comparison to tumor length, diameter, and SUV $\mathrm{V}_{\text {mean }}$ in patients with esophageal carcinoma. ${ }^{91}$ Cheng et al. evaluated $\left[{ }^{18} \mathrm{~F}\right.$ FMISO PET imaging in patients with breast cancer for the prediction of treatment resistance to hormonal therapy. ${ }^{29}$ They observed a significant correlation between baseline $\left[{ }^{18} \mathrm{~F}\right] \mathrm{FMISO}$ uptake and clinical outcome. Using a TBR threshold $\geq 1.2$ to quantify tumor hypoxia allowed correct prediction for $88 \%$ of the patients with progressive disease. Comparison of $\left[{ }^{18} \mathrm{~F}\right] \mathrm{FMISO}$ PET imaging at baseline and follow-up showed that the patients with a partial response or stable disease showed a decreasing trend of the $\left[{ }^{18} \mathrm{~F}\right] \mathrm{FMISO}$ TBR, while patients with progressive disease did not. In patients with renal cell carcinoma, the presence of baseline tumor hypoxia was related to a shorter progression-free survival, however overall survival was not related to $\left[{ }^{18} \mathrm{~F}\right] \mathrm{FMISO}$ uptake. Also changes in tumor hypoxia during treatment were not prognostic for progression-free or overall survival. ${ }^{92}$

In the previous paragraph "validation" we have described that there is limiting evidence linking hypoxia PET imaging with actual oxygen pressure measurements in patients. However given the prognostic value of hypoxia PET imaging in various tumor types, the question is how we should approach validation of our current hypoxia PET imaging techniques in clinical practice. Given the highly prognostic value of hypoxia PET imaging, which is in general stronger than the other available (imaging) parameters, it shows that there is added prognostic and maybe predictive value in hypoxia imaging. Therefore, hypoxia PET imaging could be integrated in clinical trials which have the aim to improve loco-regional control or survival, especially for hypoxia modifying treatments.

\section{Monitoring tumor hypoxia during treatment}

Experimental and clinical data demonstrate that tumor hypoxia plays an important role in malignant progression and resistance to anticancer treatments. Hypoxia PET imaging can be used as a non-invasive tool to evaluate the effectiveness of therapy to eliminate hypoxic tumor cells and adapt treatment accordingly. This was studied in some preclinical 
studies giving a first indication about the possibilities. However treatment schedules as they are applied to patients are hard to mimic in preclinical setting.

Acquisition of a $\left[{ }^{18} \mathrm{~F}\right.$ FMISO PET scan was performed before and after the treatment of a HT29 xenograft tumor using the antivascular compound vadimezan. ${ }^{93}$ A reduced $\left[{ }^{18} \mathrm{~F}\right]$ FMISO uptake 24 hours after vadimezan administration was observed only in a part of the treatment group. Quantification of the tumor microvasculature revealed that this was most likely due to changes in perfusion and could probably not be assigned to changes in hypoxia. The antiangiogenic treatment with sunitinib also reduced the $\left[{ }^{18} \mathrm{~F}\right] \mathrm{FMISO}$ uptake after 7 days of treatment in an orthotopic glioma model while the cerebral blood volume (CBV) was increased and the vessel permeability was decreased. ${ }^{94}$ Control animals were unaffected in CBV but had high hypoxic values. However, in a renal cell carcinoma xenograft model, the hypoxic fraction detected by $\left[{ }^{18} \mathrm{~F}\right] \mathrm{FMISO}$ was increased after 2, 3 and 7 days of treatment with Sorafenib, an antiangiogenic drug, compared to control. ${ }^{95}$ This was supported by immunohistochemical data indicating that the mean vessel density decreased, while necrosis in the tumor increased.

In a preclinical cervix tumor model two $\left[{ }^{18} \mathrm{~F}\right]$ FAZA PET scans were acquired on consecutive days before the start of fractionated radiotherapy, followed by a $\left[{ }^{18} \mathrm{~F}\right]$ FAZA PET scan after 4 and 10 fractions of $2.5 \mathrm{~Gy} .{ }^{96}$ Voxel to voxel analysis revealed a good reproducibility on the pretreatment scans, but the overall $\left[{ }^{18} \mathrm{~F}\right] \mathrm{FAZA}$ uptake changed over treatment. Treatment of $\mathrm{A} 431$ bearing animals with the pan-erbB tyrosine kinase inhibitor $\mathrm{Cl}-1033$ was monitored using hypoxia PET imaging pretreatment and on day 3,6 or $7 .^{97}$ Therapy was shown to be effective since shrinkage of the treated tumor occurred. This was reflected in the $\left[{ }^{18} \mathrm{~F}\right]$ FAZA uptake which decreased over treatment from day 0 to day 3 and an even further decrease on day 6 . $\left[{ }^{18} \mathrm{~F}\right] \mathrm{FMISO}$ imaging at day 7 confirmed the low uptake in the $\mathrm{Cl}-1033$ treated animals while the control animals had high tracer uptake for both $\left[{ }^{18} \mathrm{~F}\right]$ FAZA and $\left[{ }^{18} \mathrm{~F}\right] \mathrm{FMISO}$. The efficacy of another tyrosine kinase inhibitor (Gefitinib) was tested in the same tumor model showing similar results. ${ }^{98}$ The amount of hypoxia in the treatment group is reduced as measured by $\left[{ }^{18} \mathrm{~F}\right]$ FAZA PET imaging, while the control group remained hypoxic. $\left[{ }^{18} \mathrm{~F}\right] \mathrm{EF} 5$ PET imaging was used to monitor treatment response of a bioreductive drug combined with radiotherapy. ${ }^{99}$ This showed a reduction in SUV comparing $24 \mathrm{~h}$ post treatment with a pretreatment scan indicative for tumor response.

These preclinical studies show a discrepancy in hypoxia PET imaging after the use of antivascular treatments which is possibly caused by differences in perfusion as a result of the treatment. The monitoring of other anticancer therapies did show consistent results, indicating that hypoxia PET imaging is suitable for this kind of studies.

Several clinical studies focused on the monitoring of hypoxia using non-invasive PET imaging during treatment. In patients with head and neck squamous cell carcinoma a decreased uptake of $\left[{ }^{18} \mathrm{~F}\right] \mathrm{FMISO}$ after treatment with neoadjuvant chemotherapy was reported. ${ }^{100}$ In addition, in $89 \%$ of the patients $\left[{ }^{18} \mathrm{~F}\right] \mathrm{FMISO}$ uptake was completely absent after treatment with one cycle of chemotherapy (5-fluororacil \& carboplatin) and 40Gy of IMRT. ${ }^{78}$ Servagi-Vernat reported the uptake of $\left[{ }^{18}\right.$ F]FAZA before and during concomitant chemo-radiotherapy in the primary tumor and the involved lymph nodes. In all lesions, the 
fractional hypoxic volume and SUV ${ }_{\max }$ decreased after 17 fractions of radiotherapy. ${ }^{101}$ The changes in $\left[{ }^{18} \mathrm{~F}\right] \mathrm{FMISO}$ kinetics after treatment with (chemo-)radiotherapy were also studied. Three different curve types were defined, representing a severe, intermediate and low degree of hypoxia. ${ }^{80}$ Based on these curves, a decreased amount of hypoxia in the majority of patients was shown. From these studies we can conclude that in patients with head and neck cancer, hypoxia decreases during (chemo-) radiation treatment, indicating therapy induced reoxygenation.

In patients with NSCLC, the evaluation of hypoxia changes during treatment was investigated using $\left[{ }^{18} \mathrm{~F}\right]$ FMISO PET imaging. One study observed an average decrease in tumor hypoxia during radiotherapy, however the changes were unpredictable and only one patient had no detectable tumor hypoxia by the end of radiotherapy. ${ }^{102}$ Another study observed no significant changes in $\left[{ }^{18} \mathrm{~F}\right] \mathrm{FMISO}$ uptake before and during treatment with chemo-radiotherapy. ${ }^{103}$ On the other hand in a study using $\left[{ }^{18} \mathrm{~F}\right] \mathrm{FAZA}$, tumor hypoxia was eliminated after treatment with chemo-radiotherapy in the majority of NSCLC patients. ${ }^{59}$ For cervical carcinoma $\left[{ }^{18} \mathrm{~F}\right] \mathrm{FAZA}$ PET imaging was performed before, during and after radiotherapy. Before the start of radiotherapy positive $\left[{ }^{18} \mathrm{~F}\right] \mathrm{FAZA}$ uptake was observed in 5 out of 15 patients. During treatment persistent hypoxia was observed in 4 patients, while after radiotherapy no remaining hypoxia was observed. ${ }^{104}$ As part of a multimodality imaging trial $\left[{ }^{18} \mathrm{~F}\right] \mathrm{FMISO}$ imaging was performed in sarcomas before, during and after chemotherapy. ${ }^{105}$ All soft tissue sarcomas presented a hypoxic volume before the start of treatment, which remained present during and after treatment. The changes of tumor hypoxia during treatment were diverse among the patient population. Rajendran et al. confirms these results, reporting tumor hypoxia in 14 out of 19 patients with soft tissue sarcoma, which decreased, increased or remained stable during treatment in 6, 2 and 2 patients respectively. ${ }^{106}$ Both studies reported that there was no association between the hypoxic volume in soft tissue sarcomas and the tumor grade.

Comparing the results of the different tumor types, it is striking that in majority of head and neck studies a decrease of tumor hypoxia is observed during treatment, while these results are diverse in NSCLC, cervical cancer and sarcomas. This might suggest that tumor hypoxia is more persistent in these cancer types, or the given treatment is not effective in eradicating the hypoxic cells. This might also explain the results of Overgaard et al. showing that oxygen modification in addition to radiotherapy provides a significantly increased tumor control for head and neck cancer patients, however not for uterine cervix or NSCLC. ${ }^{107}$

\section{Hypoxia targeting}

Based on clinical studies showing an association between hypoxia and treatment outcome, hypoxia PET imaging might be a powerful tool for patient selection and treatment adaptation. The available literature mainly focuses on the adaptation of radiotherapy based on hypoxia PET imaging information and so far only feasibility studies have been reported. Furthermore the patients that are sensitive to hypoxia targeted treatments could be selected, moving in the direction of individualized medicine. 
One possibility to target hypoxic tumor subvolumes is to escalate radiation dose. The feasibility of $\left[{ }^{18} \mathrm{~F}\right]$ FMISO PET based IMRT to the hypoxic tumor volume was investigated for 8 patients with head and neck cancer. ${ }^{108}$ They were able to perform a dose-escalation (78Gy) to the hypoxic tumor volume in 6 out of 8 patients without increasing the normal tissue doses. Additionally, $\left[{ }^{18} \mathrm{~F}\right] \mathrm{FMISO}$-based boost plans could provide an increased tumor control probability without increasing expected complications. ${ }^{109} \mathrm{~A}$ theoretical model also indicated the potential for dose sparing by focusing the dose to the radioresistant hypoxic tumor volumes. ${ }^{110}$ In addition, Chang et al. performed a biological modeling study in 8 HNSCC patients, using $\left[{ }^{18}\right.$ F]FMISO PET imaging for radiotherapy dose painting. ${ }^{111}$ They compared the standard radiotherapy plan with uniform dose escalation and hypoxiabased dose painting. Hypoxia-based dose painting demonstrated the advantage to increase the tumor control probability without increasing the normal tissue toxicity, therefore increasing the chances for an uncomplicated tumor control probability. These results confirm that it is theoretically possible to perform hypoxia-based radiotherapy in patients with head and neck cancer. However, it is essential to know whether the defined hypoxic volume is accurate and extensive enough to target the radioresistant areas. For example, it was shown that all recurrences $(\mathrm{N}=9)$ after chemoradiotherapy were located within the $\left[{ }^{18} \mathrm{~F}\right]$ FDG avid regions, however 3 of these recurrences were located outside the baseline hypoxic volume, defined on $\left[{ }^{18}\right.$ F]FMISO PET imaging. ${ }^{76}$ Therefore it is essential to assess the spatial relationship between hypoxic volumes and the recurrences before adapting radiation treatment plans.

Since tumor hypoxia is a dynamic process involving acute and chronic hypoxia, it is of importance to investigate the effect of hypoxia changes during treatment and its effect on the hypoxia PET based radiation treatment plan. A relative stable spatial distribution of the $\left[{ }^{18} \mathrm{~F}\right]$ FMISO PET uptake was observed during chemoradiotherapy in 16 patients with locally advanced head and neck cancer. ${ }^{112}$ While Lin et al. observed a change in the hypoxic volume in 4 out of 7 patients, resulting in decreased radiation coverage based on the initial hypoxia PET based treatment plan. ${ }^{113}$ Nevertheless, hypoxia dose painting still increased the equivalent uniform dose to the hypoxic volume. This dose could however be further increased by incorporating serial hypoxia PET imaging in the radiotherapy plan. These results were confirmed by a study using $\left[{ }^{18}\right.$ F]FAZA PET imaging, before and during radiation therapy, here also fluctuations of hypoxia were observed during treatment and adaptive radiotherapy was recommended. ${ }^{101}$

Besides the use for radiotherapy, hypoxia PET imaging could provide useful information for the selection of patients for hypoxia-targeted drugs. The use of $\left[{ }^{18} \mathrm{~F}\right] \mathrm{FMISO}$ during hypoxia targeted therapy with tirapazamine in combination with chemoradiation was investigated in 16 patients with head and neck cancer. ${ }^{114}$ All tumor sites showed a reduced uptake after 4 weeks of treatment with chemoradiation and tirapazamine, which was consistent with an effect after hypoxia-targeted therapy. These results were confirmed by a later study which showed that $\left[{ }^{18} \mathrm{~F}\right]$ FMISO PET imaging is associated with a higher risk of locoregional failure in patients treated with (standard) chemo-radiation. In patients with a hypoxic tumor the locoregional control could be improved by additional treatment with tirapazamine, while in patients without tumor hypoxia additional therapy with tirapa- 
zamine was not effective. This study demonstrates that $\left[{ }^{18} \mathrm{~F}\right] \mathrm{FMISO}$ may identify patients who may benefit from hypoxia targeting drugs. ${ }^{82}$

At the moment there is no literature available regarding the use of hypoxia PET imaging with nitroimidazoles for hypoxia targeting in patients with lung cancer. However there is an ongoing trial investigating the effect of nitroglycerin (a nitric oxide donor, known as a vasodilating agent) on $\left[{ }^{18} \mathrm{~F}\right] \mathrm{HX} 4$ hypoxia PET imaging (NCT01210378). This trial is an example of a new interesting approach of 'window-of-opportunity' clinical trials, to gain insights about the antitumor activity of nitroglycerin or for example other hypoxia modifiers in a disease state not disturbed by previous or simultaneous treatments. In addition a terminated trial is reported (NCT00862134), where patients were treated with the hypoxia activated drug PR104, in combination with $\left[{ }^{18} \mathrm{~F}\right] \mathrm{FMISO}$ PET imaging. For patients with renal cell carcinoma, $\left[{ }^{18} \mathrm{~F}\right]$ FMISO PET imaging was used to assess the changes of tumor hypoxia after treatment with sunitinib. In this study patients with baseline tumor hypoxia showed a decreased $\left[{ }^{18} \mathrm{~F}\right]$ FMISO uptake during treatment with sunitinib, while the $\left[{ }^{18} \mathrm{~F}\right] \mathrm{FMISO}$ uptake remained stable in the non-hypoxic lesions. ${ }^{92}$

To our knowledge no other hypoxia-targeted drugs were investigated in combination with hypoxia PET imaging. However, we believe that the addition of hypoxia PET imaging to the currently investigated antihypoxia treatments is essential. In this way patients and acquired results can be stratified based on the presence of tumor hypoxia, creating the evidence for future patient selection.

\section{FUTURE PERSPECTIVES}

Several nitroimidazole-based PET tracers have shown their ability not only to reliably measure tumor hypoxia but also demonstrated the prognostic significance for treatment outcome in several cancers. Preclinical and clinical studies confirm a value of PET in monitoring important changes in tumor hypoxia during treatment and show the feasibility of integrating hypoxia PET imaging for patient's stratification, therapy optimization before initiation of treatment and/or during therapy. Although the results of the clinical studies incorporating hypoxia PET imaging are promising, none of the hypoxia PET tracers have yet been adequately validated to justify its use in routine clinical practice. Prospective randomized clinical trials, integrating hypoxia PET imaging, testing relevant interventions and evaluating dose escalation strategies are therefore necessary.

\section{ACKNOWLEDGEMENTS}

Authors acknowledge financial support from the QulC-ConCePT project, which is partly funded by EFPI A companies and the Innovative Medicine Initiative Joint Undertaking (IMI JU) under Grant Agreement No. 115151. Authors also acknowledge financial support from the National Institute of Health (NIH-USA U01 CA 143062-01, Radiomics of NSCLC), EU 6th 
(Euroxy no: 502932) and 7th framework program (Metoxia no: 222741 and ARTFORCE no: 257144), euroCAT (IVA Interreg - www.eurocat.info), Kankeronderzoekfonds Limburg from the Health Foundation Limburg and the Dutch Cancer Society (KWF UM 2008-4210, KWF UM 2009-4454, KWF UM 2011-5020, KWF MAC 2011-4970, KWF UM 2012-5394, KWF MAC 2013-6425).

\section{REFERENCES}

1. Nordsmark M, Overgaard J A confirmatory prognostic study on oxygenation status and loco-regional control in advanced head and neck squamous cell carcinoma treated by radiation therapy. Radiother Oncol 2000;57:39-43.

2. Rasey JS, Koh WJ, Evans ML, Peterson LM, Lewellen TK, Graham MM et al. Quantifying regional hypoxia in human tumors with positron emission tomography of [18F]fluoromisonidazole: a pretherapy study of 37 patients. Int J Radiat Oncol Biol Phys 1996;36:417-28.

3. Krohn KA, Link JM, Mason RP Molecular imaging of hypoxia. J Nucl Med 2008;49 Suppl 2:129S-48S.

4. Gronroos T, Eskola O, Lehtio K, Minn H, Marjamaki P, Bergman J et al. Pharmacokinetics of [18F]FETNIM: a potential marker for PET. J Nucl Med 2001;42:1397-404.

5. Lord EM, Harwell L, Koch CJ Detection of Hypoxic Cells by Monoclonal-Antibody Recognizing 2-Nitroimidazole Adducts. Cancer Res 1993;53:5721-6.

6. Dolbier WR, Jr., Li AR, Koch CJ, Shiue CY, Kachur AV [18F]-EF5, a marker for PET detection of hypoxia: synthesis of precursor and a new fluorination procedure. Appl Radiat Isot 2001;54:73-80.

7. Kolb HC, Finn MG, Sharpless KB Click Chemistry: Diverse Chemical Function from a Few Good Reactions. Angew Chem Int Ed Engl 2001;40:2004-21.

8. Doss $\mathrm{M}$, Zhang JJ, Belanger MJ, Stubbs JB, Hostetler ED, Alpaugh $\mathrm{K}$ et al. Biodistribution and radiation dosimetry of the hypoxia marker $18 \mathrm{~F}-\mathrm{HX} 4$ in monkeys and humans determined by using whole-body PET/CT. Nucl Med Commun 2010;31:1016-24.

9. Lopci E, Grassi I, Chiti A, Nanni C, Cicoria G, Toschi L et al. PET radiopharmaceuticals for imaging of tumor hypoxia: a review of the evidence. Am J Nucl Med Mol Imaging 2014;4:365-84.

10. Piert $M$, Machulla $H$, Becker $G$, Stahlschmidt A, Patt $M$, Aldinger $P$ et al. Introducing fluorine-18 fluoromisonidazole positron emission tomography for the localisation and quantification of pig liver hypoxia. Eur J Nucl Med 1999;26:95-109.

11. Bentzen L, Keiding S, Horsman MR, Gronroos T, Hansen SB, Overgaard J Assessment of hypoxia in experimental mice tumours by [18F]fluoromisonidazole PET and pO2 electrode measurements. Influence of tumour volume and carbogen breathing. Acta Oncol 2002;41:304-12.

12. Dubois L, Landuyt W, Haustermans K, Dupont P, Bormans G, Vermaelen P et al. Evaluation of hypoxia in an experimental rat tumour model by 
[(18)F]fluoromisonidazole PET and immunohistochemistry. Brit J Cancer 2004;91: 1947-54.

13. Troost EG, Laverman P, Philippens ME, Lok J, van der Kogel AJ, Oyen WJ et al. Correlation of [18F]FMISO autoradiography and pimonidazole [corrected] immunohistochemistry in human head and neck carcinoma xenografts. Eur J Nucl Med Mol Imaging 2008;35:1803-11.

14. Cho H, Ackerstaff E, Carlin S, Lupu ME, Wang Y, Rizwan A et al. Noninvasive multimodality imaging of the tumor microenvironment: registered dynamic magnetic resonance imaging and positron emission tomography studies of a preclinical tumor model of tumor hypoxia. Neoplasia 2009;11:247-59, 2p following 59.

15. Huang T, Civelek AC, Zheng H, Ng CK, Duan X, Li J et al. (18)F-misonidazole PET imaging of hypoxia in micrometastases and macroscopic xenografts of human nonsmall cell lung cancer: a correlation with autoradiography and histological findings. Am J Nucl Med Mol Imaging 2013;3:142-53.

16. Matsumoto K, Szajek L, Krishna MC, Cook JA, Seidel J, Grimes K et al. The influence of tumor oxygenation on hypoxia imaging in murine squamous cell carcinoma using [64Cu]Cu-ATSM or [18F]Fluoromisonidazole positron emission tomography. Int J Oncol 2007;30:873-81.

17. Bruehlmeier M, Kaser-Hotz B, Achermann R, Bley CR, Wergin M, Schubiger PA et al. Measurement of tumor hypoxia in spontaneous canine sarcomas. Vet Radiol Ultrasound 2005;46:348-54.

18. O'Donoghue JA, Zanzonico P, Pugachev A, Wen B, Smith-Jones P, Cai S et al. Assessment of regional tumor hypoxia using $18 \mathrm{~F}$-fluoromisonidazole and $64 \mathrm{Cu}(\mathrm{II})-$ diacetyl-bis(N4-methylthiosemicarbazone) positron emission tomography: Comparative study featuring microPET imaging, Po2 probe measurement, autoradiography, and fluorescent microscopy in the R3327-AT and FaDu rat tumor models. Int J Radiat Oncol Biol Phys 2005;61:1493-502.

19. Chang J, Wen B, Kazanzides P, Zanzonico P, Finn RD, Fichtinger $G$ et al. A robotic system for 18F-FMISO PET-guided intratumoral pO2 measurements. Med Phys 2009;36:5301-9.

20. Monnich D, Troost EG, Kaanders JH, Oyen WJ, Alber M, Zips D et al. Correlation between tumor oxygenation and 18F-fluoromisonidazole PET data simulated based on microvessel images. Acta Oncol 2013;52:1308-13.

21. Mortensen LS, Buus S, Nordsmark M, Bentzen L, Munk OL, Keiding S et al. Identifying hypoxia in human tumors: A correlation study between 18F-FMISO PET and the Eppendorf oxygen-sensitive electrode. Acta Oncol 2010;49:934-40.

22. Gagel B, Piroth M, Pinkawa M, Reinartz P, Zimny M, Kaiser HJ et al. pO polarography, contrast enhanced color duplex sonography (CDS), [18F] fluoromisonidazole and [18F] fluorodeoxyglucose positron emission tomography: validated methods for the evaluation of therapy-relevant tumor oxygenation or only bricks in the puzzle of tumor hypoxia? BMC Cancer 2007;7:113.

23. Zimny M, Gagel B, DiMartino E, Hamacher K, Coenen HH, Westhofen M et al. FDG--a marker of tumour hypoxia? A comparison with [18F]fluoromisonidazole and pO2polarography in metastatic head and neck cancer. Eur J Nucl Med Mol Imaging 2006;33:1426-31. 
24. Bentzen L, Keiding S, Nordsmark M, Falborg L, Hansen SB, Keller J et al. Tumour oxygenation assessed by $18 \mathrm{~F}$-fluoromisonidazole PET and polarographic needle electrodes in human soft tissue tumours. Radiother Oncol 2003;67:339-44.

25. Norikane T, Yamamoto Y, Maeda Y, Kudomi N, Matsunaga T, Haba R et al. Correlation of (18)F-fluoromisonidazole PET findings with HIF-1alpha and p53 expressions in head and neck cancer: comparison with (18)F-FDG PET. Nucl Med Commun 2014;35:30-5.

26. Sato J, Kitagawa Y, Yamazaki Y, Hata H, Asaka T, Miyakoshi M et al. Advantage of FMISO-PET over FDG-PET for predicting histological response to preoperative chemotherapy in patients with oral squamous cell carcinoma. Eur J Nucl Med Mol Imaging 2014.

27. Cherk MH, Foo SS, Poon AM, Knight SR, Murone C, Papenfuss AT et al. Lack of correlation of hypoxic cell fraction and angiogenesis with glucose metabolic rate in non-small cell lung cancer assessed by 18 F-Fluoromisonidazole and 18F-FDG PET. J Nucl Med 2006;47:1921-6.

28. Cher LM, Murone C, Lawrentschuk N, Ramdave S, Papenfuss A, Hannah A et al. Correlation of hypoxic cell fraction and angiogenesis with glucose metabolic rate in gliomas using 18F-fluoromisonidazole, 18F-FDG PET, and immunohistochemical studies. J Nucl Med 2006;47:410-8.

29. Cheng J, Lei L, Xu J, Sun Y, Zhang Y, Wang X et al. 18F-fluoromisonidazole PET/CT: a potential tool for predicting primary endocrine therapy resistance in breast cancer. $J$ Nucl Med 2013;54:333-40.

30. Lawrentschuk N, Poon AM, Foo SS, Putra LG, Murone C, Davis ID et al. Assessing regional hypoxia in human renal tumours using 18F-fluoromisonidazole positron emission tomography. BJU Int 2005;96:540-6.

31. Busk M, Horsman MR, Jakobsen S, Bussink J, van der Kogel A, Overgaard J Cellular uptake of PET tracers of glucose metabolism and hypoxia and their linkage. Eur J Nucl Med Mol Imaging 2008;35:2294-303.

32. Busk M, Horsman MR, Jakobsen S, Keiding S, van der Kogel AJ, Bussink J et al. Imaging hypoxia in xenografted and murine tumors with $18 \mathrm{~F}$-fluoroazomycin arabinoside: a comparative study involving microPET, autoradiography, PO2polarography, and fluorescence microscopy. Int J Radiat Oncol Biol Phys 2008;70:1202-

33. Busk M, Horsman MR, Jakobsen S, Hansen KV, Bussink J, van der Kogel A et al. Can hypoxia-PET map hypoxic cell density heterogeneity accurately in an animal tumor model at a clinically obtainable image contrast? Radiother Oncol 2009;92:429-36.

34. Busk M, Toustrup K, Sorensen BS, Alsner J, Horsman MR, Jakobsen S et al. In vivo identification and specificity assessment of mRNA markers of hypoxia in human and mouse tumors. BMC Cancer 2011;11:63.

35. Carlin S, Zhang H, Reese M, Ramos NN, Chen Q, Ricketts SA A comparison of the imaging characteristics and microregional distribution of 4 hypoxia PET tracers. J Nucl Med 2014;55:515-21.

36. Tran LB, Bol A, Labar D, Jordan B, Magat J, Mignion L et al. Hypoxia imaging with the nitroimidazole 18F-FAZA PET tracer: a comparison with OxyLite, EPR oximetry and 19F-MRI relaxometry. Radiother Oncol 2012;105:29-35. 
37. Lo Dico A, Valtorta S, Martelli C, Belloli S, Gianelli U, Tosi D et al. Validation of an engineered cell model for in vitro and in vivo HIF-1alpha evaluation by different imaging modalities. Mol Imaging Biol 2014;16:210-23.

38. Yang DJ, Wallace S, Cherif A, Li C, Gretzer MB, Kim EE et al. Development of F-18labeled fluoroerythronitroimidazole as a PET agent for imaging tumor hypoxia. Radiology 1995;194:795-800.

39. Hu M, Xing L, Mu D, Yang W, Yang G, Kong L et al. Hypoxia imaging with 18Ffluoroerythronitroimidazole integrated PET/CT and immunohistochemical studies in non-small cell lung cancer. Clin Nucl Med 2013;38:591-6.

40. Chitneni SK, Bida GT, Dewhirst MW, Zalutsky MR A simplified synthesis of the hypoxia imaging agent 2-(2-Nitro-1H-imidazol-1-yl)-N-(2,2,3,3,3-[(18)F]pentafluoropropyl)-acetamide ([18F]EF5). Nucl Med Biol 2012;39:1012-8.

41. Ziemer LS, Evans SM, Kachur AV, Shuman AL, Cardi CA, Jenkins WT et al. Noninvasive imaging of tumor hypoxia in rats using the 2-nitroimidazole 18F-EF5. Eur J Nucl Med Mol Imaging 2003;30:259-66.

42. Chitneni SK, Bida GT, Zalutsky MR, Dewhirst MW Comparison of the Hypoxia PET Tracer 18F-EF5 to Immunohistochemical Marker EF5 in 3 Different Human Tumor Xenograft Models. J Nucl Med 2014;55:1192-7.

43. Silen J, Hogel H, Kivinen K, Silvoniemi A, Forsback S, Loyttyniemi E et al. Uptake of [F]EF5 as a Tracer for Hypoxic and Aggressive Phenotype in Experimental Head and Neck Squamous Cell Carcinoma. Transl Oncol 2014.

44. Mahy P, De Bast M, Leveque PH, Gillart J, Labar D, Marchand J et al. Preclinical validation of the hypoxia tracer 2-(2-nitroimidazol-1-yl)- N-(3,3,3-[(18)F]trifluoropropyl)acetamide, [(18)F]EF3. Eur J Nucl Med Mol Imaging 2004;31:1263-72.

45. Mahy P, De Bast M, Gillart J, Labar D, Gregoire V Detection of tumour hypoxia: comparison between EF5 adducts and [18F]EF3 uptake on an individual mouse tumour basis. Eur J Nucl Med Mol Imaging 2006;33:553-6.

46. Christian N, Bol A, De Bast M, Labar D, Lee J, Mahy P et al. Determination of tumour hypoxia with the PET tracer [18F]EF3: improvement of the tumour-to-background ratio in a mouse tumour model. Eur J Nucl Med Mol Imaging 2007;34:1348-54.

47. Dubois L, Lieuwes NG, Janssen MH, Peeters WJ, Windhorst AD, Walsh JC et al. Preclinical evaluation and validation of [18F]HX4, a promising hypoxia marker for PET imaging. Proc Natl Acad Sci U S A 2011;108:14620-5.

48. Rademakers SE, Lok J, van der Kogel AJ, Bussink J, Kaanders JH Metabolic markers in relation to hypoxia; staining patterns and colocalization of pimonidazole, HIF-1alpha, CAIX, LDH-5, GLUT-1, MCT1 and MCT4. BMC Cancer 2011;11:167.

49. Sorger $D$, Patt $M$, Kumar $P$, Wiebe LI, Barthel $H$, Seese $A$ et al. [18F]Fluoroazomycinarabinofuranoside (18FAZA) and [18F]Fluoromisonidazole (18FMISO): a comparative study of their selective uptake in hypoxic cells and PET imaging in experimental rat tumors. Nucl Med Biol 2003;30:317-26.

50. Piert M, Machulla HJ, Picchio M, Reischl G, Ziegler S, Kumar P et al. Hypoxia-specific tumor imaging with 18F-fluoroazomycin arabinoside. J Nucl Med 2005;46:106-

51. Reischl G, Dorow DS, Cullinane C, Katsifis A, Roselt P, Binns D et al. Imaging of tumor hypoxia with [124I]IAZA in comparison with [18F]FMISO and [18F]FAZA--first small animal PET results. J Pharm Pharm Sci 2007;10:203-11. 
52. Maier FC, Kneilling M, Reischl G, Cay F, Bukala D, Schmid A et al. Significant impact of different oxygen breathing conditions on noninvasive in vivo tumor-hypoxia imaging using [(1)(8)F]-fluoro-azomycinarabino-furanoside ([(1)(8)F]FAZA). Radiat Oncol 2011;6:165.

53. Gronroos T, Bentzen L, Marjamaki P, Murata R, Horsman MR, Keiding S et al. Comparison of the biodistribution of two hypoxia markers [18F]FETNIM and [18F]FMISO in an experimental mammary carcinoma. Eur J Nucl Med Mol Imaging 2004;31:513-20.

54. Dubois L, Landuyt W, Cloetens L, Bol A, Bormans G, Haustermans K et al. [18F]EF3 is not superior to [18F]FMISO for PET-based hypoxia evaluation as measured in a rat rhabdomyosarcoma tumour model. Eur J Nucl Med Mol Imaging 2009;36:209-18.

55. Mahy P, De Bast M, de Groot T, Cheguillaume A, Gillart J, Haustermans K et al. Comparative pharmacokinetics, biodistribution, metabolism and hypoxia-dependent uptake of [18F]-EF3 and [18F]-MISO in rodent tumor models. Radiother Oncol 2008;89:353-60.

56. Peeters SG, Zegers CM, Lieuwes NG, Van Elmpt W, Eriksson J, Van Dongen GA et al. A comparative study of [18F]HX4, [18F]FAZA and [18F]FMISO hypoxia PET tracers in a preclinical tumor model. Int J Radiat Oncol Biol Phys 2014.

57. Chen L, Zhang Z, Kolb HC, Walsh JC, Zhang J, Guan Y (1)(8)F-HX4 hypoxia imaging with PET/CT in head and neck cancer: a comparison with (1)(8)F-FMISO. Nucl Med Commun 2012;33:1096-102.

58. Postema EJ, McEwan AJ, Riauka TA, Kumar P, Richmond DA, Abrams DN et al. Initial results of hypoxia imaging using 1-alpha-D: -(5-deoxy-5-[18F]-fluoroarabinofuranosyl)2-nitroimidazole ( 18F-FAZA). Eur J Nucl Med Mol Imaging 2009;36:1565-73.

59. Trinkaus ME, Blum R, Rischin D, Callahan J, Bressel M, Segard T et al. Imaging of hypoxia with 18F-FAZA PET in patients with locally advanced non-small cell lung cancer treated with definitive chemoradiotherapy. J Med Imaging Radiat Oncol 2013;57:475-

60. Zegers $\mathrm{CM}$, van Elmpt W, Wierts R, Reymen B, Sharifi H, Ollers MC et al. Hypoxia imaging with $[(1)(8) F] H X 4$ PET in NSCLC patients: defining optimal imaging parameters. Radiother Oncol 2013;109:58-64.

61. Szeto MD, Chakraborty G, Hadley J, Rockne R, Muzi M, Alvord EC, Jr. et al. Quantitative metrics of net proliferation and invasion link biological aggressiveness assessed by MRI with hypoxia assessed by FMISO-PET in newly diagnosed glioblastomas. Cancer Res 2009;69:4502-9.

62. Hirata K, Terasaka S, Shiga T, Hattori N, Magota K, Kobayashi H et al. (1)(8)FFluoromisonidazole positron emission tomography may differentiate glioblastoma multiforme from less malignant gliomas. Eur J Nucl Med Mol Imaging 2012;39:76070.

63. Yamamoto $\mathrm{Y}$, Maeda $\mathrm{Y}$, Kawai N, Kudomi N, Aga F, Ono $\mathrm{Y}$ et al. Hypoxia assessed by 18F-fluoromisonidazole positron emission tomography in newly diagnosed gliomas. Nucl Med Commun 2012;33:621-5.

64. Segard T, Robins PD, Yusoff IF, Ee H, Morandeau L, Campbell EM et al. Detection of hypoxia with 18F-fluoromisonidazole (18F-FMISO) PET/CT in suspected or proven pancreatic cancer. Clin Nucl Med 2013;38:1-6. 
65. Loi S, Ngan SY, Hicks RJ, Mukesh B, Mitchell P, Michael M et al. Oxaliplatin combined with infusional 5-fluorouracil and concomitant radiotherapy in inoperable and metastatic rectal cancer: a phase I trial. Brit J Cancer 2005;92:655-61.

66. Havelund BM, Holdgaard PC, Rafaelsen SR, Mortensen LS, Theil J, Bender D et al. Tumour hypoxia imaging with 18F-fluoroazomycinarabinofuranoside PET/CT in patients with locally advanced rectal cancer. Nucl Med Commun 2013;34:155-61.

67. Garcia-Parra R, Wood D, Shah RB, Siddiqui J, Hussain H, Park H et al. Investigation on tumor hypoxia in resectable primary prostate cancer as demonstrated by 18F-FAZA $\mathrm{PET} / \mathrm{CT}$ utilizing multimodality fusion techniques. Eur J Nucl Med Mol Imaging 2011;38:1816-23.

68. Bartlett RM, Beattie BJ, Naryanan M, Georgi JC, Chen Q, Carlin SD et al. Image-guided $\mathrm{PO} 2$ probe measurements correlated with parametric images derived from 18Ffluoromisonidazole small-animal PET data in rats. J Nucl Med 2012;53:1608-15.

69. Busk M, Munk OL, Jakobsen S, Wang T, Skals M, Steiniche T et al. Assessing hypoxia in animal tumor models based on pharmocokinetic analysis of dynamic FAZA PET. Acta Oncol 2010;49:922-33.

70. Wang W, Lee NY, Georgi JC, Narayanan M, Guillem J, Schoder H et al. Pharmacokinetic analysis of hypoxia (18)F-fluoromisonidazole dynamic PET in head and neck cancer. J Nucl Med 2010;51:37-45.

71. Thorwarth D, Eschmann SM, Paulsen F, Alber M A kinetic model for dynamic [18F]Fmiso PET data to analyse tumour hypoxia. Phys Med Biol 2005;50:2209-24.

72. Verwer EE, van Velden FH, Bahce I, Yaqub M, Schuit RC, Windhorst AD et al. Pharmacokinetic analysis of [18F]FAZA in non-small cell lung cancer patients. Eur J Nucl Med Mol Imaging 2013;40:1523-31.

73. Mortensen LS, Busk M, Nordsmark M, Jakobsen S, Theil J, Overgaard J et al. Accessing radiation response using hypoxia PET imaging and oxygen sensitive electrodes: a preclinical study. Radiother Oncol 2011;99:418-23.

74. Beck R, Roper B, Carlsen JM, Huisman MC, Lebschi JA, Andratschke N et al. Pretreatment 18F-FAZA PET predicts success of hypoxia-directed radiochemotherapy using tirapazamine. J Nucl Med 2007;48:973-80.

75. Lehtio K, Eskola O, Viljanen T, Oikonen V, Gronroos T, Sillanmaki L et al. Imaging perfusion and hypoxia with $\mathrm{PET}$ to predict radiotherapy response in head-and-neck cancer. Int J Radiat Oncol Biol Phys 2004;59:971-82.

76. Dirix P, Vandecaveye V, De Keyzer F, Stroobants S, Hermans R, Nuyts S Dose painting in radiotherapy for head and neck squamous cell carcinoma: value of repeated functional imaging with (18)F-FDG PET, (18)F-fluoromisonidazole PET, diffusionweighted MRI, and dynamic contrast-enhanced MRI. J Nucl Med 2009;50:1020-7.

77. Mortensen LS, Johansen J, Kallehauge J, Primdahl H, Busk M, Lassen $P$ et al. FAZA $\mathrm{PET} / \mathrm{CT}$ hypoxia imaging in patients with squamous cell carcinoma of the head and neck treated with radiotherapy: results from the DAHANCA 24 trial. Radiother Oncol 2012;105:14-20.

78. Lee $\mathrm{N}$, Nehmeh S, Schoder H, Fury M, Chan K, Ling CC et al. Prospective trial incorporating pre-/mid-treatment [18F]-misonidazole positron emission tomography for head-and-neck cancer patients undergoing concurrent chemoradiotherapy. Int J Radiat Oncol Biol Phys 2009;75:101-8. 
79. Zips D, Zophel K, Abolmaali N, Perrin R, Abramyuk A, Haase R et al. Exploratory prospective trial of hypoxia-specific PET imaging during radiochemotherapy in patients with locally advanced head-and-neck cancer. Radiother Oncol 2012;105:21-8.

80. Eschmann SM, Paulsen F, Bedeshem C, Machulla HJ, Hehr T, Bamberg M et al. Hypoxia-imaging with (18)F-Misonidazole and PET: changes of kinetics during radiotherapy of head-and-neck cancer. Radiother Oncol 2007;83:406-10.

81. Thorwarth D, Eschmann SM, Scheiderbauer J, Paulsen F, Alber M Kinetic analysis of dynamic 18F-fluoromisonidazole PET correlates with radiation treatment outcome in head-and-neck cancer. BMC Cancer 2005;5:152.

82. Rischin D, Hicks RJ, Fisher R, Binns D, Corry J, Porceddu S et al. Prognostic significance of [18F]-misonidazole positron emission tomography-detected tumor hypoxia in patients with advanced head and neck cancer randomly assigned to chemoradiation with or without tirapazamine: a substudy of Trans-Tasman Radiation Oncology Group Study 98.02. J Clin Oncol 2006;24:2098-104.

83. Rajendran JG, Schwartz DL, O'Sullivan J, Peterson LM, Ng P, Scharnhorst J et al. Tumor hypoxia imaging with [F-18] fluoromisonidazole positron emission tomography in head and neck cancer. Clin Cancer Res 2006;12:5435-41.

84. Komar G, Lehtio K, Seppanen M, Eskola O, Levola H, Lindholm P et al. Prognostic value of tumour blood flow, [(18)F]EF5 and [ (18)F]FDG PET/CT imaging in patients with head and neck cancer treated with radiochemotherapy. Eur J Nucl Med Mol Imaging 2014;41:2042-50.

85. Thorwarth D, Eschmann SM, Holzner F, Paulsen F, Alber M Combined uptake of [18F]FDG and [18F]FMISO correlates with radiation therapy outcome in head-andneck cancer patients. Radiother Oncol 2006;80:151-6.

86. Li L, Hu M, Zhu H, Zhao W, Yang G, Yu J Comparison of 18F-Fluoroerythronitroimidazole and $18 \mathrm{~F}$-fluorodeoxyglucose positron emission tomography and prognostic value in locally advanced non-small-cell lung cancer. Clin Lung Cancer 2010;11:335-40.

87. Eschmann SM, Paulsen F, Reimold M, Dittmann H, Welz S, Reischl G et al. Prognostic impact of hypoxia imaging with 18F-misonidazole PET in non-small cell lung cancer and head and neck cancer before radiotherapy. J Nucl Med 2005;46:253-60.

88. Kawai N, Lin W, Cao WD, Ogawa D, Miyake K, Haba R et al. Correlation between (18)F-fluoromisonidazole PET and expression of HIF-1alpha and VEGF in newly diagnosed and recurrent malignant gliomas. Eur J Nucl Med Mol Imaging 2014;41:1870-8.

89. Swanson KR, Chakraborty G, Wang CH, Rockne R, Harpold HL, Muzi M et al. Complementary but distinct roles for MRI and 18F-fluoromisonidazole PET in the assessment of human glioblastomas. J Nucl Med 2009;50:36-44.

90. Vercellino L, Groheux D, Thoury A, Delord M, Schlageter MH, Delpech Y et al. Hypoxia imaging of uterine cervix carcinoma with (18)F-FETNIM PET/CT. Clin Nucl Med 2012;37:1065-8.

91. Yue J, Yang $Y$, Cabrera AR, Sun X, Zhao S, Xie P et al. Measuring tumor hypoxia with (1)(8)F-FETNIM PET in esophageal squamous cell carcinoma: a pilot clinical study. Dis Esophagus 2012;25:54-61.

92. Hugonnet F, Fournier L, Medioni J, Smadja C, Hindie E, Huchet V et al. Metastatic renal cell carcinoma: relationship between initial metastasis hypoxia, change after 1 
month's sunitinib, and therapeutic response: an 18F-fluoromisonidazole PET/CT study. J Nucl Med 2011;52:1048-55.

93. Oehler C, O'Donoghue JA, Russell J, Zanzonico P, Lorenzen S, Ling CC et al. 18Ffluromisonidazole PET imaging as a biomarker for the response to 5,6dimethylxanthenone-4-acetic acid in colorectal xenograft tumors. J Nucl Med 2011;52:437-44.

94. Valable S, Petit E, Roussel S, Marteau L, Toutain J, Divoux D et al. Complementary information from magnetic resonance imaging and (18)F-fluoromisonidazole positron emission tomography in the assessment of the response to an antiangiogenic treatment in a rat brain tumor model. Nucl Med Biol 2011;38:781-93.

95. Murakami M, Zhao S, Zhao Y, Chowdhury NF, Yu W, Nishijima K et al. Evaluation of changes in the tumor microenvironment after sorafenib therapy by sequential histology and 18F-fluoromisonidazole hypoxia imaging in renal cell carcinoma. Int J Oncol 2012;41:1593-600.

96. Busk M, Mortensen LS, Nordsmark M, Overgaard J, Jakobsen S, Hansen KV et al. PET hypoxia imaging with FAZA: reproducibility at baseline and during fractionated radiotherapy in tumour-bearing mice. Eur J Nucl Med Mol Imaging 2013;40:186-97.

97. Dorow DS, Cullinane C, Conus N, Roselt P, Binns D, McCarthy TJ et al. Multi-tracer small animal PET imaging of the tumour response to the novel pan-Erb-B inhibitor $\mathrm{Cl}$ 1033. Eur J Nucl Med Mol Imaging 2006;33:441-52.

98. Solomon B, Binns D, Roselt P, Weibe LI, McArthur GA, Cullinane C et al. Modulation of intratumoral hypoxia by the epidermal growth factor receptor inhibitor gefitinib detected using small animal PET imaging. Mol Cancer Ther 2005;4:1417-22.

99. Chitneni SK, Bida GT, Yuan H, Palmer GM, Hay MP, Melcher T et al. 18F-EF5 PET imaging as an early response biomarker for the hypoxia-activated prodrug SN30000 combined with radiation treatment in a non-small cell lung cancer xenograft model. J Nucl Med 2013;54:1339-46.

100. Yamane T, Kikuchi M, Shinohara S, Senda M Reduction of [(18)F]fluoromisonidazole uptake after neoadjuvant chemotherapy for head and neck squamous cell carcinoma. Mol Imaging Biol 2011;13:227-31.

101. Servagi-Vernat S, Differding S, Hanin FX, Labar D, Bol A, Lee JA et al. A prospective clinical study of (1)(8)F-FAZA PET-CT hypoxia imaging in head and neck squamous cell carcinoma before and during radiation therapy. Eur J Nucl Med Mol Imaging 2014;41:1544-52.

102. Koh WJ, Bergman KS, Rasey JS, Peterson LM, Evans ML, Graham MM et al. Evaluation of oxygenation status during fractionated radiotherapy in human nonsmall cell lung cancers using [F-18]fluoromisonidazole positron emission tomography. Int J Radiat Oncol Biol Phys 1995;33:391-8.

103. Vera P, Bohn P, Edet-Sanson A, Salles A, Hapdey S, Gardin I et al. Simultaneous positron emission tomography (PET) assessment of metabolism with (1)(8)F-fluoro-2deoxy-d-glucose (FDG), proliferation with (1)(8)F-fluoro-thymidine (FLT), and hypoxia with (1)(8)fluoro-misonidazole (F-miso) before and during radiotherapy in patients with non-small-cell lung cancer (NSCLC): a pilot study. Radiother Oncol 2011;98:109-16.

104. Schuetz M, Schmid MP, Potter R, Kommata S, Georg D, Lukic D et al. Evaluating repetitive 18F-fluoroazomycin-arabinoside (18FAZA) PET in the setting of MRI guided adaptive radiotherapy in cervical cancer. Acta Oncol 2010;49:941-7. 
105. Eary JF, Link JM, Muzi M, Conrad EU, Mankoff DA, White JK et al. Multiagent PET for risk characterization in sarcoma. J Nucl Med 2011;52:541-6.

106. Rajendran JG, Mankoff DA, O'Sullivan F, Peterson LM, Schwartz DL, Conrad EU et al. Hypoxia and glucose metabolism in malignant tumors: evaluation by [18F]fluoromisonidazole and [18F]fluorodeoxyglucose positron emission tomography imaging. Clin Cancer Res 2004;10:2245-52.

107. Overgaard J, Horsman MR Modification of Hypoxia-Induced Radioresistance in Tumors by the Use of Oxygen and Sensitizers. Semin Radiat Oncol 1996;6:10-21.

108. Choi W, Lee SW, Park SH, Ryu JS, Oh SJ, Im KC et al. Planning study for available dose of hypoxic tumor volume using fluorine-18-labeled fluoromisonidazole positron emission tomography for treatment of the head and neck cancer. Radiother Oncol 2010;97:176-82.

109. Hendrickson K, Phillips M, Smith W, Peterson L, Krohn K, Rajendran J Hypoxia imaging with [F-18] FMISO-PET in head and neck cancer: potential for guiding intensity modulated radiation therapy in overcoming hypoxia-induced treatment resistance. Radiother Oncol 2011;101:369-75.

110. Toma-Dasu I, Uhrdin J, Antonovic L, Dasu A, Nuyts S, Dirix P et al. Dose prescription and treatment planning based on FMISO-PET hypoxia. Acta Oncol 2012;51:222-30.

111. Chang JH, Wada M, Anderson NJ, Lim Joon D, Lee ST, Gong SJ et al. Hypoxia-targeted radiotherapy dose painting for head and neck cancer using (18)F-FMISO PET: a biological modeling study. Acta Oncol 2013;52:1723-9.

112. Bittner MI, Wiedenmann N, Bucher S, Hentschel M, Mix M, Weber WA et al. Exploratory geographical analysis of hypoxic subvolumes using 18F-MISO-PET imaging in patients with head and neck cancer in the course of primary chemoradiotherapy. Radiother Oncol 2013;108:511-6.

113. Lin Z, Mechalakos J, Nehmeh S, Schoder H, Lee N, Humm J et al. The influence of changes in tumor hypoxia on dose-painting treatment plans based on 18F-FMISO positron emission tomography. Int J Radiat Oncol Biol Phys 2008;70:1219-28.

114. Hicks RJ, Rischin D, Fisher R, Binns D, Scott AM, Peters LJ Utility of FMISO PET in advanced head and neck cancer treated with chemoradiation incorporating a hypoxiatargeting chemotherapy agent. Eur J Nucl Med Mol Imaging 2005;32:1384-91. 

IMAGING OF TUMOR HYPOXIA 



\section{CHAPTER}

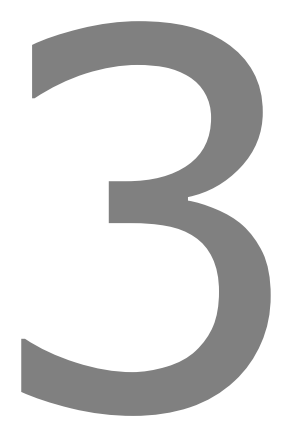

A comparative study of the hypoxia PET tracers $\left[{ }^{18} \mathrm{~F}\right] \mathrm{HX} 4,\left[{ }^{18} \mathrm{~F}\right] \mathrm{FAZA}$ and $\left[{ }^{18} \mathrm{~F}\right] \mathrm{FMISO}$ in a preclinical tumor model

Sarah G.J.A. Peeters, Catharina M.L. Zegers, Natasja G. Lieuwes, Wouter van Elmpt, Jonas Eriksson, Guus A.M.S. van Dongen, Ludwig Dubois*, Philippe Lambin*

Published in: Int J Radiat Oncol Biol Phys. 2015; 91(2):351-9 
56 |Chapter 3

\section{ABSTRACT}

Purpose: Several individual clinical and preclinical studies have shown the possibility to evaluate tumor hypoxia by using noninvasive positron emission tomography (PET). The current study compared 3 hypoxia PET tracers frequently used in the clinic, $\left[{ }^{18} \mathrm{~F}\right] \mathrm{FMISO}$, $\left[{ }^{18} \mathrm{~F}\right] \mathrm{FAZA}$ and $\left[{ }^{18} \mathrm{~F}\right] \mathrm{HX} 4$ in a preclinical tumor model. Tracer uptake was evaluated for the optimal time point for imaging, tumor-to-blood ratios (TBR), spatial reproducibility and sensitivity towards oxygen modifications.

Methods and Materials: PET/computed tomotgraphy (CT) images of rhadomyosarcoma R1 bearing WAG/Rij rats were acquired at multiple time points post injection (p.i.) with one of the hypoxia tracers. TBR values were calculated and reproducibility was investigated by voxel-to-voxel analysis, represented as correlation coefficients (R) or DICE similarity coefficient of the high-uptake volumes. Tumor oxygen modifications were induced by exposure to either carbogen/nicotinamide treatment or $7 \%$ oxygen breathing.

Results: TBR was stabilized and maximal at 2 hours p.i. for $\left[{ }^{18} \mathrm{~F}\right] \mathrm{FAZA}(4.0 \pm 0.5)$ and at 3 hours p.i. for $\left[{ }^{18} \mathrm{~F}\right] \mathrm{HX} 4(7.2 \pm 0.7)$ whereas $\left[{ }^{18} \mathrm{~F}\right] \mathrm{FMISO}$ showed a constant increasing TBR (9.0 \pm 0.8 at 6 hours p.i.). High spatial reproducibility was observed by voxel-to-voxel comparisons and DICE similarity coefficient calculations on the $30 \%$ highest uptake volume for both $\left[{ }^{18} \mathrm{~F}\right] \mathrm{FMISO}(\mathrm{R}=0.86$, DICE coefficient $=0.76)$ and $\left[{ }^{18} \mathrm{~F}\right] \mathrm{HX} 4(\mathrm{R}=0.76$, DICE coefficient $=0.70$ ) whereas $\left[{ }^{18} \mathrm{~F}\right]$ FAZA was less reproducible ( $R=0.52$ DICE coefficient $=0.49$ ). Modifying the hypoxic fraction resulted in enhanced mean standardized uptake values for both $\left[{ }^{18} \mathrm{~F}\right] \mathrm{HX} 4$ and $\left[{ }^{18} \mathrm{~F}\right] \mathrm{FAZA}$ upon $7 \%$ oxygen breathing. Only $\left[{ }^{18} \mathrm{~F}\right] \mathrm{FMISO}$ uptake was found to be reversible upon exposure to nicotinamide and carbogen.

Conclusions: This study indicates that each tracer has its own strengths and, depending on the question to be answered, a different tracer can be put forward. 


\section{INTRODUCTION}

Tumor hypoxia is an important factor in worsening cancer patients' treatment outcome. Regions of low oxygen concentration are a well-known characteristic of solid tumors and can be caused by impaired blood vessel development, temporal occlusions of blood vessels or excessive tumor growth $(1,2)$. Knowledge about the extension and location of hypoxia would provide additional information that could be integrated into strategies of conventional treatments, potentially leading to improved therapeutic outcome (3). Positron Emission Tomography (PET) has been shown to be a suitable, non-invasive, 3dimensional imaging technique for the detection of hypoxic tumor regions. PET tracers containing the oxygen-sensitive nitroimidazole group are specifically designed to detect hypoxic regions, and the feasibility of these tracers has been studied extensively in several independent clinical and preclinical studies (1).

${ }^{18} \mathrm{~F}$ - labeled fluoromisonidazole ( $\left.\left[{ }^{18} \mathrm{~F}\right] \mathrm{FMISO}\right)$ was the first specific hypoxia PET tracer and, although it may be the most frequently used tracer for this purpose, its suitability is limited because of slow tumor-specific accumulation and nonspecific washout (4). Second generation 2-nitroimidazole tracers with different clearance and hydrophilicity characteristics have been developed in an attempt to overcome these disadvantages:, $\left[{ }^{18} \mathrm{~F}\right]$ fluoroazomycin arabinoside (FAZA), $\left[{ }^{18} \mathrm{~F}\right]$ fluoroerythronitroimidazole (FETNIM), and $\left[{ }^{18} \mathrm{~F}\right]$ tri-fluoroetanidazole (EF3). In preclinical settings, these tracers have been investigated separately or solely in comparison to hypoxia immunohistochemical staining or $\left[{ }^{18}\right.$ F]FMISO PET imaging, using different experimental set-ups, tumor models and acquisition protocols (5-8). Next, the third-generation hypoxia tracer $\left[{ }^{18} \mathrm{~F}\right]$ flortanidazole $(\mathrm{HX} 4)$ was developed and evaluated in a preclinical rhabdomyosarcoma tumor model, where it was found to be dependent on tumoral oxygenation status (9). Only recently, a comparative study in preclinical animal models reported a clear relationship among the uptake of $\left[{ }^{18} \mathrm{~F}\right] \mathrm{FMISO},\left[{ }^{18} \mathrm{~F}\right] \mathrm{FAZA}$ and $\left[{ }^{18} \mathrm{~F}\right] \mathrm{HX} 4$ and with immunohistochemical stainings for perfusion, hypoxia and carbonic anhydrase IX (10). With respect to patients usage of PET tumor hypoxia for patient imaging, clinical studies have indicated that both $\left[{ }^{18} \mathrm{~F}\right] \mathrm{FAZA}$ and $\left[{ }^{18} \mathrm{~F}\right] \mathrm{FMISO}$ have prognostic potential $(11,12)$ and a phase I clinical study demonstrated that imaging using $\left[{ }^{18} \mathrm{~F}\right] \mathrm{HX} 4$ was feasible and non-toxic (13).

In this study, we compare the 3 most frequently used and clinically available hypoxia tracers, $\left[{ }^{18} \mathrm{~F}\right] \mathrm{FMISO},\left[{ }^{18} \mathrm{~F}\right] \mathrm{FAZA}$ and $\left[{ }^{18} \mathrm{~F}\right] \mathrm{HX} 4$, along with the metabolic tracer $\left[{ }^{18} \mathrm{~F}\right]$ fluorodeoxyglucose (FDG) in a preclinical tumor model. We investigated the uptake of each tracer over time and determined the tumor-to-background ratios. A second PET scan was performed on the same animal after 48 hours to assess spatial reproducibility. Furthermore, tracer uptake was challenged by exposing the animals to modified oxygen concentrations. The focus of this study was to investigate the performance and characteristics of the different hypoxia PET tracer, using the same tumor model. 


\section{MATERIALS AND METHODS}

\section{Tracer synthesis, tumor model, experimental design}

Tracer synthesis of $\left[{ }^{18} \mathrm{~F}\right] \mathrm{FMISO},\left[{ }^{18} \mathrm{~F}\right] \mathrm{FAZA}$ and $\left[{ }^{18} \mathrm{~F}\right] \mathrm{HX} 4$ (Figure $3.1 \mathrm{~A}$ ) was performed as described previously (14-17). All animal experimental procedures were approved by the Animal Ethical Committee of Maastricht University and were in accordance with the Helsinki Declaration of 1975 , as revised in 2000. Adult WAG/Rij rats received subcutaneous implants of the syngeneic rhabdomyosarcoma R1 tumors $\left(1 \mathrm{~mm}^{3}\right)$ in the lateral flank. Experiments were started when tumors reached a minimal volume of $3 \mathrm{~cm}^{3}$ to meet the resolution of the PET scanner and to have a stable hypoxic (18) and necrotic (5) area. Average tumor volume for $\left[{ }^{18} \mathrm{~F}\right] \mathrm{FDG}=21 \pm 12 \mathrm{~cm}^{3}, 16 \pm 6 \mathrm{~cm}^{3}$ for $\left[{ }^{18} \mathrm{~F}\right] \mathrm{FAZA}, 13 \pm 6 \mathrm{~cm}^{3}$ for $\left[{ }^{18} \mathrm{~F}\right] \mathrm{FMISO}$ and $11 \pm 5 \mathrm{~cm}^{3}$ for $\left[{ }^{18} \mathrm{~F}\right] \mathrm{HX} 4$. During the experimental procedures, rats were anesthetized with intraperitoneal injections of sodium pentobarbital $(60 \mathrm{mg} / \mathrm{kg})$. Animals were immobilized on a board and placed outside the scanner between scans to maintain and monitor anesthesia. Radioactive tracers (radiochemical purity was maintained at $>95 \%$ and synthesis yield at $5.2 \pm 2.5 \mathrm{GBq}$ ) were injected into the lateral tail vein by using an intravenous line ( $0.4 \mathrm{~mm} \mathrm{G27}$ Venoflux needle; Vygon Vet, Ecouen, France) flushed with $10 \%$ heparin solution $\left(21 \pm 2 \mathrm{MBq}\right.$ for $\left[{ }^{18} \mathrm{~F}\right] \mathrm{FDG}, 17 \pm 5 \mathrm{MBq}$ for $\left[{ }^{18} \mathrm{~F}\right] \mathrm{FAZA}, 21 \pm 2 \mathrm{MBq}$ for $\left[{ }^{18} \mathrm{~F}\right] \mathrm{FMISO}$, and $21 \pm 2 \mathrm{MBq}$ for $\left.\left[{ }^{18} \mathrm{~F}\right] \mathrm{HX} 4\right)$.

\section{Image acquisition and analysis}

Images were acquired and analyzed using a clinical PET/CT scanner (Siemens Biograph 40, Siemens Healthcare) and dedicated software (TrueD VC60; Siemens) as described in more detail in the supplement section and previously (9). Tumor to blood ratios (TBR) and tumor to muscle ratios (TMR) were determined using heart and muscle of the hind leg as background tissue, respectively. Spatial reproducibility scans were performed in the same animal within short time frames, using rigid registration voxel to voxel analysis (from 2 to 6 hours post injection [p.i].) or 48 hours apart using nonrigid registration (details in supplement section) of the tumor for long-term comparison to overcome the $24 \%$ tumor growth (which were $31 \pm 2 \%$ for $\left[{ }^{18} \mathrm{~F}\right] \mathrm{FDG}, 26 \pm 2 \%$ for $\left[{ }^{18} \mathrm{~F}\right] \mathrm{FAZA}, 23 \pm 1 \%$ for $\left[{ }^{18} \mathrm{~F}\right] \mathrm{FMISO}$, and $22 \pm 3 \%$ for $\left.\left[{ }^{18} \mathrm{~F}\right] \mathrm{HX} 4\right)$. Furthermore, a voxel-wise comparison of the 2 scans was performed for which a correlation coefficient was calculated. Imaging schedules for oxygen modification using either nicotinamide $\left(500 \mathrm{mg} / \mathrm{kg}\right.$, intraperitoneal) and carbogen $\left(95 \% \mathrm{O}_{2}, 5 \% \mathrm{CO}_{2}\right.$, flow $5 \mathrm{l} / \mathrm{min}$ ) or $7 \%$ oxygen (residual $\mathrm{N}_{2}$, flow $2,5 \mathrm{l} / \mathrm{min}$ ) breathing are shown in Figure 3.1B. In short, after injection of the tracer, a first basal scan was performed at 2 hours p.i., followed by oxygen modification treatment and a second scan at 5 hours p.i.

\section{Statistics}

Prism version 5.01 software (GraphPad) for Windows (Microsoft) was used to perform statistical analyses. To determine the statistical significance of differences between 2 independent groups of variables, we used the nonparametric Mann-Whitney- $U$ test for small groups. Spatial reproducibility was analyzed using either a DICE similarity coefficient 
for the calculation of the overlap fractions or Pearson correlation for the voxel wise comparison. P-values of $<0.05$ were assumed to be significant.

A<smiles>O=[N+]([O-])c1nccn1CC(O)CF</smiles><smiles>O=[N+]([O-])c1nccn1C1OC(CF)C(O)C1O</smiles>

$\left[{ }^{18 \mathrm{~F}}\right] \mathrm{HX} 4$

[ ${ }^{18 \mathrm{~F}] F D G}$<smiles>O=[N+]([O-])c1nccn1Cc1cnnn1C(CO)CF</smiles><smiles>OCC1OC(O)C(F)C(O)C1O</smiles>

Clearance

hepatobiliary

renal

renal

Hydrophilicity

$-0.40$

0.04

$-0.69$

$(\log P)$

B

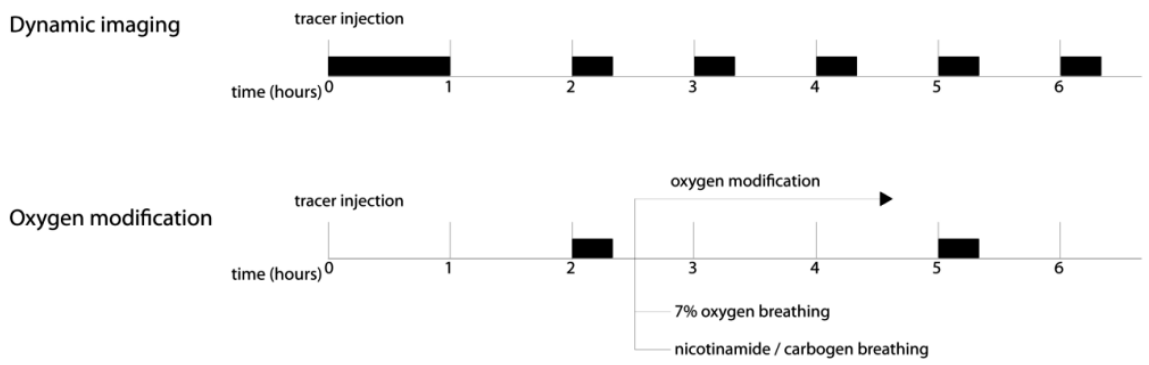

Figure 3.1: Tracer characteristics and imaging protocols. A) Structure formula, main clearance system and logP value for hydrophilicity of the 3 hypoxia tracers $\left.\left[{ }^{18} \mathrm{~F}\right] \mathrm{FMISO},{ }^{18} \mathrm{~F}\right] \mathrm{FAZA}$ and $\left[{ }^{18} \mathrm{~F}\right] \mathrm{HX} 4$ and the metabolic tracer $\left[{ }^{18} \mathrm{~F}\right] F D G(9)$. B) Schematic representation of the different imaging protocols for dynamic imaging and for oxygen modification, using either nicotinamide combined with carbogen breathing or $7 \%$ oxygen breathing. Imaging acquisition is indicated by black boxes. 
A

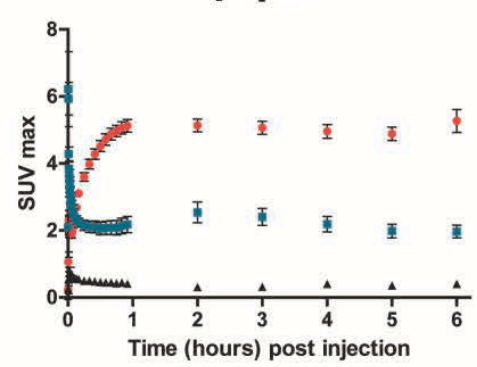

$\left[{ }^{18}\right.$ F]FMISO

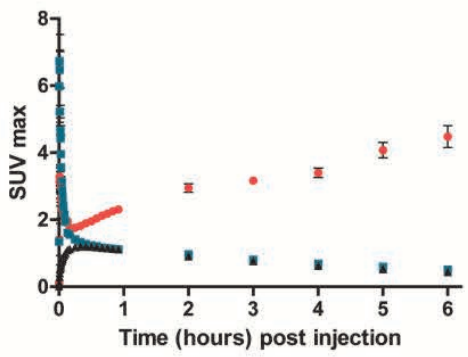

B

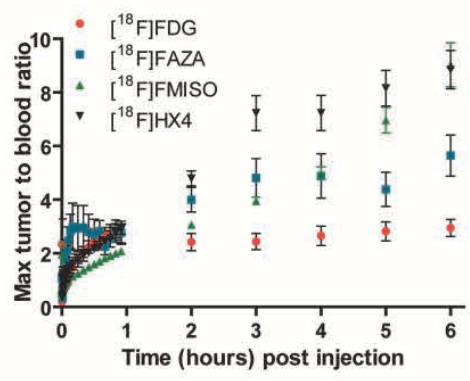

C

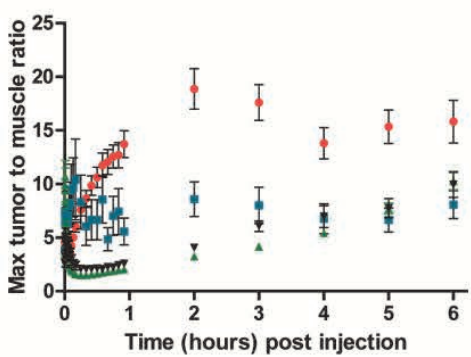

$\left[{ }^{18}\right.$ F]FAZA

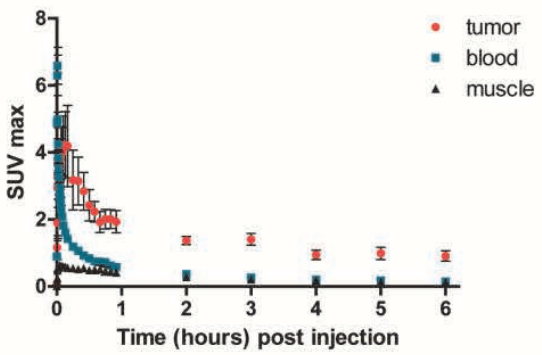

$\left[{ }^{18} \mathrm{~F}\right] \mathrm{HX} 4$
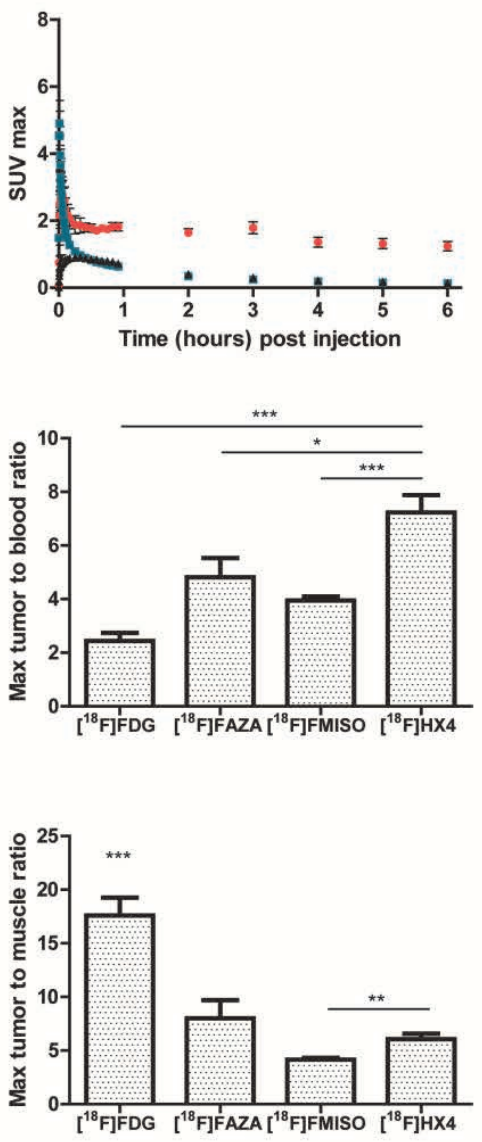

Figure 3.2: SUV $V_{\max }$ and TBR. A) SUV $V_{\max }$ of the tumor (red), blood (blue) and muscle (black) over time. B) Left panel: Maximum TBR over time for $\left[{ }^{18} \mathrm{~F}\right] F D G\left(\right.$ red), $\left[{ }^{18} \mathrm{~F}\right] F A Z A$ (blue), $\left[{ }^{18} \mathrm{~F}\right] F M I S O$ (green) and $\left[{ }^{18} \mathrm{~F}\right] \mathrm{HX} 4$ (black). Right panel; $T B R$ tracer comparison at 3 hours post-injection. ${ }^{*} P<0.05,{ }^{* * *} P<0.001$. C) Left panel: Maximum TMR over time. Right panel: Maximum TMR comparison for the 4 tracers at 3 hours p.i. Number of animals for all experiments: $\left[^{18}\right.$ FJFDG $\left.n=12,{ }^{18} \mathrm{~F}\right] F A Z A n=13,\left[{ }^{18}\right.$ FJFMISO $n=16$ and $\left[^{18} \mathrm{~F}\right] H X 4 n=18$ except for Figure $C\left[{ }^{18} \mathrm{~F}\right] F A Z A n=12$. Data are means $\pm S E M$. SUV $V_{\text {max }}=$ maximum standard uptake value; TBR=tumor-to-blood ratio; TMR=tumor-to-muscle ratio. 


\section{RESULTS}

$\mathrm{PET} / \mathrm{CT}$ imaging was performed to assess tracer accumulation over time for the 4 different tracers, using a dynamic imaging schedule (Figure 3.1A, B). Each tracer had a different

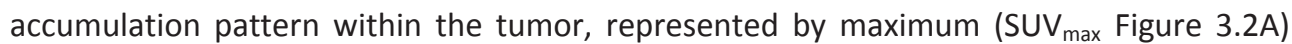
and mean ( $\mathrm{SUV}_{\text {mean }}$ Figure S3.1A) standardized uptake values, whereas blood and muscle tracer uptake exhibited a similar pattern. Due to clearance of the nonspecific binding and specific trapping in hypoxic tumor regions, all TBRs were greater than 1 (Figure 3.2B and Figure S3.1B). Furthermore, clearance rates for heart and muscle were comparable, giving a muscle to blood ratio around unity (Figure S3.1D). For the metabolic tracer $\left[{ }^{18} \mathrm{~F}\right] \mathrm{FDG}$, the maximal TBR of $2.4 \pm 0.3$ was reached at 2 hours p.i. Of all the hypoxia tracers, $\left[{ }^{18} \mathrm{~F}\right] \mathrm{FAZA}$ was the first to reach a plateau phase for TBR at 2 hours p.i. $(4.0 \pm 0.5)$, followed by $\left[{ }^{18} \mathrm{~F}\right] \mathrm{HX} 4$ (TBR 7.2 \pm 0.7 ) at 3 hours p.i. (Figure 3.2B). TBR for $\left[{ }^{18} \mathrm{~F}\right] \mathrm{FMISO}$ kept increasing; TBR of $\left[{ }^{18} \mathrm{~F}\right] \mathrm{FMISO}$ at 6 hours p.i. was comparable to that of $\left[{ }^{18} \mathrm{~F}\right] \mathrm{HX} 4$ at 3 hours p.i. At the first stable time point for $\left[{ }^{18} \mathrm{~F}\right] \mathrm{HX} 4$ (3 hours p.i.), this tracer had a significantly higher TBR than either $\left[{ }^{18} \mathrm{~F}\right]$ FAZA $(\mathrm{P}=0.0154)$ or $\left[{ }^{18} \mathrm{~F}\right] \mathrm{FMISO}(\mathrm{P}<0.0001)$ (Figure $3.2 \mathrm{~B}$ right panel) even at 2 hours p.i. $\left[{ }^{18} \mathrm{~F}\right] \mathrm{HX} 4$ had already reached a TBR that was equal to or higher than that of $\left[{ }^{18} \mathrm{~F}\right]$ FMISO or $\left[{ }^{18} \mathrm{~F}\right]$ FAZA. When muscle tissue was used as tissue reference, trends were shown for the hypoxia tracers that were similar to maximal TBR (Figure 3.2C and Figure S3.1C).

Uptake images from 2, 3, 4 and 5 hours p.i. were compared to the 6 hours p.i. scan to perform a voxel-to-voxel comparison of the absolute tumor uptake. A correlation coefficient was calculated from the 2 scans (see Figure 3.3A). Averaged correlation coefficients demonstrated a stable uptake pattern in the tumor for all investigated tracers over short time periods (up to 6 hours) (Figure 3.3B). Reproducibility was studied by comparing 2 PET scans acquired within a 48 hour time interval using voxel-to-voxel analyses. Calculated correlation coefficients were high for $\left[{ }^{18} \mathrm{~F}\right] \mathrm{FDG}(0.87),\left[{ }^{18} \mathrm{~F}\right] \mathrm{FMISO}(0.86)$ and $\left[{ }^{18} \mathrm{~F}\right] \mathrm{HX} 4$ (0.76); whereas $\left[{ }^{18} \mathrm{~F}\right] \mathrm{FAZA}$ had a significantly $(\mathrm{P}<0.05)$ lower correlation coefficient $(0.52)$ (Figure 3.3C). To further investigate spatial reproducibility, we calculated an overall DICE similarity coefficient in which the high uptake region as a percentage of the total tumor volume from the first scan was compared to the same percentage of total volume area from a second scan (Figure 3.3D). This analysis showed a high reproducibility for $\left[{ }^{18} \mathrm{~F}\right] \mathrm{FDG}$ (0.83), $\left[{ }^{18} \mathrm{~F}\right] \mathrm{FMISO}(0.85),\left[{ }^{18} \mathrm{~F}\right] \mathrm{HX} 4(0.79)$ and $\left[{ }^{18} \mathrm{~F}\right] \mathrm{FAZA}(0.71)$ in a comparison of the $50 \%$ tumor volume with the highest tracer uptake. However, when only the $10 \%$ highest uptake of the tumor volume was selected, $\left[{ }^{18} \mathrm{~F}\right] \mathrm{FDG},\left[{ }^{18} \mathrm{~F}\right] \mathrm{FMISO}$ and $\left[{ }^{18} \mathrm{~F}\right] \mathrm{HX} 4$ showed high spatial reproducibility $\left(0.65,0.59\right.$ and 0.49 , respectively), whereas $\left[{ }^{18} \mathrm{~F}\right] \mathrm{FAZA}$ showed a significant lower $(p<0.05)$ spatial reproducibility of 0.14 (Figure $3.3 \mathrm{E})$. 
62 |Chapter 3

A

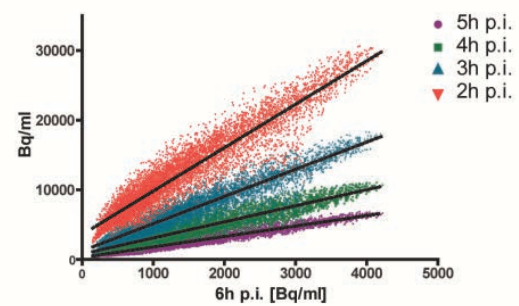

C

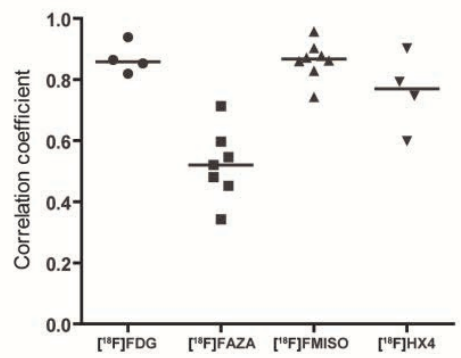

$\mathrm{E}$

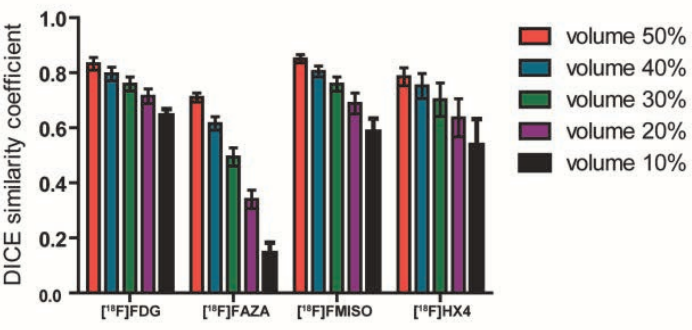

B

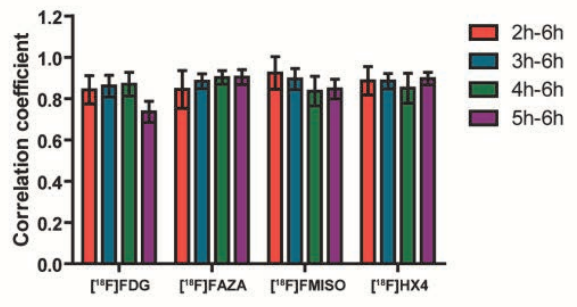

D

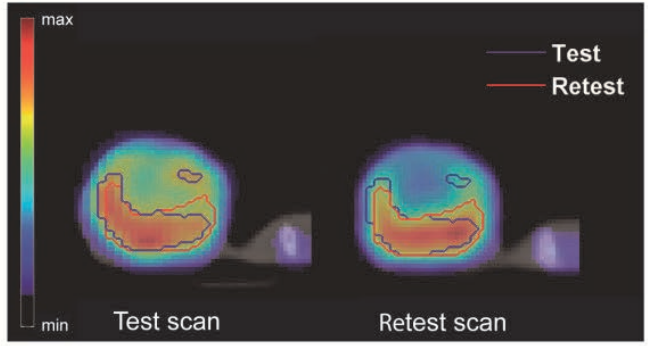

Figure 3.3: Voxel-to-voxel analysis and spatial reproducibility. A) Dynamic scans were used for voxel-to-voxel comparison between scans obtained at 2, 3, 4 or 5 hours p.i. and the 6 hour scan, shown in the representative scatter plot. B) Correlation coefficients of voxel-to-voxel analyses over a short-term time frame. For all tracers $n=4$ C) Correlation coefficients of voxel-to-voxel analyses over a 48 -hour time frame shows [ $\left.{ }^{18} \mathrm{~F}\right] \mathrm{FAZA}$ that is significantly lower: $P_{F D G}$ vs. FAZA $=0.0061, P_{F A Z A}$ vs. FMISO $=0.0003, P_{F A Z A}$ vs HXA $=0.0121$. Each dot represents 1 animal, and the mean is indicated. D) Representative $\left.{ }^{18}{ }^{18} \mathrm{~F}\right] \mathrm{HX} 4$ PET/CT image of a tumor cross-section visualized over a 48-hour time interval. Delineation on the test and retest scan shows the $30 \%$ of the total tumor volume with the highest SUV. Overlap fractions of these regions were calculated and represent the DICE similarity coefficient. E) Spatial reproducibility over a 48-hour time frame is presented per tracer as a percentage of total tumor volume. $\left[^{18} \mathrm{~F}\right] F D G$ $n=4,\left[{ }^{18} \mathrm{~F}\right] F A Z A \quad n=7,\left[{ }^{18} \mathrm{~F}\right] \mathrm{FMISO} n=8$ and $\left[{ }^{18} \mathrm{~F}\right] H X 4 n=4$. Data are the means \pm SEM. 
A
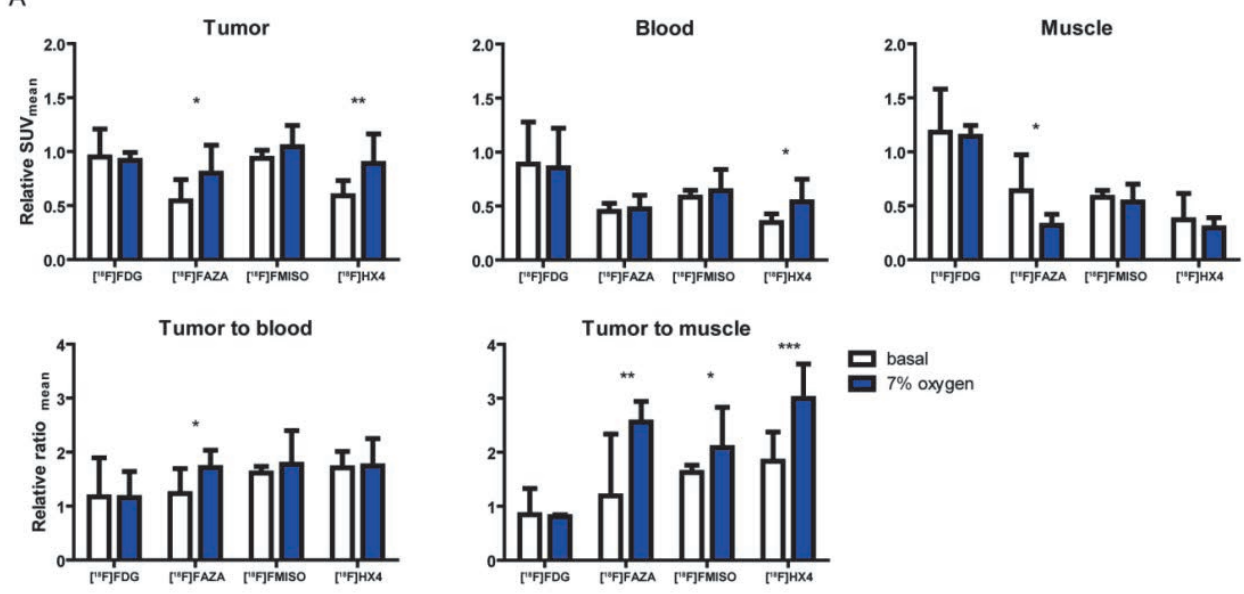

B
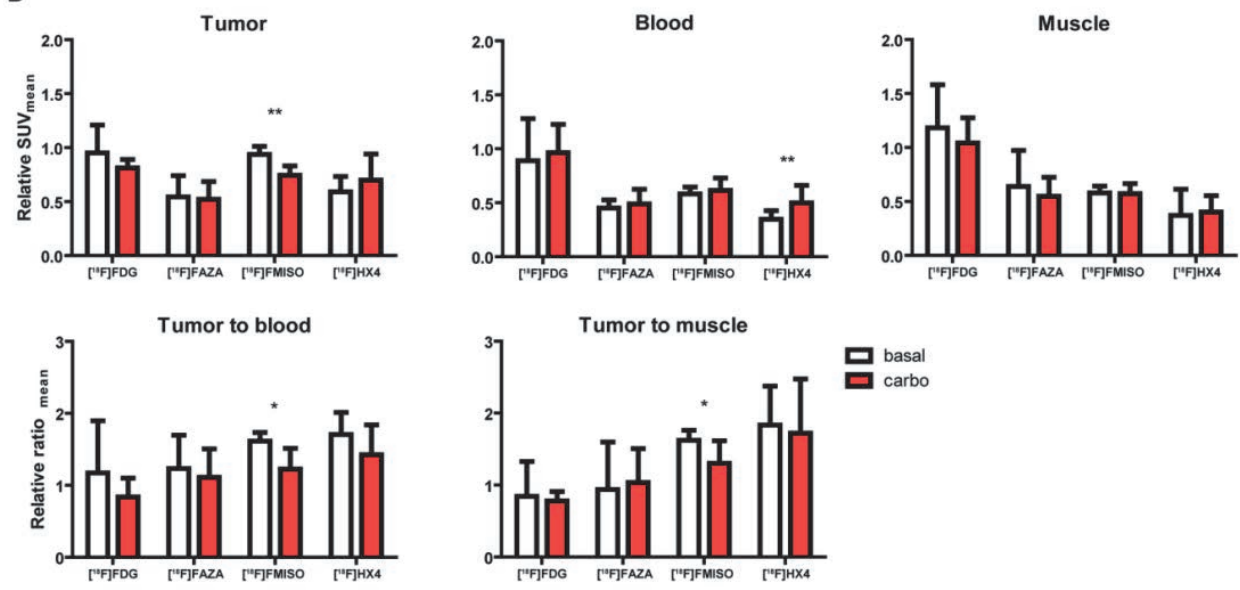

Figure 3.4: Oxygen modification. Relative $S U V_{\text {mean }}$ and tracer ratios compare untreated rats (basal) with those breathing $7 \%$ oxgyen (7\% oxygen) A) and those receiving nicotinamide/carbogen (carbo) treatmetn B). SUVS were calculated and compared 5 hours p.i. to 2 hours p.i. (vertical axis: relative SUV) for each organ separately and for the relative ratios TBR or TMR at 5 hours p.i. to 2 hours $p . i .{ }^{*} P<0.05,{ }^{*} P<0.01,{ }^{* * *} P<0.001$. Data are means \pm SD for basal: $\left[{ }^{18} \mathrm{~F}\right] F D G n=12,\left[{ }^{18} \mathrm{~F}\right]$ FAZA $n=14,\left[{ }^{18} \mathrm{~F}\right] \mathrm{FMISO} n=16$ and $\left[{ }^{18} \mathrm{~F}\right] \mathrm{HX} 4 \mathrm{n}=18$, for $7 \%$ oxygen: $\left[{ }^{18} \mathrm{~F}\right] \mathrm{FDG}$ $n=7,\left[{ }^{18} \mathrm{~F}\right]$ FAZA $n=7,\left[{ }^{18} \mathrm{~F}\right] \mathrm{FMISO} n=8$ and $\left[{ }^{18} \mathrm{~F}\right] \mathrm{HX} 4 \mathrm{n}=8$, for carbo: $\left[{ }^{18} \mathrm{~F}\right] \mathrm{FDG} n=8,\left[{ }^{18} \mathrm{~F}\right] \mathrm{FAZA} n=8,\left[{ }^{18} \mathrm{~F}\right] \mathrm{FMISO} n=6$ and $\left[{ }^{18} \mathrm{~F}\right] H X 4 n=14$. SUV $V_{\max }=$ maximum standard uptake value; TBR=tumor-to-blood ratio; TMR tumor to muscle ratio 
Rats were exposed to $7 \%$ oxygen breathing (Figure 3.1B), mimicking acute hypoxia. The relative $S_{U} V_{\text {mean }}$ tracer uptake after $7 \%$ oxygen treatment was significantly increased for $\left[{ }^{18} \mathrm{~F}\right] \mathrm{HX} 4(\mathrm{P}<0.01)$ and $\left[{ }^{18} \mathrm{~F}\right] \mathrm{FAZA}(\mathrm{P}<0.05)$ in the tumor, compared to that in the untreated animals (Figure 3.4A). The mean TBR for $\left[{ }^{18} \mathrm{~F}\right]$ FAZA was significantly increased $(P<0.05)$; the mean TMR showed a significant increase for all 3 hypoxia tracers $\left(\left[{ }^{18} \mathrm{~F}\right] \mathrm{FAZA} P<0.01\right.$, $\left[{ }^{18} \mathrm{~F}\right] \mathrm{FMISO} \mathrm{P}<0.05$ and $\left[{ }^{18} \mathrm{~F}\right] \mathrm{HX} 4 \mathrm{P}<0.01$ ) (Figure 3.4A). When the effect of maximal tumor uptake on increased hypoxia was studied, no significant effects were observed, although there was a trend towards increased uptake of $\left[{ }^{18} \mathrm{~F}\right] \mathrm{HX} 4$ in the tumor (Figure S3.2A).

The reversibility of tracer uptake on tumor reoxygenation was examined by treating the

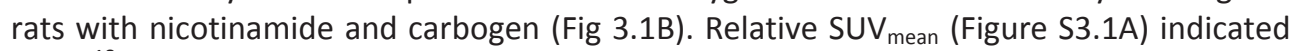
that $\left[{ }^{18} \mathrm{~F}\right] \mathrm{FMISO}$ remained stable over time in the baseline situation, whereas for other tracers decreased. Influencing tumors towards a more oxygenated state only caused a decrease in $\left[{ }^{18} \mathrm{~F}\right] \mathrm{FMISO}$ tumor uptake and did not change uptake of $\left[{ }^{18} \mathrm{~F}\right] \mathrm{HX} 4$ and $\left[{ }^{18} \mathrm{~F}\right] \mathrm{FAZA}$ (Fig 3.4B). Blood uptake values increased for $\left[{ }^{18} \mathrm{~F}\right] \mathrm{HX} 4$, but uptake in the muscle was not influenced. Mean TBR and TMR significantly decreased for $\left[{ }^{18} \mathrm{~F}\right] \mathrm{FMISO}$ and maximal values demonstrated comparable results (Figure S3.2B).

\section{DISCUSSION}

This study compares the frequently used hypoxia PET tracers $\left[{ }^{18} \mathrm{~F}\right] \mathrm{FMISO},\left[{ }^{18} \mathrm{~F}\right] \mathrm{FAZA}$, $\left[{ }^{18} \mathrm{~F}\right] \mathrm{HX} 4$ and the metabolic tracer $\left[{ }^{18} \mathrm{~F}\right] \mathrm{FDG}$ in an animal tumor model to assess their tumor-to-background ratios, spatial reproducibility and sensitivity to oxygen modification. The rat rhabdomyosarcoma R1 model with a large hypoxic fraction (18) was chosen to ensure sufficient visualization of the uptake of hypoxia tracers using a preclinical model on a clinical PET/CT scanner. Although some variation in tumor volume was seen, the hypoxic fraction was shown to be stable within this tumor model (18). PET acquisition was performed from the time of injection untill 6 hours p.i. to determine the optimal uptake in the tumor and highest TBR. In this study, we found the most optimal TBR for $\left[{ }^{18} \mathrm{~F}\right] \mathrm{FAZA}$ at 2 hours p.i. This finding is consistent with clinical studies in head and neck squamous cell carcinoma and non-small cell lung cancer (NSCLC) where imaging at 4 hours p.i. did not improve the TBR compared to 2 hours p.i. (19-21). $\left[{ }^{18} \mathrm{~F}\right] \mathrm{HX} 4$ shows an optimal TBR at 3 hours p.i which was also observed in an NSCLC patient study where image contrast did improve from 2 to 4 hours p.i. (22). As clinically demonstrated, $\left[{ }^{18} \mathrm{~F}\right] \mathrm{FMISO}$ does not show plateau formation, and has better TBR at later time points (23) which was also observed in this preclinical study. Comparative studies already have indicated that $\left[{ }^{18} \mathrm{~F}\right] \mathrm{HX} 4$ imaging in head and neck cancer patients at 1.5 hours p.i. was found to have TMR properties similar to those of $\left[{ }^{18} \mathrm{~F}\right.$ FMISO at 2 hours p.i. (24). This is also reflected in the biological half-life of the tracers, which is much higher in normal tissue for $\left[{ }^{18} \mathrm{~F}\right] \mathrm{FMISO}$ (clinical:12-13 hours (22), preclinical: 4.5 hours) than for $\left[{ }^{18} \mathrm{~F}\right] \mathrm{HX} 4$ (clinical: 4.3 hours (22), preclinical: 2.2 hours) or $\left[{ }^{18}\right.$ F]FAZA (preclinical: 2.8 hours). The findings from this preclinical study are in line with those from available clinical studies and although caution needs to be taken in extrapolation of the data, this might indicate that the results found here in this animal model can be translated to some extend to the clinical setting. 
One disparity between clinical and preclinical studies is the use of anesthetic drugs. In this study pentobarbital was used, and although it was shown that this causes a reduction in the radioactivity in blood and muscle, it did not influence tracer uptake in the tumor, nor did it lead to a significant change in tumor-to-background ratios (25).

The ultimate goal of tumor hypoxia imaging is to improve treatment outcome either by detecting hypoxia to aid in the decision to add specific anti-hypoxia drugs or by adapting radiation therapy using image guidance. Considering that hypoxia imaging can be used to generate personalized intensity modulated radiation therapy plans in which these radiation-resistant parts of the tumor can be boosted $(26,27)$, it would be desirable to have a tracer that shows stable uptake over time so that a single scan could be used for several days of treatment. Voxel-to-voxel analyses resulted in high reproducibility for all tracers within a 6-hour scan. Examining spatial reproducibility by comparing a high uptake region revealed good overlap between 2 consecutive scans $48 \mathrm{~h}$ apart for $\left[{ }^{18} \mathrm{~F}\right] \mathrm{FMISO},\left[{ }^{18} \mathrm{~F}\right] \mathrm{HX} 4$ and $\left[{ }^{18} \mathrm{~F}\right]$ FDG. For $\left[{ }^{18} \mathrm{~F}\right]$ FMISO this was also reported in a recent clinical head and neck patient study in which 2 scans were highly reproducible over 48 hours (28). However, voxel-to-voxel analysis of $\left[{ }^{18} \mathrm{~F}\right] \mathrm{FMISO}$ uptake over a 3-day interval found a correlation of the hypoxic distribution in less than $50 \%$ of the head and neck cancer patients (29). $\left[{ }^{18} \mathrm{~F}\right] \mathrm{FMISO}$ in the same patient population and during chemoradiotherapy showed a stable conformation of the hypoxic subvolumes (30). Our data show that reproducibility of $\left[{ }^{18} \mathrm{~F}\right]$ FAZA is poor after 48 hours, even without additional anticancer treatment. This is surprising given the fact that all investigated hypoxia tracers are based on the same nitroimidazole trapping mechanism. Contradictions in $\left[{ }^{18} \mathrm{~F}\right] \mathrm{FAZA}$ reproducibility are observed between different preclinical and clinical studies, which might also be caused by the differences in metabolism among organisms. Preclinical micro-PET analysis on $\left[{ }^{18} \mathrm{~F}\right]$ FAZA uptake showed voxel-to-voxel reproducibility between 2 baseline scans 24 hours apart; even after fractionated radiation therapy, a fairly stable intratumoral tracer distribution was observed (31). However, in a clinical trial, $\left[{ }^{18} \mathrm{~F}\right] \mathrm{FAZA}$ uptake was evaluated after several rounds of radiation therapy treatment and hypoxic regions were found not to be in the same location (19). Although $\left[{ }^{18} \mathrm{~F}\right] \mathrm{HX} 4$ shows good reproducibility in first clinical experiments (22), the stability of $\left[{ }^{18} \mathrm{~F}\right] \mathrm{HX} 4$ in detecting the hypoxic fraction during therapy needs to be further assessed. Uptake of $\left[{ }^{18} \mathrm{~F}\right] \mathrm{FDG}$ was clearly distinguishable from background and was highly reproducible, demonstrating the outstanding application of $\left[{ }^{18} \mathrm{~F}\right]$ FDG in the detection of tumors. However, we consider $\left[{ }^{18} \mathrm{~F}\right] \mathrm{FDG}$ to be a metabolic tracer rather that a marker for hypoxia.

Because tumor hypoxia is a dynamic process that consists of both chronic and acute hypoxia, a tumor's oxygen status changes continuously, and most hypoxia tracers mainly detect the chronic hypoxic fraction. However, there are suggestions that acute hypoxia also plays a prominent role in determining the treatment outcome (32). Changing a tumor's oxygen status by clamping or reduced oxygen breathing mimics this dynamic process and gives the opportunity to study the behavior of tracer uptake under these conditions. In the ideal situation, one would wish a hypoxia tracer to rapidly and specifically accumulate in the hypoxic regions with fast clearance in the nonhypoxic tissues. Binding of the tracer would be irreversible, and no circulating free available tracer would be present. 
Previous studies have shown that a treatment combining nicotinamide and carbogen increases a tumor's oxygen status $(9,33)$, whereas $7 \%$ oxygen breathing increases the hypoxic fraction (9). In this study, the oxygen modification was applied only 2.5 hours after tracer injection. Increasing the hypoxic fraction during tracer accumulation is dependent on the presence of unbound, circulating tracer. For all hypoxia tracers, circulating tracer was present after 3 hours, based on the measured activity in the blood (SUV of 2.4 for $\left[{ }^{18} \mathrm{~F}\right]$ FDG; 0.3 for $\left[{ }^{18} \mathrm{~F}\right] \mathrm{FAZA} ; 0.8$ for $\left[{ }^{18} \mathrm{~F}\right] \mathrm{FMISO}$, and 0.3 for $\left.\left[{ }^{18} \mathrm{~F}\right] \mathrm{HX} 4\right)$ however only $\left[{ }^{18} \mathrm{~F}\right] \mathrm{FAZA}$ and $\left[{ }^{18} \mathrm{~F}\right] \mathrm{HX} 4$ showed increased uptake in the tumor after $7 \%$ oxygen breathing. This effect was observed mainly in the mean values rather than in the maximum values, indicating that the tumor's overall oxygenation was altered, whereas the maximum value is determined by the severe hypoxic regions that will be less affected by this treatment. Exposure to high oxygen concentrations at 2.5 hours after tracer injection would prevent further accumulation or reverse tracer binding. For $\left[{ }^{18} \mathrm{~F}\right] \mathrm{FAZA}$, preclinical data are available that show reduced uptake after pure oxygen or carbogen breathing in tumor bearing mice $(8,34)$. In our experimental setting only $\left[{ }^{18} \mathrm{~F}\right] \mathrm{FMISO}$ showed a lower uptake upon reduced hypoxia. Together with the results of constant accumulation of $\left[{ }^{18} \mathrm{~F}\right] \mathrm{FMISO}$ in the tumor over time these data suggest that further accumulation is prevented when reducing the hypoxic fraction. Previous studies observed that $\left[{ }^{18} \mathrm{~F}\right] \mathrm{FMISO}$ uptake in squamous cell carcinoma bearing mice was influenced by the altered breathing condition (35). These experiments challenged the tracers to their limits and tried to mimic the changing oxygen concentrations in a tumor. It must be kept in mind that these results are influenced by tumor and animal model chosen and that the tracer metabolism is different in patients. Furthermore, exposing animals to modified oxygen concentration will introduce changes to the whole organism that might influence the distribution and metabolism of the tracer. Our data suggest that $\left[{ }^{18} \mathrm{~F}\right] \mathrm{HX} 4$ and $\left[{ }^{18} \mathrm{~F}\right] \mathrm{FAZA}$ are more sensitive to acute hypoxia while $\left[{ }^{18} \mathrm{~F}\right] \mathrm{FMISO}$ uptake is influenced by reoxygenation.

\section{CONCLUSIONS}

In conclusion, all investigated tracers showed different characteristics. The ultimate hypoxia tracer has not been developed, but this and other studies show that hypoxia imaging using the existing tracers gives extra information that can be very useful in the treatment of cancer patients. 


\section{ACKNOWLEDGEMENTS}

We acknowledge financial support from the Center for Translational Molecular Medicine framework 030-103 (AIR FORCE), European Union seventh framework programmes (METOXIA grant agreement no: 222741 and ARTFORCE grant agreement no: 257144) and the Kankeronderzoekfonds Limburg from the Health Foundation Limburg.

\section{CONFLICTS OF INTEREST}

The authors declare that they have no conflict of interest. 


\section{SUPPLEMENTARY DATA}

\section{Supplementary Materials and Methods}

Images were acquired using a clinical PET/CT scanner (Siemens Biograph 40, Siemens Healthcare) with an axial field of view (FoV) of $162 \mathrm{~mm}$, a transaxial FoV of $605 \mathrm{~mm}$ and a spatial resolution of $5.3 \mathrm{~mm}$ FWHM at the center of the FoV. The PET data were attenuation corrected using the acquired CT images. Also, correction for scatter (3D), randoms, dead time and decay of injected radionuclides was applied. First a topogram was acquired followed by a whole body CT scan using a $1 \mathrm{~mm}$ reconstructed slice thickness and a pitch of 0.8. For PET imaging an emission scan in list mode (LM) in one bed position was started simultaneously with tracer injection. LM data were rebinned using Fourier rebinning, and PET images were reconstructed for dynamic 60 minute imaging as $8 \times 15 \mathrm{sec}, 4 \times 30 \mathrm{sec}, 2 \times 1$ minute, $2 \times 2$ minutes and $10 \times 5$ minutes. The reconstructed voxel size was $3.04 \mathrm{~mm}$ in all dimensions. Every hour a similar scan was performed till 6 hours p.i. using a 20 minute emission scan, reconstructed as $4 \times 5$ minutes.

Reconstructed PET/CT images were analyzed in dedicated software (TrueD VC60; Siemens). Manual delineation of the volume of interest (VOI) was performed on the combined PET/CT image to obtain activity values $(\mathrm{Bq} / \mathrm{ml})$ for the tumor, muscle of the hind leg and blood pool (heart). After correcting the activity data for decay, data were quantified by calculating the SUV (activity in VOI / (injected dose/weight of animal)). SUV max $_{\text {indicates }}$ the averaged maximum SUV inside the VOI, SUV mean is the averaged mean activity in the VOI. Tumor-to-blood ratios (TBR) and tumor-to-muscle (TMR) ratios were calculated (SUV tumor / SUV blood or muscle, respectively) using either mean or maximal values for both vOls.

Non-rigid registration of the tumor was performed using in house developed registration software (36). The tumor contours were non-rigidly registered using an intensity based registration method (Morphons, 10 iterations, 8 resolution scales). A weighted sum accumulation of the deformation field was performed with a Gaussian regularization filter of 1.5 times the voxel size. The resulting deformation field was applied to the CT and the PET image of the rat. Next, the $50 \%$ of the total tumor volume with the highest SUV was defined, and the overlapping volume was calculated between the two scans. This was repeated on $40,30,20$ and $10 \%$ of the total tumor volume. 
A
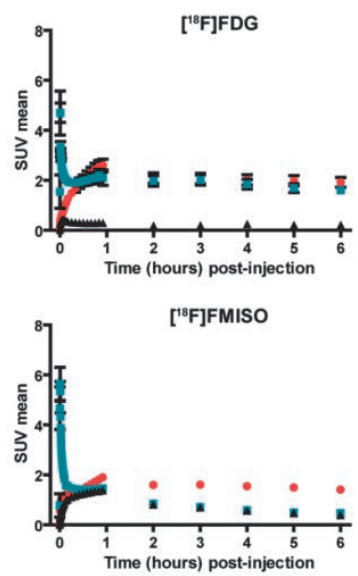

B

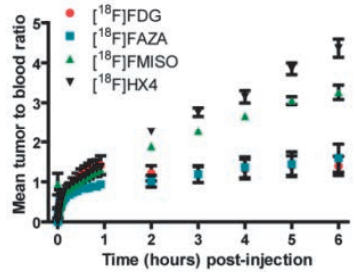

C

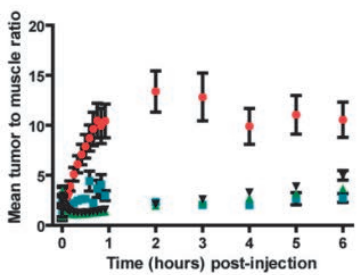

D

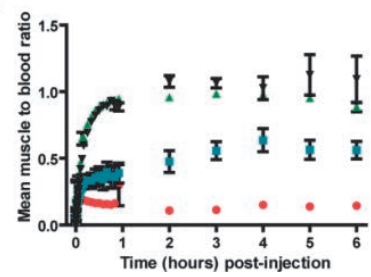

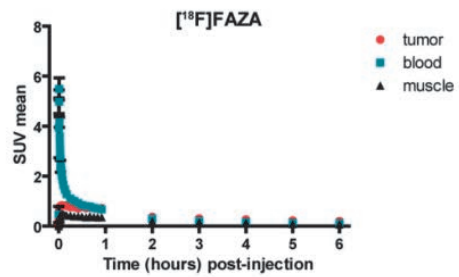

$\left[{ }^{18} \mathrm{~F}\right] \mathrm{HX} 4$
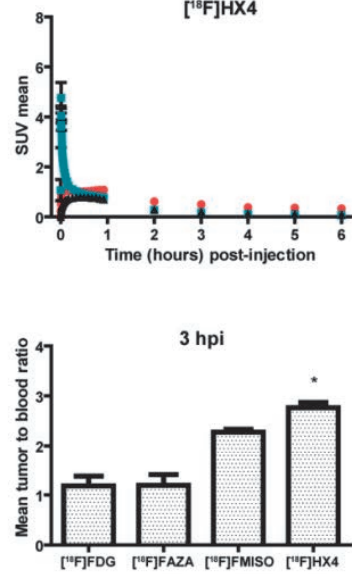

3hpi
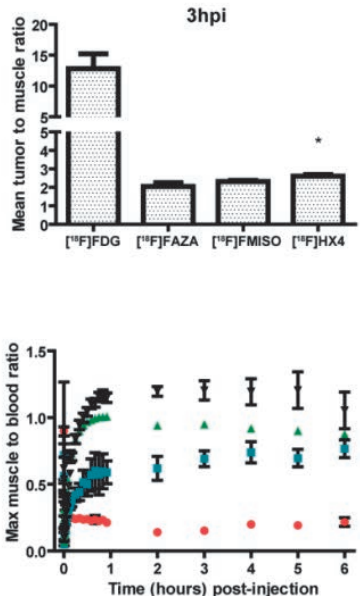

Figure S3.1: Mean SUV and tumor-to-background ratios. A) SUV mean of the tumor (red), blood (blue) and muscle (black) over time. ${ }^{18}$ F]FDG $n=12,\left[{ }^{18}\right.$ FJFAZA $n=13,\left[{ }^{18}\right.$ FJFMISO $n=16$ and $\left[{ }^{18} \mathrm{~F}\right] H X 4 n=18$ B) Mean TBR over time for $\left[{ }^{18}\right.$ FJFDG (red), $\left[{ }^{18}\right.$ FJFAZA (blue), $\left[{ }^{18}\right.$ FJFMISO (green) and $\left[{ }^{18} \mathrm{~F}\right] \mathrm{HX} 4$ (black) with TBR tracer comparison at three hours post-injection. $\left.{ }^{18} \mathrm{~F}\right] \mathrm{HX} 4$ had significant better TBR compared with the other tracers $\left[{ }^{18} \mathrm{~F}\right] F D G n=12,\left[{ }^{18} \mathrm{~F}\right] F A Z A$ $n=13,\left[^{18} \mathrm{~F}\right] \mathrm{FMISO} n=16$ and $\left[{ }^{18} \mathrm{~F}\right] \mathrm{HX} 4 \mathrm{n}=18 \mathrm{C}$ ) Mean tumor-to-muscle ratio over time. On the right side the mean TMR comparison of the four tracers at 3 hours p.i.: Mean TMR for $\left[{ }^{18} \mathrm{~F}\right] \mathrm{HX} 4$ was significantly higher than for the other hypoxia tracers: ${ }^{*} P_{\text {FAZA vs }} H_{X}=0.0114$ and ${ }^{*} P_{\text {FMIISO vs HXA }}=0.0102\left[{ }^{18}\right.$ F]FDG $n=12,\left[{ }^{18}\right.$ FJFAZA $n=14,\left[{ }^{18}\right.$ F]FMISO $n=16$ and $\left[{ }^{18} F\right] H X 4$ n=18. D) Mean and maximal muscle-to-blood ratio (MBR) over time. ${ }^{*} P<0.05$. $\left.{ }^{18} F\right] F D G n=12$, $\left[{ }^{18} \mathrm{~F}\right] F A Z A n=14,\left[{ }^{18} \mathrm{~F}\right] F M I S O n=16$ and $\left[{ }^{18} \mathrm{~F}\right] H X 4 n=18$. Data are means \pm SEM. 
A
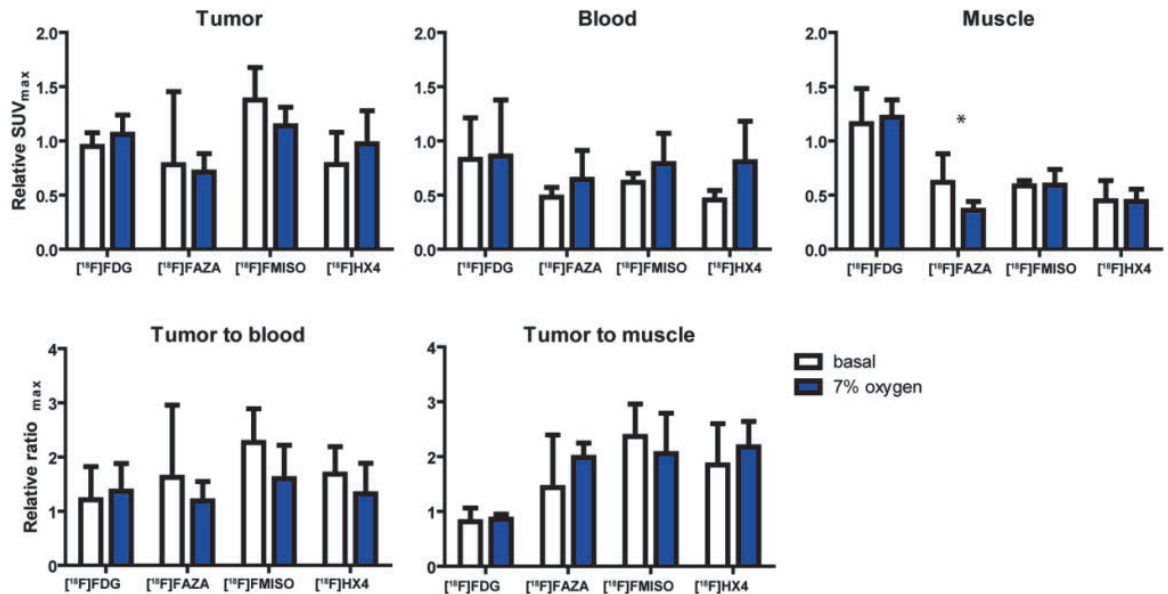

basal

$7 \%$ oxygen

B
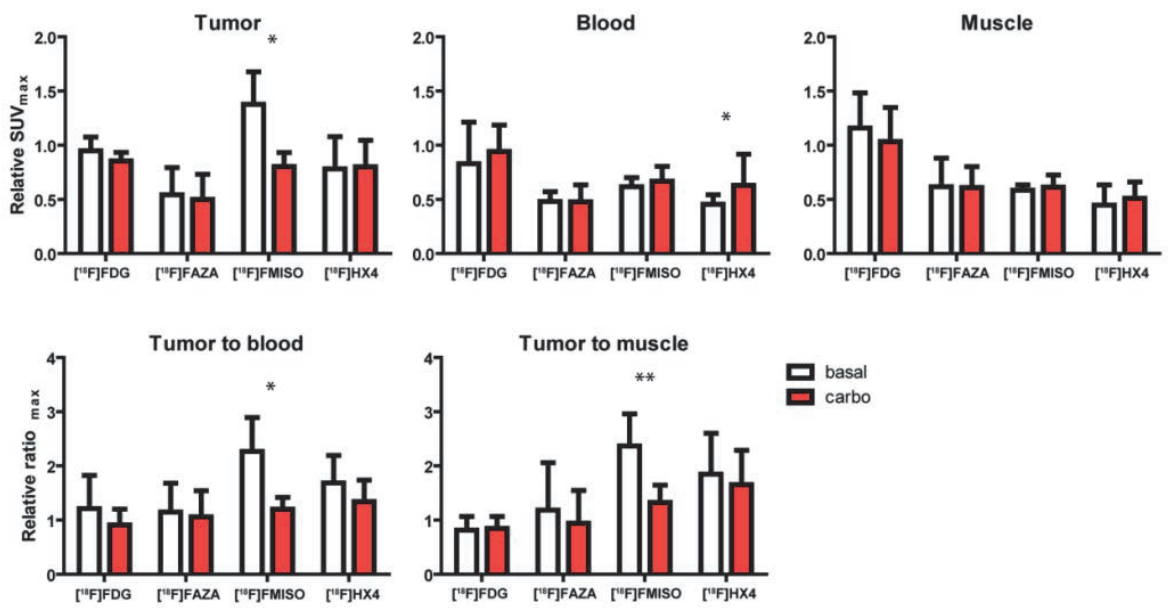

Figure S3.2: Oxygen modification. Relative SUV $\max$ or tracer ratios comparing untreated rats (basal) with A) $7 \%$ oxygen breathing (7\% oxygen) or B) nicotinamide/carbogen (carbo) treated rats. SUV's are calculated and represented as 5 hours p.i. relative to 2 hours p.i. for each organ separately or for the calculated TBR or TMR. ${ }^{*} P<0.05,{ }^{*} P<0.01$. Data are means $\pm S D$ for basal: $\left[{ }^{18} \mathrm{~F}\right] \mathrm{FDG} n=12,\left[{ }^{18} \mathrm{~F}\right] \mathrm{FAZA} n=14,\left[{ }^{18} \mathrm{~F}\right] \mathrm{FMISO} n=16$ and $\left[{ }^{18} \mathrm{~F}\right] \mathrm{HX} 4$ $n=18$, for 7\% oxygen: $\left[{ }^{18} \mathrm{~F}\right] F D G n=7,\left[{ }^{18} \mathrm{~F}\right]$ FAZA $n=7,\left[{ }^{18} \mathrm{~F}\right] \mathrm{FMISO} n=8$ and $\left[{ }^{18} \mathrm{~F}\right] \mathrm{HX} 4 \mathrm{n}=8$, for carbo: $\left[{ }^{18} \mathrm{~F}\right] \mathrm{FDG} n=8$, $\left[{ }^{18}\right.$ F]FAZA $n=8,\left[{ }^{18}\right.$ F]FMISO $n=6$ and $\left[{ }^{18} \mathrm{~F}\right] H X 4 n=14$. 


\section{REFERENCES}

1. Hammond EM, Asselin MC, Forster D, O'Connor JP, Senra JM, Williams KJ. The meaning, measurement and modification of hypoxia in the laboratory and the clinic. Clin Oncol (R Coll Radiol). 2014;26:277-88.

2. Horsman MR, Mortensen LS, Petersen JB, Busk M, Overgaard J. Imaging hypoxia to improve radiotherapy outcome. Nat Rev Clin Oncol. 2012;9:674-87.

3. Brown JM, Giaccia AJ. The unique physiology of solid tumors: opportunities (and problems) for cancer therapy. Cancer Res. 1998;58:1408-16.

4. Krohn KA, Link JM, Mason RP. Molecular imaging of hypoxia. J Nucl Med. 2008;49 Suppl 2:129S-48S.

5. Dubois L, Landuyt W, Cloetens L, Bol A, Bormans G, Haustermans K, et al. [18F]EF3 is not superior to [18F]FMISO for PET-based hypoxia evaluation as measured in a rat rhabdomyosarcoma tumour model. Eur J Nucl Med Mol Imaging. 2009;36:209-18.

6. Gronroos T, Bentzen L, Marjamaki P, Murata R, Horsman MR, Keiding S, et al. Comparison of the biodistribution of two hypoxia markers [18F]FETNIM and [18F]FMISO in an experimental mammary carcinoma. Eur J Nucl Med Mol Imaging. 2004;31:513-20.

7. Mahy P, De Bast M, de Groot T, Cheguillaume A, Gillart J, Haustermans K, et al. Comparative pharmacokinetics, biodistribution, metabolism and hypoxia-dependent uptake of [18F]-EF3 and [18F]-MISO in rodent tumor models. Radiother Oncol. 2008;89:353-60.

8. Reischl G, Dorow DS, Cullinane C, Katsifis A, Roselt P, Binns D, et al. Imaging of tumor hypoxia with [124I]IAZA in comparison with [18F]FMISO and [18F]FAZA--first small animal PET results. J Pharm Pharm Sci. 2007;10:203-11.

9. Dubois LJ, Lieuwes NG, Janssen MH, Peeters WJ, Windhorst AD, Walsh JC, et al. Preclinical evaluation and validation of [18F]HX4, a promising hypoxia marker for PET imaging. Proc Natl Acad Sci U S A. 2011;108:14620-5.

10. Carlin S, Zhang H, Reese M, Ramos NN, Chen Q, Ricketts SA. A comparison of the imaging characteristics and microregional distribution of 4 hypoxia PET tracers. Journal of nuclear medicine: official publication, Society of Nuclear Medicine. 2014;55:515-21.

11. Eschmann SM, Paulsen F, Reimold M, Dittmann H, Welz S, Reischl G, et al. Prognostic impact of hypoxia imaging with 18F-misonidazole PET in non-small cell lung cancer and head and neck cancer before radiotherapy. J Nucl Med. 2005;46:253-60.

12. Mortensen LS, Johansen J, Kallehauge J, Primdahl H, Busk M, Lassen P, et al. FAZA $\mathrm{PET} / \mathrm{CT}$ hypoxia imaging in patients with squamous cell carcinoma of the head and neck treated with radiotherapy: results from the DAHANCA 24 trial. Radiother Oncol. 2012;105:14-20.

13. van Loon J, Janssen $M H$, Ollers $M$, Aerts HJ, Dubois L, Hochstenbag $M$, et al. PET imaging of hypoxia using [18F]HX4: a phase I trial. Eur J Nucl Med Mol Imaging. 2010;37:1663-8.

14. Doss M, Zhang JJ, Belanger MJ, Stubbs JB, Hostetler ED, Alpaugh K, et al. Biodistribution and radiation dosimetry of the hypoxia marker $18 \mathrm{~F}-\mathrm{HX} 4$ in monkeys 
and humans determined by using whole-body PET/CT. Nucl Med Commun. 2010; 31:1016-24.

15. Lim JL, Berridge MS. An efficient radiosynthesis of [18F]fluoromisonidazole. Appl Radiat Isot. 1993;44:1085-91.

16. Reischl G, Ehrlichmann W, Bieg C, Solbach C, Kumar P, Wiebe LI, et al. Preparation of the hypoxia imaging PET tracer [18F]FAZA: reaction parameters and automation. Appl Radiat Isot. 2005;62:897-901.

17. Walsh JC, Kolb HC. Applications of click chemistry in radiopharmaceutical development. Chimia (Aarau). 2010;64:29-33.

18. Dubois L, Landuyt W, Haustermans K, Dupont P, Bormans G, Vermaelen P, et al. Evaluation of hypoxia in an experimental rat tumour model by [(18)F]fluoromisonidazole PET and immunohistochemistry. Br J Cancer. 2004;91:1947-54.

19. Servagi-Vernat S, Differding S, Hanin FX, Labar D, Bol A, Lee JA, et al. A prospective clinical study of F-FAZA PET-CT hypoxia imaging in head and neck squamous cell carcinoma before and during radiation therapy. European journal of nuclear medicine and molecular imaging. 2014.

20. Souvatzoglou M, Grosu AL, Roper B, Krause BJ, Beck R, Reischl G, et al. Tumour hypoxia imaging with [18F]FAZA PET in head and neck cancer patients: a pilot study. European journal of nuclear medicine and molecular imaging. 2007;34:1566-75.

21. Trinkaus ME, Blum R, Rischin D, Callahan J, Bressel M, Segard T, et al. Imaging of hypoxia with 18F-FAZA PET in patients with locally advanced non-small cell lung cancer treated with definitive chemoradiotherapy. J Med Imaging Radiat Oncol. 2013;57:475-81.

22. Zegers $C M$, van Elmpt W, Wierts R, Reymen B, Sharifi H, Ollers MC, et al. Hypoxia imaging with [(1)(8)F]HX4 PET in NSCLC patients: defining optimal imaging parameters. Radiotherapy and oncology : journal of the European Society for Therapeutic Radiology and Oncology. 2013;109:58-64.

23. Abolmaali N, Haase R, Koch A, Zips D, Steinbach J, Baumann M, et al. Two or four hour [(1)(8)F]FMISO-PET in HNSCC. When is the contrast best? Nuklearmedizin. 2011;50:22-7.

24. Chen L, Zhang Z, Kolb HC, Walsh JC, Zhang J, Guan Y. (1)(8)F-HX4 hypoxia imaging with PET/CT in head and neck cancer: a comparison with (1)(8)F-FMISO. Nucl Med Commun. 2012;33:1096-102.

25. Christian N, Bol A, De Bast M, Labar D, Lee J, Mahy P, et al. Determination of tumour hypoxia with the PET tracer [18F]EF3: improvement of the tumour-to-background ratio in a mouse tumour model. Eur J Nucl Med Mol Imaging. 2007;34:1348-54.

26. Hendrickson K, Phillips M, Smith W, Peterson L, Krohn K, Rajendran J. Hypoxia imaging with [F-18] FMISO-PET in head and neck cancer: potential for guiding intensity modulated radiation therapy in overcoming hypoxia-induced treatment resistance. Radiother Oncol. 2011;101:369-75.

27. Thorwarth D, Eschmann SM, Paulsen F, Alber M. Hypoxia dose painting by numbers: a planning study. Int J Radiat Oncol Biol Phys. 2007;68:291-300.

28. Okamoto S, Shiga T, Yasuda K, Ito YM, Magota K, Kasai K, et al. High reproducibility of tumor hypoxia evaluated by $18 \mathrm{~F}$-fluoromisonidazole PET for head and neck cancer. J Nucl Med. 2013;54:201-7. 
29. Nehmeh SA, Lee NY, Schroder H, Squire O, Zanzonico PB, Erdi YE, et al. Reproducibility of intratumor distribution of (18)F-fluoromisonidazole in head and neck cancer. Int J Radiat Oncol Biol Phys. 2008;70:235-42.

30. Bittner MI, Wiedenmann N, Bucher S, Hentschel M, Mix M, Weber WA, et al. Exploratory geographical analysis of hypoxic subvolumes using 18F-MISO-PET imaging in patients with head and neck cancer in the course of primary chemoradiotherapy. Radiother Oncol. 2013;108:511-6.

31. Busk M, Mortensen LS, Nordsmark M, Overgaard J, Jakobsen S, Hansen KV, et al. PET hypoxia imaging with FAZA: reproducibility at baseline and during fractionated radiotherapy in tumour-bearing mice. Eur J Nucl Med Mol Imaging. 2013;40:186-97.

32. Brown M. Henry S. Kaplan Distinguished Scientist Award Lecture 2007. The remarkable yin and yang of tumour hypoxia. Int J Radiat Biol. 2010;86:907-17.

33. Horsman MR, Overgaard J. Preclinical studies on how to deal with patient intolerance to nicotinamide and carbogen. Radiother Oncol. 2004;70:301-9.

34. Piert M, Machulla HJ, Picchio M, Reischl G, Ziegler S, Kumar P, et al. Hypoxia-specific tumor imaging with 18F-fluoroazomycin arabinoside. J Nucl Med. 2005;46:106-13.

35. Matsumoto K, Szajek L, Krishna MC, Cook JA, Seidel J, Grimes K, et al. The influence of tumor oxygenation on hypoxia imaging in murine squamous cell carcinoma using [64Cu]Cu-ATSM or [18F]Fluoromisonidazole positron emission tomography. Int J Oncol. 2007;30:873-81.

36. Janssens G, de Xivry JO, Fekkes S, Dekker A, Macq B, Lambin P, et al. Evaluation of nonrigid registration models for interfraction dose accumulation in radiotherapy. Med Phys. 2009;36:4268-76. 



\section{CHAPTER}

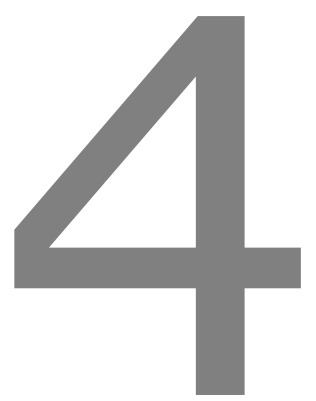

Repeatability of hypoxia PET imaging using $\left[{ }^{18} \mathrm{~F}\right] \mathrm{HX} 4$ in lung and head and neck cancer patients: a prospective multicenter trial

Catharina M.L. Zegers, Wouter van Elmpt, Katrin Szardenings, Hartmuth Kolb, Alan Waxman, Rathan M. Subramaniam, Dae Hyuk Moon, Jacqueline C. Brunetti, Shyam M. Srinivas, Philippe Lambin, David Chien 


\section{ABSTRACT}

Purpose: Hypoxia is an important parameter influencing tumor progression and treatment efficacy. The aim of this study was to investigate the repeatability of hypoxia PET imaging with $\left[{ }^{18} \mathrm{~F}\right] \mathrm{HX} 4$ in patients with head and neck and lung cancer.

Methods: Nine patients with lung cancer and ten with head and neck cancer were included in the analysis (NCT01075399). Two sequential pretreatment $\left[{ }^{18} \mathrm{~F}\right] \mathrm{HX} 4 \mathrm{PET} / \mathrm{CT}$ scans were acquired within 1 week. The maximal and mean standardized uptake values $\left(S_{U} V_{\max }\right.$ and $S_{U} V_{\text {mean }}$ ) were defined and the tumor-to-background ratios (TBR) were calculated. In addition, hypoxic volumes were determined as the volume of the tumor with a TBR $>1.2$ $\left(\mathrm{HV}_{1.2}\right)$. Bland Altman analysis of the uptake parameters was performed and coefficients of repeatability were calculated. To evaluate the spatial repeatability of the uptake, the $\mathrm{PET} / \mathrm{CT}$ images were registered and a voxel-wise comparison of the uptake was performed, providing a correlation coefficient.

Results: All parameters of $\left[{ }^{18} \mathrm{~F}\right] \mathrm{HX} 4$ uptake were significantly correlated between scans: $\operatorname{SUV}_{\text {max }}(r=0.958, p<0.001), \operatorname{SUV}_{\text {mean }}(r=0.946, p<0.001), \operatorname{TBR}_{\text {max }}(r=0.962, p<0.001)$ and $\mathrm{HV}_{1.2}$ $(r=0.995, p<0.001)$. The relative coefficients of repeatability were $15 \%\left(S U V_{\text {mean }}\right), 17 \%$ $\left(\mathrm{SUV}_{\max }\right)$ and $17 \%\left(\mathrm{TBR}_{\max }\right)$. Voxel-wise analysis of the spatial uptake pattern within the tumors provided an average correlation of $0.65 \pm 0.14$.

Conclusions: Repeated hypoxia PET scans with $\left[{ }^{18} \mathrm{~F}\right] \mathrm{HX} 4$ provide reproducible and spatially stable results in patients with head and neck cancer and patients with lung cancer. $\left[{ }^{18} \mathrm{~F}\right] \mathrm{HX} 4$ PET imaging can be used to assess the hypoxic status of tumors and has the potential to aid hypoxia targeted treatments. 


\section{INTRODUCTION}

$\left[{ }^{18} \mathrm{~F}\right] \mathrm{HX} 4$ is a new 2-nitroimidazole PET imaging agent for hypoxia, in which structureactivity relationships have been used to optimize pharmacokinetic and clearance properties $(1,2)$. Tumor hypoxia is a condition where insufficiently vascularized tumor cells deprived of oxygen not only become more aggressive and malignant, but also more resistant to treatment by radiation and chemotherapy (3-5). The presence of hypoxia is therefore generally considered a poor prognostic disease marker in cancer patients (6). However, it is difficult to measure oxygen levels reproducibly and non-invasively in a highly heterogeneous tumor environment. Reliable diagnostic methods to detect and quantify tumor hypoxia are therefore needed. It has been hypothesized and currently being investigated that inclusion of hypoxic cell sensitizers during treatment, i.e., the delivery of higher radiotherapy doses to hypoxic regions (7) or the use of hypoxiatargeting therapy (8-11), might improve the outcome in patients with hypoxic tumors (12). $\left[{ }^{18} \mathrm{~F}\right] \mathrm{HX} 4$ has the potential to serve as a clinically useful diagnostic tool to aid the application of hypoxia targeting therapies to those patients who will most likely benefit from them $(13,14)$.

This pilot phase 2 study was primarily designed as a test-retest study to investigate the repeatability of $\left[{ }^{18} \mathrm{~F}\right] \mathrm{HX} 4$ as a non-invasive PET imaging marker for detection of tumor hypoxia regions. Here we present the results in patients with lung cancer and patients with head and neck (H\&N) cancer.

\section{MATERIALS AND METHODS}

\section{Patients}

This multicentered study (NCT01075399) was conducted in accordance with the ethical principles of Good Clinical Practice, according to the International Conference on Harmonization of Technical Requirements for Registration of Pharmaceuticals for Human Use (ICH). Both the FDA and the institutional review boards of the participating institutions approved the study protocol and the informed consent form. All participants reviewed and signed the informed consent form before study entry. $\left[{ }^{18} \mathrm{~F}\right] \mathrm{HX} 4 \mathrm{PET} / \mathrm{CT}$ images were acquired of 19 patients: 9 with lung cancer and 10 with H\&Ncancer. The patients underwent two sequential pre-treatment $\left[{ }^{18} \mathrm{~F}\right] \mathrm{HX} 4$ PET/CT scans within 1 week to assess repeatability. Patient characteristics are presented in Table 4.1. 
Table 4.1: Patient characteristics.

\begin{tabular}{|c|c|c|c|c|c|}
\hline Patient ID & Gender & Lesion Location & TNM & Pathology & $\operatorname{GTV}\left(\mathrm{cm}^{3}\right)$ \\
\hline \multicolumn{6}{|c|}{ Lung Cancer } \\
\hline 01 & $\mathrm{~F}$ & RUL lung & T4N2M1 & Adeno & 88.8 \\
\hline 02 & $M$ & LUL lung & T4N3M1 & Small cell & 361.5 \\
\hline 03 & $\mathrm{M}$ & $\mathrm{R}$ precarina & T1N3M0 & Large cell & 5.2 \\
\hline 04 & $\mathrm{~F}$ & $\mathrm{R}$ mediastinum & T3N2MO & Large cell & 251.6 \\
\hline 05 & $\mathrm{~F}$ & RLL lung & $\mathrm{T} 2 \mathrm{~N} 2 \mathrm{MO}$ & Adeno & 87.6 \\
\hline 06 & $\mathrm{M}$ & RUL lung & T4N3M0 & Squamous cell & 23.0 \\
\hline 07 & $\mathrm{M}$ & RUL lung & T2aN2M1 & Adeno & 10.2 \\
\hline 08 & $\mathrm{~F}$ & RUL lung & T2NOMO & Adeno & 9.2 \\
\hline 09 & $\mathrm{~F}$ & LUL lung & T1bNOMO & Large cell & 4.1 \\
\hline \multicolumn{6}{|c|}{ Head and Neck Cancer } \\
\hline 10 & $M$ & R Neck LN & T1N1M0 & NA & 20.5 \\
\hline 11 & $\mathrm{~F}$ & Anterior larynx & T3N2cM0 & Squamous cell & 7.0 \\
\hline 12 & $\mathrm{~F}$ & L Soft palate & T4NOMO & Squamous cell & 79.9 \\
\hline 13 & $M$ & $\mathrm{R}$ Base of tongue & T2N2aMo & Squamous cell & 2.6 \\
\hline 14 & $M$ & R neck & $\mathrm{T} 2 \mathrm{~N} 2 \mathrm{bMO}$ & Squamous cell & 17.8 \\
\hline 15 & $M$ & L Aryepiglottic fold & T2N2aMo & Squamous cell & 31.6 \\
\hline 16 & $M$ & R Piriform sinus & T1N1M0 & Squamous cell & 6.9 \\
\hline 17 & $\mathrm{~F}$ & R Maxillary sinus & T4N2M0 & Adenoid cystic & 248.1 \\
\hline 18 & $M$ & R Base of tongue & T1N2bM0 & Squamous cell & 5.3 \\
\hline 19 & $M$ & R Sinonasal Space & T4aNOMO & Undifferentiated & 68.3 \\
\hline
\end{tabular}

$\mathrm{RUL}=$ right upper lobe, $\mathrm{LUL}=$ left upper lobe, $\mathrm{RLL}$ = right lower lobe, $\mathrm{R}=$ right, $\mathrm{L}=$ left, $\mathrm{LN}=$ lymph node

\section{Radiochemistry}

$\left[{ }^{18} \mathrm{~F}\right] \mathrm{HX} 4$ (flortanidazole, 3-[18F]fluoro-2-(4-((2-nitro-1H-imidazol-1-yl)methyl )-1H-1,2,3triazol-1-yl)-propan-1-ol) was prepared by Siemens Molecular Imaging (Culver City, CA) or a Siemens PETNET qualified manufacturing site and delivered to each site on the day of injection. The radiosynthesis has been described previously (15). Briefly, the precursor (Siemens Molecular Imaging Inc., Culver City, California, USA) was reacted with 18F$\mathrm{K} 2.2 .2$, and $\mathrm{K}_{2} \mathrm{CO}_{3}$ in $\mathrm{MeCN}$ at $110^{\circ} \mathrm{C}$ for $10 \mathrm{~min}$, followed by a deprotection step using 1.0 $\mathrm{mol} / \mathrm{I} \mathrm{HCl}$ at $100^{\circ} \mathrm{C}$ for $5 \mathrm{~min}$. $\left[{ }^{18} \mathrm{~F}\right] \mathrm{HX} 4$ was purified by RP-HPLC and stabilized with ascorbic acid before sterile filtration. In order to be released, each dose of $\left[{ }^{18} \mathrm{~F}\right] \mathrm{HX} 4$ had to have a radiochemical purity greater than $95 \%$. 


\section{Scanners and technical parameters}

$\left[{ }^{18} \mathrm{~F}\right] \mathrm{HX} 4 \mathrm{PET} / \mathrm{CT}$ scans were performed using high-resolution full-ring PET/CT scanners, including a GE Discovery, GE Discovery LS, Philips Gemini, and a Siemens Biograph PET/CT scanner. Images were reconstructed using scanner-specific parameters in accordance to each facility's standard procedure, including at least attenuation and scatter correction. Repeat scans were performed on the same PET/CT scanner, using the same protocol and patient positioning without respiratory gating.

\section{$\left[{ }^{18} \mathrm{~F}\right] \mathrm{HX} 4 \mathrm{PET} / \mathrm{CT}$ imaging}

For each $\left[{ }^{18} \mathrm{~F}\right] \mathrm{HX} 4 \mathrm{PET} / \mathrm{CT}$ scan, the patient received a single intravenous bolus injection of $368 \pm 48 \mathrm{MBq}$ (range: $199-488 \mathrm{MBq}$ ) of $\left[{ }^{18} \mathrm{~F}\right] \mathrm{HX} 4$, followed by a saline flush. A static $\mathrm{PET} / \mathrm{CT}$ scan was acquired with an acquisition time of 3 minutes (range 1.7 - $5 \mathrm{~min}$ ) per bed position, after an uptake time of $99 \pm 10$ minutes (range: $89-125 \mathrm{~min}$ ). The average difference in uptake time between repeat PET scans was $6 \pm 7$ min (range: $0-27 \mathrm{~min}$ ).

\section{Image evaluation of $\left[{ }^{18} \mathrm{~F}\right] \mathrm{HX} 4$}

$\left[{ }^{18} \mathrm{~F}\right] \mathrm{HX} 4$ PET/CT scans were analyzed using an Inveon Research Workplace (Edition 4.0.0.3, Siemens, Germany). Gross tumor volumes (GTV) of the primary lesion or largest lymph node were defined in centimeters cubed by manual contouring the tumor on the CT images by one observer (D.C.). These tumor delineations were applied to the PET images and the maximal and mean standardized uptake values $\left(S_{U} U V_{m a x}, S U V_{\text {mean }}\right)$ were measured in grams per milliliter. Under the assumption of water density, the SUV is reported as unitless. For each patient, the reference tissue was defined by contouring a volume of interest (VOI; sphere with a radius of $25 \mathrm{~mm}$ ) in a large (thigh) muscle on the CT image. From this muscle VOI the $\mathrm{SUV}_{\text {mean }}(\mathrm{M})$ was determined.

Tumor to background ratios (TBR) were calculated by dividing Tumor SUV $\mathrm{V}_{\text {max }}$ and $\mathrm{SUV}_{\text {mean }}$ by Muscle SUV mean $(\mathrm{M})$

$$
\begin{aligned}
& \mathrm{TBR}_{\text {max }}=\text { Tumor SUV }_{\text {max }} / \mathrm{M} \\
& \mathrm{TBR}_{\text {mean }}=\text { Tumor SUV } \\
& \text { mean }
\end{aligned}
$$

The hypoxic volume ( $\mathrm{HV}$; in centimeters cubed) of each tumor was defined as the $\left[{ }^{18} \mathrm{~F}\right] \mathrm{HX} 4$ tumor volume with a TBR $>1.2\left(\mathrm{HV}_{1.2}\right)$ or $\mathrm{TBR}>1.4\left(\mathrm{HV}_{1.4}\right)$ :

$\mathrm{HV}_{1.2}=$ Volume within GTV with TBR $>1.2$

$\mathrm{HV}_{1.4}=$ Volume within GTV with $\mathrm{TBR}>1.4$ 
The fraction of hypoxic volume (FHV, percent) of each tumor was determined by dividing the HV by its respective GTV:

$$
\begin{aligned}
& \mathrm{FHV}_{1.2}=\mathrm{HV}_{1.2} / \mathrm{GTV} \\
& \mathrm{FHV}_{1.4}=\mathrm{HV}_{1.4} / \mathrm{GTV}
\end{aligned}
$$

To evaluate the repeatability of the heterogeneous uptake pattern, the second $\left[{ }^{18} \mathrm{~F}\right] \mathrm{HX} 4$ $\mathrm{PET} / \mathrm{CT}$ scans were rigidly registered, inspected for accurate registration and a voxel-wise comparison of the SUV values within the GTV was performed.

\section{Statistics}

For all parameters, the mean $\pm S D$ are reported. The relationships among GTV-based parameters $\left(\mathrm{SUV}_{\text {mean }}, \mathrm{SUV}_{\max }, \mathrm{TBR}, \mathrm{HV}, \mathrm{FHV}\right.$ ) extracted from repeat $\left[{ }^{18} \mathrm{~F}\right] \mathrm{HX} 4 \mathrm{PET}$ images were analyzed by calculating Pearson correlation coefficients. A $p$ value $<0.05$ was assumed to be statistically significant. In addition, a Bland Altman analysis was performed for all parameters providing the mean difference of each parameter and the absolute and relative coefficients of repeatability (CR: 1.96x SD), defined as the value below which the difference between two measurement will be within $95 \%$ probability. To evaluate the voxel-wise analysis a linear fit of the data was performed, providing the correlation coefficient and slope. A Bland Altman plot was created providing the difference in uptake for each matching voxel ( $\triangle S U V$ ) with its lower and upper limits of agreement of the $95 \%$ confidence interval. In addition a histogram of SUVs within the GTV was prepared.

\section{RESULTS}

$\left[{ }^{18} \mathrm{~F}\right] \mathrm{HX} 4 \mathrm{PET} / \mathrm{CT}$ imaging in nine patients with lung cancer patients and ten with $\mathrm{H} \& \mathrm{~N}$ cancer were included in the analysis. Two sequential baseline $\left[{ }^{18} \mathrm{~F}\right] \mathrm{HX} 4 \mathrm{PET} / \mathrm{CT}$ scans were performed at an average interval of 1.1 days (range $=1-2$ days) in patients with lung cancer and 2.1 days (range $=1-6$ days) in patients with $\mathrm{H} \& \mathrm{~N}$ cancer.

\section{$\left[{ }^{18} \mathrm{~F}\right] \mathrm{HX} 4$ uptake in the GTV}

$\left[{ }^{18} \mathrm{~F}\right] \mathrm{HX} 4$ uptake on the first PET scan varied considerably among tumors on both the first scan with an average SUV max $_{\text {m }} 1.86 \pm 0.52$ (range $=1.2-2.9$ ) and SUV mean $_{\text {of }} 1.20 \pm 0.28$ (range $=0.85-1.90)$ and the second scan with an average SUV $\max$ of $1.84 \pm 0.50$ (range = $1.15-2.82$ ) and SUV $_{\text {mean }}$ of $1.20 \pm 0.28$ (range $=0.92-1.97$; Table 4.2). 
Table 4.2: Repeatability of the $\left[{ }^{18} \mathrm{~F}\right] \mathrm{HX} 4$ uptake and hypoxic tumor volume (HV) and fraction (FHV) using a threshold of 1.2 times background

\begin{tabular}{|c|c|c|c|c|c|c|c|c|c|c|}
\hline \multirow[b]{2}{*}{ ID } & \multicolumn{2}{|c|}{ SUV $_{\text {mean }}$} & \multicolumn{2}{|l|}{ SUV $_{\text {max }}$} & \multicolumn{2}{|l|}{$\mathrm{TBR}_{\max }$} & \multicolumn{2}{|c|}{$\mathrm{HV}_{1.2}\left[\mathrm{~cm}^{3}\right]$} & \multicolumn{2}{|c|}{$\mathrm{FHV}_{1.2}[\%]$} \\
\hline & Scan1 & Scan2 & Scan1 & Scan2 & Scan1 & Scan2 & Scan1 & Scan2 & Scan1 & Scan2 \\
\hline \multicolumn{11}{|c|}{ Lung Cancer } \\
\hline 01 & 1.20 & 1.09 & 2.15 & 2.21 & 1.72 & 1.87 & 14.01 & 11.15 & 15.78 & 11.33 \\
\hline 02 & 1.38 & 1.49 & 2.87 & 2.74 & 2.40 & 2.10 & 147.30 & 148.30 & 40.76 & 38.55 \\
\hline 03 & 1.17 & 1.19 & 1.47 & 1.60 & 1.20 & 1.27 & 0.06 & 0.26 & 1.23 & 9.30 \\
\hline 04 & 1.90 & 1.97 & 2.93 & 2.82 & 2.03 & 1.94 & 177.60 & 203.30 & 70.62 & 74.95 \\
\hline 05 & 1.16 & 1.10 & 1.68 & 1.67 & 1.50 & 1.66 & 18.82 & 18.50 & 21.48 & 36.04 \\
\hline 06 & 0.89 & 0.90 & 1.46 & 1.55 & 1.49 & 1.62 & 2.49 & 1.80 & 10.85 & 9.52 \\
\hline 07 & 0.94 & 1.03 & 1.57 & 1.64 & 1.47 & 1.51 & 0.45 & 1.45 & 4.41 & 9.67 \\
\hline 08 & 1.36 & 1.51 & 1.98 & 2.25 & 1.64 & 1.75 & 3.58 & 4.58 & 38.85 & 47.92 \\
\hline 09 & 1.19 & 1.07 & 1.63 & 1.47 & 1.17 & 1.16 & 0.00 & 0.00 & 0.00 & 0.00 \\
\hline Mean \pm & $1.24 \pm$ & $1.26 \pm$ & $1.97 \pm$ & $1.99 \pm$ & $1.63 \pm$ & $1.65 \pm$ & $40.5 \pm$ & $43.3 \pm$ & $22.6 \pm$ & $26.4 \pm$ \\
\hline SD & 0.29 & 0.33 & 0.57 & 0.53 & 0.39 & 0.31 & 69.9 & 76.6 & 23.4 & 24.6 \\
\hline \multicolumn{11}{|c|}{ Head and Neck Cancer } \\
\hline 10 & 0.91 & 0.92 & 1.25 & 1.29 & 1.04 & 1.01 & 0.00 & 0.00 & 0.00 & 0.00 \\
\hline 11 & 0.89 & 0.99 & 1.23 & 1.31 & 1.04 & 1.08 & 0.00 & 0.00 & 0.00 & 0.00 \\
\hline 12 & 1.85 & 1.72 & 2.52 & 2.39 & 1.32 & 1.27 & 3.90 & 1.54 & 4.89 & 2.02 \\
\hline 13 & 1.09 & 0.97 & 1.30 & 1.15 & 1.17 & 1.09 & 0.00 & 0.00 & 0.00 & 0.00 \\
\hline 14 & 1.15 & 0.99 & 1.71 & 1.37 & 1.42 & 1.28 & 0.90 & 0.13 & 5.04 & 0.67 \\
\hline 15 & 1.17 & 1.12 & 1.72 & 1.80 & 1.44 & 1.69 & 3.65 & 5.57 & 11.56 & 20.57 \\
\hline 16 & 0.98 & 1.05 & 1.77 & 1.96 & 1.79 & 2.08 & 1.01 & 2.03 & 14.55 & 33.83 \\
\hline 17 & 1.23 & 1.17 & 2.48 & 2.33 & 3.35 & 3.15 & 211.20 & 203.70 & 85.14 & 80.03 \\
\hline 18 & 1.15 & 1.18 & 1.49 & 1.39 & 1.36 & 1.23 & 0.60 & 0.27 & 11.33 & 5.11 \\
\hline 19 & 1.26 & 1.28 & 2.12 & 2.05 & 1.96 & 1.99 & 28.73 & 41.24 & 42.07 & 57.56 \\
\hline Mean \pm & $1.17 \pm$ & $1.14 \pm$ & $1.76 \pm$ & $1.70 \pm$ & $1.59 \pm$ & $1.59 \pm$ & $25.0 \pm$ & $25.5 \pm$ & $17.5 \pm$ & $20.0 \pm$ \\
\hline SD & 0.27 & 0.23 & 0.48 & 0.46 & 0.69 & 0.67 & 66.0 & 63.9 & 26.9 & 28.5 \\
\hline Mean士 & $1.20 \pm$ & $1.20 \pm$ & $1.86 \pm$ & $1.84 \pm$ & $1.61 \pm$ & $1.62 \pm$ & $32.3 \pm$ & $33.9 \pm$ & $19.9 \pm$ & $23.0 \pm$ \\
\hline SD & 0.28 & 0.28 & 0.52 & 0.50 & 0.55 & 0.52 & 66.4 & 68.8 & 24.7 & 26.2 \\
\hline
\end{tabular}

The uptake parameters from the first and second scans were highly correlated: $r=0.958$ for $\operatorname{SUV}_{\max }\left(p<0.001\right.$, Figure 4.1) and $r=0.946$ for $\operatorname{SUV}_{\text {mean }}(, p<0.001$, Figure S4.1) High correlations between scans were also seen within each subgroup of cancer patients: $r=$ 0.972 for $\operatorname{SUV}_{\max }(p<0.001)$ and $r=0.960$ for $\operatorname{SUV}_{\text {mean }}(p<0.001)$ in those with lung cancer, and $r=0.945$ for $\operatorname{SUV}_{\max }(p<0.001)$ and $r=0.952$ for $\operatorname{SUV}_{\text {mean }}(p<0.001)$ in those with $\mathrm{H} \& \mathrm{~N}$ cancer. In the Bland Altman analysis, SUV $\mathrm{Sax}_{\text {, }}$ showed a mean difference of 0.02 with an absolute CR of 0.29 and a repeatability percentage of $17 \%$ (Figure 4.1 ) and SUV mean $_{\text {n }}$ showed a mean difference of 0.01 with an absolute CR of 0.18 and a repeatability percentage of $15 \%$. 
High correlations were also seen for $\operatorname{TBR}_{\max }(r=0.962, p<0.001$; Figure 4.1) and TBR $\mathrm{Tmean}(r$ $=0.965, p<0.001)$. High correlations were also seen within each subgroup of cancer patients: $r=0.939$ forTBR $\max (p<0.001)$ and $r=0.972$ for $\operatorname{TBR}_{\text {mean }}(p<0.001)$ in those with lung cancer, and similarly $r=0.972$ for $\operatorname{TBR}_{\max }(p<0.001)$ and $r=0.964$ for $\operatorname{TBR}_{\text {mean }}(p<$ 0.001 ) in those with $\mathrm{H} \& \mathrm{~N}$ cancer. In the Bland-Altman analysis, $T B R_{\max }$, showed a mean difference of -0.01 with an absolute CR of 0.30 and a repeatability percentage of $17 \%$ (Figure 4.1), and $\mathrm{TBR}_{\text {mean }}$ showed a mean difference of -0.01 with an absolute CR of 0.11 and a repeatability percentage of $10 \%$.
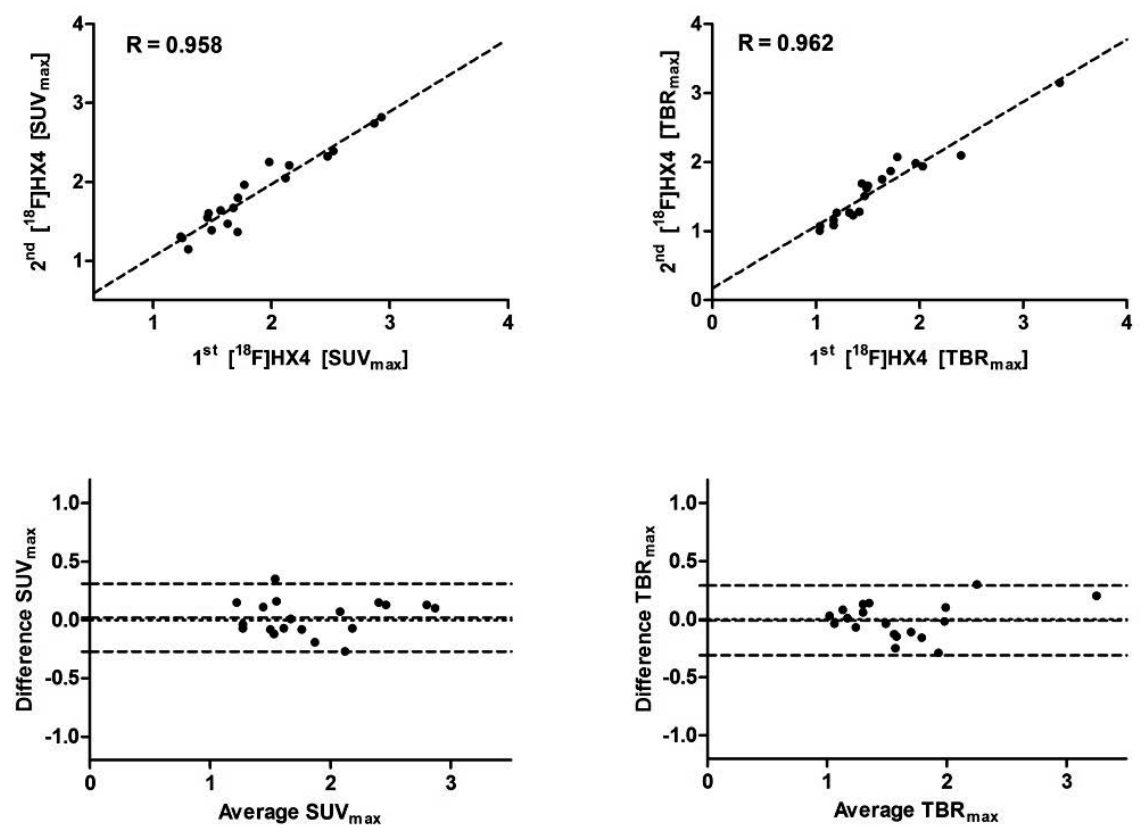

Figure 4.1: Correlation and Bland Altman plots (including the 95\% confidence interval) of the image parameters $S U V_{\max }$ and $T B R_{\max }$.

\section{HV and FHV analysis}

The average tumor volume was $70 \mathrm{~cm}^{3}$ (range $\left.=2.6-361 \mathrm{~cm}^{3}\right)$. The average $\mathrm{HV}_{1.2}$ in the first scan was $32 \mathrm{~cm}^{3}$ (range $=0-211 \mathrm{~cm}^{3}$ ) and in the second scan was $34 \mathrm{~cm}^{3}$ (range $=0$ $204 \mathrm{~cm}^{3}$; Table 4.2). For $\mathrm{HV}_{1.2}$, there was a high correlation between the first and second scans ( $r=0.995, p<0.001$, Figure S4.1) which was retained in each subgroup of cancer patients $r=0.997(p<0.001)$ in those with lung cancer and and $r=0.998(p<0.001)$ in those with $\mathrm{H} \& \mathrm{~N}$ cancer. In the Bland-Altman analysis, $\mathrm{HV}_{1.2}$ showed a mean difference of $1.55 \mathrm{~cm}^{3}$ with an absolute $\mathrm{CR}$ of $13.5 \mathrm{~cm}^{3}$ (Figure $\mathrm{S} 4.1$ ).

Applying the higher threshold of 1.4 times the background, in the first scan the average $\mathrm{HV}_{1.4}$ was $19 \mathrm{~cm}^{3}$ (range $=0-175 \mathrm{~cm}^{3}$ ) and in the second scan was $19 \mathrm{~cm}^{3}$ (range $=0-162$ 
$\mathrm{cm}^{3}$; Table S4.1). For $\mathrm{HV}_{1.4}$, there was also a consistently high correlation between the first and second scans $(r=0.982, p<0.001)$ which was retained in each subgroup of patients: $r$ $=0.959(p<0.001)$ in those with lung cancer. and $r=0.999(p<0.001)$ in those with H\&N cancer. In the Bland-Altman analysis, $\mathrm{HV}_{1,4}$ showed a mean difference of $0.08 \mathrm{~cm}^{3}$ with a confidence interval from -17.2 to $17.4 \mathrm{~cm}^{3}$.

There was a wide range of $\mathrm{FHV}_{1.2}$ due to varying levels of hypoxia among the tumors. In the first scan the average $\mathrm{FHV}_{1.2}$ was $20 \pm 25 \%$ (range $=0-85 \%$ ) and in the second scan the average $\mathrm{FHV}_{1.2}$ was $23 \pm 26 \%$ (range $=0-80 \%$; Table 4.2 ). This was also seen when the higher threshold of 1.4 times the background was applied: in the first scan the average FHV $_{1.4}$ was $9 \pm 18 \%$ (range $0-71 \%$ ) and in the second scan the average $\mathrm{FHV}_{1.4}$ was $10 \pm 17 \%$ (range: 0 - 63\%; Table S4.1).

For $\mathrm{FHV}_{1.2}$ there was a high correlation between the first and second scans $(r=0.957, \mathrm{p}<$ $0.001)$ whih was retained in each subgroup of cancer patients $r=0.966(p<0.001)$ in those withlung cancer and $r=0.950(p<0.001)$ in those with $\mathrm{H} \& \mathrm{~N}$ cancer. For $\mathrm{FHV}_{1.4}$ there was also a high correlation between the first and second scans $(r=0.975, p<0.001)$, which was retained in each subgroup of cancer patients $r=0.963(p<0.001)$ in those with lung cancer and $r=0.985(\mathrm{p}<0.001)$ in those with H\&N cancer. In the Bland-Altman analysis, $\mathrm{FHV}_{1.2}$ showed a mean difference of $-3.1 \%$ with an absolute $\mathrm{CR}$ of $14.9 \%$, and $\mathrm{FHV}_{1.4}$. showed a mean difference of $-0.9 \%$ and an absolute $\mathrm{CR}$ of $7.8 \%$.

Using 1.2 times the background as the threshold to determine FHV, 79\% of the tumors $(15 / 19)$ were found to have some level of hypoxia but when the higher threshold of 1.4 times the background was applied to determine the FHV, only $47 \%$ of the tumors $(9 / 19)$ were characterized as having tumor hypoxia.

\section{Repeatability of the spatial uptake pattern}

An example of voxel-wise image analysis in a patient with head and neck cancer (patient 12 ) is shown in Figure 4.2. Comparison of the heterogeneous uptake within the GTV between the first and second $\left[{ }^{18} \mathrm{~F}\right] \mathrm{HX} 4$ PET scan showed a moderate to strong correlation in the majority of patients, with an average correlation coefficient of $0.65 \pm 0.14$. There were two exceptions (Patient 14 and 16) in whom a poor correlation was observed $(R=$ 0.38 and 0.39). The average slope and intercept of the linear fit of the data were $0.56 \pm 0.17$ and $0.47 \pm 0.19$, respectively. The Bland-Altman analysis showed an average $\triangle$ SUV of $0.02 \pm 0.06$, with a lower and upper limit of agreement of $0.15 \pm 0.09$ and $0.19 \pm 0.08$. Examples of voxel-wise image analysis in patients with lung cancer (patient 1 and 4) are shown in Figure 4.3. In addition, the results for each patient are shown in Table 4.3. 
84 Chapter 4
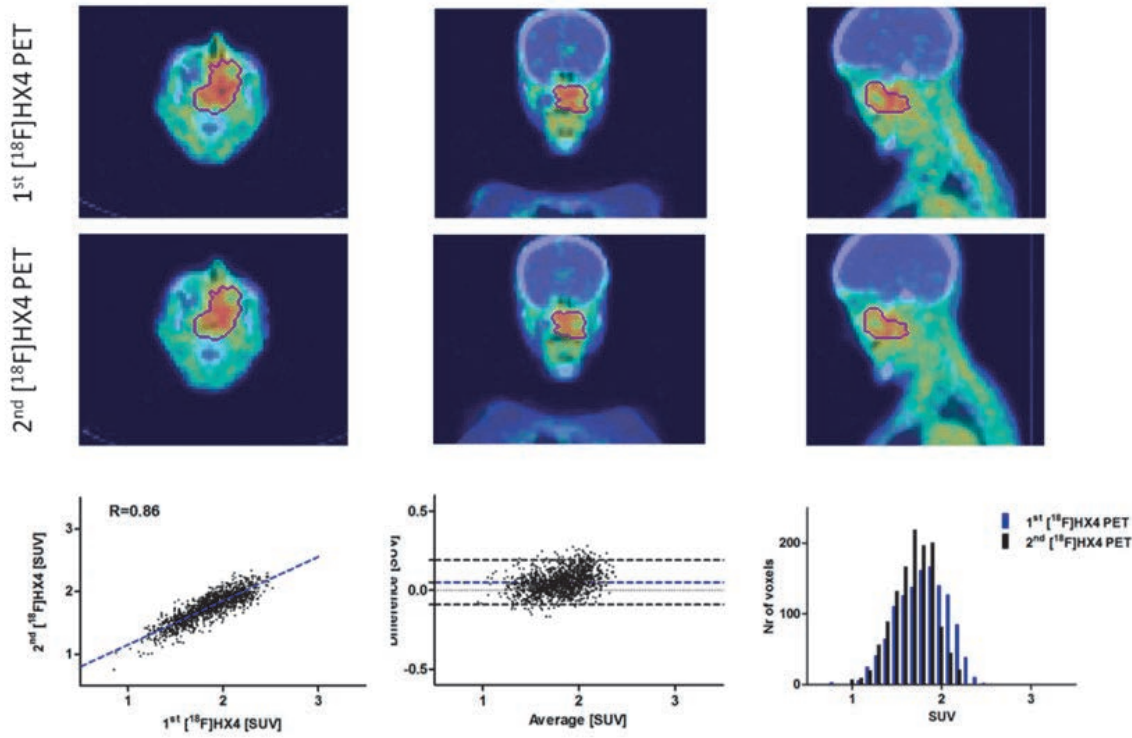

Figure 4.2: Example of voxel-wise analysis in a patient with head and neck cancer (patient 12). The axial, coronal, and sagittal planes of the first and rigidly registered second $\left.{ }^{18} \mathrm{~F}\right] H X 4$ PET/CT scan are shown. The gross tumor volume is delineated. The bottom row shows the correlation plot, the Bland-Altman, and the histogram plot of the voxels within the gross tumor volume. 
Table 4.3: Results of the voxel-wise analysis. Pearsons correlation coefficient (R), slope and intercept of the linear fit. Mean of the difference in SUV ( $\triangle S U V)$ of the Bland Altman plot with the 95\% Confidence Interval (CI), with the lower (LLA) and upper (ULA) limit of agreement.

\begin{tabular}{|c|c|c|c|c|c|c|}
\hline \multirow[b]{3}{*}{ ID } & \multicolumn{3}{|c|}{ Correlation plot } & \multicolumn{3}{|l|}{ Bland Altman } \\
\hline & \multirow[b]{2}{*}{$\mathbf{R}$} & \multirow[b]{2}{*}{ slope } & \multirow[b]{2}{*}{ intercept } & \multirow[b]{2}{*}{ Mean $\Delta$ SUV } & \multicolumn{2}{|l|}{$95 \% \mathrm{Cl}$} \\
\hline & & & & & LLA & ULA \\
\hline \multicolumn{7}{|c|}{ Lung Cancer } \\
\hline 01 & 0.51 & 0.39 & 0.64 & 0.05 & -0.21 & 0.30 \\
\hline 02 & 0.61 & 0.46 & 0.85 & -0.06 & -0.34 & 0.23 \\
\hline 03 & 0.69 & 0.72 & 0.35 & -0.01 & -0.15 & 0.13 \\
\hline 04 & 0.85 & 0.82 & 0.4 & -0.03 & -0.20 & 0.14 \\
\hline 05 & 0.58 & 0.78 & 0.02 & 0.12 & -0.10 & 0.35 \\
\hline 06 & 0.83 & 0.63 & 0.27 & 0.03 & -0.10 & 0.15 \\
\hline 07 & 0.55 & 0.45 & 0.62 & -0.05 & -0.24 & 0.13 \\
\hline 08 & 0.82 & 0.86 & 0.28 & -0.04 & -0.21 & 0.13 \\
\hline 09 & 0.62 & 0.44 & 0.52 & 0.07 & -0.07 & 0.21 \\
\hline Mean \pm & $0.67 \pm$ & $0.63 \pm$ & $0.42 \pm$ & $0.02 \pm$ & $-0.19 \pm$ & $0.22 \pm$ \\
\hline SD & 0.13 & 0.18 & 0.24 & 0.07 & 0.08 & 0.12 \\
\hline \multicolumn{7}{|c|}{ Head and Neck Cancer } \\
\hline 10 & 0.63 & 0.59 & 0.35 & 0.01 & -0.12 & 0.14 \\
\hline 11 & 0.84 & 0.71 & 0.31 & -0.02 & -0.12 & 0.07 \\
\hline 12 & 0.86 & 0.70 & 0.45 & 0.05 & -0.09 & 0.19 \\
\hline 13 & 0.69 & 0.30 & 0.45 & 0.15 & 0.06 & 0.25 \\
\hline 14 & 0.38 & 0.28 & 0.61 & 0.11 & -0.07 & 0.28 \\
\hline 15 & 0.56 & 0.49 & 0.52 & 0.04 & -0.12 & 0.20 \\
\hline 16 & 0.39 & 0.37 & 0.68 & -0.03 & -0.25 & 0.19 \\
\hline 17 & 0.63 & 0.61 & 0.42 & 0.03 & -0.23 & 0.30 \\
\hline 18 & 0.70 & 0.49 & 0.61 & -0.01 & -0.11 & 0.09 \\
\hline 19 & 0.63 & 0.49 & 0.64 & 0.00 & -0.21 & 0.21 \\
\hline Mean \pm & $0.63 \pm$ & $0.50 \pm$ & $0.50 \pm$ & $0.03 \pm$ & $-0.13 \pm$ & $0.19 \pm$ \\
\hline SD & 0.16 & 0.15 & 0.13 & 0.06 & 0.09 & 0.07 \\
\hline Mean \pm & $0.65 \pm$ & $0.56 \pm$ & $0.46 \pm$ & $0.03 \pm$ & $-0.16 \pm$ & $0.21 \pm$ \\
\hline SD & 0.14 & 0.17 & 0.19 & 0.06 & 0.09 & 0.10 \\
\hline
\end{tabular}




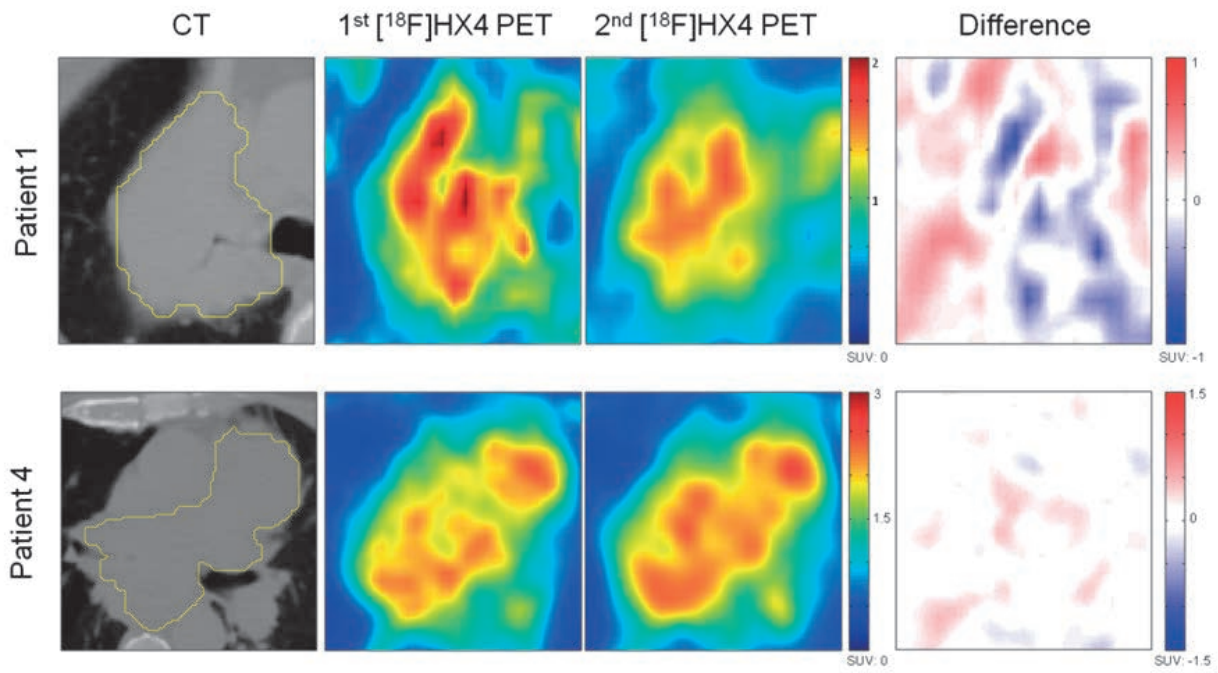

Figure 4.3: Examples of voxel-wise analysis in patients with lung cancer (patients 1 and 4). The axial plane of the $C T$ with the gross tumor volumes delineated in yellow, the first $\left[{ }^{18} \mathrm{~F}\right] H X 4$ PET scan, the rigidly registered second $\left[{ }^{18} \mathrm{~F}\right] \mathrm{HX} 4$ PET scan and the difference map of the two scans are shown.

\section{DISCUSSION}

The aim of this study was to investigate the repeatability of $\left[{ }^{18} \mathrm{~F}\right] \mathrm{HX} 4$ as a non-invasive PET imaging marker for the detection of tumor hypoxia in patients with lung cancer and patients with $\mathrm{H} \& \mathrm{~N}$ cancer. Tumor hypoxia is known to be a dynamic process characterized by the presence of acute and chronic hypoxia. Acute hypoxia is usually the result of a blockage or disruption in the perfusion of the tumor, while chronic hypoxia is mainly caused by limitations of oxygen diffusion due to an inefficient blood vessel network, which results in larger distances between the blood vessels and tumor tissue. Static PET imaging will show only the hypoxic status at one specific time-point and containinformation about both acute and chronic hypoxia. To be able to select patients for treatment with antihypoxia therapy and/or for a hypoxia based radiotherapy dose redistribution, it is important to gain an insight into the day-to-day variability in tumor hypoxia and its spatial location. Therefore we compared $\left[{ }^{18} \mathrm{~F}\right] \mathrm{HX} 4$ uptake, tumor-to-muscle levels and hypoxic fractions between two consecutive $\left[{ }^{18} \mathrm{~F}\right] \mathrm{HX} 4$ PET scans. To obtain information about the spatial distribution of tumor hypoxia, a voxel-wise comparison of the $\left[{ }^{18} \mathrm{~F}\right] \mathrm{HX} 4$ uptake was performed.

While there was, as anticipated, a large interpatient variability in $\left[{ }^{18} \mathrm{~F}\right] \mathrm{HX} 4$ uptake, no major differences were observed between patients with $\mathrm{H} \& \mathrm{~N}$ or patients with lung cancer. The average SUV of $\left[{ }^{18} \mathrm{~F}\right] \mathrm{HX} 4$ was identical for lung cancer $(1.2 \pm 0.3)$ and H\&N cancer lesions $(1.2 \pm 0.3)$. There is no standardized method to define tumor hypoxia on PET images. The threshold value for defining tumor hypoxia is dependent on the tracer, tracer pharmacokinetics, and other imaging parameters (16). In our previous study (16) we 
showed that a threshold of 1.2 times background at $2 \mathrm{~h}$ after injection provides a similar FHV and hypoxic lesion detection rate to imaging using a threshold of 1.4 times background at 4 hours after injection. In the current analysis we included both thresholds to quantify the HV. First we defined the threshold as an uptake above 1.2 times the background level. In this case $89 \%(8 / 9)$ of the lung and $70 \%(7 / 10)$ of the head and neck cancer patients had a hypoxic tumor volume. These percentages are in agreement with previously published results showing, for example, hypoxia in $72 \%$ of non-small-cell lung cancer patients (16) or $84 \%$ of those with H\&N cancer (17). Increasing the threshold to 1.4 times background level, results in decreases in the proportion of hypoxic lesions detected to $67 \%$ of lung cancer lesions (6/9) and $30 \%$ of $H \& N$ cancer lesions(3/10).

At the tumor-level we observed a high correlation for the frequently used parameters to quantify tumor hypoxia $\left(S_{U V} V_{\text {max }}, S U V_{\text {mean }}, T B R, H V\right.$ and FHV). This is in agreement with the results ofa study by Okamoto et al. (18), who evaluated the reproducibility of the hypoxia PET tracer $\left[{ }^{18} \mathrm{~F}\right]$ FMISO in patients with $\mathrm{H} \& \mathrm{~N}$ cancer. They found a high correlation for SUV $_{\max }$, TBR and HV. However, these results do not agreewith the previous results of Nehmeh et al. (19), who found a considerable variability in the intratumoral uptake between repeat $\left[{ }^{18} \mathrm{~F}\right.$ FMISO PET scans. The reproducibility of the hypoxia PET tracer $\left[{ }^{18}\right.$ F]FAZA was evaluated by Busk et al. $(20)$ in a mouse model and showed good reproducibility. In comparison to $\left[{ }^{18} \mathrm{~F}\right] \mathrm{FDG}$ PET/CT imaging, our observed repeatability percentages $\left(\mathrm{SUV}_{\max } 17 \%\right.$ and $\left.\mathrm{SUV}_{\text {mean }} 15 \%\right)$ are smaller than the relative differences required to exceed test-retest variability, which should be larger than $25 \%$ for SUV $_{\max }$ and $20 \%$ for SUV $_{\text {mean }}(21)$. Since $\left[{ }^{18} \mathrm{~F}\right] \mathrm{HX} 4$ has a lower uptake than $\left[{ }^{18} \mathrm{~F}\right] \mathrm{FDG}$, results from comparison of the two tracers should be interpreted with caution. However, comparing our relative coefficients of repeatability with the results of the low uptake $\left[{ }^{18} \mathrm{~F}\right] \mathrm{FDG}$ measurements (Figure $1 \mathrm{C}$ of de Langen et al. (21)), the observed $\left[{ }^{18} \mathrm{~F}\right] \mathrm{HX} 4$ repeatability percentage of the $\mathrm{SUV}_{\text {max }}(17 \%)$ is much lower than expected based on $\left[{ }^{18} \mathrm{~F}\right] \mathrm{FDG}$ (approximately $35 \%$ ). This high repeatability of $\left[{ }^{18} \mathrm{~F}\right] \mathrm{HX} 4$ PET imaging parameters at the tumor level provides confidence that hypoxia PET imaging using $\left[{ }^{18} \mathrm{~F}\right] \mathrm{HX} 4$ can be used to reliably detect and quantify tumor hypoxia. This is essential for the use of hypoxia PET imaging as a predictor of treatment response or for the monitoring of changes in hypoxia during treatment. The detection of hypoxia using $\left[{ }^{18} \mathrm{~F}\right] \mathrm{HX} 4 \mathrm{PET} / \mathrm{CT}$ at the tumor level could therefore be used to identify patients who might benefit from hypoxia-targeted treatment (22).

To evaluate the stability of the heterogeneous uptake pattern of $\left[{ }^{18} \mathrm{~F}\right] \mathrm{HX} 4$, a voxel-wise comparison was performed. This analysis showed reproducible results $(R>0.5)$ in the majority (17 out of 19) patients with lung cancer or H\&N cancer. The observed repeatability is in agreement with the previous publication of Peeters et al., showing a high repeatability of the $\left[{ }^{18} \mathrm{~F}\right] \mathrm{HX} 4$ uptake in a rat rhabdomyosarcoma model (23). Repeatability studies using the alternative hypoxia tracer $\left[{ }^{18} \mathrm{~F}\right] \mathrm{FMISO}$ reported contradictory results: Okamoto et al. (18) and Bittner et al. (24) found good repeatability, while Nehmeh et al. (19) observed variability in the spatial uptake. For the hypoxia tracer $\left[{ }^{18} \mathrm{~F}\right] \mathrm{FAZA}$, repeated PET/CT imaging was performed during the course of radiotherapy. While Mortensen et al. (25) found a stable location of the HV during treatment, Servagi-Vernat (26) found a spatial move of the HV. The spatial reproducibility of tumor hypoxia, as measured by a hypoxia PET tracer is essential for hypoxia PET-based radiotherapy planning. Three-dimensional- 
information of the hypoxic areas within the tumor can be used to tailor radiotherapy treatment to give a higher radiation dose to the hypoxic subvolumes (27). In this study, $\left[{ }^{18} \mathrm{~F}\right] \mathrm{HX} 4 \mathrm{PET} / \mathrm{CT}$ imaging was able to identify stable hypoxic areas in the majority of patients. Therefore, this imaging technique could potentially enable the reliable treatment of hypoxic areas with an increased radiotherapy dose. Several studies have already shown that it is feasible to perform radiotherapy dose planning based on hypoxia PET images (12, $28,29)$.

There are some limitations to this study. First, patients with very heterogeneous disease were included. These tumors have a different histology and might therefore express a different phenotype regarding acute versus chronic tumor hypoxia, which could possibly affect the reproducibility of tracer uptake. Nevertheless, even in this heterogeneous population, a high repeatability in $\left[{ }^{18} \mathrm{~F}\right] \mathrm{HX} 4 \mathrm{PET} / \mathrm{CT}$ uptake was observed. Second, the study design was multi-centric; therefore different PET/CT scanners were used with different physical characteristics and different acquisition protocols. Differences in resolution among the scanners might have led to differences in the tumor hypoxia detection rates. In general we expect with all scanners a partial volume effect, and particularly in small lesions with low uptake and with a small HV this would cause larger differences in absolute uptake measurements. Also, breathing motion in patients with the lung cancer could have caused blurring of the PET signal. The differences in acquisition protocol, i.e. acquisition time per bed position and uptake period, will lead to differences in the observed signal-to-noise ratios, and TBR and SUV measurements (30,31). Nevertheless, since we used each patient as his or her own control, the partial volume effect and the effect of different scanners should have had only a minor influence on the repeatability results. Third, the $\left[{ }^{18} \mathrm{~F}\right] \mathrm{HX} 4 \mathrm{PET}$ scans were on average, acquired at $99 \mathrm{~min}$ after injection, with a maximal difference in the time from injection toacquisition of 27 min. Studies reported after this study was completed have shown that the contrast between tumor and background increases up to 4 hafter injection. Therefore the image contrast might be suboptimal and differences in uptake parameters observed might have been due to the differences in the time from injection to acquisition(31).

In conclusion, repeated PET imaging with the hypoxia tracer $\left[{ }^{18} \mathrm{~F}\right] \mathrm{HX} 4$ provides reliable and reproducible results regarding the (spatial) uptake in patients with head and neck and lung cancer. $\left[{ }^{18} \mathrm{~F}\right] \mathrm{HX} 4$ has the potential to quantify hypoxia in tumors and aid hypoxia-targeted treatments.

\section{ACKNOWLEDGEMENTS}

Authors acknowledge financial support from the Dutch technology Foundation STW (grant $\mathrm{n}^{\circ} 10696$ DuCAT), which is the applied science division of NWO, and the Technology Programme of the Ministry of Economic Affairs. Authors also acknowledge financial support from EU 7th framework program (ARTFORCE - $\mathrm{n}^{\circ}$ 257144), Kankeronderzoekfonds Limburg from the Health Foundation Limburg and the Dutch Cancer Society (KWF UM 2011-5020, KWF MAC 2013-6425). 


\section{CONFLICTS OF INTEREST}

K. Szardenings is an employee at Threshold Pharmaceuticals, the company which owns and develops $\left[{ }^{18} \mathrm{~F}\right] \mathrm{HX} 4$. 
90 |Chapter 4

\section{SUPPLEMENTARY DATA}

Table S4.1: Repeatability of the $\left.{ }^{18} \mathrm{~F}\right] \mathrm{HX} 4$ hypoxic tumor volume(HV) and fraction (FHV) using a threshold of 1.4 times background

\begin{tabular}{|c|c|c|c|c|}
\hline \multirow[b]{2}{*}{ Patient ID } & \multicolumn{2}{|l|}{$\mathrm{HV}_{1.4}\left[\mathrm{~cm}^{3}\right]$} & \multicolumn{2}{|l|}{$\mathrm{FHV}_{1.4}[\%]$} \\
\hline & Scan 1 & Scan 2 & Scan 1 & Scan 2 \\
\hline \multicolumn{5}{|l|}{ Lung Cancer } \\
\hline 01 & 3.05 & 2.33 & 3.43 & 2.37 \\
\hline 02 & 77.43 & 54.67 & 21.42 & 14.21 \\
\hline 03 & 0.00 & 0.00 & 0.00 & 0.00 \\
\hline 04 & 96.00 & 121.34 & 38.16 & 44.73 \\
\hline 05 & 1.60 & 3.52 & 1.83 & 6.86 \\
\hline 06 & 0.45 & 0.25 & 1.95 & 1.32 \\
\hline 07 & 0.05 & 0.15 & 0.49 & 1.00 \\
\hline 08 & 1.39 & 1.79 & 15.11 & 18.75 \\
\hline 09 & 0.00 & 0.00 & 0.00 & 0.00 \\
\hline Mean $\pm S D$ (Lung) & $20.0 \pm 38.1$ & $20.5 \pm 41.8$ & $9.2 \pm 13.3$ & $9.9 \pm 14.7$ \\
\hline \multicolumn{5}{|c|}{ Head and Neck Cancer } \\
\hline 10 & 0.00 & 0.00 & 0.00 & 0.00 \\
\hline 11 & 0.00 & 0.00 & 0.00 & 0.00 \\
\hline 12 & 0.00 & 0.00 & 0.00 & 0.00 \\
\hline 13 & 0.00 & 0.00 & 0.00 & 0.00 \\
\hline 14 & 0.06 & 0.00 & 0.36 & 0.00 \\
\hline 15 & 0.13 & 1.41 & 0.41 & 5.20 \\
\hline 16 & 0.31 & 0.52 & 4.42 & 8.68 \\
\hline 17 & 175.37 & 161.63 & 70.68 & 63.50 \\
\hline 18 & 0.00 & 0.00 & 0.00 & 0.00 \\
\hline 19 & 12.06 & 18.74 & 17.66 & 26.15 \\
\hline Mean $\pm S D(H N)$ & $18.8 \pm 55.1$ & $18.2 \pm 50.7$ & $9.4 \pm 22.2$ & $10.2 \pm 17.4$ \\
\hline Mean $\pm S D$ (total) & $19.4 \pm 46.6$ & $19.3 \pm 45.4$ & $9.3 \pm 18.0$ & $10.4 \pm 20.4$ \\
\hline
\end{tabular}



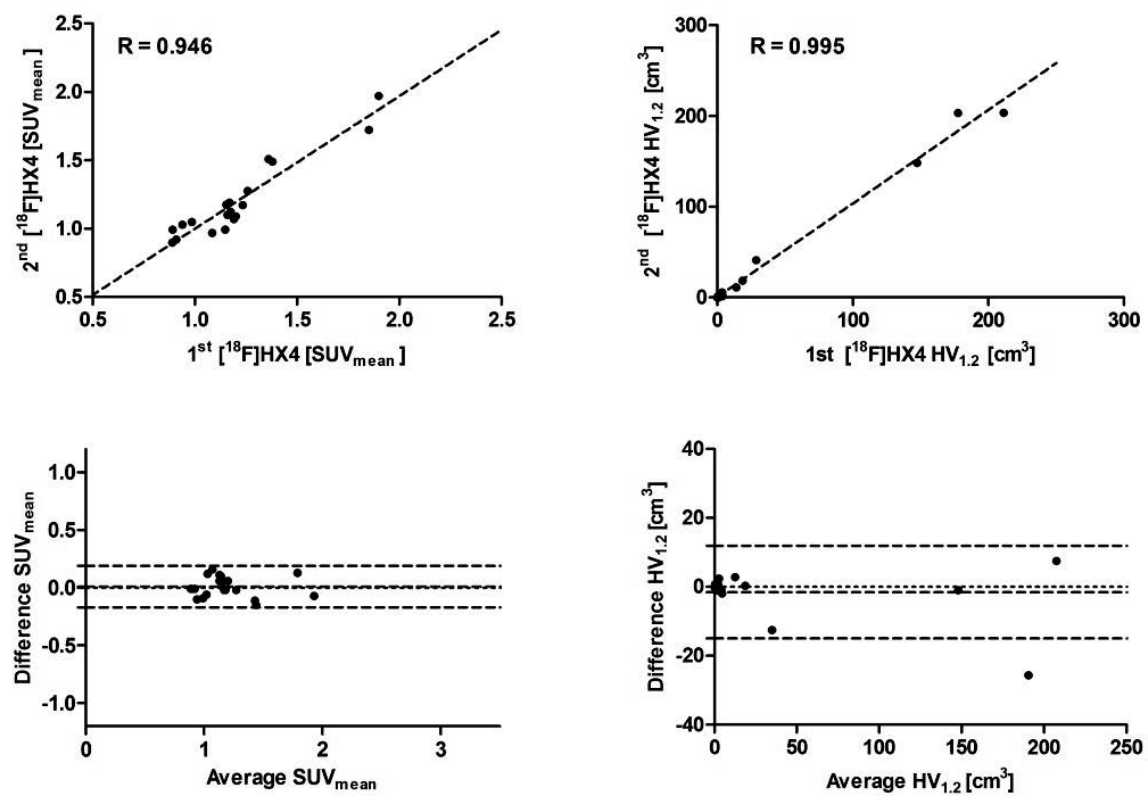

Figure S4.1: Correlation and Bland Altman Plot of image parameters; SUV $V_{\text {mean }}$ and hypoxic tumor volume $\left(H V_{1.2}\right)$ 


\section{REFERENCES}

1. Dubois LJ, Lieuwes NG, Janssen MH, Peeters WJ, Windhorst AD, Walsh JC, et al. Preclinical evaluation and validation of [18F]HX4, a promising hypoxia marker for PET imaging. Proc Natl Acad Sci U S A. 2011;108:14620-5.

2. Chen L, Zhang Z, Kolb HC, Walsh JC, Zhang J, Guan Y. (1)(8)F-HX4 hypoxia imaging with PET/CT in head and neck cancer: a comparison with (1)(8)F-FMISO. Nucl Med Commun. 2012;33:1096-102.

3. Wouters BG, van den Beucken T, Magagnin MG, Lambin P, Koumenis C. Targeting hypoxia tolerance in cancer. Drug resistance updates : reviews and commentaries in antimicrobial and anticancer chemotherapy. 2004;7:25-40.

4. Walsh JC, Lebedev A, Aten E, Madsen K, Marciano L, Kolb HC. The Clinical Importance of Assessing Tumor Hypoxia: Relationship of Tumor Hypoxia to Prognosis and Therapeutic Opportunities. Antioxidants \& redox signaling. 2014.

5. Wouters BG, Koritzinsky M, Chiu RK, Theys J, Buijsen J, Lambin P. Modulation of cell death in the tumor microenvironment. Seminars in radiation oncology. 2003;13:31-41.

6. Nordsmark M, Overgaard J. A confirmatory prognostic study on oxygenation status and loco-regional control in advanced head and neck squamous cell carcinoma treated by radiation therapy. Radiother Oncol. 2000;57:39-43.

7. Thorwarth D, Eschmann SM, Paulsen F, Alber M. Hypoxia dose painting by numbers: a planning study. Int J Radiat Oncol Biol Phys. 2007;68:291-300.

8. Sun JD, Liu Q, Wang J, Ahluwalia D, Ferraro D, Wang Y, et al. Selective tumor hypoxia targeting by hypoxia-activated prodrug $\mathrm{TH}-302$ inhibits tumor growth in preclinical models of cancer. Clin Cancer Res. 2012;18:758-70.

9. Wilson WR, Hay MP. Targeting hypoxia in cancer therapy. Nature reviews Cancer. 2011;11:393-410.

10. Rami M, Dubois L, Parvathaneni NK, Alterio V, van Kuijk SJ, Monti SM, et al. Hypoxiatargeting carbonic anhydrase IX inhibitors by a new series of nitroimidazolesulfonamides/sulfamides/sulfamates. Journal of medicinal chemistry. 2013;56:8512-20.

11. Dubois L, Peeters SG, van Kuijk SJ, Yaromina A, Lieuwes NG, Saraya R, et al. Targeting carbonic anhydrase IX by nitroimidazole based sulfamides enhances the therapeutic effect of tumor irradiation: a new concept of dual targeting drugs. Radiother Oncol. 2013;108:523-8.

12. Thorwarth D, Alber M. Implementation of hypoxia imaging into treatment planning and delivery. Radiother Oncol. 2010;97:172-5.

13. Lambin P, van Stiphout RG, Starmans MH, Rios-Velazquez E, Nalbantov G, Aerts HJ, et al. Predicting outcomes in radiation oncology--multifactorial decision support systems. Nature reviews Clinical oncology. 2013;10:27-40.

14. van Loon J, Janssen $M H$, Ollers $M$, Aerts $H J$, Dubois $L$, Hochstenbag $M$, et al. PET imaging of hypoxia using [18F]HX4: a phase I trial. Eur J Nucl Med Mol Imaging. 2010;37:1663-8.

15. Doss M, Zhang JJ, Belanger MJ, Stubbs JB, Hostetler ED, Alpaugh K, et al. Biodistribution and radiation dosimetry of the hypoxia marker $18 \mathrm{~F}-\mathrm{HX} 4$ in monkeys and humans determined by using whole-body PET/CT. Nuclear medicine communications. 2010;31:1016-24. 
16. Zegers $\mathrm{CM}$, van Elmpt W, Wierts R, Reymen B, Sharifi H, Ollers MC, et al. Hypoxia imaging with [(18)F]HX4 PET in NSCLC patients: Defining optimal imaging parameters. Radiother Oncol. 2013;109:58-64.

17. Zips D, Zophel K, Abolmaali N, Perrin R, Abramyuk A, Haase R, et al. Exploratory prospective trial of hypoxia-specific $\mathrm{PET}$ imaging during radiochemotherapy in patients with locally advanced head-and-neck cancer. Radiother Oncol. 2012;105:21-8.

18. Okamoto S, Shiga T, Yasuda K, Ito YM, Magota K, Kasai K, et al. High reproducibility of tumor hypoxia evaluated by $18 \mathrm{~F}$-fluoromisonidazole PET for head and neck cancer. J Nucl Med. 2013;54:201-7.

19. Nehmeh SA, Lee NY, Schroder H, Squire O, Zanzonico PB, Erdi YE, et al. Reproducibility of intratumor distribution of (18)F-fluoromisonidazole in head and neck cancer. Int J Radiat Oncol Biol Phys. 2008;70:235-42.

20. Busk M, Mortensen LS, Nordsmark M, Overgaard J, Jakobsen S, Hansen KV, et al. PET hypoxia imaging with FAZA: reproducibility at baseline and during fractionated radiotherapy in tumour-bearing mice. Eur J Nucl Med Mol Imaging. 2013;40:186-97.

21. de Langen AJ, Vincent A, Velasquez LM, van Tinteren H, Boellaard R, Shankar LK, et al. Repeatability of 18F-FDG uptake measurements in tumors: a metaanalysis. J Nucl Med. 2012;53:701-8.

22. Peeters SG, Zegers CM, Yaromina A, van Elmpt W, Dubois L, Lambin P. Current preclinical and clinical applications of hypoxia PET imaging using 2-nitroimidazoles. Q J Nucl Med Mol Imaging. 2014.

23. Peeters SG, Zegers CM, Lieuwes NG, van Elmpt W, Eriksson J, van Dongen GA, et al. A Comparative Study of the Hypoxia PET Tracers [F]HX4, [F]FAZA, and [F]FMISO in a Preclinical Tumor Model. Int J Radiat Oncol Biol Phys. 2014.

24. Bittner MI, Wiedenmann N, Bucher S, Hentschel M, Mix M, Weber WA, et al. Exploratory geographical analysis of hypoxic subvolumes using 18F-MISO-PET imaging in patients with head and neck cancer in the course of primary chemoradiotherapy. Radiother Oncol. 2013;108:511-6.

25. Mortensen LS, Johansen J, Kallehauge J, Primdahl H, Busk M, Lassen P, et al. FAZA $\mathrm{PET} / \mathrm{CT}$ hypoxia imaging in patients with squamous cell carcinoma of the head and neck treated with radiotherapy: results from the DAHANCA 24 trial. Radiother Oncol. 2012;105:14-20.

26. Servagi-Vernat S, Differding S, Hanin FX, Labar D, Bol A, Lee JA, et al. A prospective clinical study of F-FAZA PET-CT hypoxia imaging in head and neck squamous cell carcinoma before and during radiation therapy. Eur J Nucl Med Mol Imaging. 2014.

27. Zegers CM, van Elmpt W, Reymen B, Even AJ, Troost EG, Ollers MC, et al. In vivo quantification of hypoxic and metabolic status of NSCLC tumors using [18F]HX4 and [18F]FDG-PET/CT imaging. Clin Cancer Res. 2014;20:6389-97.

28. Petit SF, Dekker AL, Seigneuric R, Murrer L, van Riel NA, Nordsmark M, et al. Intravoxel heterogeneity influences the dose prescription for dose-painting with radiotherapy: a modelling study. Physics in medicine and biology. 2009;54:2179-96.

29. Clausen MM, Hansen AE, Af Rosenschold PM, Kjaer A, Kristensen AT, McEvoy FJ, et al. Dose escalation to high-risk sub-volumes based on non-invasive imaging of hypoxia and glycolytic activity in canine solid tumors: a feasibility study. Radiation oncology. 2013;8:262. 
94 |Chapter 4

30. Zegers CM, van Elmpt W, Wierts R, Reymen B, Sharifi H, Ollers MC, et al. Hypoxia imaging with $[(1)(8) \mathrm{F}] \mathrm{HX} 4$ PET in NSCLC patients: defining optimal imaging parameters. Radiother Oncol. 2013;109:58-64.

31. Boellaard R. Standards for PET image acquisition and quantitative data analysis. J Nucl Med. 2009;50 Suppl 1:11S-20S. 


\section{CHAPTER}

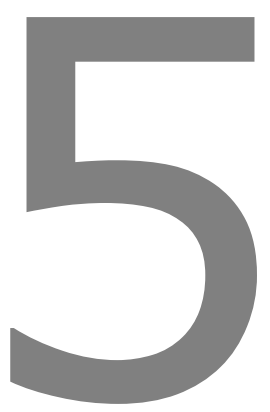

Hypoxia imaging with $\left[{ }^{18} \mathrm{~F}\right] \mathrm{HX} 4 \mathrm{PET}$ in NSCLC: defining optimal imaging parameters

Catharina M.L. Zegers, Wouter van Elmpt, Roel Wierts, Bart Reymen, Hoda Sharifi, Michel C. Öllers, Frank Hoebers, Esther G.C. Troost, Rinus Wanders, Angela van Baardwijk, Jonas Eriksson, Bert Windhorst, Felix M. Mottaghy, Dirk De Ruysscher, Philippe Lambin

Published in: Radiother Oncol. 2013; 109(1):58-64 
96 |Chapter 5

\section{ABSTRACT}

Background and Purpose: $\left[{ }^{18} \mathrm{~F}\right] \mathrm{HX} 4$ is a promising hypoxia PET-tracer. Uptake, spatiotemporal stability and optimal acquisition parameters for $\left[{ }^{18} \mathrm{~F}\right] \mathrm{HX} 4$ PET imaging were evaluated in non-small cell lung cancer (NSCLC) patients.

Materials and methods: $\left[{ }^{18} \mathrm{~F}\right] \mathrm{HX} 4 \mathrm{PET} / \mathrm{CT}$ images of 15 NSCLC patients were acquired $2 \mathrm{~h}$ and $4 \mathrm{~h}$ after injection (p.i.). Maximum standardized-uptake-value $\left(S U V_{\max }\right)$, tumor-toblood-ratio $\left(\mathrm{TBR}_{\max }\right)$, hypoxic fraction (HF) and contrast-to-noise-ratio (CNR) were determined for all lesions. To evaluate spatio-temporal stability, DICE-similarity and Pearson correlation coefficients were calculated. Optimal acquisition-duration was assessed by comparing $30,20,10$ and $5 \mathrm{~min}$ acquisitions.

Results: Considerable uptake (TBR > 1.4) was observed in $18 / 25$ target lesions. TBR $_{\max }$ increased significantly from $2 \mathrm{~h}(1.6 \pm 0.3)$ to $4 \mathrm{~h}$ p.i. $(2.0 \pm 0.6)$. Uptake patterns at $2 \mathrm{~h}$ and $4 \mathrm{~h}$ p.i. showed a strong correlation $(R=0.77 \pm 0.10)$ with a DICE similarity coefficient of $0.69 \pm 0.08$ for the $30 \%$ highest uptake volume. Reducing acquisition-time resulted in significant changes in SUV $V_{\max }$ and CNR. TBR max $_{\text {ax }}$ and HF were only affected for scan-times of $5 \mathrm{~min}$.

Conclusions: The majority of NSCLC lesions showed considerable $\left[{ }^{18} \mathrm{~F}\right] \mathrm{HX} 4$ uptake. The heterogeneous uptake pattern was stable between $2 \mathrm{~h}$ and $4 \mathrm{~h}$ p.i. $\left[{ }^{18} \mathrm{~F}\right] \mathrm{HX} 4$ PET imaging at $4 \mathrm{~h}$ p.i. is superior to $2 \mathrm{~h}$ p.i. to reach highest contrast. Acquisition time may be reduced to 10 min without significant effects on $\mathrm{TBR}_{\max }$ and $\mathrm{HF}$. 


\section{INTRODUCTION}

Radiotherapy combined with chemotherapy is the standard treatment modality for patients with locally advanced non-small cell lung cancer (NSCLC). Unfortunately, after treatment, progression free survival is short with a median of 14 months (1). Tumor cell hypoxia is known to be a major factor that negatively influences treatment effectiveness, it promotes resistance to radiotherapy and chemotherapy and increases tumor aggressiveness, angiogenesis, and metastatic potential, resulting in a poor prognosis. $(2,3)$.

Detection and quantification of tumor cell hypoxia using non-invasive positron emission tomography (PET) could help selecting patients who may benefit from treatment adaptation counteracting hypoxia $(4,5)$. The selective binding and retention of 2-nitroimidazoles allows detection and quantification of tumor hypoxia with PET imaging prior to and during treatment (6-11). In addition, it provides the opportunity to display the spatial distribution of hypoxia, which is essential for its integration in radiation dose distribution (12). An increased radiation dose to the radio-resistant/hypoxic areas may result in an increased local control (13-15). Therefore accurate identification and stable detection of the intratumor hypoxic sub-volumes is of importance (16).

Several 2-nitroimidazoles, labeled with fluor-18 $\left[{ }^{18} \mathrm{~F}\right]$, have already been applied in patients to identify hypoxia (17). The 2-nitroimidazole nucleoside analog: 3-[ $\left.{ }^{18} \mathrm{~F}\right]$ fluoro-2-(4((2-nitro-1H-imidazol-1-yl)methyl)-1H-1,2,3-triazol-1-yl)propan-1-ol [ $\left.{ }^{18} \mathrm{~F}\right] \mathrm{HX} 4$, was developed as a potential marker to visualize hypoxic tumor cells (18). It has a high water solubility and fast clearance from non-hypoxic tissue, therefore generating a tracer with preferred pharmacokinetic properties (18-20). Previous preclinical and clinical studies have shown that $\left[{ }^{18} \mathrm{~F}\right] \mathrm{HX} 4$ is a promising and non-toxic tracer to visualize tumor hypoxia (18-21). In a rat rhabdomyosarcoma model the $\left[{ }^{18} \mathrm{~F}\right] \mathrm{HX} 4$ PET contrast (tumor-to-blood ratio; TBR) increased significantly over time, reaching a plateau and optimal imaging at $4 \mathrm{~h}$ after injection (18). In an inter-patient comparison $\left[{ }^{18} \mathrm{~F}\right] \mathrm{HX} 4$ yielded a similar tumor-to-muscle ratio at $1.5 \mathrm{~h}$ post-injection (p.i.) than $\left[{ }^{18} \mathrm{~F}\right] \mathrm{MISO}$ at $2 \mathrm{~h}$ p.i., suggesting that $\left[{ }^{18} \mathrm{~F}\right] \mathrm{HX} 4$ may be used with a shorter injection-acquisition time than $\left[{ }^{18} \mathrm{~F}\right] \mathrm{MISO}(21)$.

The aims of this study were to evaluate the $\left[{ }^{18} \mathrm{~F}\right] \mathrm{HX} 4$ uptake in NSCLC patients, to report spatio-temporal stability, compare imaging at different time-points and evaluate the possibility to reduce acquisition time. 


\section{MATERIALS AND METHODS}

\section{Patients}

Fifteen NSCLC patients analyzed in this study were included in the PET-Boost (15) (NCT01024829) or Nitroglycerin trial (NCT01210378), approved by the appropriate Medical Ethics Review Committee. Written informed consent was obtained from all patients before study entry. $\left[{ }^{18} \mathrm{~F}\right] \mathrm{HX} 4 \mathrm{PET} / \mathrm{CT}$ imaging was acquired at baseline, i.e. before the start of external beam radiotherapy. However, patients were treated with concurrent or sequential chemoradiation and received at least one course of chemotherapy before the start of radiotherapy and $\left[{ }^{18} \mathrm{~F}\right] \mathrm{HX} 4 \mathrm{PET} / \mathrm{CT}$ imaging.

Table 5.1: Patient characteristics

\begin{tabular}{|c|c|c|c|c|c|c|c|c|}
\hline Patient & Age & Gender & TNM & Stage & Pathology & GTV & size GTV $\left[\mathrm{cm}^{3}\right]$ & $\begin{array}{l}\text { Prior treatment } \\
\text { [no. chemo] }\end{array}$ \\
\hline \multirow[t]{2}{*}{1} & 55 & $M$ & T2aN3M0 & IIIB & LCC & prim & 48 & 1 \\
\hline & & & & & & In & 190 & \\
\hline \multirow[t]{2}{*}{2} & 59 & $\mathrm{~F}$ & T4N1M0 & IIIB & $A C$ & prim & 328 & 3 \\
\hline & & & & & & In & 25 & \\
\hline \multirow[t]{2}{*}{3} & 72 & $M$ & T2N3M0 & IIIB & $A C$ & prim & 42 & 1 \\
\hline & & & & & & $\ln$ & 51 & \\
\hline \multirow[t]{2}{*}{4} & 60 & $M$ & T4N1M0 & IIIB & $A C$ & prim & 859 & 1 \\
\hline & & & & & & $\ln *$ & 8 & \\
\hline \multirow[t]{2}{*}{5} & 65 & $M$ & T3N2MO & IIIA & SCC & prim & 148 & 1 \\
\hline & & & & & & $\ln$ & 39 & \\
\hline \multirow[t]{2}{*}{6} & 66 & $M$ & T2N3M0 & IIIB & - & prim & 78 & 1 \\
\hline & & & & & & $\ln$ & 13 & \\
\hline \multirow[t]{2}{*}{7} & 51 & $\mathrm{~F}$ & T3N2M0 & IIIA & LCC & prim & 150 & 2 \\
\hline & & & & & & $\ln$ & 11 & \\
\hline 8 & 82 & M & T3NOMO & IIB & $A C$ & prim & 63 & 3 \\
\hline \multirow[t]{2}{*}{9} & 66 & $M$ & T4N3M0 & IIIB & $A C$ & prim & 65 & 2 \\
\hline & & & & & & $\ln *$ & 3 & \\
\hline \multirow[t]{2}{*}{10} & 62 & $\mathrm{~F}$ & T4N2M0 & IIIB & SCC & prim & 150 & 1 \\
\hline & & & & & & $\ln *$ & 5 & \\
\hline \multirow[t]{2}{*}{11} & 77 & M & T3N2M0 & IIIA & LCC & prim & 299 & 1 \\
\hline & & & & & & $\ln *$ & 7 & \\
\hline \multirow[t]{2}{*}{12} & 64 & $M$ & T4N2M0 & IIIB & SCC & prim & 212 & 1 \\
\hline & & & & & & $\ln$ & 13 & \\
\hline \multirow[t]{2}{*}{13} & 60 & $M$ & T4N2M0 & IIIB & $A C$ & prim & 53 & 2 \\
\hline & & & & & & In & 17 & \\
\hline \multirow[t]{2}{*}{14} & 47 & $F$ & T4N3M0 & IIIB & $A C$ & prim & 125 & 1 \\
\hline & & & & & & In & 204 & \\
\hline \multirow[t]{2}{*}{15} & 40 & $M$ & T4N2M1 & IV & $A C$ & prim & 78 & 3 \\
\hline & & & & & & In & 35 & \\
\hline
\end{tabular}

$\mathrm{LCC}=$ large cell carcinoma, $\mathrm{AC}=$ adenocarcinoma, $\mathrm{SCC}=$ squamous cell carcinoma prim = primary lesion, $\mathrm{In}=$ involved lymph nodes, ${ }^{*}$ lesions with a volume $<10 \mathrm{~cm}^{3}$ 


\section{PET/CT imaging}

Images were acquired on a Philips Gemini TF 64 PET/CT scanner (Philips Healthcare, Best, The Netherlands) with an axial field of view of $18 \mathrm{~cm}$, slice thickness of $4 \mathrm{~mm}$, in plane pixel spacing of $4 \mathrm{~mm}$ and a spatial resolution of approximately $5 \mathrm{~mm}$ FWHM. CT based attenuation correction and scatter correction (SS-SIMUL) were performed. The PET images were reconstructed using 3D ordered-subset iterative time-of-flight reconstruction technique (BLOB-OS-TF) using 3 iterations and 33 subsets. The patient was scanned in radiotherapy position, positioned on a flat tabletop using a movable laser alignment system with the arms in an arm-support positioned above the head. The field of view for CT and PET imaging was positioned on the primary tumor. $\left[{ }^{18} \mathrm{~F}\right] \mathrm{HX} 4$ was produced as described in previous publications (18-21). The injected activity of $\left[{ }^{18} \mathrm{~F}\right] \mathrm{HX} 4$ was $423 \pm 72 \mathrm{MBq}$ based on a previous phase I trial(19). After intravenous administration of $\left[{ }^{18} \mathrm{~F}\right] \mathrm{HX} 4, \mathrm{PET} / \mathrm{CT}$ imaging was performed at $2 \mathrm{~h}$ and $4 \mathrm{~h}$ p.i. A single bed-position PET was acquired with a total acquisition time of $30 \mathrm{~min}$.

\section{Analysis}

Gross tumor volumes of the primary tumor $\left(G T V_{\text {prim }}\right)$ and involved lymph nodes $\left(G T V_{\text {In }}\right)$ were defined by an experienced radiation oncologist and evaluated by a second radiation oncologist, on the $\left[{ }^{18} \mathrm{~F}\right]$ fluorodeoxyglucose (FDG) PET/CT scan used for radiotherapy planning purposes, acquired in the same week as the $\left[{ }^{18} \mathrm{~F}\right] \mathrm{HX} 4 \mathrm{PET} / \mathrm{CT}$. [ $\left.{ }^{18} \mathrm{~F}\right] \mathrm{FDG}$-based GTVs were copied to the $\left[{ }^{18} \mathrm{~F}\right] \mathrm{HX} 4 \mathrm{PET} / \mathrm{CT}$ images by rigid registration and a visual check was performed. No matching problems occurred. In addition a volume of interest (VOI) in the aorta was defined as background region.

\section{Scan time point}

The optimal imaging time point was evaluated by determining image parameters in the 2 $\mathrm{h}$ and $4 \mathrm{~h}$ p.i. $\left[{ }^{18} \mathrm{~F}\right] \mathrm{HX} 4$ PET scans. The mean and maximum standardized uptake values (SUV $_{\text {mean }}$ and SUV $V_{\text {max }}$ respectively) in the GTVs and aorta were extracted (PMOD v3.0, Zurich, Switzerland). The tumor-to-background ratio $\left(T B R_{\max }\right)$ was defined as the ratio of

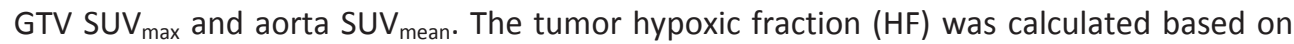
the $\left[{ }^{18} \mathrm{~F}\right] \mathrm{HX} 4$ uptake. A threshold for the definition of hypoxia has not been formally established, based on previous publications, a TBR larger than 1.4 was used as the standard threshold to define tumor hypoxia $(18,22-24)$. In addition, the HF was calculated based on a threshold ranging from TBR $>1.1$ to TBR $>1.6$.

\section{Spatio-temporal stability}

The spatio-temporal stability was evaluated using two methods, first by calculating the DICE similarity coefficient of the hypoxic volumes and second with a voxel-wise comparison of the $\left[{ }^{18} \mathrm{~F}\right] \mathrm{HX} 4$ uptake at $2 \mathrm{~h}$ and $4 \mathrm{~h}$ p.i. For both methods the $\mathrm{CT}$ acquired at $2 \mathrm{~h} \mathrm{p.i.}$ $\left(\mathrm{CT}_{2 \mathrm{~h}}\right)$ was rigidly registered to the $\mathrm{CT}$ at $4 \mathrm{~h}$ p.i. $\left(\mathrm{CT}_{4 \mathrm{~h}}\right)$. The resulting deformation field was applied to co-register the PET at $2 \mathrm{~h}$ p.i. $\left(\mathrm{PET}_{2 \mathrm{~h}}\right)$ to the PET at $4 \mathrm{~h}$ p.i. $\left(\mathrm{PET}_{4 h}\right)$.

To calculate the DICE similarity coefficient; First the percentile of the GTV with the highest uptake on $\mathrm{PET}_{2 \mathrm{~h}}$ and $\mathrm{PET}_{4 \mathrm{~h}}$ was defined, by using percentiles ranging from $50 \%$ to $90 \%$. Second, only for lesions with a HF $>5 \%$, the hypoxic volume on the $\mathrm{PET}_{4 \mathrm{~h}}$ and its corre- 
sponding high uptake volume on the $\mathrm{PET}_{2 \mathrm{~h}}$ were defined. The resulting high uptake volumes (UV) are represented by $U_{2}$ and $U_{2} V_{4 h}$. DICE was calculated using:

$$
D I C E=2 \frac{\mathrm{UV}_{2 \mathrm{~h}} \cap \mathrm{UV}_{4 \mathrm{~h}}}{\mathrm{UV}_{2 \mathrm{~h}}+\mathrm{UV}_{4 \mathrm{~h}}}
$$

Furthermore, a voxel-wise comparison of the $\left[{ }^{18} \mathrm{~F}\right] \mathrm{HX} 4$ PET uptake in the GTV in PET $2 \mathrm{~h}$ and $\mathrm{PET}_{4 h}$ was performed.

\section{Reduced acquisition time}

Because hypoxia PET tracers generally have a low uptake in tumors, prolonged PET acquisition times may be preferred. Additional reconstructions were made with reduced acquisition duration. The full acquisition time of $30 \mathrm{~min}$ was compared to the first 20,10 and 5 min acquisitions by evaluating changes in $\mathrm{SUV}_{\max }, \mathrm{TBR}_{\max }, \mathrm{HF}$ and the contrast-to-noise ratio (CNR) defined as:

$$
C N R=\frac{S U V_{\text {max }} \text { tumor }-S U V_{\text {mean }} \text { aorta }}{S U V_{S D} \text { aorta }}
$$

\section{$\underline{\text { Statistics }}$}

For all parameters mean \pm 1 standard deviation (SD) are reported. A Wilcoxon signed rank test was used to determine significant differences in uptake $\left(S U V_{\max }, T B R_{\max }\right)$ between $2 \mathrm{~h}$ and $4 \mathrm{~h}$ p.i. and to evaluate the effect on image parameters (SUV $V_{\max }, T_{B R} R_{\max }, H F, C N R$ ) when limiting the acquisition time. Linear regression was performed to correlate $\mathrm{SUV}_{\max }$ and $\mathrm{TBR}_{\max }$ from $2 \mathrm{~h}$ to $4 \mathrm{~h}$ p.i. and to quantify the voxel-wise comparison of the $\left[{ }^{18} \mathrm{~F}\right] \mathrm{HX} 4$ uptake within the GTV. Slope and Pearson correlation coefficients were calculated. A $p$ value smaller than 0.05 was assumed to be statistically significant.

\section{RESULTS}

$\left[{ }^{18} \mathrm{~F}\right] \mathrm{HX} 4 \mathrm{PET} / \mathrm{CT}$ imaging of 15 NSCLC patients (11 male, 4 female) was included in the analysis. The mean age of the patients was $62 \pm 10$ years (range 40-82 years). Tumor stage ranged from IIB to IV; pathology being adenocarcinoma $(n=8)$, squamous cell carcinoma $(n=3)$, large cell carcinoma $(n=3)$ or not specified $(n=1)$. All patients were treated with curative intent and had one to three cycles of chemotherapy before hypoxia PET imaging and the start of radiotherapy. Patient characteristics are visualized in Table 5.1. All but one patient had involvement of the lymph nodes $\left(G T V_{I n}\right)$ which were separately analyzed from the primary tumor $\left(G V_{\text {prim }}\right)$. Four involved lymph nodes with a volume $<10 \mathrm{~cm}^{3}$ were excluded, due to potential partial volume effects. As a result 25 target lesions $\left(\mathrm{GTV}_{\text {prim }} n=\right.$ $15, \mathrm{GTV}_{\mathrm{In}} n=10$ ) were selected for analysis, with an average lesion size of $180 \pm 208 \mathrm{~cm}^{3}$ (range $42-859 \mathrm{~cm}^{3}$ ) for primary tumor and $60 \pm 74 \mathrm{~cm}^{3}$ (range $11-204 \mathrm{~cm}^{3}$ ) for the sum of the pathological lymph nodes.

For two patients (P3 and P4) the SUV values could not be determined due to an undefined amount of residual activity in the administration system. For these patients, absolute SUV 
was calculated based on the estimated activity in the syringe before injection, but excluded from statistical analysis. TBR $\max _{\max } \mathrm{HF}$ and CNR calculations were not affected by this spill and therefore included in the analysis.

Tumor hypoxia, defined as $\mathrm{TBR}_{\max }>1.4$ on the $4 \mathrm{~h}$ p.i. acquisition, was observed in $80 \%$ $(12 / 15)$ of the primary tumors and $60 \%(6 / 10)$ of lymph node regions. An example of a $2 \mathrm{~h}$ and $4 \mathrm{~h}$ p.i. PET/CT image is shown in Figure 5.1. Comparing $2 \mathrm{~h}$ with $4 \mathrm{~h} \mathrm{p}$.i., there was a high correlation for both $\operatorname{SUV}_{\max }(R=0.96)$ and $\operatorname{TBR}_{\max }(R=0.94)$.

Within the hypoxic lesions, the tumor $\mathrm{SUV}_{\max }$ decreased between $2 \mathrm{~h}$ p.i. and $4 \mathrm{~h}$ p.i. for both $\operatorname{GTV}_{\text {prim }}(1.5 \pm 0.4$ to $1.4 \pm 0.4 ; P<0.001)$ and $\operatorname{GTV}_{\text {In }}(1.4 \pm 0.2$ to $1.2 \pm 0.2 ; P=0.16)$. However, due to clearance of $\left[{ }^{18} \mathrm{~F}\right] \mathrm{HX} 4$ in the blood, the $\mathrm{TBR}_{\max }$ increased significantly from $2 \mathrm{~h}$ to $4 \mathrm{~h}$ p.i. $\left(\mathrm{GTV}_{\text {prim: }} 1.6 \pm 0.3\right.$ to $2.0 \pm 0.6 ; P<0.001$ and $\mathrm{GTV}_{\mathrm{In}}$ : $1.5 \pm 0.3$ to $2.1 \pm$ $0.6 ; P=0.03$ ) as shown in Table 5.2 and Figure 5.2. The $S_{U} V_{\text {mean }}$ measured within the aorta VOI was $1.0 \pm 0.2$ and $0.7 \pm 0.2$ at $2 \mathrm{~h}$ p.i. and $4 \mathrm{~h}$ p.i respectively. Assuming an exponential clearance of HX4 in the blood, this resulted in an estimated biological half life of $4.3 \mathrm{~h}$.

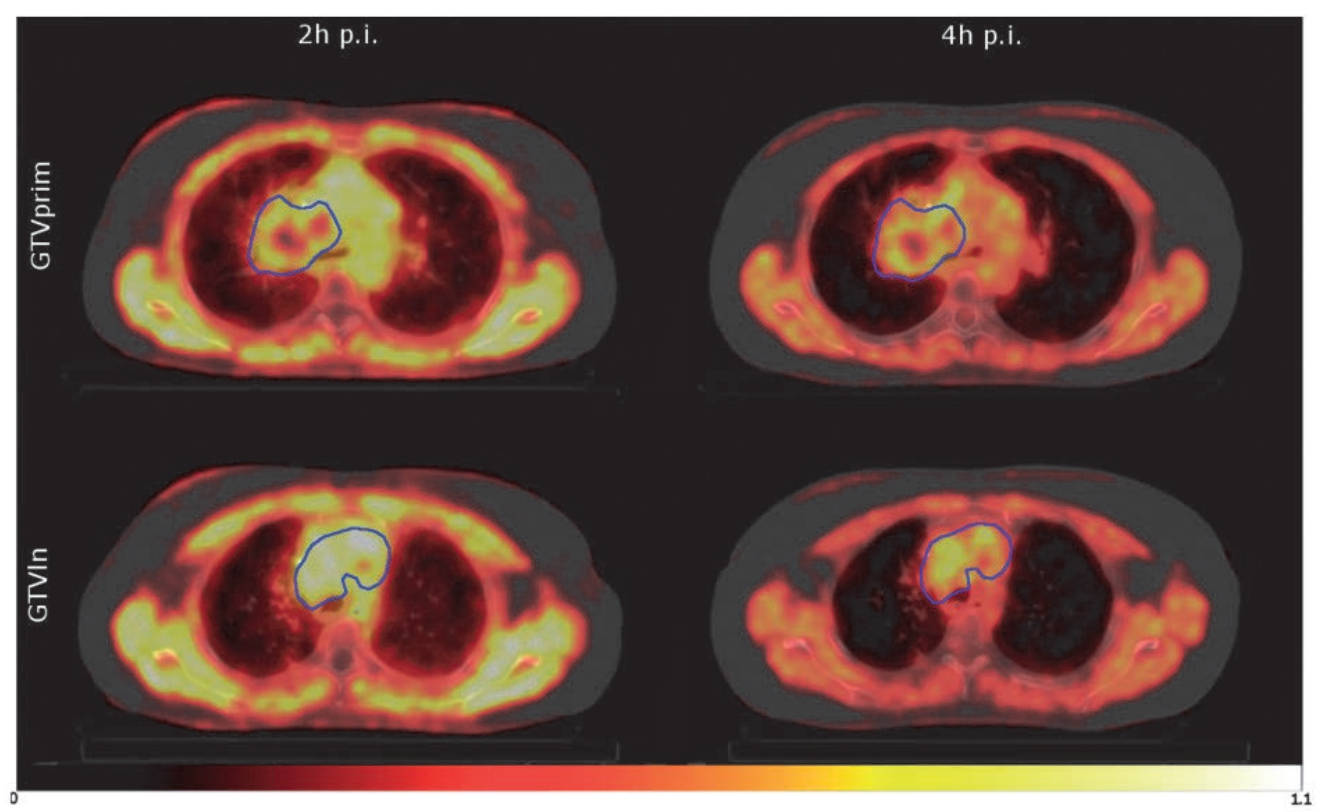

Figure 5.1: Example of two transversal slices of the [18F]HX4 CT/PET image of patient 14 at $2 h$ and $4 h$ p.i. showing the primary tumor (GTV prim) and involved lymph nodes $\left(G T V_{l n}\right)$ 
102 Chapter 5

Table 5.2: Target lesion characteristics at $2 h$ and $4 h$ p.i.

\begin{tabular}{|c|c|c|c|c|c|c|c|c|}
\hline \multirow[b]{2}{*}{ Patient } & \multirow[b]{2}{*}{ Lesion } & \multirow[b]{2}{*}{$\operatorname{GTV}\left[\mathrm{cm}^{3}\right]$} & \multicolumn{2}{|c|}{ Tumour SUV $_{\max }$} & \multicolumn{2}{|c|}{ Aorta $\mathrm{SUV}_{\text {mean }}$} & \multicolumn{2}{|l|}{ TBR } \\
\hline & & & $2 \mathrm{~h}$ p.i. & 4h p.i. & 2h p.i. & 4h p.i. & $2 \mathrm{~h}$ p.i. & 4h p.i. \\
\hline \multirow[t]{2}{*}{1} & prim & 48 & 0.93 & 0.64 & 0.88 & 0.58 & 1.06 & 1.10 \\
\hline & $\ln ^{+}$ & 190 & 1.63 & 1.65 & & & 1.87 & 2.84 \\
\hline \multirow[t]{2}{*}{2} & $\operatorname{prim}^{+}$ & 328 & 2.19 & 1.84 & 0.91 & 0.53 & 2.40 & 3.47 \\
\hline & $\ln ^{+}$ & 25 & 1.63 & 1.36 & & & 1.79 & 2.56 \\
\hline \multirow[t]{2}{*}{3} & $\operatorname{prim}^{+}$ & 42 & $0.73^{x}$ & $0.65^{*}$ & $0.48^{x}$ & $0.36^{*}$ & 1.51 & 1.78 \\
\hline & $\operatorname{Ln}$ & 51 & $0.63^{x}$ & $0.48^{x}$ & & & 1.30 & 1.33 \\
\hline \multirow[t]{2}{*}{4} & $\operatorname{prim}^{+}$ & 859 & $0.87^{\times}$ & $0.85^{\times}$ & $0.51^{*}$ & $0.34^{x}$ & 1.71 & 2.52 \\
\hline & $\ln *$ & 8 & $0.55^{\times}$ & $0.44^{x}$ & & & 1.08 & 1.32 \\
\hline \multirow[t]{2}{*}{5} & $\operatorname{prim}^{+}$ & 148 & 1.47 & 1.30 & 0.98 & 0.71 & 1.50 & 1.83 \\
\hline & $\ln ^{+}$ & 39 & 1.23 & 1.03 & & & 1.26 & 1.45 \\
\hline \multirow[t]{2}{*}{6} & $\operatorname{prim}^{+}$ & 78 & 1.49 & 1.44 & 0.79 & 0.62 & 1.90 & 2.33 \\
\hline & $\operatorname{Ln}$ & 13 & 1.14 & 0.98 & & & 1.45 & 1.58 \\
\hline \multirow[t]{2}{*}{7} & $\operatorname{prim}^{+}$ & 150 & 0.91 & 0.66 & 0.67 & 0.44 & 1.36 & 1.49 \\
\hline & $\operatorname{Ln}$ & 11 & 0.73 & 0.51 & & & 1.08 & 1.15 \\
\hline \multirow[t]{2}{*}{8} & $\operatorname{prim}^{+}$ & 63 & 1.45 & 1.63 & 1.02 & 0.85 & 1.43 & 1.92 \\
\hline & - & & & & & & & \\
\hline \multirow[t]{2}{*}{9} & prim & 65 & 1.01 & 0.76 & 0.85 & 0.60 & 1.19 & 1.26 \\
\hline & $\ln *$ & 3 & 0.81 & 0.59 & & & 0.95 & 0.99 \\
\hline \multirow[t]{2}{*}{10} & $\operatorname{prim}^{+}$ & 150 & 1.28 & 1.09 & 0.96 & 0.62 & 1.34 & 1.75 \\
\hline & $\ln ^{*^{+}}$ & 5 & 1.29 & 1.24 & & & 1.35 & 2.00 \\
\hline \multirow[t]{2}{*}{11} & $\operatorname{prim}^{+}$ & 299 & 1.98 & 1.87 & 1.18 & 1.02 & 1.69 & 1.83 \\
\hline & $\ln *$ & 7 & 1.33 & 1.28 & & & 1.13 & 1.25 \\
\hline \multirow[t]{2}{*}{12} & $\operatorname{prim}^{+}$ & 212 & 2.03 & 1.97 & 1.33 & 1.06 & 1.53 & 1.85 \\
\hline & $\operatorname{Ln}$ & 13 & 1.39 & 1.14 & & & 1.04 & 1.07 \\
\hline \multirow[t]{2}{*}{13} & prim & 53 & 1.00 & 0.76 & 0.86 & 0.56 & 1.17 & 1.34 \\
\hline & $\operatorname{Ln}$ & 17 & 0.89 & 0.70 & & & 1.04 & 1.24 \\
\hline \multirow[t]{2}{*}{14} & $\operatorname{prim}^{+}$ & 125 & 1.12 & 1.02 & 0.81 & 0.54 & 1.37 & 1.90 \\
\hline & $\ln ^{+}$ & 204 & 1.19 & 1.28 & & & 1.46 & 2.37 \\
\hline \multirow[t]{2}{*}{15} & $\operatorname{prim}^{+}$ & 78 & 1.40 & 1.13 & 1.10 & 0.78 & 1.27 & 1.45 \\
\hline & $\ln ^{+}$ & 35 & 1.32 & 1.19 & & & 1.20 & 1.52 \\
\hline \multirow{2}{*}{\multicolumn{2}{|c|}{ Average $\left[>10 \mathrm{~cm}^{2}\right]$}} & $132 \pm$ & $1.34 \pm$ & $1.18 \pm$ & $0.95 \pm$ & $0.69 \pm$ & $1.44 \pm$ & $1.80 \pm$ \\
\hline & & 175 & 0.39 & 0.43 & 0.18 & 0.19 & 0.32 & 0.60 \\
\hline \multirow{2}{*}{\multicolumn{2}{|c|}{$\begin{array}{l}\text { Average [hypoxic \& } \\
>10 \mathrm{~cm}^{2} \text { ] }\end{array}$}} & $169 \pm$ & $1.47 \pm$ & $1.34 \pm$ & & & $1.56 \pm$ & $2.03 \pm$ \\
\hline & & 195 & 0.36 & 0.37 & & & 0.30 & 0.55 \\
\hline
\end{tabular}



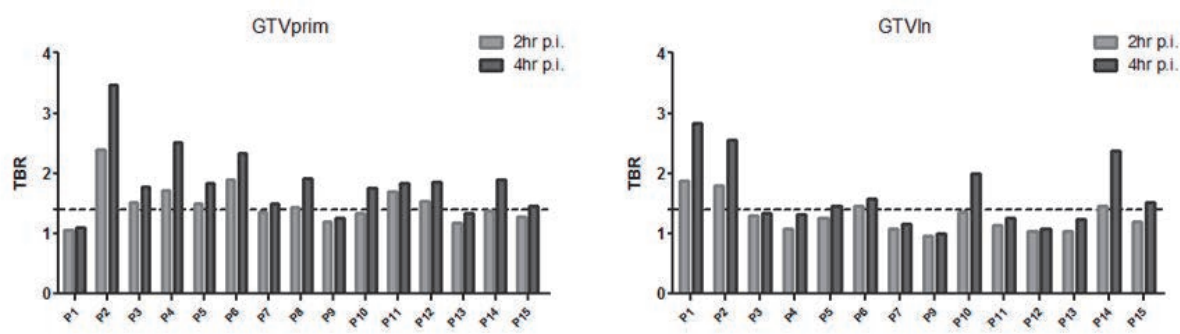

Figure 5.2: $T B R_{\max }$ of the primary tumor $\left(G T V_{\text {prim }}\right)$ and involved lymph nodes (GTV $\left.V_{\text {In }}\right)$ of all patients.

The average HF (TBR >1.4), based on the $4 \mathrm{~h}$ p.i. image, was $15 \pm 19 \%$ (GTV prim, $_{\text {, range }} 0.1$ $66 \%$ ) and $12 \pm 13 \%$ (GTV $V_{I n}$, range $\left.0.3-36 \%\right)$ for the hypoxic lesions. No correlation between the GTV and HF was observed $(R=0.12, P=0.57)$. When applying the same threshold (TBR $>1.4)$ in the $2 \mathrm{~h}$ p.i. images, a lower amount of hypoxic lesions were detected $\left(8 / 15 \mathrm{GTV}_{\text {prim }}\right.$ and $\left.4 / 10 G_{\text {In }}\right)$ and the average $H F$ was reduced to $5 \pm 10 \%\left(G_{\text {TV }}\right.$ prim $)$ and $3 \pm 6 \%\left(G_{\text {TV }}\right)$. Using a threshold of TBR $>1.2$ resulted in a similar hypoxic lesion detection rate (12/15 and $7 / 10$, respectively) and HF (GTV prim $17 \pm 17 \%$ and GTV $_{\text {In }} 10 \pm 10 \%$ ) compared to $4 \mathrm{~h}$ p.i., however the use of this lower threshold resulted in one false positive case. The hypoxic fractions based on thresholds ranging from TBR $>1.1$ to TBR $>1.6$ on both $2 \mathrm{~h}$ and $4 \mathrm{~h}$ p.i. acquisitions are summarized in Table S5.1.

Although the hypoxic lesions showed a heterogeneous $\left[{ }^{18} \mathrm{~F}\right] \mathrm{HX} 4$ uptake pattern, the voxelwise comparison of the $\mathrm{PET}_{2 \mathrm{~h}}$ and $\mathrm{PET}_{4 \mathrm{~h}}$ showed a strong correlation $(R=0.77 \pm 0.10$, range: $0.58-0.94$, slope: $0.72 \pm 0.15$ ), see Figure 5.3 and Table S5.2.. Comparing high uptake percentiles resulted in an average DICE similarity coefficient of $0.79 \pm 0.06,0.75 \pm$ $0.06,0.70 \pm 0.08,0.61 \pm 0.10$ and $0.48 \pm 0.10$ for the highest volume percentiles $50 \%, 60 \%$, $70 \%, 80 \%$ and $90 \%$, respectively. Comparing the hypoxic volumes defined on the $\mathrm{PET}_{4 \mathrm{~h}}$ with the corresponding high uptake volume on the $\mathrm{PET}_{2 \mathrm{~h}}$ provides a DICE similarity coefficient of $0.61 \pm 0.19$ (Table S5.2).
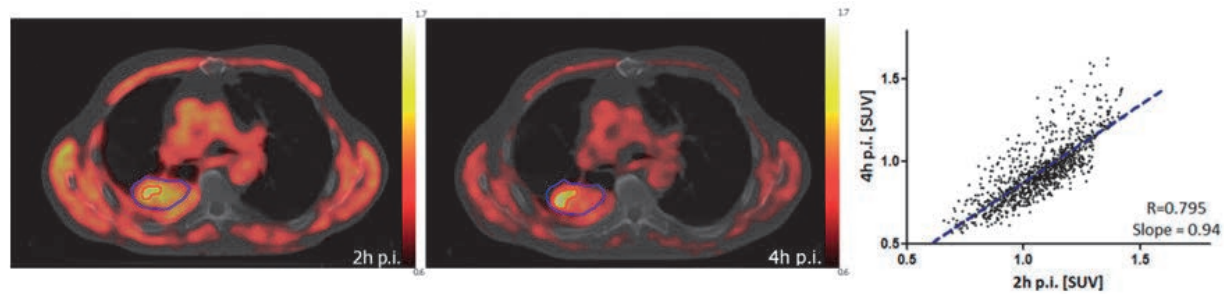

Figure 5.3: Example of patient 8. Left figure shows the $2 \mathrm{~h}$ p.i. [18F]HX4 PET/CT that is rigidly registered to the $4 \mathrm{~h}$ p.i. scan (middle). Visualized are the gross tumor volume (blue) and the hypoxic volume in the $4 h$ p.i. scan and its corresponding high uptake volume in the $2 \mathrm{~h}$ p.i. scan (red). The right figure shows the voxel-wise comparison between $2 h$ and $4 h$ p.i. within the gross tumor volume. 
One patient was excluded from this analysis due to an incomplete 30 min acquisition, resulting in 16 evaluable hypoxic lesions $\left(>10 \mathrm{~cm}^{3}\right)$. Reducing acquisition time from $30 \mathrm{~min}$ to the first 20, 10 and 5 min of the acquisition resulted in an average increase in SUV $\mathrm{max}_{\max }$ of $4 \pm 6 \%(P=0.014), 12 \pm 15 \%(P<0.001)$ and $18 \pm 16 \%(P=0.025)$. TBR $\max$ increased with $2 \pm$ $5 \%(P=0.171), 7 \pm 12 \%(P=0.074)$ and $16 \pm 13 \%(P<0.001)$ for, respectively, the 20, 10 and 5 min acquisition, hence only a significant difference was reached for the 5 min acquisition. The average HF was not significantly different for the $30 \mathrm{~min}$ (11 $\pm 16 \%), 20$ $\min (11 \pm 16 \%)$ and $10 \mathrm{~min}(11 \pm 15 \%)$ acquisition, again for the $5 \mathrm{~min}$ acquisition (average HF: $13 \pm 15 \%)$ the change in HF was significant $(P=0.02)$. The CNR decreased from $9.5 \pm$ 4.1 (30 min) to $8.7 \pm 4.2$ (20 $\mathrm{min} ; P=0.02), 7.8 \pm 4.7$ (10 $\mathrm{min} ; P<0.01)$ and $6.6 \pm 3.2$ (5 min; $P<0.001)$. Images of an example patient are visualized in Figure 5.4 .

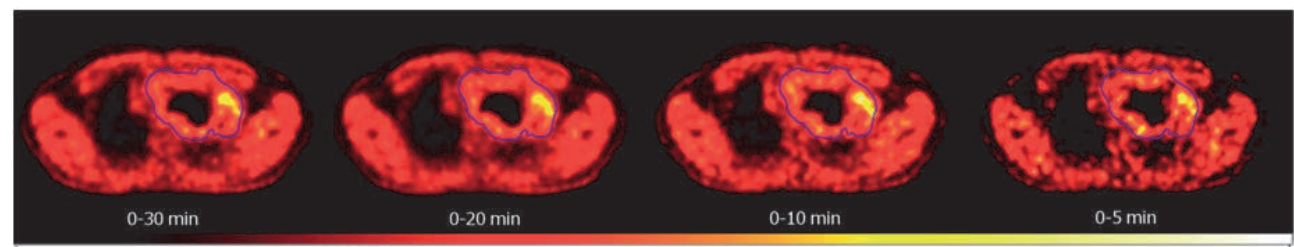

Figure 5.4: Transversal [18F]HX4 PET slice of patient 4 at 4 h p.i., reconstructed with the total acquisition of 30 min or the first 20, 10 and $5 \mathrm{~min}$.

\section{DISCUSSION}

The aims of this study were to evaluate $\left[{ }^{18} \mathrm{~F}\right] \mathrm{HX} 4$ uptake in NSCLC, report spatio-temporal stability, compare imaging at different time-points and evaluate the possibility to reduce acquisition time. Based on this, we want to generate recommendations for future PET imaging with $\left[{ }^{18} \mathrm{~F}\right] \mathrm{HX} 4$. $\left[{ }^{18} \mathrm{~F}\right] \mathrm{HX} 4$ was developed to provide a hypoxia PET tracer with preferred pharmacokinetic and clearance properties compared to other available nitroimidazoles. Based on the current population the biological half-life of HX4 in the blood is approximately $4.3 \mathrm{~h}$. Which is a 3 -fold faster clearance in comparison to [ $\left.{ }^{18} \mathrm{~F}\right] \mathrm{MISO}$, reported to have a biological half life of $12-13 \mathrm{~h}(25,26)$. In a clinical trial, it was shown that $\left[{ }^{18} \mathrm{~F}\right] \mathrm{HX} 4$ provides the same image contrast as $\left[{ }^{18} \mathrm{~F}\right] \mathrm{MISO}$ at an earlier time-point after injection (21), a characteristic beneficial for practical reasons. However, previous preclinical and clinical studies have also shown that for both FMISO $(6,27)$ and $\operatorname{HX} 4(18,19)$ the later scan time-points are optimal in order to reach a higher image contrast. In the current study the image contrast $\left(\mathrm{TBR}_{\max }\right)$ increased from $2 \mathrm{~h}$ to $4 \mathrm{~h}$ p.i. confirming the pre-clinical results, i.e. $\left[{ }^{18} \mathrm{~F}\right] \mathrm{HX} 4$ scanning at $4 \mathrm{~h}$ p.i. provides a better opportunity to identify hypoxic areas. In preclinical setting a TBR plateau was reached at $4 \mathrm{~h}$ p.i., however it is still unknown if in the clinical situation, hypoxia imaging at a timepoint later than $4 \mathrm{~h}$ p.i. could provide an enhanced image contrast(18).

In literature no evaluation has been performed comparing all hypoxia PET tracers in the same tumor model or patient population (17). Hence, it is difficult to compare the current results to clinical trials with other hypoxia tracers used in NSCLC imaging. Other studies 
allowed different treatment modalities before imaging, with differences in scan time p.i. or definition of background tissue (muscle $(7)$, mediastinum $(6,28)$, venous blood sampling $(22,29)$, lung $(30)$, heart (31), not specified (32)). Nevertheless it seems that the average SUV $_{\max }$ resulting from the current analysis are similar compared to other trials with $\left[{ }^{18}\right.$ F]FMISO $(6-8,30)$, FAZA (32) or FETNIM (29).

The definition of the hypoxic volume in the literature is diverse and based on different tracers. For $\left[{ }^{18} \mathrm{~F}\right]$ FMISO imaging, a comparison with immunohistochemistry (24), normal tissue differences (22) and in vivo bio-distribution data (23) a threshold of TBR $>1.4$ can be supported. For $\left[{ }^{18} \mathrm{~F}\right] \mathrm{HX} 4$ imaging a good correlation with immunohistochemistry was observed when $\mathrm{HX} 4$ positive voxels (TBR $>1.4$ ) were compared to pimonidazole staining (18), however the optimal TBR in a clinical setting is still under investigation. Using the TBR $>1.4$ threshold a significant amount of tumor hypoxia was observed in $80 \%$ of the primary tumors and $60 \%$ of the involved lymph nodes. Note that although the lesion size of GTV was in general smaller than GTV $_{\text {prim, }}$ still no relationship was found between GTV and HF. An average HF of $14 \pm 17 \%$ was observed, which is lower in comparison to a previous study of Rasey et al. (22) where a median HF of $58 \%$ was reported. The discrepancy might be explained due to the fact that all patients in the current study had at least one cycle of chemotherapy before $\left[{ }^{18} \mathrm{~F}\right] \mathrm{HX} 4$ PET scans, which may reduce tumor hypoxia $(9,33)$, resulting in a lower SUV $V_{\max }$, TBR and HF. Note that, this is a situation frequently occurring in clinical practice. However, the hypoxic status of the lesion was assessed before the start of radiotherapy, which is of utmost importance in dose redistribution strategies.

The TBR threshold of 1.4 should not be interpreted as a rigid value to determine tumor hypoxia. Due to tracer kinetics, the threshold should be optimized based on scan time post-injection. An alternative threshold of TBR $>1.2$, also used in several trials $(31,34,35)$, provides in the current study a similar amount of detected lesions at $2 \mathrm{~h}$ p.i. in comparison to $4 \mathrm{~h}$ p.i. using a TBR $>1.4$, however the risk of misclassifying lesions increases. This might also explain the difference between the studies of Nehmeh et al. (34) and Okamoto et al. (36) using $\left[{ }^{18} \mathrm{~F}\right] \mathrm{MISO}$ PET imaging in head and neck cancer patients. Nehmeh et al. used a threshold of TBR $>1.2$ and observed that the measured fractional hypoxic volumes were variable over time. Whereas Okamoto et al. using a threshold of TBR $>1.4$ found a high reproducibility of tumor hypoxia. In the ideal situation, clinical PET imaging should be correlated with tumor pathological specimens (37), to evaluate the threshold to define tumor hypoxia.

For future dose re-distribution studies it is of importance to gain insight into the spatiotemporal stability of the PET tracer. A rigid registration was performed to compare the $\left[{ }^{18} \mathrm{~F}\right] \mathrm{HX} 4 \mathrm{PET}$ images at $2 \mathrm{~h}$ and $4 \mathrm{~h}$ p.i. Small errors in the registration can strongly affect the observed correlations negatively (38). In the current study PET scans were acquired in treatment position reducing the possibility for registration errors. Patients were freebreathing during PET-acquisitions, which might cause a blurring of the PET signal. However, this will not affect the registration, since breathing motion is the same for both acquisitions. By using a careful scan procedure and image registration a good spatio-temporal stability was found. 
Limiting the acquisition time is beneficial for practical reasons, to reduce patient movement and to increase patient comfort. As expected, image noise increases when reducing the acquisition time. This results in a significant change in CNR and SUV $V_{\max }$ for all reduced acquisition times. However $\mathrm{TBR}_{\max }$ and HF are only significantly influenced when acquisition time is reduced to $5 \mathrm{~min}$. This is in agreement with the results previously showed by Boellaard et al. (39), that SUV $V_{\text {max }}$ is more sensitive to image noise than SUV ratios like TBR. For this reason SUV $\mathrm{max}_{\text {ma }}$ is not be the best parameter to evaluate the uptake of hypoxia makers. A trade-off has to be made between image noise and practical issues, nonetheless, the acquisition-time for $\left[{ }^{18} \mathrm{~F}\right] \mathrm{HX} 4$ PET imaging at $4 \mathrm{~h}$ p.i. should be at least $10 \mathrm{~min}$, with the current administered activity and a modern Time-of-Flight PET/CT scanner. This provides the opportunity to acquire more bed positions, capturing the entire thorax in a 30 min time frame. Another option is to reduce the amount of injected activity, which will have a similar effect on image noise as a reduction of acquisition time.

In conclusion, significant hypoxia was observed in $72 \%$ of the NSCLC target lesions ( $80 \%$ of primary tumors and $60 \%$ of the involved lymph nodes). The heterogeneous $\left[{ }^{18} \mathrm{~F}\right] \mathrm{HX} 4$ uptake pattern was stable between $2 \mathrm{~h}$ and $4 \mathrm{~h}$ p.i., however the $\mathrm{TBR}_{\max }$ increased over time, suggesting that imaging at $4 \mathrm{~h}$ p.i. is better to reach the highest contrast in $\left[{ }^{18} \mathrm{~F}\right] \mathrm{HX} 4$ PET images. [ ${ }^{18} \mathrm{~F}$ ]HX4 PET acquisition time can be reduced to 10 minwithout significant effects on $\mathrm{TBR}_{\max }$ and HF.

\section{ACKNOWLEDGEMENTS}

The authors like to thank the patients who agreed to participate and R. Franssen and C. Urbach from the Department of Nuclear Medicine MUMC for their contribution to the data acquisition. We acknowledge financial support from the CTMM framework (AIRFORCE project), EU 6th and 7th framework program (ARTFORCE and METOXIA program), Interreg (www.eurocat.info), STW (DuCAT), the Kankeronderzoekfonds Limburg from the Health Foundation Limburg and the Dutch Cancer Society (KWF UM 2011-5020, KWF UM 2009-4454, KWF MAC 2011-4970).

\section{SUPPLEMENTARY DATA}




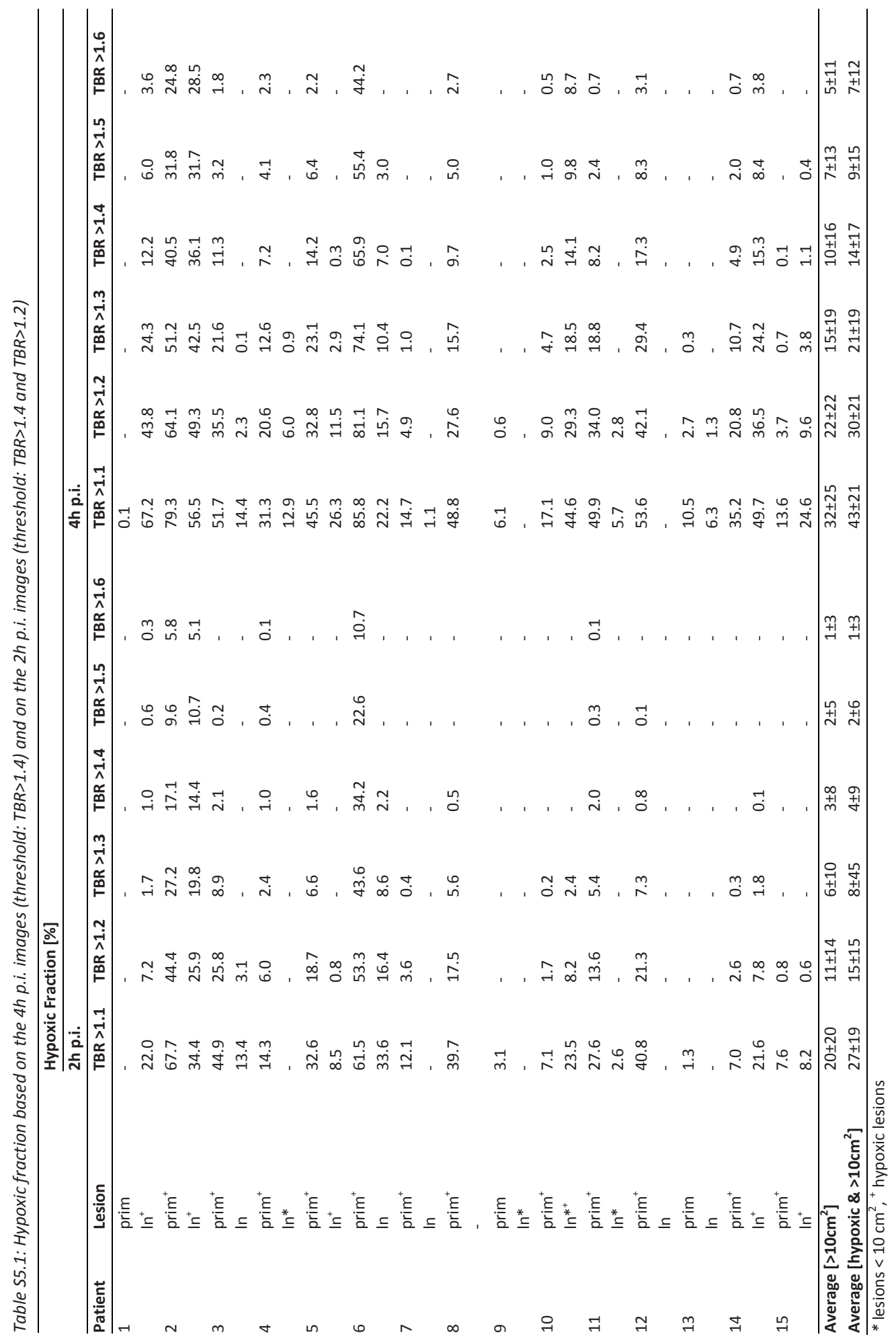


Table S5.2: DICE similarity coefficient for the high volume uptake regions in PET2h and PET4h, based on high percentiles and hypoxic fraction (HF). Pearson correltion coefficient for the voxel wise comparison within GTV.

\begin{tabular}{|c|c|c|c|c|c|c|c|c|c|}
\hline \multirow[t]{3}{*}{ Patient } & \multirow[t]{3}{*}{ Lesion } & \multicolumn{6}{|c|}{ Volume based } & \multicolumn{2}{|c|}{ Voxel Based } \\
\hline & & \multicolumn{5}{|c|}{ DICE [percentile] } & \multirow{2}{*}{ DICE [HF] } & \multirow[b]{2}{*}{$\mathbf{R}$} & \multirow[b]{2}{*}{ Slope } \\
\hline & & 50 & 60 & 70 & 80 & 90 & & & \\
\hline \multirow[t]{2}{*}{1} & prim & 0.89 & 0.86 & 0.82 & 0.79 & 0.66 & - & 0.94 & 0.73 \\
\hline & $\ln ^{+}$ & 0.76 & 0.72 & 0.68 & 0.61 & 0.52 & 0.56 & 0.71 & 0.74 \\
\hline \multirow[t]{2}{*}{2} & $\operatorname{prim}^{+}$ & 0.80 & 0.78 & 0.73 & 0.66 & 0.58 & 0.79 & 0.75 & 0.83 \\
\hline & $\ln ^{+}$ & 0.83 & 0.83 & 0.85 & 0.82 & 0.53 & 0.84 & 0.86 & 0.94 \\
\hline \multirow[t]{2}{*}{3} & $\operatorname{prim}^{+}$ & 0.72 & 0.68 & 0.61 & 0.59 & 0.48 & 0.48 & 0.58 & 0.47 \\
\hline & In & 0.74 & 0.62 & 0.51 & 0.37 & 0.25 & - & 0.67 & 0.67 \\
\hline \multirow[t]{2}{*}{4} & $\operatorname{prim}^{+}$ & 0.87 & 0.81 & 0.73 & 0.64 & 0.57 & 0.55 & 0.94 & 0.76 \\
\hline & $\ln *$ & 0.71 & 0.70 & 0.51 & 0.35 & 0.25 & - & 0.66 & 0.83 \\
\hline \multirow[t]{2}{*}{5} & $\operatorname{prim}^{+}$ & 0.78 & 0.74 & 0.70 & 0.63 & 0.54 & 0.59 & 0.76 & 0.71 \\
\hline & $\ln ^{+}$ & 0.88 & 0.85 & 0.79 & 0.68 & 0.44 & - & 0.85 & 0.66 \\
\hline \multirow[t]{2}{*}{6} & $\operatorname{prim}^{+}$ & 0.78 & 0.76 & 0.73 & 0.62 & 0.39 & 0.98 & 0.79 & 0.74 \\
\hline & $\ln ^{+}$ & 0.71 & 0.75 & 0.75 & 0.67 & 0.52 & 0.38 & 0.69 & 0.77 \\
\hline \multirow[t]{2}{*}{7} & $\operatorname{prim}^{+}$ & 0.76 & 0.68 & 0.60 & 0.51 & 0.45 & - & 0.79 & 0.47 \\
\hline & $\ln$ & 0.61 & 0.56 & 0.53 & 0.49 & 0.39 & - & 0.43 & 0.24 \\
\hline \multirow[t]{2}{*}{8} & $\operatorname{prim}^{+}$ & 0.81 & 0.79 & 0.74 & 0.66 & 0.59 & 0.59 & 0.79 & 0.94 \\
\hline & - & & & & & & & & \\
\hline \multirow[t]{2}{*}{9} & prim & 0.84 & 0.77 & 0.66 & 0.53 & 0.32 & - & 0.84 & 0.61 \\
\hline & $\ln *$ & 0.51 & 0.38 & 0.06 & 0.00 & 0.00 & - & -0.03 & -0.02 \\
\hline \multirow[t]{2}{*}{10} & $\operatorname{prim}^{+}$ & 0.81 & 0.77 & 0.73 & 0.68 & 0.60 & - & 0.84 & 0.63 \\
\hline & $\ln *^{+}$ & 0.80 & 0.84 & 0.79 & 0.72 & 0.67 & 0.62 & 0.82 & 1.00 \\
\hline \multirow[t]{2}{*}{11} & $\operatorname{prim}^{+}$ & 0.75 & 0.69 & 0.62 & 0.52 & 0.43 & 0.39 & 0.64 & 0.57 \\
\hline & $\ln *$ & 0.62 & 0.55 & 0.53 & 0.48 & 0.45 & - & 0.39 & 0.48 \\
\hline \multirow[t]{2}{*}{12} & $\operatorname{prim}^{+}$ & 0.89 & 0.85 & 0.80 & 0.75 & 0.59 & 0.72 & 0.93 & 0.99 \\
\hline & $\ln$ & 0.89 & 0.84 & 0.87 & 0.73 & 0.77 & - & 0.91 & 0.77 \\
\hline \multirow[t]{2}{*}{13} & prim & 0.87 & 0.78 & 0.67 & 0.67 & 0.63 & - & 0.89 & 0.73 \\
\hline & $\ln$ & 0.75 & 0.68 & 0.63 & 0.60 & 0.46 & - & 0.82 & 0.65 \\
\hline \multirow[t]{2}{*}{14} & $\operatorname{prim}^{+}$ & 0.74 & 0.64 & 0.53 & 0.40 & 0.31 & - & 0.74 & 0.65 \\
\hline & $\ln ^{+}$ & 0.74 & 0.68 & 0.61 & 0.48 & 0.35 & 0.44 & 0.61 & 0.66 \\
\hline \multirow[t]{2}{*}{15} & $\operatorname{prim}^{+}$ & 0.82 & 0.77 & 0.67 & 0.53 & 0.29 & - & 0.76 & 0.70 \\
\hline & $\ln ^{+}$ & 0.85 & 0.78 & 0.69 & 0.58 & 0.38 & - & 0.81 & 0.79 \\
\hline \multirow{2}{*}{\multicolumn{2}{|c|}{ Average $\left[>10 \mathrm{~cm}^{3}\right]$}} & $0.79 \pm$ & $0.75 \pm$ & $0.69 \pm$ & $0.61 \pm$ & $0.48 \pm$ & $0.61 \pm$ & $0.77 \pm$ & $0.70 \pm$ \\
\hline & & 0.07 & 0.08 & 0.10 & 0.11 & 0.13 & 0.19 & 0.12 & 0.16 \\
\hline \multirow{2}{*}{\multicolumn{2}{|c|}{$\begin{array}{l}\text { Average [hypoxic \& } \\
>10 \mathrm{~cm}^{3} \text { ] }\end{array}$}} & $0.79 \pm$ & $0.75 \pm$ & $0.70 \pm$ & $0.61 \pm$ & $0.48 \pm$ & $0.61 \pm$ & $0.77 \pm$ & $0.72 \pm$ \\
\hline & & 0.06 & 0.06 & 0.08 & 0.10 & 0.10 & 0.19 & 0.10 & 0.15 \\
\hline
\end{tabular}

* lesions $<10 \mathrm{~cm}^{2},{ }^{+}$hypoxic lesions, - HF smaller than $5 \%$ 


\section{REFERENCES}

1. van Baardwijk A, Reymen B, Wanders S, Borger J, Ollers $M$, Dingemans $A M$, et al. Mature results of a phase II trial on individualised accelerated radiotherapy based on normal tissue constraints in concurrent chemo-radiation for stage III non-small cell lung cancer. Eur J Cancer. 2012;48:2339-46.

2. Wouters BG, van den Beucken T, Magagnin MG, Lambin P, Koumenis C. Targeting hypoxia tolerance in cancer. Drug Resist Updat. 2004;7:25-40.

3. Lambin P, van Stiphout RG, Starmans MH, Rios-Velazquez E, Nalbantov G, Aerts HJ, et al. Predicting outcomes in radiation oncology--multifactorial decision support systems. Nat Rev Clin Oncol. 2013;10:27-40.

4. Overgaard J. Hypoxic modification of radiotherapy in squamous cell carcinoma of the head and neck - A systematic review and meta-analysis. Radiother Oncol. 2011;100:22-32.

5. Mortensen LS, Johansen J, Kallehauge J, Primdahl H, Busk M, Lassen P, et al. FAZA $\mathrm{PET} / \mathrm{CT}$ hypoxia imaging in patients with squamous cell carcinoma of the head and neck treated with radiotherapy: results from the DAHANCA 24 trial. Radiother Oncol. 2012;105:14-20.

6. Eschmann SM, Paulsen F, Reimold M, Dittmann H, Welz S, Reischl G, et al. Prognostic impact of hypoxia imaging with 18F-misonidazole PET in non-small cell lung cancer and head and neck cancer before radiotherapy. J Nucl Med. 2005;46:253-60.

7. Gagel B, Reinartz P, Demirel C, Kaiser HJ, Zimny M, Piroth M, et al. [18F] fluoromisonidazole and [18F] fluorodeoxyglucose positron emission tomography in response evaluation after chemo-/radiotherapy of non-small-cell lung cancer: a feasibility study. BMC Cancer. 2006;6:51.

8. Vera P, Bohn P, Edet-Sanson A, Salles A, Hapdey S, Gardin I, et al. Simultaneous positron emission tomography (PET) assessment of metabolism with (1)(8)F-fluoro-2deoxy-d-glucose (FDG), proliferation with (1)(8)F-fluoro-thymidine (FLT), and hypoxia with (1)(8)fluoro-misonidazole (F-miso) before and during radiotherapy in patients with non-small-cell lung cancer (NSCLC): a pilot study. Radiother Oncol. 2011;98:109-16.

9. Bittner MI, Wiedenmann N, Bucher S, Hentschel M, Mix M, Weber WA, et al. Exploratory geographical analysis of hypoxic subvolumes using F-MISO-PET imaging in patients with head and neck cancer in the course of primary chemoradiotherapy. Radiother Oncol. 2013.

10. Bollineni VR, Kerner GS, Pruim J, Steenbakkers RJ, Wiegman EM, Koole MJ, et al. PET Imaging of Tumor Hypoxia Using 18F-Fluoroazomycin Arabinoside in Stage III-IV NonSmall Cell Lung Cancer Patients. J Nucl Med. 2013.

11. Zips D, Boke S, Kroeber T, Meinzer A, Bruchner K, Thames HD, et al. Prognostic Value of Radiobiological Hypoxia during Fractionated Irradiation for Local Tumor Control. Strahlenther Onkol. 2011;187:306-10.

12. Lambin P, Petit SF, Aerts HJ, van Elmpt WJ, Oberije CJ, Starmans MH, et al. The ESTRO Breur Lecture 2009. From population to voxel-based radiotherapy: exploiting intratumour and intra-organ heterogeneity for advanced treatment of non-small cell lung cancer. Radiother Oncol. 2010;96:145-52.

13. Hendrickson K, Phillips M, Smith W, Peterson L, Krohn K, Rajendran J. Hypoxia imaging with [F-18] FMISO-PET in head and neck cancer: potential for guiding 
intensity modulated radiation therapy in overcoming hypoxia-induced treatment resistance. Radiother Oncol. 2011;101:369-75.

14. Thorwarth D, Eschmann SM, Paulsen F, Alber M. Hypoxia dose painting by numbers: a planning study. Int J Radiat Oncol Biol Phys. 2007;68:291-300.

15. van Elmpt W, De Ruysscher D, van der Salm A, Lakeman A, van der Stoep J, Emans D, et al. The PET-boost randomised phase II dose-escalation trial in non-small cell lung cancer. Radiother Oncol. 2012;104:67-71.

16. Bollineni VR, Wiegman EM, Pruim J, Groen HJ, Langendijk JA. Hypoxia imaging using Positron Emission Tomography in non-small cell lung cancer: implications for radiotherapy. Cancer Treat Rev. 2012;38:1027-32.

17. Horsman MR, Mortensen LS, Petersen JB, Busk M, Overgaard J. Imaging hypoxia to improve radiotherapy outcome. Nat Rev Clin Oncol. 2012;9:674-87.

18. Dubois LJ, Lieuwes NG, Janssen MH, Peeters WJ, Windhorst AD, Walsh JC, et al. Preclinical evaluation and validation of [18F]HX4, a promising hypoxia marker for PET imaging. Proc Natl Acad Sci U S A. 2011;108:14620-5.

19. van Loon J, Janssen $M H$, Ollers $M$, Aerts HJ, Dubois L, Hochstenbag $M$, et al. PET imaging of hypoxia using [18F]HX4: a phase I trial. Eur J Nucl Med Mol Imaging. 2010;37:1663-8.

20. Doss $M$, Zhang JJ, Belanger MJ, Stubbs JB, Hostetler ED, Alpaugh $K$, et al. Biodistribution and radiation dosimetry of the hypoxia marker 18F-HX4 in monkeys and humans determined by using whole-body PET/CT. Nucl Med Commun. 2010;31:1016-24.

21. Chen L, Zhang Z, Kolb HC, Walsh JC, Zhang J, Guan Y. (1)(8)F-HX4 hypoxia imaging with PET/CT in head and neck cancer: a comparison with (1)(8)F-FMISO. Nucl Med Commun. 2012;33:1096-102.

22. Rasey JS, Koh WJ, Evans ML, Peterson LM, Lewellen TK, Graham MM, et al. Quantifying regional hypoxia in human tumors with positron emission tomography of [18F]fluoromisonidazole: a pretherapy study of 37 patients. Int J Radiat Oncol Biol Phys. 1996;36:417-28.

23. Koh WJ, Bergman KS, Rasey JS, Peterson LM, Evans ML, Graham MM, et al. Evaluation of oxygenation status during fractionated radiotherapy in human nonsmall cell lung cancers using [F-18]fluoromisonidazole positron emission tomography. Int J Radiat Oncol Biol Phys. 1995;33:391-8.

24. Dubois L, Landuyt W, Haustermans K, Dupont P, Bormans G, Vermaelen P, et al. Evaluation of hypoxia in an experimental rat tumour model by [(18)F]fluoromisonidazole PET and immunohistochemistry. Br J Cancer. 2004;91:1947-54.

25. Gray AJ, Dische S, Adams GE, Flockhart IR, Foster JL. Clinical testing of the radiosensitiser Ro-07-0582. I. Dose tolerance, serum and tumour concentrations. Clin Radiol. 1976;27:151-7.

26. Saunders ME, Dische S, Anderson P, Flockhart IR. The neurotoxicity of misonidazole and its relationship to dose, half-life and concentration in the serum. $\mathrm{Br} J$ Cancer Suppl. 1978;3:268-70.

27. Abolmaali N, Haase R, Koch A, Zips D, Steinbach J, Baumann M, et al. Two or four hour [(1)(8)F]FMISO-PET in HNSCC. When is the contrast best? Nuklearmedizin. 2011;50:22-7. 
28. Hu M, Xing L, Mu D, Yang W, Yang G, Kong L, et al. Hypoxia Imaging With 18FFluoroerythronitroimidazole Integrated PET/CT and Immunohistochemical Studies in Non-Small Cell Lung Cancer. Clin Nucl Med. 2013;38:591-6.

29. Li L, Hu M, Zhu H, Zhao W, Yang G, Yu J. Comparison of 18F-Fluoroerythronitroimidazole and 18F-fluorodeoxyglucose positron emission tomography and prognostic value in locally advanced non-small-cell lung cancer. Clin Lung Cancer. 2010;11:335-40.

30. Cherk MH, Foo SS, Poon AM, Knight SR, Murone C, Papenfuss AT, et al. Lack of correlation of hypoxic cell fraction and angiogenesis with glucose metabolic rate in non-small cell lung cancer assessed by $18 \mathrm{~F}-$ Fluoromisonidazole and 18F-FDG PET. J Nucl Med. 2006;47:1921-6.

31. Trinkaus ME, Blum R, Rischin D, Callahan J, Bressel M, Segard T, et al. Imaging of hypoxia with (18) F-FAZA PET in patients with locally advanced non-small cell lung cancer treated with definitive chemoradiotherapy. J Med Imaging Radiat Oncol. 2013;57:475-81.

32. Postema EJ, McEwan AJ, Riauka TA, Kumar P, Richmond DA, Abrams DN, et al. Initial results of hypoxia imaging using 1-alpha-D: -(5-deoxy-5-[18F]-fluoroarabinofuranosyl)-2-nitroimidazole ( 18F-FAZA). Eur J Nucl Med Mol Imaging. 2009;36:1565-73.

33. Grau C, Overgaard J. Effect of cancer chemotherapy on the hypoxic fraction of a solid tumor measured using a local tumor control assay. Radiother Oncol. 1988;13:301-9.

34. Nehmeh SA, Lee NY, Schroder H, Squire O, Zanzonico PB, Erdi YE, et al. Reproducibility of intratumor distribution of (18)F-fluoromisonidazole in head and neck cancer. Int J Radiat Oncol Biol Phys. 2008;70:235-42.

35. Dirix P, Vandecaveye V, De Keyzer F, Stroobants S, Hermans R, Nuyts S. Dose painting in radiotherapy for head and neck squamous cell carcinoma: value of repeated functional imaging with (18)F-FDG PET, (18)F-fluoromisonidazole PET, diffusionweighted MRI, and dynamic contrast-enhanced MRI. J Nucl Med. 2009;50:1020-7.

36. Okamoto S, Shiga T, Yasuda K, Ito YM, Magota K, Kasai K, et al. High reproducibility of tumor hypoxia evaluated by 18F-fluoromisonidazole PET for head and neck cancer. J Nucl Med. 2013;54:201-7.

37. van Baardwijk A, Dooms C, van Suylen RJ, Verbeken E, Hochstenbag M, DehingOberije $C$, et al. The maximum uptake of (18)F-deoxyglucose on positron emission tomography scan correlates with survival, hypoxia inducible factor-1alpha and GLUT1 in non-small cell lung cancer. Eur J Cancer. 2007;43:1392-8.

38. Nyflot MJ, Harari PM, Yip S, Perlman SB, Jeraj R. Correlation of PET images of metabolism, proliferation and hypoxia to characterize tumor phenotype in patients with cancer of the oropharynx. Radiother Oncol. 2012;105:36-40.

39. Boellaard R, Krak NC, Hoekstra OS, Lammertsma AA. Effects of noise, image resolution, and $\mathrm{ROI}$ definition on the accuracy of standard uptake values: a simulation study. J Nucl Med. 2004;45:1519-27. 

CLINICAL POTENTIAL AND THERAPEUTIC TARGETS 



\section{CHAPTER}

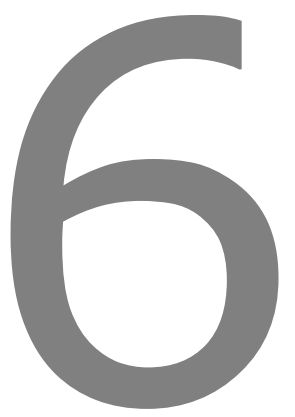

In vivo quantification of hypoxic and metabolic status of NSCLC using $\left[{ }^{18} \mathrm{~F}\right] \mathrm{HX} 4$ and $\left[{ }^{18} \mathrm{~F}\right] \mathrm{FDG} \mathrm{PET} / \mathrm{CT}$ imaging

Catharina M.L. Zegers, Wouter van Elmpt, Bart Reymen, Aniek J.G. Even, Esther G.C. Troost, Michel C. Öllers, Frank J.P. Hoebers, Ruud M.A. Houben, Jonas Eriksson, Albert D.Windhorst, Felix M. Mottaghy, Dirk De Ruysscher, Philippe Lambin 


\section{ABSTRACT}

Purpose: Increased tumor metabolism and hypoxia are related to poor prognosis in solid tumors, including non-small cell lung cancer (NSCLC). PET imaging is a non-invasive technique which is frequently used to visualize and quantify tumor metabolism and hypoxia. The aim of this study was to perform an extensive comparison of tumor metabolism using $2\left[{ }^{18}\right.$ F fluoro-2-deoxy-D-glucose (FDG)-PET and hypoxia using HX4-PET imaging.

Experimental Design: FDG- and HX4-PET/CT images of 25 NSCLC patients were coregistered. At a global tumor level, HX4 and FDG parameters were extracted from the gross tumor volume. The HX4 high-fraction (HX4-HF) and high-volume (HX4-HV) were defined using a tumor-to-blood ratio $>1.4$. For FDG high-fraction (FDG-HF) and high-volume (FDG$\mathrm{HV}$ ) a standardized uptake value (SUV) $>50 \%$ of $\mathrm{SUV}_{\max }$ was used. We evaluated the spatial correlation between HX4 and FDG uptake within the tumor, to quantify the (mis)match between volumes with a high FDG and high HX4 uptake.

Results: At a tumor-level, significant correlations were observed between FDG and HX4 parameters. For the primary GTV, the HX4-HF was three times smaller compared to the FDG-HF. In $53 \%$ of the primary lesions, less than $1 \mathrm{~cm}^{3}$ of the HX4-HV was outside the FDG$\mathrm{HV}$; for $37 \%$ this volume was 1.9 to $12 \mathrm{~cm}^{3}$. Remarkably, a distinct uptake pattern was observed in $11 \%$, with large hypoxic volumes localized outside the FDG-HV.

Conclusion: Hypoxic tumor volumes are smaller than metabolic active volumes. Approximately half of the lesions showed a good spatial correlation between the PET tracers. In the other cases, a (partial) mismatch was observed. The addition of HX4-PET imaging has the potential to individualize patient treatment. 


\section{INTRODUCTION}

Lung cancer has the highest death rate among leading cancer types (1). Standard treatment for advanced stage non-small cell lung cancer (NSCLC) is the combination of radiotherapy and chemotherapy, administered either sequentially or concurrently (2). Tumor cell hypoxia has a negative impact on cancer treatment effectiveness, it promotes resistance to radiotherapy and chemotherapy, increases the metastatic potential, and is therefore related to a poor prognosis (3-5). Tumor hypoxia is present in the majority of NSCLCs, which can be visualized and quantified using functional PET imaging with radiolabeled 2-nitroimidazoles (6, 7). 3-[ ${ }^{18}$ F]fluoro-2-(4-((2-nitro- $1 \mathrm{H}$-imidazol-1-yl)methyl)- $1 \mathrm{H}$ 1,2,3-triazol-1-yl)propan-1-ol $\left(\left[{ }^{18} \mathrm{~F}\right] \mathrm{HX} 4\right)$ is a relatively new nitroimidazole with attractive pharmacokinetic properties that has successfully completed preclinical and clinical testing (8-10).

In standard clinical practice, a combination of anatomic computed tomography (CT) and functional $2\left[{ }^{18} \mathrm{~F}\right]$ fluoro-2-deoxy-D-glucose (FDG) PET imaging is frequently used to visualize, detect and stage malignancies. In addition, FDG-PET can be used to identify subvolumes with a high metabolism, which are more susceptible to local recurrence after (chemo)radiotherapy $(11,12)$. Aerts and colleagues showed that the residual tumor volume after radiotherapy is mainly located within the pre-radiotherapy high FDG-uptake volume. However, $30 \%$ of the residual volume did not correspond to the high FDG volume $(11,13)$. This may be caused by tumor regrowth in pre-radiotherapy hypoxic tumor subvolumes located outside the high FDG volume. Therefore, it is of great interest to investigate the correlation between both unfavorable biological features (high tumor metabolism, hypoxia) because they can be used to predict treatment outcome. In addition, imaging-derived tumor features have the potential to guide treatment with hypoxic modifiers or radiotherapy dose-painting (14-16). The uptake of FDG in the cell is dependent on the over-expression of glucose transporters (GLUT), which can be upregulated in the absence of oxygen, through the HIF1 $\alpha$-mediated pathway (17). This may suggest a possible overlap between volumes of high FDG uptake and tumor hypoxia, even though they represent different biological properties of tumors.

The aim of this study was to perform an extensive comparison of tumor metabolism, using FDG, and hypoxia, using HX4, to fully characterize the relationship between both PET tracers on a global tumor and voxel level for primary NSCLC and the regional lymph node metastases. 


\section{MATERIALS AND METHODS}

\section{Patients}

FDG- and HX4-PET/CT images of 25 NSCLC patients (17 male and 8 female) were acquired before the start of external beam radiotherapy. The average age of the patients was 63 years (range, 40-82 years). Tumor stage ranged from IIB-IV, pathology being adenocarcinoma $(N=13)$, squamous cell carcinoma (SCC; $N=5)$, and large cell carcinoma $(N=7)$. Patients were treated with radical radiotherapy $(N=3)$ or chemoradiation $(N=22)$, with the majority of patients receiving at least one cycle of chemotherapy before PET imaging and before the start of radiotherapy (Table S6.1). PET data were acquired in the translational research part of two phase II trials [PET-Boost, NCT01024829 (18); Nitroglycerin (NCT01210378)], both having identical PET imaging procedures. The clinical trials were approved by the appropriate medical ethical review committee, and all patients provided written informed consent before study entry.

\section{PET/CT imaging}

HX4 was produced as described in previous publications (8-10, 19). After intravenous administration of $429 \pm 57 \mathrm{MBq} \mathrm{HX} 4, \mathrm{PET} / \mathrm{CT}$ imaging was performed at $4 \mathrm{~h}$ postinjection (p.i.) for 20 to 30 minutes in a single bed position centered around the primary tumor. HX4-PET/CT images were acquired on a Gemini TF 64 scanner (Philips Healthcare, Best, the Netherlands), with a spatial resolution of approximately $5 \mathrm{~mm}$ FWHM. We performed CT-based attenuation correction and scatter correction (SS-SIMUL), and reconstructed PET images using 3D ordered subset iterative time-of-flight reconstruction technique (BLOBOS-TF) with three iterations and 33 subsets in a $144 \times 144$ matrix and voxel sizes of $4 \times 4$ $\mathrm{mm}$.

The injected activity of FDG was based on the patient's body weight according to the national guidelines (20). PET/CT imaging was performed one hour after intravenous administration of FDG. FDG PET/CT scans were acquired using a Biograph 40 PET/CT scanner (Siemens Healthcare, Erlangen, Germany).Scatter and attenuation corrections were applied. PET images were reconstructed using OSEM 2D (Ordered Subset Expectation Maximization, four iterations, eight subsets) and a Gaussian filter of $5 \mathrm{~mm}$. A respiratory correlated CT was performed, with the mid-ventilation scan selected for the attenuation correction and fusion with the FDG PET.

HX4- and FDG-PET/CT scans were acquired in the same week for all except 1 patient. The median interval between both PET scans was 3 days (range: 1-14 days). No interventions (e.g. radiotherapy or chemotherapy) were performed between the FDG- and HX4-PET scans. Both scans were acquired with the patient positioned in radiotherapy position, on a flat tabletop using a laser alignment system with arms in an arm-support positioned above the head. 


\section{Analysis}

For all patients, gross tumor volumes (GTV), including the primary lesion $\left(G V_{\text {prim }}\right)$ and involved lymph nodes $\left(G_{T V}\right.$ In $)$, were defined on the FDG-PET/CT scan by two experienced radiation oncologists in consensus. $\mathrm{GTV}_{\text {prim }}$ and $\mathrm{GTV}_{\mathrm{In}}$ were analyzed separately. Lesions with a size $<5 \mathrm{~cm}^{3}$ were excluded due to potential partial volume effects.

The FDG-PET/CT was rigidly registered to the HX4-PET/CT using registration software developed in-house. The rigid transformation was determined by the registration of the FDG-CT to the HX4-CT; the same transformation was subsequently applied to the FDG-PET scan and the GTVs. A volume of interest in the aorta was defined as background region.

The maximum and mean standardized uptake values $\left(S U V_{\max }\right.$ and $\left.S U V_{\text {mean }}\right)$, corrected for body weight were determined within the GTV for both FDG- and HX4-PET. For the HX4$P E T$, calculations were made of the maximum tumor-to-blood ratio $\left(T B R_{\max }\right)$, defined as the SUV $V_{\text {max }}$ in the tumor divided by the SUV mean in the aorta, the HX4 high-fraction (HX4$\mathrm{HF}$ ) and HX4 high-volume (HX4-HV), both defined as the fraction/volume of the GTV with a TBR >1.4. For the FDG PET, calculations were made of the FDG high-fraction (FDG-HF) and FDG high-volume (FDG-HV) based on the PET-Boost trial strategy, using the GTV volume with an SUV above $50 \%$ of the $\operatorname{SUV}_{\max }(18)$.

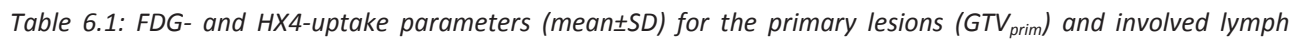
nodes $\left(G T V_{l n}\right)$.

\begin{tabular}{lll}
\hline & $\begin{array}{l}\text { GTVprim } \\
\text { average (range) } \\
\mathrm{N}\end{array}$ & $\begin{array}{l}\text { GTVIn } \\
\text { average (range) } \\
19\end{array}$ \\
\hline Volume GTV $^{25}$ & $57 \pm 72 \mathrm{~cm}^{3}\left(7-254 \mathrm{~cm}^{3}\right)$ \\
FDG-SUV & $3.3 \pm 2.5(1.7-10.9)$ & $3.9 \pm 1.5(1.3-6.7)$ \\
FDG-SUV & $13.3 \pm 6.6(3.5-30.4)$ & $9.3 \pm 4.0(2.4-18.5)$ \\
FDG-HF & $41 \pm 21 \%(10-85 \%)$ & $49 \pm 22 \%(20-92 \%)$ \\
FDG-HV & $36 \pm 33 \mathrm{~cm}^{3}\left(5-123 \mathrm{~cm}^{3}\right)$ & $29 \pm 40 \mathrm{~cm}^{3}\left(2-139 \mathrm{~cm}^{3}\right)$ \\
HX4-SUV & $0.8 \pm 0.3(0.4-1.2)$ & $0.7 \pm 0.2(0.4-1.0)$ \\
HX4-SUV & $1.3 \pm 0.4(0.6-2.1)$ & $1.1 \pm 0.4(0.5-1.8)$ \\
HX4-TBR & $1.8 \pm 0.6(0.9-3.5)$ & $1.7 \pm 0.5(1.1-2.8)$ \\
HX4-HF & $14 \pm 15 \%(0-49 \%)$ & $7 \pm 10 \%(0-37 \%)$ \\
HX4-HV & $18 \pm 30 \mathrm{~cm}^{3}\left(0-131 \mathrm{~cm}^{3}\right)$ & $7 \pm 13 \mathrm{~cm}^{3}\left(0-46 \mathrm{~cm}^{3}\right)$ \\
\hline
\end{tabular}

This classification for defining HX4-HVand FDG-HV, as a fraction of the total GTV, was used to sub-divide regions of a tumor into four classes: (i) FDG-low and HX4-low, (ii) FDG-high and HX4-low, (iii) FDG-low and HX4-high, and (iv) FDG-high and HX4-high. To evaluate the effect of the threshold definition on tumor subdivision, a calculation was made of the average distribution using alternative thresholds. The HX4 threshold varied from TBR $>1.3$ to TBR $>1.5$; the FDG threshold ranged from SUV $>30 \%$ to SUV $>70 \%$ of SUV $_{\max }$. 
A visual and voxel-wise comparison of the FDG and HX4 uptake within the GTV was performed to compare spatial uptake patterns in the primary lesions. On the basis of the voxel-wise analysis we separated lesions into three groups. First, in lesions showing a high correlation between the FDG and HX4 uptake, the hypoxic volume was entirely within the high metabolic volume. Second, in lesions showing a moderate correlation between the FDG and HX4 uptake, there was only a partial overlap between the HX4-HV and the FDGHV. Third, in lesions showing a different uptake pattern between the two tracers, there were two distinct regions of FDG-HV and HX4-HV.

\section{Statistical analysis}

Mean \pm 1 SD were reported for all parameters. Linear and multiple linear regressions were performed to correlate the GTV-based parameters (SUV $\left.{ }_{\text {max }}, S_{U} V_{\text {mean }}, T B R, H F, H V\right)$ and to quantify the voxel-wise comparison of the FDG and HX4 uptake. Pearson correlation coefficients were calculated. A Pvalue of $<0.05$ was assumed to be statistically significant.

Table 6.2: Pearson's correlation coefficient (R) and corresponding Pvalues of GTV and $H X 4-P E T$

\begin{tabular}{|c|c|c|c|c|c|c|}
\hline & $\begin{array}{l}\text { Volume } \\
\text { GTV }_{\text {prim }}\end{array}$ & $\begin{array}{l}\text { HX4 - } \\
\text { SUV }_{\text {mean }}\end{array}$ & $\begin{array}{l}\text { HX4- } \\
\text { SUV }_{\max }\end{array}$ & $\begin{array}{l}\text { HX4- } \\
\text { TBR }_{\text {max }}\end{array}$ & $\begin{array}{l}\text { HX4- } \\
\text { HF }\end{array}$ & $\begin{array}{l}\text { HX4- } \\
\text { HV }\end{array}$ \\
\hline \multicolumn{7}{|c|}{ Volume $\mathrm{GTV}_{\text {prim }}$} \\
\hline $\mathrm{R}$ & - & 0.16 & 0.48 & 0.49 & 0.01 & 0.60 \\
\hline$P$ & - & 0.48 & 0.02 & 0.01 & 0.95 & $<0.01$ \\
\hline \multicolumn{7}{|c|}{ FDG-SUV ${ }_{\text {mean }}$} \\
\hline $\mathrm{R}$ & 0.13 & 0.52 & 0.58 & 0.46 & 0.44 & 0.52 \\
\hline$P$ & 0.54 & 0.01 & $<0.01$ & 0.02 & 0.03 & $<0.01$ \\
\hline \multicolumn{7}{|c|}{ FDG-SUV max $_{\max }$} \\
\hline $\mathrm{R}$ & 0.47 & 0.39 & 0.54 & 0.55 & 0.28 & 0.66 \\
\hline$P$ & 0.02 & 0.07 & $<0.01$ & $<0.01$ & 0.17 & $<0.001$ \\
\hline \multicolumn{7}{|c|}{ FDG-HF } \\
\hline$R$ & -0.47 & 0.20 & 0.02 & -0.14 & 0.31 & -0.18 \\
\hline$P$ & 0.02 & 0.36 & 0.93 & 0.49 & 0.13 & 0.40 \\
\hline \multicolumn{7}{|c|}{ FDG-HV } \\
\hline$R$ & 0.83 & 0.32 & 0.61 & 0.64 & 0.28 & 0.76 \\
\hline$P$ & $<0.0001$ & 0.14 & $<0.01$ & $<0.01$ & 0.18 & $<0.0001$ \\
\hline
\end{tabular}

\section{RESULTS}

\section{Overall correlation of FDG and HX4 parameters}

This study analyzed the overall FDG and HX4 uptake in the primary tumor and lymph nodes of 25 patients with NSCLC. All GTV prim $(N=25)$ and $19 \mathrm{GTV}_{\text {In }}$ were larger than $5 \mathrm{~cm}^{3}$ and all were used for the analysis. The average values of the GTV, FDG and HX4 parame- 
ters are shown in Table 6.1. The subclassification, based on tumor pathology, showed no significant differences for any of the FDG or HX4 parameters (examples are shown in Figure S6.1). The FDG-HV was larger than the HX4-HV in 24/25 GTV prim and in all $G_{T V}$. Potential correlations between FDG- and HX4-PET based parameters were investigated.

The correlation coefficients for the primary tumors are shown in Table 6.2. The majority of the FDG- and HX4-PET based parameters showed a significant correlation with the primary tumour volume. Note that the HX4-HV was significantly correlated with the tumor volume,

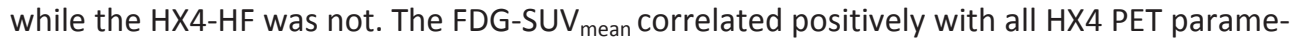
ters. The FDG-SUV ${ }_{\max }$ only showed a significant correlation with HX4-SUV $\mathrm{Hax}_{\max }(R=0.54 ; P$ $<0.01)$, HX4-TBR $(R=0.55 ; P<0.01)$ and HX4-HV $(R=0.66, P<0.001)$. The highest correlations were observed when comparing the FDG-HV with the HX4 based parameters: HX4$\operatorname{SUV}_{\text {max }}(R=0.63, P<0.01), \mathrm{HX} 4-\mathrm{TBR}(R=0.62, P<0.01)$, and HX4-HV $(R=0.76, P<0.0001)$. Two examples are shown in Figure 6.1. From Figure 6.1B one can appreciate that, although there is a correlation between FDG-SUV $V_{\max }$ and HX4-TBR $\mathrm{max}_{\text {, }}$ it is not possible to distinguish the non-hypoxic lesions by using only the FDG-SUV $\max$ parameter.
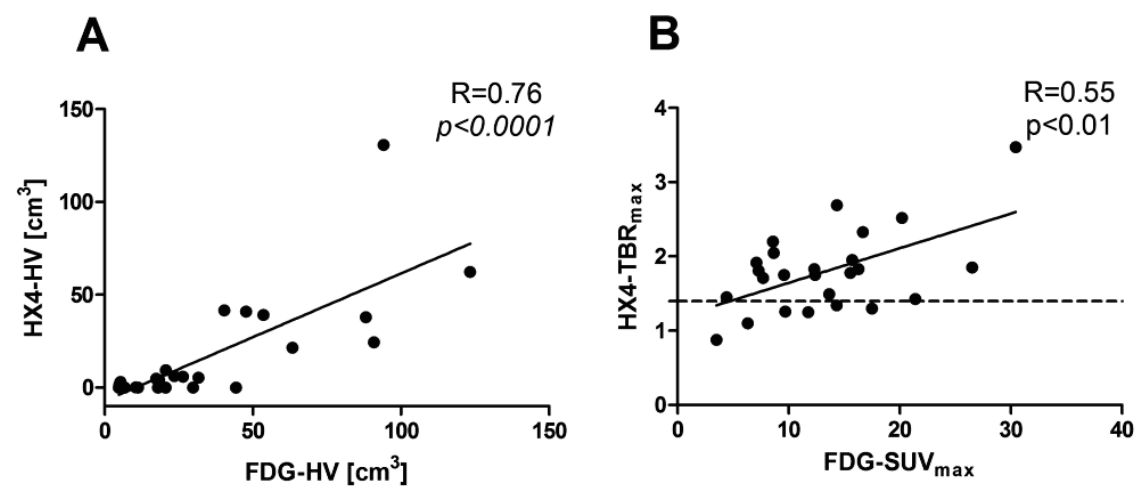

Figure 6.1: Comparison between FDG- and HX4-PET based parameters: A), FDG-HVversus HX4-HV and B), FDG$S U V_{\max }$ versus $H X 4-T B R$, the dashed line shows the threshold to define hypoxia $\left(H X 4-T B R_{\max }=1.4\right)$.

A multiple linear regression was performed in order to test the interaction between primary tumour volume and FDG parameters to predict the hypoxic volume. Using the parameters primary tumor volume and FDG-SUV max $_{\text {ax }}$ to predict HX4-HV, we observed a correlation coefficient of $0.74\left(R^{2}=0.55\right)$ with a significant contribution of both FDG$\operatorname{SUV}_{\max }(P<0.01)$ and primary tumor volume $(P=0.03)$. Adding the interaction term (FDG$\mathrm{SUV}_{\max } *$ primary tumor volume) to the model increases the correlation coefficient to 0.82 $\left(R^{2}=0.67\right)$.

For the involved lymph nodes $\mathrm{GTV}_{\text {In }}$ volume has a large effect on the correlation coefficients between the HX4 and FDG parameters (Table S6.2). The multiple linear regression using GTV In $_{\text {volume and FDG-SUV }}$ max to predict HX4-HV $(R=0.96)$ therefore only showed a significant contribution for the GTV $\operatorname{IV}_{\text {In }}$ volume $(P<0.001)$ and not for FDG-SUV $\max (P=0.26)$. 


\section{Average distribution of FDG and HX4 uptake}

The average distribution within the primary tumor based on the four previously predefined categories is shown in Table 6.3 and visualized in Figure 6.2A. On average, the FDG$\mathrm{HVis} 42 \pm 21 \%$ of the $\mathrm{GTV}_{\text {prim }}$, of which $10 \pm 12 \%$ is hypoxic. On average, $3 \%$ (range: $0 \%$ $31 \%$ ) of the GTVprim is hypoxic but outside the FDG-HV, representing $24 \%(3.2 \% / 13.6 \%)$ of the total hypoxic volume.

The effect of alternative thresholds on the average distribution of FDG and HX4 within the primary tumor is shown in Table S6.3 and visualized for two examples in Figures $6.2 \mathrm{~B}$ and 6.2C. This figure shows that the hypoxic percentage of the GTV (HX4-HF) outside the high FDG area (FDG-HF) is relatively stable.

Table 6.3: Average distribution of high and low HX4 and FDG uptake within the GTV prim.

\begin{tabular}{llll}
\hline Overlap between & $\begin{array}{l}\text { FDG-low } \\
\text { average (range) }\end{array}$ & $\begin{array}{l}\text { FDG-high } \\
\text { average (range) }\end{array}$ & GTV \\
\hline HX4-low & (i) $55.3 \pm 21.9 \%$ & (ii) $31.1 \pm 19.5 \%$ & $86.4 \pm 15.5$ \\
& $(8.5-89.8 \%)$ & $(9.8-84.3 \%)$ & $150.7-100 \%)$ \\
HX4-high & (iii) $3.2 \pm 6.5 \%$ & (iv) $10.4 \pm 12.2 \%$ & $(0-49.3 \%)$ \\
& $(0-31.0 \%)$ & $(0-43.4 \%)$ & $100 \%$ \\
GTV & $58.5 \pm 21.6 \%$ & $41.5 \pm 21.2 \%$ & \\
\hline
\end{tabular}

NOTE: Standard thresholds were used: TBR $>1.4(H X 4)$ and SUV $>50 \% S_{\text {max }}(F D G)$

A

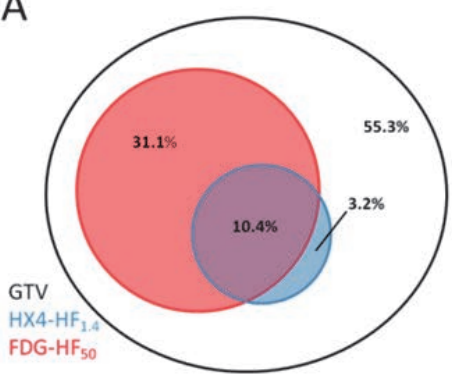

C

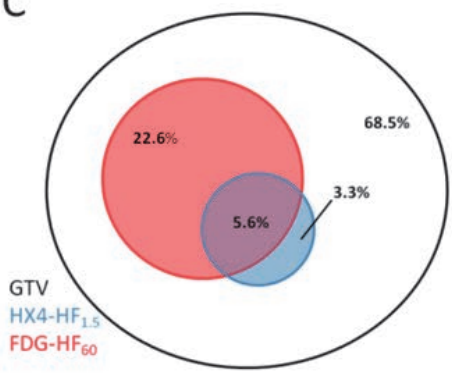

B

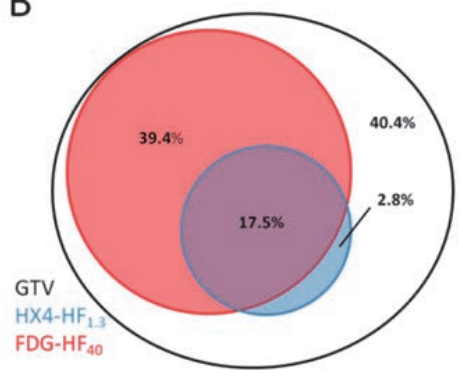

Figure 6.2: Visualization of the overlap between FDG-HF and HX4-HF. A), using the standard thresholds TBR $>1.4$

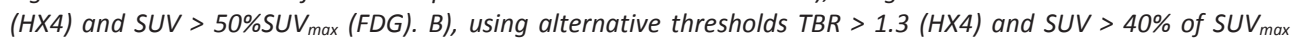
(FDG). C), using the alternative thresholds TBR $>1.5$ (HX4) and SUV $>60 \%$ of $S U V_{\max }$ (FDG). 


\section{Spatial correlation of FDG- and HX4-uptake patterns}

Tracer uptake above the background level in both PET scans is essential for comparing the overlap of FDG-HV and HX4-HV. All primary lesions showed FDG uptake with an SUV max $_{\text {> }}$ 3.5, however, only 19 out of 25 primary lesions expressed an HX4 uptake (TBR >1.4). These 19 lesions were selected for further analysis.

On the basis of the voxel-wise analysis, we observed that in 10 lesions, less than $1 \mathrm{~cm}^{3}$ of the HX4-HV was outside the FDG-HV (group 1, Figure 6.3A). In 7 lesions, 2 to $12 \mathrm{~cm}^{3}$ of the HX4-HV was outside the FDG-HV (group 2, Figure 6.3B). Finally, in 2 patients a clearly distinct uptake pattern was observed between the two tracers and hypoxic volumes of 46 and $102 \mathrm{~cm}^{3}$ were observed outside the FDG high-uptake region, which were $73 \%$ and $78 \%$ of the total HX4-HF, respectively (group 3, Figure $6.3 \mathrm{C}$ ). The primary tumor volume was significantly correlated to the group the lesion was assigned to $(R=0.75 ; P<0.01)$.
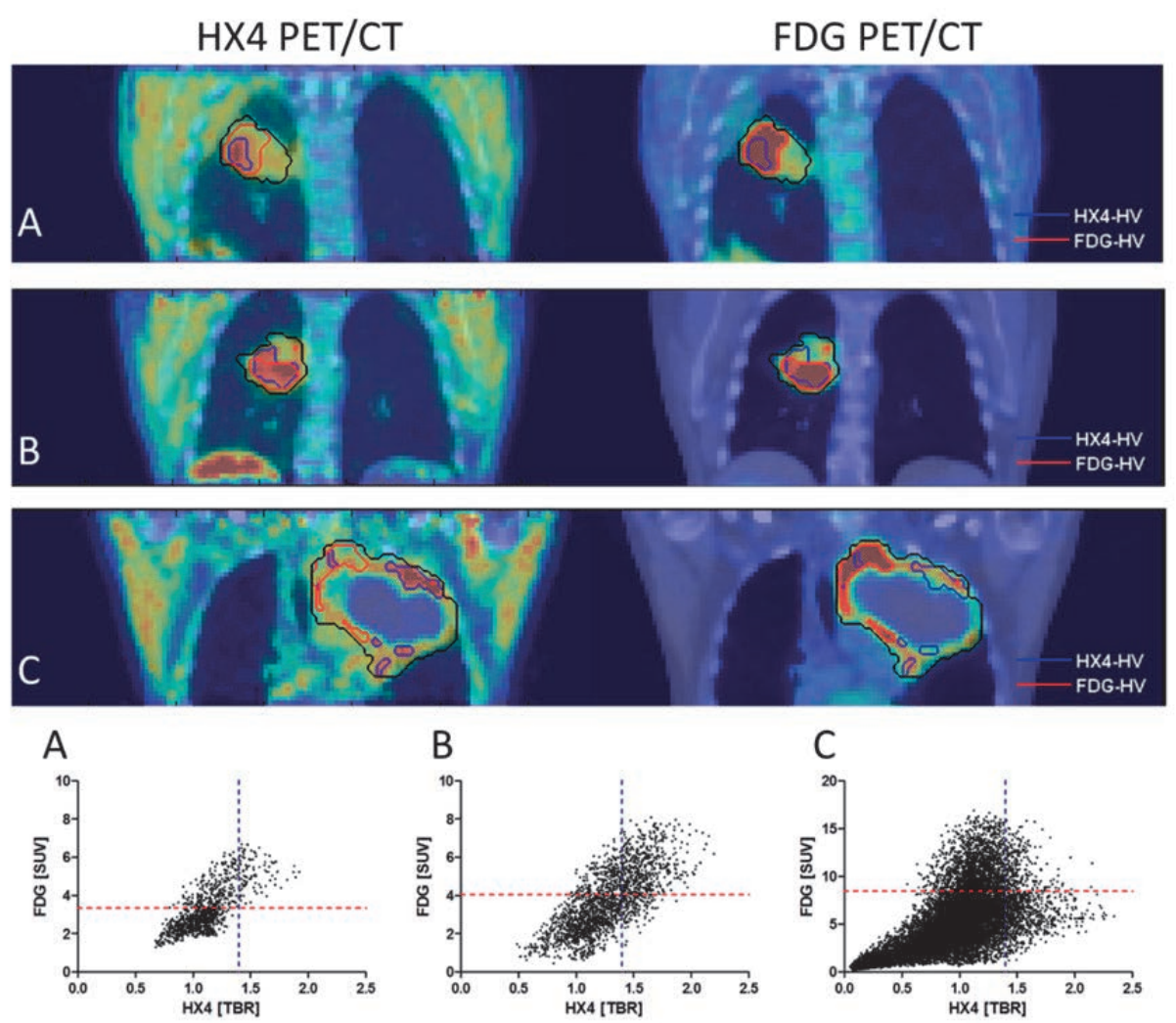

Figure 6.3: Visual and voxel-wise comparison of HX4- and FDG-PET/CT. A), HX4-HV within the FDG-HV. B), partial overlap between HX4-HV and FDG-HV. C), two distinct uptake patterns. 


\section{DISCUSSION}

This study was initiated to assess the correlation of (spatial) uptake patterns of hypoxia (using HX4-PET) and tumor metabolism (using FDG-PET) in primary NSCLC and associated lymph node metastases. Both biological features are known to have an adverse impact on treatment outcome in NSCLC. FDG-PET is routinely used in clinical practice for staging, radiotherapy planning and treatment response monitoring, while the use of hypoxia (HX4) PET imaging is still limited to clinical trials. We show in 25 NSCLC patients, with different histopathological subtypes, that HX4-PET imaging provides additional information to FDG PET which can be used to individualize patient treatment.

The relationship between HX4- and FDG-PET was investigated at a tumor level by comparing the overall uptake within the GTVs of the primary tumor and lymph node metastases. Significant correlations were observed between GTV, HX4- and FDG-PET image parameters. Previous studies comparing the overall uptake of hypoxia PET and FDG PET showed varying results. No correlations were observed by Bollineni and colleagues (7) or Cherk and colleagues (21), while Vera and colleagues (22) reported a significant correlation. Gagel and colleagues (23) compared FDG and FMISO uptake to the gold standard of hypoxia measurements ( $\mathrm{pO}_{2}$ polarography) and observed a moderate correlation for FMISO but no correlation for FDG. However, because both FDG-SUV max $_{\text {ax }}$ and GTV are predictors of survival in NSCLC (24-26) and the amount of tumor hypoxia is related to outcome after radiotherapy (27), the reported correlation between hypoxia and FDG PET is plausible.

Information about hypoxia on a tumor level can be used in clinical practice to select patients who may benefit from hypoxia modification before or during anti-cancer treatment. Previous studies have shown that the addition of hypoxic modification during radiotherapy results in an increased therapeutic benefit (28). Recently, Arrieta and colleagues (29) investigated in NSCLC patients the use of nitroglycerin (an organic nitrate which causes vasodilatation, increased blow flow and reduces the expression of HIF1- $\alpha$ ) in combination with chemo-radiation. In this study promising response rates were observed, however, there was also (mild) increased toxicity (e.g. headache, hypotension) due to nitroglycerin administration. Another promising compound is the hypoxia-activated prodrug TH-302 which releases bromo isophosphoramide mustard, a potent DNA alkylating agent, in hypoxic regions. Saggar and Tannock (30) recently demonstrated that TH-302 administered together with chemotherapy enhances the antitumor effect but also increases toxicity. From these recent studies, we acknowledge the therapeutic effect of additional anti-hypoxia treatment, but also the importance to limit unnecessary toxicity by selecting patients who will benefit from these modifications. We show that we can noninvasively visualize and quantify tumor hypoxia, using HX4 PET, in patients with NSLSC. In addition, our results show that patients with a larger tumor size and higher FDG uptake are more likely to have a larger hypoxic volume. This combination (GTV size and FDG uptake) could be used as a surrogate for hypoxia PET imaging, however, despite the correlation between hypoxia and FDG parameters, the distinction between hypoxic and 
non-hypoxic tumors based on FDG PET can be misleading, since non-hypoxic tumors are present in a broad range of FDG uptake (FDG-SUV max $_{1} 3.5$ - 17.5 also shown in Figure 6.1B).

It is important to note that a correlation at a global tumor level provides no information about the intratumoral heterogeneity. At the moment, limited data is available concerning the correlation of hypoxia PET and FDG PET at a subvolume (e.g. voxel) level in NSCLC (7, 31). The spatial concordance and discordance of both PET modalities is of interest for radiotherapy boosting strategies. FDG-PET is already used in the context of clinical trials to boost highly metabolic tumor subvolumes $(18,32)$. We hypothesize that hypoxia PET imaging may be more selective in defining radioresistant voxels within the GTV, and can provide complementary information regarding the definition of radiotherapy boost volumes. A voxel-wise comparison was performed to evaluate the spatial distribution of the HX4 and FDG uptake. A reasonable correlation between both tracers was observed in the majority of patients. This is in contradiction to the previous published results of Bollineni and colleagues (7), who observed no correlation between FDG and the hypoxia PET tracer FAZA. This disagreement can probably be explained by the definition of the target lesion. Bollineni and colleagues used an FDG based threshold to define the target lesion, thereby excluding voxels with a low FDG uptake. Conversely, Lohith and colleagues (31) reported a similar spatial distribution of $\left[{ }^{62} \mathrm{Cu}\right.$ ATSM and FDG in 5 patients with an adenocarcinoma of the lung, which was not present in patients with SCC. Also, they observed a difference in intra-tumoral distribution between adenocarcinoma and SCC, which was not observed in our cohort of patients with NSCLC. It is well described that hypoxia leads to an increased uptake of glucose through various molecular mechanisms (33). Nevertheless, an increased glycolysis is also observed without hypoxia, e.g. by c-myc aberrations (34). From a molecular point of view, it is therefore logical that FDG uptake and hypoxia is partially overlapping and is highly dependent on the genetics of the tumour.

Thresholds were defined arbitrarily to define regions with a high or low uptake on both FDG- and HX4-PET. The high FDG-PET volume was defined based on the ongoing NSCLC boost trial (18), whereas the high HX4 region was based on previous publications, indicating that a threshold of TBR $>1.4$ is rational to define hypoxia $(6,8,35,36)$. These thresholds showed the HX4-HV to be three times smaller on average than the FDG-HV.

This work can be used in clinical setting to divide patients with a hypoxic lesion into different groups, stratifying lesions with an agreement or disagreement between the HX4and FDG-PET uptake pattern. In the patients with a concordance, the use of HX4 PET has limited additional value for the selection of the radiotherapy boost volume, however, this volume could be limited to HX4-high areas only, facilitating further dose escalation without comprising the surrounding healthy tissue. In other patients a (partial) discordance between the HX4 and FDG PET uptake pattern was observed. In these patients, the boost region could be adjusted to either HX4 PET or a combination of HX4 and FDG PET with the aim to improve loco-regional control. On the basis of the current analysis, a radiation boost to the FDG high area (SUV > 50\% SUV $_{\max }$ ) would on average miss $24 \%$ of the hypoxic volume, which seems in agreement with the residual activity after radiotherapy outside the high-FDG area as reported by Aerts and colleagues (11). Previous studies have already shown that radiotherapy dose distribution based on tumor hypoxia is possible and promis- 
ing $(37,38)$. Currently there are strategies available to investigate the original location of local recurrences inside the tumor volume (39). These studies will characterize the subvolumes inside the heteregenous tumour that are difficult to control. Ultimately, the effect of tumour subvolume characterization and targeting, by radiotherapy or other therapeutic interventions, needs to be assessed in a randomized trial.

This study has several limitations. First, most patients received chemotherapy before the start of radiotherapy and PET imaging. Chemotherapy can reduce the amount of tumor hypoxia and downregulates metabolism, resulting in a decreased uptake of HX4 and FDG (40). However, the focus of our research is on the correlation between both imaging modalities, therefore treatment differences between patients are less relevant. In addition, it is most important to have recent PET information before the start of (adaptive) radiotherapy. Second, we were not able to validate the current imaging observations on tumor specimens. Nevertheless, van Baardwijk and colleagues (41) showed previously that FDG-PET imaging is correlated to GLUT-1 and HIF-1 $\alpha$ expression in NSCLC patients and Dubois and colleagues (8) showed a high correlation between HX4-PET uptake and pimonidazole staining in a rat rhabdomyosarcoma model. Third, the study acquired PET scans in free-breathing, which might cause blurring of the PET signal. Although, since both the FDG and HX4 scans were obtained in this setting, we do not expect any substantial bias for the comparison. Furthermore, advanced stage tumors are known to show little breathing induced motion $(42,43)$. Fourth, the FDG-PET/CT was rigidly registered to the $\mathrm{HX} 4-\mathrm{PET} / \mathrm{CT}$ scan to compare spatial uptake patterns. Small errors in registration can have a significant effect on correlation (44). However, patients in the current study were aligned in radiotherapy treatment position providing a strong basis for accurate registration. Fifth, there was a small time interval between the FDG- and HX4-PET/CT scan. Changes in anatomy, tumor metabolism or hypoxia may have occurred in this interval and influenced the comparison results. The time interval in our study was short (median, 3 days) and no interventions (e.g., chemotherapy or radiotherapy) were performed between the two scans, limiting the chances of anatomical or physiological changes. Finally, the usability of a tracer for radiation dose-painting is dependent on its spatial reproducibility. Aerts and colleagues (45) showed that the location of low and high FDG volumes were stable during radiotherapy. The short-time reproducibility for HX4 ( 2 vs. 4 hours) was confirmed, but the long-term reproducibility is still unknown (6). However, a high reproducibility has been reported by Busk and colleagues (46) and Okamoto and colleagues (47) for the alternative hypoxia tracers FMISO and FAZA.

In conclusion, there is a positive correlation between GTV, FDG- and HX4-uptake parameters on a tumor level. The hypoxic tumor volume is on average three times smaller than the metabolic active tumor volume. Approximately half of the lesions showed a good spatial correlation between the PET tracers. In the other cases, a (partial) mismatch was observed. Hypoxia PET imaging gives complimentary information to metabolic FDG imaging, which can potentially be used to individualize patient treatment by selecting patients for treatment with hypoxic sensitizers or hypoxia PET based radiotherapy dose escalation. 


\section{ACKNOWLEDGEMENTS}

The authors would like to thank the patients who agreed to participate, C. Overhof for handling the data-management for both clinical trials and R. Franssen for the data acquisition. This study was financially supported by the NIH(NIH-USA U01 CA 143062-01, Radiomics of NSCLC), the CTMM framework (AIRFORCE project, grant 030-103), the EU 6th and 7th framework program (METOXIA, EURECA, ARTFORCE), euroCAT (IVA Interreg www.eurocat.info), and the Kankeronderzoekfonds Limburg of the Health Foundation Limburg and the Dutch Cancer Society (KWF UM 2011-5020, KWF UM 2009-4454, KWF MAC 2011-4970, KWF MAC 2013-6425). 
128 Chapter 6

\section{SUPPLEMENTARY DATA}

Table S6.1: Patient characteristics.

N $\%$

\section{Gender}

Male

Female

\section{Trial}

PET-Boost

Stage

IB

IIB

2

8

IIIA

IIIB

IV

\section{Pathology}

Adenocarcinoma

$13 \quad 52$

Squamous cell carcinoma

Large cell carcinoma

\section{Treatment}

Radical radiotherapy

Sequential chemo-radiation

$3 \quad 8$

Concurrent chemo-radiation

Chemotherapy before imaging

$\begin{array}{lll}\text { None } & 3 & 12 \\ 1 \text { cycle } & 15 & 60 \\ 2 \text { cycles } & 4 & 16 \\ 3 \text { cycles } & 3 & 12\end{array}$
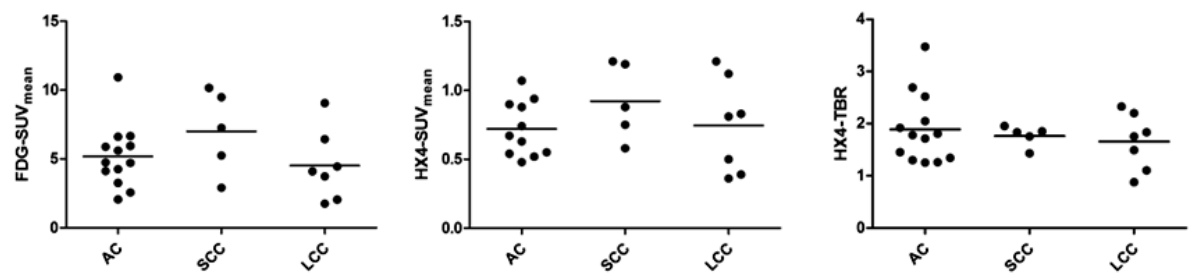

Figure S6.1: Example of the FDG and HX4 parameters (FDG-SUV mean, HX4-SUV mean and HX4-TBR) sub-classified by tumor pathology. Adenocarcinoma (AC), squamous cell carcinoma (SCC), large cell carcinoma (LCC). 
Table S6.2: Pearson's correlation coefficient $(R)$ and corresponding p-values of GTVIn based parameters on FDG and HX4 PET

\begin{tabular}{|c|c|c|c|c|c|c|}
\hline & Volume $\mathrm{C}$ & HX4 -SUV mean $_{\text {mat }}$ & HX4-SUV max $_{\text {max }}$ & HX4-TBR max $_{\text {max }}$ & HX4-HF & HX4-HV \\
\hline \multicolumn{7}{|c|}{ Volume GTVIn } \\
\hline$R$ & - & 0.19 & 0.54 & 0.72 & 0.43 & 0.95 \\
\hline$P$ & - & 0.48 & 0.03 & 0.001 & 0.07 & $<0.0001$ \\
\hline \multicolumn{7}{|c|}{ FDG-SUV ${ }_{\text {mean }}$} \\
\hline$R$ & 0.30 & 0.06 & 0.33 & 0.50 & 0.49 & 0.25 \\
\hline$p$ & 0.21 & 0.83 & 0.20 & 0.03 & 0.03 & 0.31 \\
\hline \multicolumn{7}{|c|}{ FDG-SUV $_{\max }$} \\
\hline$R$ & 0.18 & -0.08 & 0.18 & 0.37 & 0.29 & 0.09 \\
\hline$p$ & 0.45 & 0.77 & 0.49 & 0.12 & 0.23 & 0.71 \\
\hline \multicolumn{7}{|c|}{ FDG-HF } \\
\hline$R$ & 0.04 & 0.21 & 0.06 & -0.08 & 0.09 & 0.11 \\
\hline$P$ & 0.87 & 0.41 & 0.81 & 0.74 & 0.71 & 0.65 \\
\hline \multicolumn{7}{|c|}{ FDG-HV } \\
\hline$R$ & 0.97 & 0.24 & 0.54 & 0.66 & 0.45 & 0.96 \\
\hline$p$ & $<0.0001$ & 0.35 & 0.03 & $<0.01$ & 0.05 & $<0.0001$ \\
\hline
\end{tabular}

Table S6.3: Average distribution (mean+SD) of high (+) and low (-), HX4 and FDG uptake within the primary GTV.

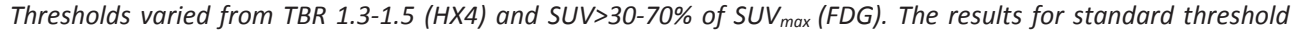
used in this article are presented bold and underlined; the two alternative thresholds visualized in this article are presented in bold.

\begin{tabular}{|c|c|c|c|c|c|}
\hline & \multicolumn{5}{|l|}{ FDG threshold } \\
\hline & $>30 \%$ SUVmax & $>40 \%$ SUVmax & $>50 \%$ SUVmax & $>60 \%$ SUVmax & $>70 \%$ SUVmax \\
\hline \multicolumn{6}{|c|}{ HX4 threshold TBR>1.3 } \\
\hline $\mathrm{FDG}+\mathrm{HX} 4+$ & $18.9 \pm 18.1$ & $17.5 \pm 17.1$ & $15.1 \pm 15.1$ & $11.8 \pm 12.2$ & $7.6 \pm 8.6$ \\
\hline $\mathrm{FDG}+\mathrm{HX} 4-$ & $54.4 \pm 22.3$ & $39.4 \pm 21.9$ & $26.3 \pm 19.3$ & $16.4 \pm 16.2$ & $8.7 \pm 11.4$ \\
\hline FDG-HX4+ & $1.3 \pm 4.5$ & $2.8 \pm 6.4$ & $5.1 \pm 8.1$ & $8.4 \pm 10.1$ & $12.6 \pm 13.0$ \\
\hline FDG-HX4- & $25.4 \pm 19.1$ & $40.4 \pm 22.1$ & $53.5 \pm 22.2$ & $63.4 \pm 21.5$ & $71.0 \pm 19.9$ \\
\hline \multicolumn{6}{|c|}{ HX4 threshold TBR>1.4 } \\
\hline $\mathrm{FDG}+\mathrm{HX} 4+$ & $12.7 \pm 14.5$ & $11.8 \pm 13.7$ & $\underline{10.4 \pm 12.2}$ & $8.4 \pm 10.0$ & $5.6 \pm 7.0$ \\
\hline $\mathrm{FDG}+\mathrm{HX} 4-$ & $60.6 \pm 21.1$ & $45.1 \pm 21.5$ & $\underline{31.1 \pm 19.5}$ & $19.8 \pm 16.4$ & $10.7 \pm 11.7$ \\
\hline FDG-HX4+ & $0.9 \pm 3.7$ & $1.8 \pm 6.3$ & $\underline{3.2 \pm 6.5}$ & $5.2 \pm 8.1$ & $8.0 \pm 10.4$ \\
\hline FDG-HX4- & $25.8 \pm 19.2$ & $41.3 \pm 22.0$ & $55.3 \pm 21.9$ & $66.6 \pm 20.4$ & $75.7 \pm 17.9$ \\
\hline \multicolumn{6}{|c|}{ HX4 threshold TBR>1.5 } \\
\hline FDG+HX4+ & $8.2 \pm 11.5$ & $7.7 \pm 11.0$ & $6.8 \pm 9.9$ & $5.6 \pm 8.1$ & $3.8 \pm 5.5$ \\
\hline $\mathrm{FDG}+\mathrm{HX} 4-$ & $65.1 \pm 20.5$ & $49.2 \pm 21.4$ & $34.6 \pm 19.7$ & $22.6 \pm 16.6$ & $12.6 \pm 12.0$ \\
\hline FDG-HX4+ & $0.7 \pm 3.0$ & $1.2 \pm 4.2$ & $2.1 \pm 5.2$ & $3.3 \pm 6.4$ & $5.1 \pm 8.4$ \\
\hline FDG-HX4- & $26.0 \pm 19.3$ & $41.9 \pm 21.9$ & $56.5 \pm 21.4$ & $68.5 \pm 19.4$ & $78.5 \pm 16.4$ \\
\hline
\end{tabular}




\section{REFERENCES}

1. Siegel R, Naishadham D, Jemal A. Cancer statistics, 2013. CA Cancer J Clin. 2013;63:11-30.

2. Auperin A, Le Pechoux C, Rolland E, Curran WJ, Furuse K, Fournel P, et al. Metaanalysis of concomitant versus sequential radiochemotherapy in locally advanced non-small-cell lung cancer. J Clin Oncol. 2010;28:2181-90.

3. Nordsmark M, Bentzen SM, Rudat V, Brizel D, Lartigau E, Stadler P, et al. Prognostic value of tumor oxygenation in 397 head and neck tumors after primary radiation therapy. An international multi-center study. Radiother Oncol. 2005;77:18-24.

4. Zips D, Zophel K, Abolmaali N, Perrin R, Abramyuk A, Haase R, et al. Exploratory prospective trial of hypoxia-specific $\mathrm{PET}$ imaging during radiochemotherapy in patients with locally advanced head-and-neck cancer. Radiother Oncol. 2012;105:21-8.

5. Milosevic M, Warde $P$, Menard C, Chung $P$, Toi A, Ishkanian A, et al. Tumor hypoxia predicts biochemical failure following radiotherapy for clinically localized prostate cancer. Clin Cancer Res. 2012;18:2108-14.

6. Zegers $\mathrm{CM}$, van Elmpt W, Wierts R, Reymen B, Sharifi H, Ollers MC, et al. Hypoxia imaging with [(18)F]HX4 PET in NSCLC patients: Defining optimal imaging parameters. Radiother Oncol. 2013;109:58-64.

7. Bollineni VR, Kerner GS, Pruim J, Steenbakkers RJ, Wiegman EM, Koole MJ, et al. PET Imaging of Tumor Hypoxia Using 18F-Fluoroazomycin Arabinoside in Stage III-IV NonSmall Cell Lung Cancer Patients. J Nucl Med. 2013.

8. Dubois LJ, Lieuwes NG, Janssen MH, Peeters WJ, Windhorst AD, Walsh JC, et al. Preclinical evaluation and validation of [18F]HX4, a promising hypoxia marker for PET imaging. Proc Natl Acad Sci U S A. 2011;108:14620-5.

9. van Loon J, Janssen $M H$, Ollers $M$, Aerts HJ, Dubois L, Hochstenbag $M$, et al. PET imaging of hypoxia using [18F]HX4: a phase I trial. Eur J Nucl Med Mol Imaging. 2010;37:1663-8.

10. Chen L, Zhang Z, Kolb HC, Walsh JC, Zhang J, Guan Y. (1)(8)F-HX4 hypoxia imaging with PET/CT in head and neck cancer: a comparison with (1)(8)F-FMISO. Nucl Med Commun. 2012;33:1096-102.

11. Aerts HJ, van Baardwijk AA, Petit SF, Offermann C, Loon J, Houben R, et al. Identification of residual metabolic-active areas within individual NSCLC tumours using a pre-radiotherapy (18)Fluorodeoxyglucose-PET-CT scan. Radiother Oncol. 2009;91:386-92.

12. Abramyuk A, Tokalov S, Zophel K, Koch A, Szluha Lazanyi K, Gillham C, et al. Is pretherapeutical FDG-PET/CT capable to detect high risk tumor subvolumes responsible for local failure in non-small cell lung cancer? Radiother Oncol. 2009;91:399-404.

13. Spijkerman J, Fontanarosa D, Das M, van Elmpt W. Validation of nonrigid registration in pretreatment and follow-up PET/CT scans for quantification of tumor residue in lung cancer patients; 2014.

14. Lambin P, van Stiphout RG, Starmans MH, Rios-Velazquez E, Nalbantov G, Aerts HJ, et al. Predicting outcomes in radiation oncology--multifactorial decision support systems. Nat Rev Clin Oncol. 2013;10:27-40. 
15. Aerts HJ, Lambin P, Ruysscher DD. FDG for dose painting: a rational choice. Radiother Oncol. 2010;97:163-4.

16. Lambin P, Petit SF, Aerts HJ, van Elmpt WJ, Oberije CJ, Starmans MH, et al. The ESTRO Breur Lecture 2009. From population to voxel-based radiotherapy: exploiting intratumour and intra-organ heterogeneity for advanced treatment of non-small cell lung cancer. Radiother Oncol. 2010;96:145-52.

17. Pereira KM, Chaves FN, Viana TS, Carvalho FS, Costa FW, Alves AP, et al. Oxygen metabolism in oral cancer: HIF and GLUTs (Review). Oncol Lett. 2013;6:311-6.

18. van Elmpt W, De Ruysscher D, van der Salm A, Lakeman A, van der Stoep J, Emans D, et al. The PET-boost randomised phase II dose-escalation trial in non-small cell lung cancer. Radiother Oncol. 2012;104:67-71.

19. Doss M, Zhang JJ, Belanger MJ, Stubbs JB, Hostetler ED, Alpaugh K, et al. Biodistribution and radiation dosimetry of the hypoxia marker $18 \mathrm{~F}-\mathrm{HX} 4$ in monkeys and humans determined by using whole-body PET/CT. Nucl Med Commun. 2010;31:1016-24.

20. Boellaard R, Oyen WJ, Hoekstra CJ, Hoekstra OS, Visser EP, Willemsen AT, et al. The Netherlands protocol for standardisation and quantification of FDG whole body PET studies in multi-centre trials. European journal of nuclear medicine and molecular imaging. 2008;35:2320-33.

21. Cherk MH, Foo SS, Poon AM, Knight SR, Murone C, Papenfuss AT, et al. Lack of correlation of hypoxic cell fraction and angiogenesis with glucose metabolic rate in non-small cell lung cancer assessed by 18 F-Fluoromisonidazole and 18F-FDG PET. J Nucl Med. 2006;47:1921-6.

22. Vera P, Bohn P, Edet-Sanson A, Salles A, Hapdey S, Gardin I, et al. Simultaneous positron emission tomography (PET) assessment of metabolism with (1)(8)F-fluoro-2deoxy-d-glucose (FDG), proliferation with (1)(8)F-fluoro-thymidine (FLT), and hypoxia with (1)(8)fluoro-misonidazole (F-miso) before and during radiotherapy in patients with non-small-cell lung cancer (NSCLC): a pilot study. Radiother Oncol. 2011;98:109-16.

23. Gagel B, Piroth M, Pinkawa M, Reinartz P, Zimny M, Kaiser HJ, et al. pO polarography, contrast enhanced color duplex sonography (CDS), [18F] fluoromisonidazole and [18F] fluorodeoxyglucose positron emission tomography: validated methods for the evaluation of therapy-relevant tumor oxygenation or only bricks in the puzzle of tumor hypoxia? BMC Cancer. 2007;7:113.

24. Cistaro A, Quartuccio N, Mojtahedi A, Fania P, Filosso PL, Campenni A, et al. Prediction of 2 years-survival in patients with stage I and II non-small cell lung cancer utilizing (18)F-FDG PET/CT SUV quantification. Radiol Oncol. 2013;47:219-23.

25. Tanaka H, Hayashi S, Hoshi H. Pretreatment maximum standardized uptake value on 18F-fluorodeoxyglucose positron emission tomography is a predictor of outcome for stage I non-small cell lung cancer after stereotactic body radiotherapy. Asia Pac J Clin Oncol. 2013.

26. Satoh $Y$, Onishi H, Nambu A, Araki T. Volume-based parameters measured by using FDG PET/CT in patients with stage I NSCLC treated with stereotactic body radiation therapy: prognostic value. Radiology. 2014;270:275-81.

27. Eschmann SM, Paulsen F, Reimold M, Dittmann H, Welz S, Reischl G, et al. Prognostic impact of hypoxia imaging with 18F-misonidazole PET in non-small cell lung cancer and head and neck cancer before radiotherapy. J Nucl Med. 2005;46:253-60. 
28. Overgaard J. Hypoxic modification of radiotherapy in squamous cell carcinoma of the head and neck--a systematic review and meta-analysis. Radiother Oncol. 2011;100:22-32.

29. Arrieta O, Blake M, de la Mata-Moya MD, Corona F, Turcott J, Orta D, et al. Phase II study. Concurrent chemotherapy and radiotherapy with nitroglycerin in locally advanced non-small cell lung cancer. Radiother Oncol. 2014;111:311-5.

30. Saggar JK, Tannock IF. Activity of the hypoxia-activated pro-drug TH-302 in hypoxic and perivascular regions of solid tumors and its potential to enhance therapeutic effects of chemotherapy. Int J Cancer. 2014;134:2726-34.

31. Lohith TG, Kudo T, Demura Y, Umeda Y, Kiyono Y, Fujibayashi Y, et al. Pathophysiologic correlation between 62Cu-ATSM and 18F-FDG in lung cancer. J Nucl Med. 2009;50:1948-53.

32. Moller DS, Khalil AA, Knap MM, Muren LP, Hoffmann L. A planning study of radiotherapy dose escalation of PET-active tumour volumes in non-small cell lung cancer patients. Acta Oncol. 2011;50:883-8.

33. Vander Heiden MG, Cantley LC, Thompson CB. Understanding the Warburg effect: the metabolic requirements of cell proliferation. Science. 2009;324:1029-33.

34. Dang CV, Le A, Gao P. MYC-induced cancer cell energy metabolism and therapeutic opportunities. Clin Cancer Res. 2009;15:6479-83.

35. Dubois L, Landuyt W, Haustermans K, Dupont P, Bormans G, Vermaelen P, et al. Evaluation of hypoxia in an experimental rat tumour model by [(18)F]fluoromisonidazole PET and immunohistochemistry. Br J Cancer. 2004;91:1947-54.

36. Rasey JS, Koh WJ, Evans ML, Peterson LM, Lewellen TK, Graham MM, et al. Quantifying regional hypoxia in human tumors with positron emission tomography of [18F]fluoromisonidazole: a pretherapy study of 37 patients. Int J Radiat Oncol Biol Phys. 1996;36:417-28.

37. Petit SF, Dekker AL, Seigneuric R, Murrer L, van Riel NA, Nordsmark M, et al. Intravoxel heterogeneity influences the dose prescription for dose-painting with radiotherapy: a modelling study. Phys Med Biol. 2009;54:2179-96.

38. Thorwarth D, Eschmann SM, Paulsen F, Alber M. Hypoxia dose painting by numbers: a planning study. Int J Radiat Oncol Biol Phys. 2007;68:291-300.

39. Due AK, Vogelius IR, Aznar MC, Bentzen SM, Berthelsen AK, Korreman SS, et al. Recurrences after intensity modulated radiotherapy for head and neck squamous cell carcinoma more likely to originate from regions with high baseline [18F]-FDG uptake. Radiother Oncol. 2014;111:360-5.

40. Bittner MI, Wiedenmann N, Bucher S, Hentschel M, Mix M, Weber WA, et al. Exploratory geographical analysis of hypoxic subvolumes using 18F-MISO-PET imaging in patients with head and neck cancer in the course of primary chemoradiotherapy. Radiother Oncol. 2013;108:511-6.

41. van Baardwijk A, Dooms C, van Suylen RJ, Verbeken E, Hochstenbag M, DehingOberije $C$, et al. The maximum uptake of (18)F-deoxyglucose on positron emission tomography scan correlates with survival, hypoxia inducible factor-1alpha and GLUT1 in non-small cell lung cancer. Eur J Cancer. 2007;43:1392-8.

42. Bosmans G, van Baardwijk A, Dekker A, Ollers M, Boersma L, Minken A, et al. Intrapatient variability of tumor volume and tumor motion during conventionally 
fractionated radiotherapy for locally advanced non-small-cell lung cancer: a prospective clinical study. Int J Radiat Oncol Biol Phys. 2006;66:748-53.

43. $\mathrm{Yu} \mathrm{ZH}$, Lin SH, Balter $\mathrm{P}$, Zhang L, Dong L. A comparison of tumor motion characteristics between early stage and locally advanced stage lung cancers. Radiother Oncol. 2012;104:33-8.

44. Nyflot MJ, Harari PM, Yip S, Perlman SB, Jeraj R. Correlation of PET images of metabolism, proliferation and hypoxia to characterize tumor phenotype in patients with cancer of the oropharynx. Radiother Oncol. 2012;105:36-40.

45. Aerts HJ, Bosmans G, van Baardwijk AA, Dekker AL, Oellers MC, Lambin P, et al. Stability of 18F-deoxyglucose uptake locations within tumor during radiotherapy for NSCLC: a prospective study. Int J Radiat Oncol Biol Phys. 2008;71:1402-7.

46. Busk M, Mortensen LS, Nordsmark M, Overgaard J, Jakobsen S, Hansen KV, et al. PET hypoxia imaging with FAZA: reproducibility at baseline and during fractionated radiotherapy in tumour-bearing mice. European journal of nuclear medicine and molecular imaging. 2013;40:186-97.

47. Okamoto S, Shiga T, Yasuda K, Ito YM, Magota K, Kasai K, et al. High reproducibility of tumor hypoxia evaluated by $18 \mathrm{~F}$-fluoromisonidazole PET for head and neck cancer. J Nucl Med. 2013;54:201-7. 



\section{CHAPTER}

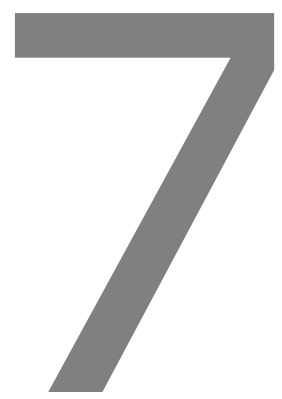

Imaging of tumour hypoxia and metabolism in patients with head and neck squamous cell carcinoma

Catharina M.L. Zegers, Wouter van Elmpt, Frank J.P. Hoebers, Esther G.C. Troost, Michel C. Öllers, Felix M. Mottaghy, Philippe Lambin

Published in: Acta Oncol. 2015 54(9): 1378-84 


\section{ABSTRACT}

Introduction: Tumour hypoxia and a high tumour metabolism increase radioresistance in patients with head and neck squamous cell carcinoma (HNSCC). The aim of this study was to evaluate the correlation between hypoxia $\left(\left[{ }^{18} \mathrm{~F}\right] \mathrm{HX} 4-\mathrm{PET}\right)$ and glucose metabolism $\left(\left[{ }^{18} \mathrm{~F}\right] \mathrm{FDG} \mathrm{PET}\right)$ molecular imaging.

Materials and methods: $\left[{ }^{18} \mathrm{~F}\right] \mathrm{HX} 4$ and $\left[{ }^{18} \mathrm{~F}\right] \mathrm{FDG}-\mathrm{PET} / \mathrm{CT}$ images of $20 \mathrm{HNSCC}$ patients were acquired prior to (chemo)radiotherapy, in an immobilization mask, with a median time interval of seven days (NCT01347281). Gross tumour volumes of the primary lesions $\left(G_{\text {prim }}\right)$ and pathological lymph nodes $\left(G V_{\text {In }}\right)$ were included in the analysis. $\left[{ }^{18} \mathrm{~F}\right] \mathrm{FDG}$ $\mathrm{PET} / \mathrm{CT}$ images were rigidly registered to the $\left[{ }^{18} \mathrm{~F}\right] \mathrm{HX} 4 \mathrm{PET} / \mathrm{CT}$ images. The maximum and mean standardized uptake values $\left(S_{U} U V_{\text {max }}, S U V_{\text {mean }}\right)$ within both GTVs were determined. In addition, the overlap was compared between the $\left[{ }^{18} \mathrm{~F}\right] \mathrm{HX} 4$ high volume $\left(\left[{ }^{18} \mathrm{~F}\right] \mathrm{HX} 4-\mathrm{HV}\right)$ with a tumour-to-muscle ratio $>1.4$ and the $\left[{ }^{18}\right.$ F FDG high volume $\left(\left[{ }^{18} \mathrm{~F}\right] \mathrm{FDG}-\mathrm{HV}\right)$ with an SUV $>$ $50 \%$ of the SUV $\max$. We report the mean \pm standard deviation.

Results: PET/CT scans including $20 \mathrm{GTV}_{\text {prim }}$ and $12 \mathrm{GTV}_{\text {In }}$ were analysed. There was a significant correlation between several $\left[{ }^{18} \mathrm{~F}\right] \mathrm{FDG}$ and $\left[{ }^{18} \mathrm{~F}\right] \mathrm{HX} 4$ parameters, the most pronounced being the correlation between $\left[{ }^{18} \mathrm{~F}\right] \mathrm{FDG}-\mathrm{HV}$ and $\left[{ }^{18} \mathrm{~F}\right] \mathrm{HX} 4-\mathrm{HV}(\mathrm{R}=0.93, \mathrm{p}<0.001)$. The fraction of the $\mathrm{GTV}_{\text {prim }}$ with a high $\mathrm{HX} 4$ uptake $(9 \pm 10 \%)$ was on average smaller than the FDG high fraction $(51 \pm 26 \%$; $P<0.001)$. In $65 \%(13 / 20)$ of the patients, the $\mathrm{GTV}_{\text {prim }}$ was hypoxic. In four of these patients the $\left[{ }^{18} \mathrm{~F}\right] \mathrm{HX} 4-\mathrm{HV}$ was located within the $\left[{ }^{18} \mathrm{~F}\right] \mathrm{FDG}-\mathrm{HV}$, whereas for the remaining nine $\mathrm{GTV}_{\text {prim }}$ a partial mismatch was observed. In these nine tumours $25 \pm 21 \%$ (range: $5-64 \%$ ) of the HX4-HV was located outside the FDG-HV.

Conclusions: There is a correlation between $\left[{ }^{18} \mathrm{~F}\right] \mathrm{HX} 4$ and $\left[{ }^{18} \mathrm{~F}\right] \mathrm{FDG}$ uptake parameters on a global tumour level. In the majority of lesions a partial mismatch between the $\left[{ }^{18} \mathrm{~F}\right] \mathrm{HX} 4$ and $\left[{ }^{18} \mathrm{~F}\right]$ FDG high uptake volumes was observed, therefore $\left[{ }^{18} \mathrm{~F}\right] \mathrm{FDG}$ PET imaging cannot be used as a surrogate for hypoxia. $\left[{ }^{18} \mathrm{~F}\right] \mathrm{HX} 4 \mathrm{PET}$ provides complementary information to $\left[{ }^{18}\right.$ F]FDG PET imaging. 


\section{INTRODUCTION}

Tumour cell hypoxia decreases the effectiveness of anti-cancer treatment (i.e., surgery, radiotherapy and systemic treatment) and increases tumour aggressiveness in a number of solid tumours (1). Tumour cell hypoxia is present in the majority of head and neck squamous cell carcinomas (HNSCC) and can be visualized and quantified using positron emission tomography (PET) imaging $(2,3)$. This non-invasive imaging technique provides the opportunity to perform repeated tumour hypoxia measurements of the entire tumour, and gives important information to predict locoregional control and survival $(4,5)$.

3- $\left[{ }^{18} \mathrm{~F}\right]$ fluoro-2-(4-((2-nitro-1H-imidazol-1-yl)methyl)-1H-1,2,3-triazol-1-yl)propan-1-ol $\left(\left[{ }^{18} \mathrm{~F}\right] \mathrm{HX} 4\right)$ is a hypoxia PET tracer used to visualise and quantify tumour hypoxia. In previous pre-clinical studies, $\left[{ }^{18} \mathrm{~F}\right] \mathrm{HX} 4$ was validated as a hypoxia tracer and the repeatability of the tracer uptake was assessed $(6,7)$. In addition, in patients with non-small cell lung cancer (NSCLC), $\left[{ }^{18} \mathrm{~F}\right] \mathrm{HX} 4$ was found to provide additional information with respect to the metabolic PET tracer $\left[{ }^{18} \mathrm{~F}\right] \mathrm{FDG}(8)$.

$\left[{ }^{18}\right.$ F]FDG PET imaging is the most frequently used molecular imaging modality in clinical practice to detect and stage malignancies. Sub-volumes of the tumour displaying a high glucose metabolism as identified by $\left[{ }^{18} \mathrm{~F}\right]$ FDG PET were shown to often be the source of a local recurrences after (chemo)radiotherapy in $\operatorname{NSCLC}$ and $\operatorname{HNSCC}(9,10)$.

The uptake of $\left[{ }^{18} \mathrm{~F}\right] \mathrm{FDG}$ is dependent on the rate of glycolysis and the upregulation of glucose transporters (11). In the absence of oxygen, cells undergo several biological responses. Hypoxic tumors require an increased glycolysis to survive. In addition the hypoxia-inducible-factor $1 \alpha$ pathway is activated, which can causes an upregulation of the glucose transporters (1). This might indicate a relationship between hypoxia and metabolism. Nevertheless, most cancer cells produce energy by a high rate of aerobic glycolysis, independent of the presence of oxygen, the 'Warburg effect' (12). Multiple cellular pathways can lead to the glycolytic phenotype, therefore an altered glucose metabolism can also be observed without hypoxia(11)

The aim of this study was to characterize the relationship between the PET tracers $\left[{ }^{18} \mathrm{~F}\right]$ FDG (glucose metabolism) and $\left[{ }^{18} \mathrm{~F}\right] \mathrm{HX} 4$ (hypoxia) in the primary tumour and metastatic lymph nodes of patients with HNSCC. We evaluate the tracer uptake on a global tumour level and assess the spatial overlap between the high uptake volumes of both PET tracers. 


\section{MATERIALS AND METHODS}

\section{Patients}

$\left[{ }^{18}\right.$ F]FDG and $\left[{ }^{18} \mathrm{~F}\right] \mathrm{HX} 4 \mathrm{PET} / \mathrm{CT}$ images of 20 patients (17 male, 3 female) with a locally advanced HNSCC were acquired before the start of external beam (chemo)radiotherapy. The average age of the patients was 60 years (range $45-77$ years). Tumour stages were; cT1-4, cN0-2b and cM0, with a stage grouping from II-IVA, located in the larynx $(\mathrm{N}=8)$, oropharynx $(N=7)$ and hypopharynx $(N=5)$. The study was approved by the Ethical Review Committee of Maastricht University Medical Centre and registered on clinicaltrial.gov (NCT01347281). All patients provided written informed consent before study entry.

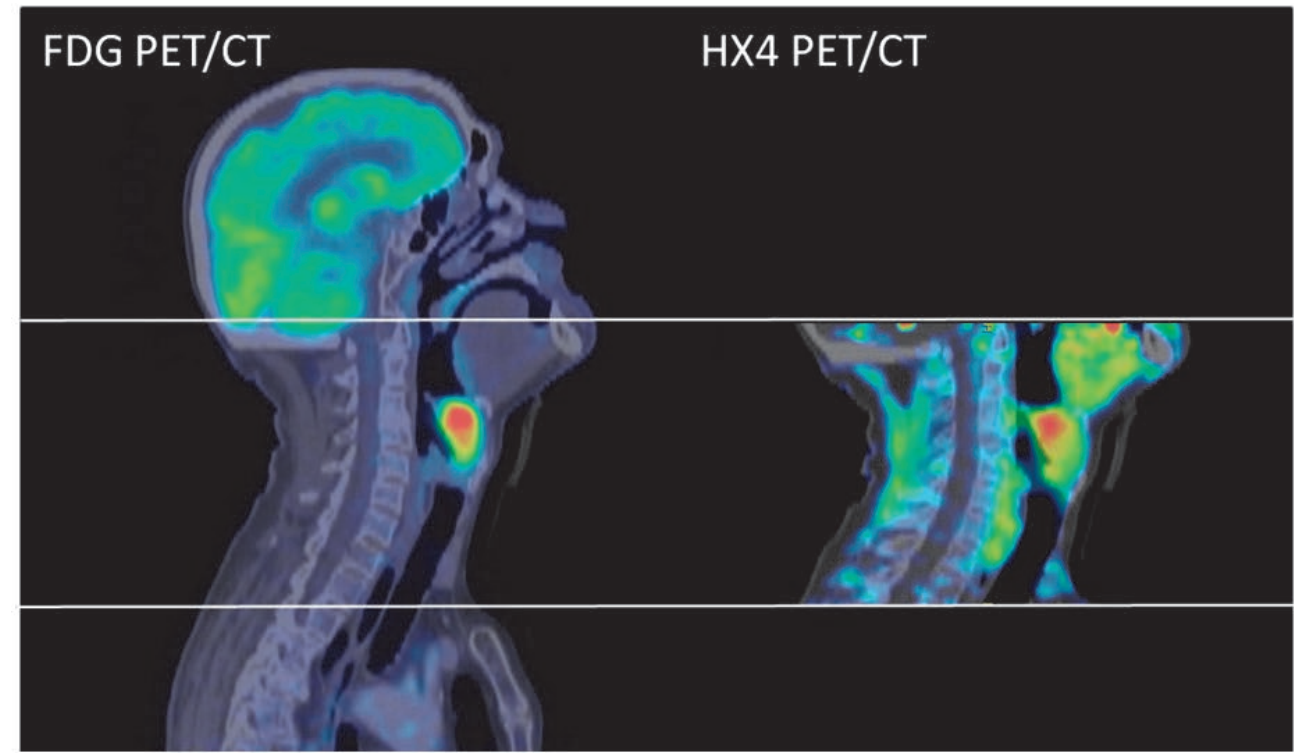

Figure 7.1: Good spatial overlap between $\left[{ }^{18} \mathrm{~F}\right] \mathrm{FDG}$ and $\left[{ }^{18} \mathrm{~F}\right] \mathrm{HX} 4$ uptake in a patient with a cT3NOMO laryngeal carcinoma prior to start of radiotherapy. The interval between both scans was 8 days.

\section{PET/CT imaging}

$\left[{ }^{18} \mathrm{~F}\right] \mathrm{HX} 4$ was produced as described previously $(6,13)$. After intravenous administration of an average $( \pm S D)$ dose of $378 \pm 84 \mathrm{MBq}\left[{ }^{18} \mathrm{~F}\right] \mathrm{HX} 4, \mathrm{PET} / \mathrm{CT}$ imaging was performed at four hours post-injection (p.i.) for 20 minutes in a single bed position. The injected activity of $\left[{ }^{18} \mathrm{~F}\right]$ FDG was based on the patient's bodyweight according to the national guidelines (14). Patients fasted for at least six hours before the intravenous administration of $\left[{ }^{18} \mathrm{~F}\right] \mathrm{FDG}$ and blood glucose levels were measured. Imaging was performed one hour after the injection of $\left[{ }^{18} \mathrm{~F}\right]$ FDG with a scan duration of five minutes per bed position. .

$\left[{ }^{18} \mathrm{~F}\right] \mathrm{HX} 4$ and $\left[{ }^{18} \mathrm{~F}\right] \mathrm{FDG} \mathrm{PET} / \mathrm{CT}$ scans were performed in radiotherapy position, with the patient positioned on a flat table top using an immobilization mask and a movable laser alignment system. Image acquisitions were performed on the same PET/CT scanner (Biograph 40, Siemens Healthcare, Erlangen, Germany); scatter and attenuation correction 
were applied; and PET images were reconstructed using OSEM 2D (Ordered Subset Expectation Maximization, four iterations, eight subsets) and a Gaussian filter of $5 \mathrm{~mm}$. The median interval between both PET scans was seven days (range: 4-28 days).

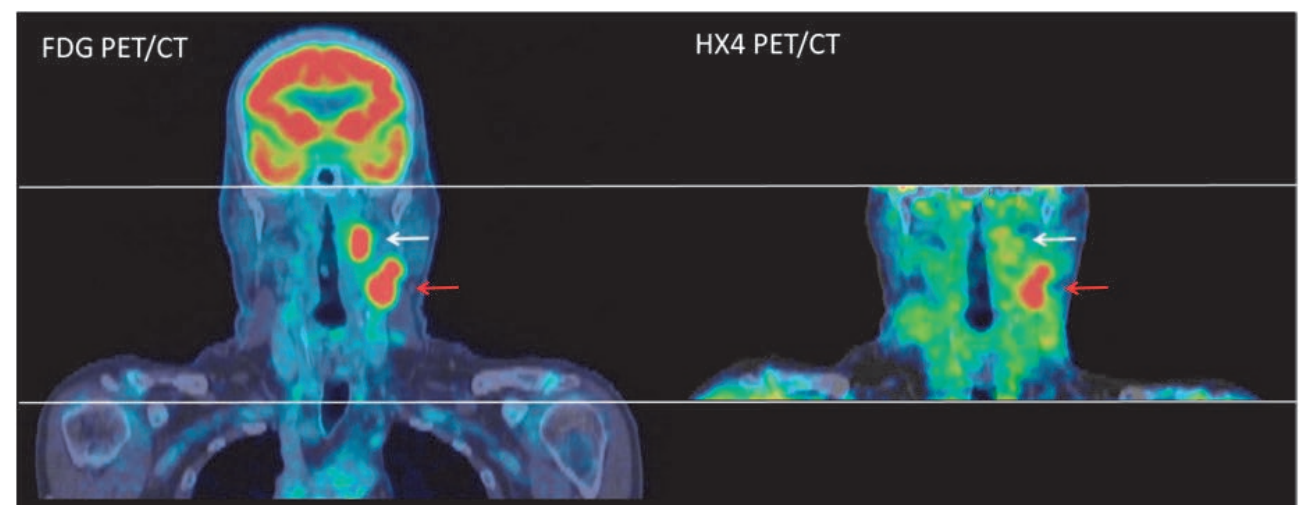

Figure 7.2: $\left[{ }^{18}\right.$ F]FDG and $\left[{ }^{18}\right.$ F]HX4 uptake in a patient with a cT2N2bMO squamous cell carcinoma of the oropharynx. Even though both metastatic lymph nodes are highly $\left[{ }^{18}\right.$ F]FDG avid, only the lower lymph node (red arrow) also shows high $\left[{ }^{18}\right.$ F]HX4 PET uptake, whereas the other lymph node does not (white arrow). The Interval between both scans was 5 days.

\section{Image analysis}

The gross tumour volume of the primary tumour $\left(\mathrm{GTV}_{\text {prim }}\right)$ and involved lymph nodes $\left(G V_{\text {In }}\right)$, were delineated on the $\left[{ }^{18}\right.$ F]FDG PET/CT, by two experienced radiation oncologists in consensus. The $\left[{ }^{18} \mathrm{~F}\right] \mathrm{FDG} \mathrm{CT}$ was rigidly registered to the $\left[{ }^{18} \mathrm{~F}\right] \mathrm{HX} 4 \mathrm{CT}$. The same transformation was subsequently applied to the $\left[{ }^{18} \mathrm{~F}\right] \mathrm{FDG}$ PET scan and the GTVs to co-register all images and contours.

The maximum and mean standardized uptake values $\left(S_{U} V_{\max }\right.$ and $\left.\mathrm{SUV}_{\text {mean }}\right)$, were determined within the $\mathrm{GTV}_{\text {prim }}$ and $\mathrm{GTV}_{\text {In }}$ on the $\left[{ }^{18} \mathrm{~F}\right] \mathrm{FDG}$ and $\left[{ }^{18} \mathrm{~F}\right] \mathrm{HX} 4$ PET scans. For the $\left[{ }^{18} \mathrm{~F}\right] \mathrm{HX} 4$ PET scans, the maximum tumour-to-muscle ratio $\left(\mathrm{TMR}_{\max }\right)$ was additionally calculated, being the SUV $V_{\text {max }}$ in the tumour divided by the SUV $V_{\text {mean }}$ in the trapezius muscles. The volume of interest in the trapezius muscles (left and right) were delineated on multiple slices of the CT scan. Subsequently, the $\left[{ }^{18} \mathrm{~F}\right] \mathrm{HX} 4$ high-fraction ( $\left.\left[{ }^{18} \mathrm{~F}\right] \mathrm{HX} 4-\mathrm{HF}\right)$ and $\left[{ }^{18} \mathrm{~F}\right] \mathrm{HX} 4$ high-volume $\left(\left[{ }^{18} \mathrm{~F}\right] \mathrm{HX} 4-\mathrm{HV}\right)$ were defined as the fraction/volume of the GTV with a TMR $>1.4$. The $\left[{ }^{18} \mathrm{~F}\right]$ FDG high-fraction $\left(\left[{ }^{18} \mathrm{~F}\right] \mathrm{FDG}-\mathrm{HF}\right)$ and $\left[{ }^{18} \mathrm{~F}\right] \mathrm{FDG}$ high-volume $\left(\left[{ }^{18} \mathrm{~F}\right] \mathrm{FDG}\right.$ HV) were calculated using the fraction/volume of the GTV with an SUV above $50 \%$ of the SUV $_{\text {max }}$, based on the ongoing clinical trials applying an $\left[{ }^{18}\right.$ F]FDG PET radiation dose redistribution $(15,16)$.

In addition, a voxel-wise comparison of the $\left[{ }^{18} \mathrm{~F}\right] \mathrm{FDG}$ and $\left[{ }^{18} \mathrm{~F}\right] \mathrm{HX} 4$ uptake within the GTV $_{\text {prim }}$ and GTV In was performed and DICE similarity coefficients were calculated using:

$$
D I C E=2 \frac{\mathrm{HX}_{\mathrm{HF}} \cap \mathrm{FDG}_{\mathrm{HF}}}{\mathrm{HX}_{\mathrm{HF}}+\mathrm{FDG}_{\mathrm{HF}}}
$$




\section{Statistical analysis}

For all parameters mean \pm 1 standard deviation (SD) are reported. To evaluate correlations between the tumour volume and $\left[{ }^{18} \mathrm{~F}\right] \mathrm{HX} 4$ and $\left[{ }^{18} \mathrm{~F}\right] \mathrm{FDG}$ imaging parameters, and to quantify the voxel-wise comparison of the $\left[{ }^{18} \mathrm{~F}\right] \mathrm{HX} 4$ and $\left[{ }^{18} \mathrm{~F}\right] \mathrm{FDG}$ uptake, linear regressions were performed and Pearson correlation coefficients were calculated. A Wilcoxon signed rank test was performed to evaluate a significant difference between the high uptake volumes. A p-value $<0.05$ was assumed to be statistically significant.

Table 7.1: $\left[{ }^{18} F\right] F D G$ - and $\left[{ }^{18} F\right] H X 4$-uptake parameters (mean $\pm S D$ ) for the primary lesions (GTVprim) and involved lymph nodes (GTVIn).

\begin{tabular}{|c|c|c|}
\hline & $\begin{array}{l}\text { GTVprim } \\
\text { average (range) }\end{array}$ & $\begin{array}{l}\text { GTVIn } \\
\text { average (range) }\end{array}$ \\
\hline$N$ & 20 & 12 \\
\hline Volume GTV & $\begin{array}{l}16 \pm 12 \mathrm{~cm}^{3} \\
\left(2-46 \mathrm{~cm}^{3}\right)\end{array}$ & $\begin{array}{l}21 \pm 30 \mathrm{~cm}^{3} \\
\left(1-105 \mathrm{~cm}^{3}\right)\end{array}$ \\
\hline FDG-SUV & $\begin{array}{l}5.1 \pm 1.8 \\
(2.7-8.9)\end{array}$ & $\begin{array}{l}4.8 \pm 1.9 \\
(1.9-7.3)\end{array}$ \\
\hline FDG-SUV ${ }_{\max }$ & $\begin{array}{l}12.1 \pm 5.7 \\
(4.0-23.2)\end{array}$ & $\begin{array}{l}9.7 \pm 4.4 \\
(2.6-15.9)\end{array}$ \\
\hline FDG-HF & $\begin{array}{l}51 \pm 26 \% \\
(8-100 \%)\end{array}$ & $\begin{array}{l}66 \pm 24 \% \\
(34-100 \%)\end{array}$ \\
\hline FDG-HV & $\begin{array}{l}6 \pm 3 \mathrm{~cm}^{3} \\
\left(2-12 \mathrm{~cm}^{3}\right)\end{array}$ & $\begin{array}{l}9.5 \pm 10.7 \mathrm{~cm}^{3} \\
\left(1-36 \mathrm{~cm}^{3}\right)\end{array}$ \\
\hline HX4-SUV $V_{\text {mean }}$ & $\begin{array}{l}0.8 \pm 0.2 \\
(0.5-1.2)\end{array}$ & $\begin{array}{l}0.9 \pm 0.3 \\
(0.5-1.3)\end{array}$ \\
\hline HX4-SUV $\max$ & $\begin{array}{l}1.2 \pm 0.4 \\
(0.6-2.0)\end{array}$ & $\begin{array}{l}1.4 \pm 0.5 \\
(0.6-2.1)\end{array}$ \\
\hline HX4-TMR & $\begin{array}{l}1.6 \pm 0.4 \\
(1.0-2.7)\end{array}$ & $\begin{array}{l}1.9 \pm 0.7 \\
(1.1-2.8)\end{array}$ \\
\hline HX4-HF & $\begin{array}{l}9 \pm 10 \% \\
(0-33 \%)\end{array}$ & $\begin{array}{l}27 \pm 27 \% \\
(0-71 \%)\end{array}$ \\
\hline HX4-HV & $\begin{array}{l}2 \pm 3 \mathrm{~cm}^{3} \\
\left(0-11 \mathrm{~cm}^{3}\right)\end{array}$ & $\begin{array}{l}10 \pm 14 \mathrm{~cm}^{3} \\
\left(0-45 \mathrm{~cm}^{3}\right)\end{array}$ \\
\hline
\end{tabular}

\section{RESULTS}

In this study we analysed the $\left[{ }^{18} \mathrm{~F}\right] \mathrm{FDG}$ and $\left[{ }^{18} \mathrm{~F}\right] \mathrm{HX} 4$ uptake in the primary tumour and lymph nodes of 20 patients with HNSCC before the start of (chemo)radiotherapy. Figure 7.1 and Figure 7.2 provide examples of included patients. We detected tumour hypoxia in $13 / 20$ of the primary tumours and $9 / 12$ of metastatic lymph nodes. The $\left[{ }^{18} \mathrm{~F}\right] \mathrm{HX} 4-\mathrm{HV}$ was therefore absent or smaller than the $\left[{ }^{18} \mathrm{~F}\right]$ FDG-HV for $18 / 20$ primary lesions and $9 / 12$ involved lymph nodes $(p<0.001)$. The average values of the GTV, $\left[{ }^{18} \mathrm{~F}\right] \mathrm{FDG}$ and $\left[{ }^{18} \mathrm{~F}\right] \mathrm{HX} 4$ parameters are shown in Table 7.1. 


\section{Overall correlation of $\left[{ }^{18} \mathrm{~F}\right] \mathrm{FDG}$ and $\left[{ }^{18} \mathrm{~F}\right] \mathrm{HX} 4$ parameters}

Potential correlations between tumour volume, $\left[{ }^{18} \mathrm{~F}\right] \mathrm{FDG}$ and $\left[{ }^{18} \mathrm{~F}\right] \mathrm{HX} 4 \mathrm{PET}$ based parameters were investigated (Table 7.2; Figure 7.3). Combining the parameters from both $\mathrm{GTV}_{\text {prim }}$ and $\mathrm{GTV}_{\text {In }}$ we observed a significant correlation between the volume of the lesion and the $\left[{ }^{18} \mathrm{~F}\right] \mathrm{HX} 4$ parameters $\mathrm{SUV}_{\max }(\mathrm{R}=0.39, \mathrm{P}=0.03), \mathrm{TMR}_{\max }(\mathrm{R}=0.62, \mathrm{P}<0.001),\left[{ }^{18} \mathrm{~F}\right] \mathrm{HX} 4-$ $\mathrm{HF}(\mathrm{R}=0.52, \mathrm{P}<0.01)$ and $\left[{ }^{18} \mathrm{~F}\right] \mathrm{HX} 4-\mathrm{HV}(\mathrm{R}=0.87, \mathrm{P}<0.001)$. Also all $\left[{ }^{18} \mathrm{~F}\right] \mathrm{FDG}$ parameters were significantly correlated with the tumour volume. A significant correlation was observed between all $\left[{ }^{18} \mathrm{~F}\right] \mathrm{HX} 4$ parameters and the $\left[{ }^{18} \mathrm{~F}\right] \mathrm{FDG}$ SUV ${ }_{\text {mean }}, \mathrm{SUV}_{\max }$ and $\left[{ }^{18} \mathrm{~F}\right] \mathrm{FDG}-\mathrm{HV}$, with the most pronounced correlation between $\left[{ }^{18} \mathrm{~F}\right] \mathrm{HX} 4-\mathrm{HV}$ and $\left[{ }^{18} \mathrm{~F}\right] \mathrm{FDG}-\mathrm{HV} \quad(\mathrm{R}=0.93$, $\mathrm{P}<0.001)$.

Table 7.2: Pearson's correlation coefficient (R) and corresponding p-values of the $\left[{ }^{18} F\right] F D G$ and $\left[{ }^{18} F\right] H X 4$ PET parameters from both the primary lesions and involved lymph nodes.

\begin{tabular}{|c|c|c|c|c|c|}
\hline & Volume GTV & FDG -SUV ${ }_{\text {mean }}$ & FDG-SUV $_{\max }$ & FDG-HF & FDG-HV \\
\hline \multicolumn{6}{|c|}{ Volume GTV } \\
\hline $\mathrm{R}$ & - & 0.39 & 0.50 & -0.56 & 0.88 \\
\hline$p$ & - & 0.03 & $<0.01$ & $<0.001$ & $<0.001$ \\
\hline \multicolumn{6}{|c|}{ HX4-SUV $V_{\text {mean }}$} \\
\hline $\mathrm{R}$ & 0.21 & 0.55 & 0.41 & -0.08 & 0.41 \\
\hline$p$ & 0.25 & 0.001 & 0.02 & 0.67 & 0.02 \\
\hline \multicolumn{6}{|c|}{ HX4-SUV $V_{\max }$} \\
\hline $\mathrm{R}$ & 0.39 & 0.56 & 0.54 & -0.31 & 0.46 \\
\hline$p$ & 0.03 & $<0.001$ & $<0.01$ & 0.09 & $<0.01$ \\
\hline \multicolumn{6}{|c|}{ HX4-TMR $\max$} \\
\hline$R$ & 0.62 & 0.61 & 0.59 & -0.42 & 0.66 \\
\hline$P$ & $<0.001$ & $<0.001$ & $<0.001$ & 0.02 & $<0.001$ \\
\hline \multicolumn{6}{|c|}{ HX4-HF } \\
\hline$R$ & 0.52 & 0.59 & 0.38 & -0.12 & 0.73 \\
\hline$P$ & $<0.01$ & $<0.001$ & 0.03 & 0.52 & $<0.001$ \\
\hline \multicolumn{6}{|c|}{ HX4-HV } \\
\hline$R$ & 0.87 & 0.41 & 0.36 & -0.25 & 0.93 \\
\hline$p$ & $<0.001$ & 0.02 & 0.05 & 0.16 & $<0.001$ \\
\hline
\end{tabular}

GTV = Gross tumour volume, SUV = standardized uptake value, TMR = Tumor to muscle ratio, $\mathrm{HF}=$ high uptake fraction, $\mathrm{HV}=$ high uptake volume 

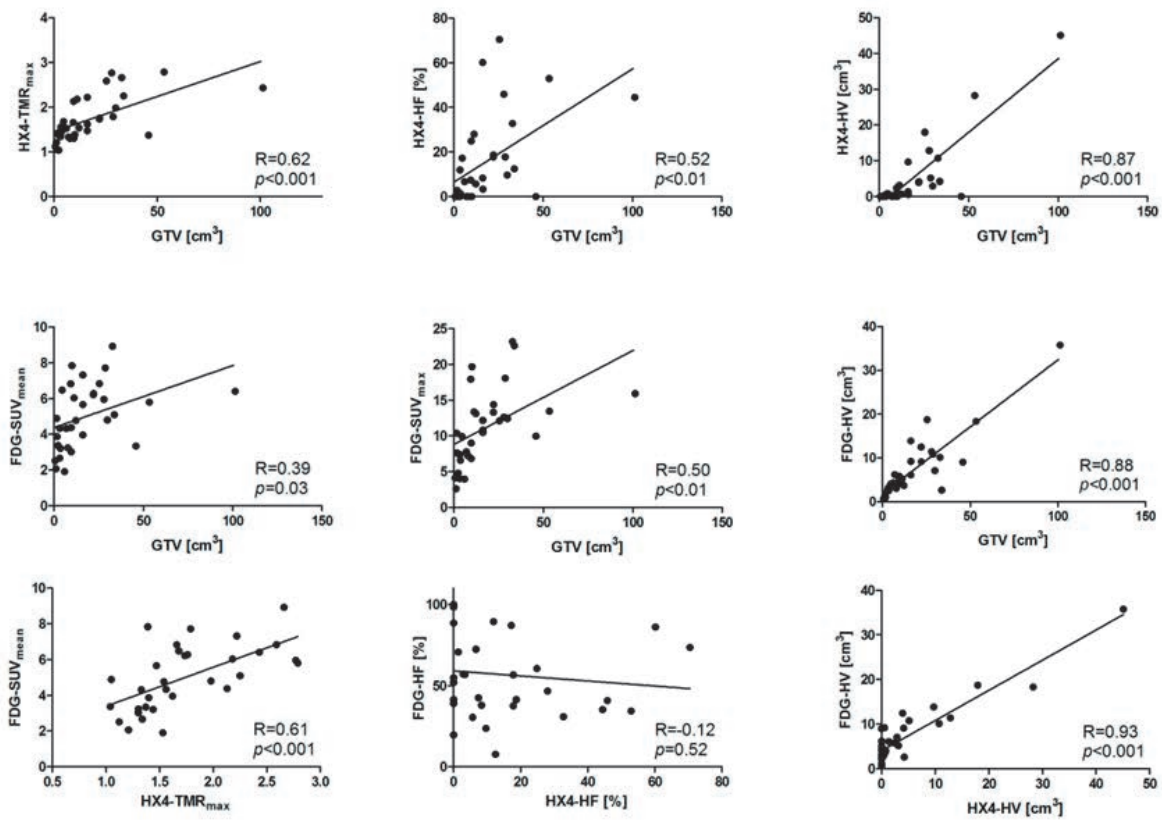

Figure 7.3: Correlation plots of the relationship between the gross tumour volume (GTV), FDG and HX4 uptake parameters.

\section{Spatial relationship between $\left[{ }^{18} \mathrm{~F}\right] \mathrm{FDG}$ and $\left[{ }^{18} \mathrm{~F}\right] \mathrm{HX} 4$ uptake}

A visual representation of the $\left[{ }^{18} \mathrm{~F}\right] \mathrm{FDG}$ and $\left[{ }^{18} \mathrm{~F}\right] \mathrm{HX} 4$ high uptake fractions for each individual patient are given in Figure $7.4\left(\mathrm{GTV}_{\text {prim }}\right)$ and Figure S7.1 $\left(\mathrm{GTV}_{\mathrm{In}}\right)$.

Of the $20 \mathrm{GTV}_{\text {prim, }}$ seven lesions showed no tumour hypoxia. In four lesions, the $\left[{ }^{18} \mathrm{~F}\right] \mathrm{HX} 4$ $\mathrm{HV}$ was entirely located within the $\left[{ }^{18} \mathrm{~F}\right] \mathrm{FDG}-\mathrm{HV}$, whereas for the remaining nine lesions a partial mismatch between the $\left[{ }^{18} \mathrm{~F}\right] \mathrm{HX} 4$ and $\left[{ }^{18} \mathrm{~F}\right] \mathrm{FDG}$ high uptake volumes was found. In these nine lesions, $25 \pm 21 \%$ (range: $5-64 \%$ ) of the HX4-HV was located outside the FDG-HV.

Of the 12 metastatic lymph nodes, three showed no tumour hypoxia, in three other lesions the $\left[{ }^{18} \mathrm{~F}\right] \mathrm{HX} 4-\mathrm{HV}$ was within the $\left[{ }^{18} \mathrm{~F}\right] \mathrm{FDG}-\mathrm{HV}$, whereas for the other six lymph node regions a partial mismatch was observed. In these six lesions, $21 \pm 11 \%$ (range: $6-37 \%$ ) of the HX4-HV was located outside the FDG-HV.

To compare the spatial distribution of the tracer uptake, an uptake level above background is essential in both PET scans. Therefore the results of the voxel-wise comparison and DICE calculations are provided only for the lesions with observed tumour hypoxia $\left(G_{\text {prim }} ; \mathrm{N}=13, \mathrm{GTV}_{\text {In }} ; \mathrm{N}=9\right)$ ). The voxel-wise comparison of the $\left[{ }^{18} \mathrm{~F}\right] \mathrm{HX} 4$ and $\left[{ }^{18} \mathrm{~F}\right] \mathrm{FDG}$ uptake within the GTVs showed a large diversity for the primary lesion $(R=0.47 \pm 0.31$, range -0.04 to 0.85 ; Figure $S 7.2$ ), whereas a good correlation was observed for the lymph nodes $(0.73 \pm 0.12$ range: 0.59 to 0.91$)$. For $\mathrm{GTV}_{\text {prim }}$ the average DICE similarity coefficient was $0.38 \pm 0.22$, with a range from 0.04 (Patient 7) to 0.78 (Patient 11; Figure 7.4). For 
$\mathrm{GTV}_{\text {In }}$ the average DICE was $0.57 \pm 0.32$, with a range from 0.10 (Patient 11 ) to 0.88 (Patient 5; Figure S7.1). Note that the small DICE coefficients were observed due to large differences in $\left[{ }^{18} \mathrm{~F}\right] \mathrm{HX} 4$ and $\left[{ }^{18} \mathrm{~F}\right] \mathrm{FDG}$ high volumes.

For the hypoxic lesions, we observed no relationship between the interval of both scans and the voxel wise correlation coefficients for $\operatorname{GTV}_{\text {prim }}(R=-0.11, p=0.72)$ and $\operatorname{GTV}_{\text {In }}(R=-$ $0.46, p=0.22$ ). Also no correlation was observed between the time interval and the DICE of $\mathrm{GTV}_{\text {prim }}(\mathrm{R}=0.17, \mathrm{p}=0.57)$ however there was a significant correlation between the time interval and the DICE coefficient of $\operatorname{GTV}_{\ln }(R=-0.83, p<0.01)$

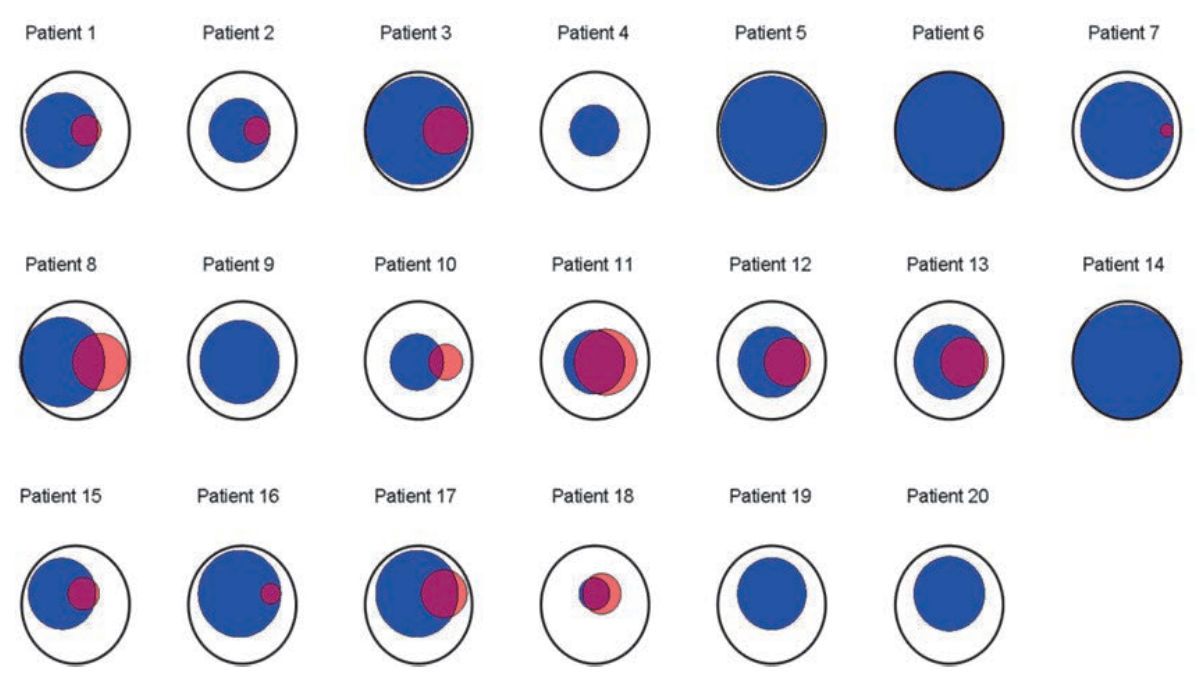

Figure 7.4: Visual representation of the overlap of $\left[{ }^{18} \mathrm{~F}\right] \mathrm{FDG}$ high fraction (blue) and $\left[{ }^{18} \mathrm{~F}\right] \mathrm{HX} 4$ high fraction (red) of the primary tumour (black) of all patients. The range of the $\left[{ }^{18} \mathrm{~F}\right]$ FDG high fraction is from $8 \%$ (patient 18), to $100 \%$ (patient 6). The range of the $\left[{ }^{18} \mathrm{~F}\right] \mathrm{HX} 4$ high fraction is from $0 \%$ (patient 4, 5, 6, 9, 14,19 and 20) to 33\% (patient 11). 


\section{DISCUSSION}

In this study we characterized the relationship between the PET tracers $\left[{ }^{18} \mathrm{~F}\right] \mathrm{FDG}$ (glucose metabolism) and $\left[{ }^{18} \mathrm{~F}\right] \mathrm{HX} 4$ (hypoxia) in patients with HNSCC. First, the relationship between the general $\left[{ }^{18} \mathrm{~F}\right] \mathrm{FDG}$ and $\left[{ }^{18} \mathrm{~F}\right] \mathrm{HX} 4$ uptake parameters were evaluated. Second, we assessed the similarity in spatial uptake pattern between $\left[{ }^{18} \mathrm{~F}\right] \mathrm{FDG}$ and $\left[{ }^{18} \mathrm{~F}\right] \mathrm{HX} 4$ PET imaging.

On a general tumour level we observed a relationship between tumour volume, $\left[{ }^{18} \mathrm{~F}\right] \mathrm{FDG}$ and $\left[{ }^{18} \mathrm{~F}\right] \mathrm{HX} 4$ uptake parameters. These results are in agreement with the previous published research, which also observed a moderate correlation between $\left[{ }^{18} \mathrm{~F}\right] \mathrm{FMISO}$ PET and $\left[{ }^{18} \mathrm{~F}\right]$ FDG PET uptake parameters $(17,18)$. Conversely, no correlation between $\left[{ }^{18} \mathrm{~F}\right]$ FDG PET imaging and $\mathrm{pO}_{2}$ polarography or $\mathrm{HIF-1} \alpha$ staining was reported previously $(17,18)$. Therefore, although we found a correlation between $\left[{ }^{18} \mathrm{~F}\right] \mathrm{FDG}$ and $\left[{ }^{18} \mathrm{~F}\right] \mathrm{HX} 4$ PET uptake, they both represent different biological properties of tumours, which can be useful to predict treatment response. Several studies in patients with head and neck cancer have shown that hypoxia PET imaging is superior to $\left[{ }^{18}\right.$ F]FDG PET imaging for the prediction of treatment response $(18,19)$. However, Thorwarth et al. (20) showed that a combination of both imaging modalities has the highest potential to predict treatment success.

In our study we observed that in the majority of HNSCC patients the hypoxic volume was smaller than the high metabolic tumour volume. This is in agreement with the results we previously reported in patients with NSCLC (8). This might allow a radiotherapy dose escalation to the smaller hypoxic tumour volume. At the moment an ongoing randomized phase III clinical study 'adaptive radiation treatment for head and neck cancer' (ARTFORCE; NCT01504815), investigates the effect of an $\left[{ }^{18}\right.$ F]FDG PET based radiation dose redistribution, on the locoregional control in patients with HNSCC. In addition, several radiotherapy planning studies have shown that it is technically feasible to perform radiotherapy dose escalation based on hypoxia PET imaging without increasing the dose to the normal tissue (21-23). Nevertheless, the most essential knowledge we need, is whether the hypoxic or metabolic volume is related to recurrences after treatment. This might indicate whether the hypoxic, metabolic or a combination of both volumes should be used in the adaptation of the radiation dose, with the aim to maximize the therapeutic ratio for each individual patient (24). Dirix et al. (25) showed for example that all recurrences after chemoradiotherapy $(N=9)$ were located within the high metabolic regions, however three of these recurrences were located outside the hypoxic volume. Due et al. (10), however, report that only $54 \%$ of the recurrences were located within the visually defined high metabolic area, while $96 \%$ of the recurrences were located within the clinical target volume (CTV; GTV with a $1 \mathrm{~cm}$ margin). This literature evidence prompted us to be careful redistributing the dose within the CTV. Therefore, also alternative methods to decrease tumour hypoxia by the aid of hypoxia targeting or hypoxia-modification should be further explored. In these studies hypoxia PET imaging could be used to monitor the response to this treatment and stratify patients based on their pre-treatment hypoxic status.

This study has several limitations. First, there was a time interval between the $\left[{ }^{18}\right.$ F]FDG 
and $\left[{ }^{18} \mathrm{~F}\right] \mathrm{HX} 4 \mathrm{PET} / \mathrm{CT}$ scans, in which changes in tumour metabolism or hypoxia may have occurred. We observed a relationship between the time interval and DICE coefficient of the GTV $_{\text {In }}$, which might indicate that a shorter time interval could improve the spatial correlation between the $\left[{ }^{18} \mathrm{~F}\right] \mathrm{FDG}$ and $\left[{ }^{18} \mathrm{~F}\right] \mathrm{HX} 4$ uptake. However, this was not observed for the voxel-wise correlation coefficients. For most of the patients the time interval was small (median: seven days) and no interventions were performed between both scans. Second, small lesions were also included in the analysis. Small lesions are prone to present only a limited amount of tumour hypoxia. In addition, in these lesions the partial volume effect plays a larger role, causing an underestimation of the absolute uptake. This might influence the correlation between the tumour volume and the $\left[{ }^{18} \mathrm{~F}\right] \mathrm{HX} 4$ and $\left[{ }^{18} \mathrm{~F}\right] \mathrm{FDG}$ uptake measurements. Third, the thresholds to define a $\left[{ }^{18} \mathrm{~F}\right] \mathrm{HX} 4$ or $\left[{ }^{18} \mathrm{~F}\right] \mathrm{FDG}$ high uptake were defined based on previous literature and ongoing clinical trials, a change in this definition will influence the results. However, based on the results of our previous study in patients with NSCLC, we can state that the mismatch between high $\left[{ }^{18} \mathrm{~F}\right] \mathrm{HX} 4$ and high $\left[{ }^{18} \mathrm{~F}\right]$ FDG volumes is relative stable for different thresholds (8).

In conclusion, there is a positive correlation between $\left[{ }^{18} \mathrm{~F}\right] \mathrm{HX} 4$ and $\left[{ }^{18} \mathrm{~F}\right] \mathrm{FDG}$ uptake parameters on a global tumour level. On average, the $\left[{ }^{18} \mathrm{~F}\right] \mathrm{HX} 4-\mathrm{HV}$ is smaller than the $\left[{ }^{18} \mathrm{~F}\right]$ FDG-HV . In the majority of lesions a partial mismatch between the $\left[{ }^{18} \mathrm{~F}\right] \mathrm{HX} 4$ and $\left[{ }^{18}\right.$ F]FDG high uptake volumes was observed, therefore $\left[{ }^{18} \mathrm{~F}\right]$ FDG PET imaging cannot be used as a surrogate for hypoxia. $\left[{ }^{18} \mathrm{~F}\right] \mathrm{HX} 4$ PET imaging provides complementary information to $\left[{ }^{18}\right.$ F]FDG PET imaging.

\section{ACKNOWLEDGEMENTS}

The authors like to thank the patients who agreed to participate to this study and the PETCT group, data-management (Anita Botterweck) and trial-poli (Claudia Offermann and John Paulissen) of MAASTRO clinic for their contribution to the data acquisition. Authors acknowledge financial support from the QuIC-ConCePT project, which is partly funded by EFPI A companies and the Innovative Medicine Initiative Joint Undertaking (IMI JU) under Grant Agreement No. 115151. Authors also acknowledge financial support from EU 7th framework program (EURECA, ARTFORCE - $n^{\circ}$ 257144, REQUITE - $n^{\circ}$ 601826), Kankeronderzoekfonds Limburg from the Health Foundation Limburg and the Dutch Cancer Society (KWF MAC 2011-4970). 
146 |Chapter 7

\section{SUPPLEMENTARY DATA}

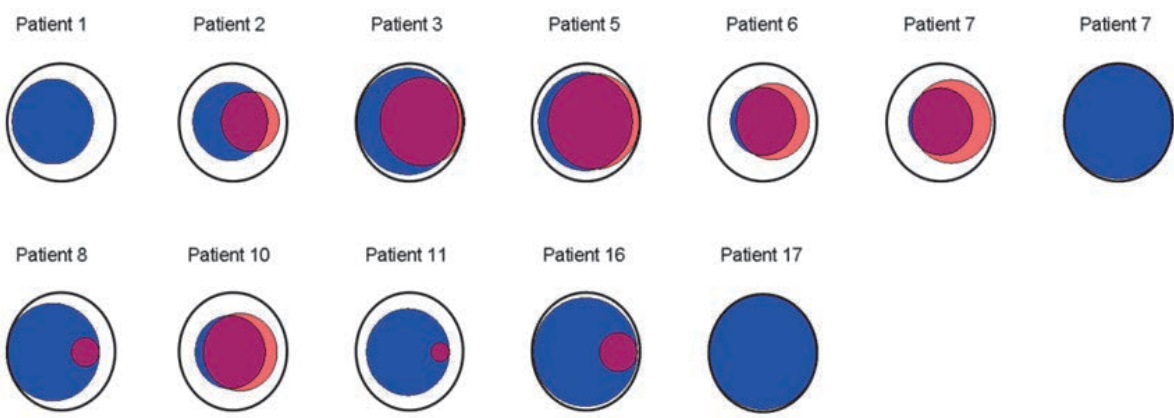

Figure S7.1: Visual representation of the overlap of $\left[{ }^{18} \mathrm{~F}\right] F D G$ high fraction (blue) and $\left[{ }^{18} \mathrm{~F}\right] \mathrm{HX} 4$ high fraction (red) for the metastatic lymph nodes (black) of all patients.
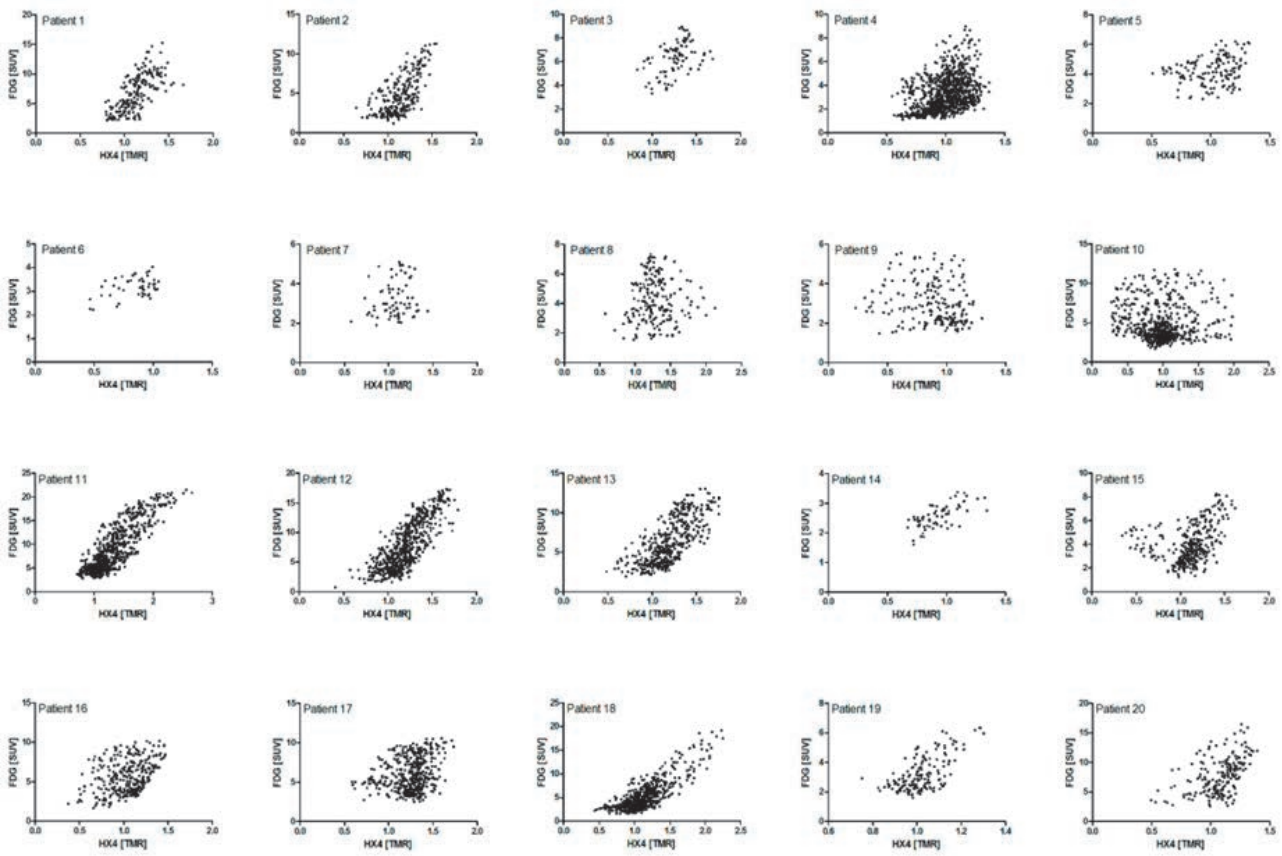

Figure S7.2: Voxel wise comparison of the $\left[^{18} \mathrm{~F}\right] \mathrm{HX} 4$ (tumor-muscle-ratio) and $\left[^{18} \mathrm{~F}\right] \mathrm{FDG}$ PET (SUV) uptake within the primary lesions. 


\section{REFERENCES}

1. Harris AL. Hypoxia--a key regulatory factor in tumour growth. Nat Rev Cancer. 2002;2:38-47.

2. Horsman MR, Mortensen LS, Petersen JB, Busk M, Overgaard J. Imaging hypoxia to improve radiotherapy outcome. Nat Rev Clin Oncol. 2012;9:674-87.

3. Troost EG, Schinagl DA, Bussink J, Boerman OC, van der Kogel AJ, Oyen WJ, et al. Innovations in radiotherapy planning of head and neck cancers: role of PET. J Nucl Med. 2010;51:66-76.

4. Peeters SG, Zegers CM, Yaromina A, Van Elmpt W, Dubois L, Lambin P. Current preclinical and clinical applications of hypoxia PET imaging using 2-nitroimidazoles. $\mathrm{Q}$ J Nucl Med Mol Imaging. 2015;59:39-57.

5. Zips D, Zophel K, Abolmaali N, Perrin R, Abramyuk A, Haase R, et al. Exploratory prospective trial of hypoxia-specific $\mathrm{PET}$ imaging during radiochemotherapy in patients with locally advanced head-and-neck cancer. Radiother Oncol. 2012;105:21-8.

6. Dubois LJ, Lieuwes NG, Janssen MH, Peeters WJ, Windhorst AD, Walsh JC, et al. Preclinical evaluation and validation of [18F]HX4, a promising hypoxia marker for PET imaging. Proc Natl Acad Sci U S A. 2011;108:14620-5.

7. Peeters SG, Zegers CM, Lieuwes NG, van Elmpt W, Eriksson J, van Dongen GA, et al. A Comparative Study of the Hypoxia PET Tracers [(18)F]HX4, [(18)F]FAZA, and [(18)F]FMISO in a Preclinical Tumor Model. Int J Radiat Oncol Biol Phys. 2015;91:351-9.

8. Zegers CM, van Elmpt W, Reymen B, Even AJ, Troost EG, Ollers MC, et al. In vivo quantification of hypoxic and metabolic status of NSCLC tumors using [18F]HX4 and [18F]FDG-PET/CT imaging. Clin Cancer Res. 2014;20:6389-97.

9. Aerts HJ, van Baardwijk AA, Petit SF, Offermann C, Loon J, Houben R, et al. Identification of residual metabolic-active areas within individual NSCLC tumours using a pre-radiotherapy (18)Fluorodeoxyglucose-PET-CT scan. Radiother Oncol. 2009;91:386-92.

10. Due AK, Vogelius IR, Aznar MC, Bentzen SM, Berthelsen AK, Korreman SS, et al. Recurrences after intensity modulated radiotherapy for head and neck squamous cell carcinoma more likely to originate from regions with high baseline [18F]-FDG uptake. Radiother Oncol. 2014;111:360-5.

11. Gatenby RA, Gillies RJ. Why do cancers have high aerobic glycolysis? Nat Rev Cancer. 2004;4:891-9.

12. Vander Heiden MG, Cantley LC, Thompson CB. Understanding the Warburg effect: the metabolic requirements of cell proliferation. Science. 2009;324:1029-33.

13. van Loon J, Janssen $M H$, Ollers $M$, Aerts $H J$, Dubois $L$, Hochstenbag $M$, et al. PET imaging of hypoxia using [18F]HX4: a phase I trial. Eur J Nucl Med Mol Imaging. 2010;37:1663-8.

14. Boellaard R, Oyen WJ, Hoekstra CJ, Hoekstra OS, Visser EP, Willemsen AT, et al. The Netherlands protocol for standardisation and quantification of FDG whole body PET studies in multi-centre trials. European journal of nuclear medicine and molecular imaging. 2008;35:2320-33.

15. Heukelom J, Hamming O, Bartelink H, Hoebers F, Giralt J, Herlestam T, et al. Adaptive and innovative Radiation Treatment FOR improving Cancer treatment outcomE 
(ARTFORCE); a randomized controlled phase II trial for individualized treatment of head and neck cancer. BMC Cancer. 2013;13:84.

16. van Elmpt W, De Ruysscher D, van der Salm A, Lakeman A, van der Stoep J, Emans D, et al. The PET-boost randomised phase II dose-escalation trial in non-small cell lung cancer. Radiother Oncol. 2012;104:67-71.

17. Zimny M, Gagel B, DiMartino E, Hamacher K, Coenen HH, Westhofen M, et al. FDG--a marker of tumour hypoxia? A comparison with [18F]fluoromisonidazole and pO2polarography in metastatic head and neck cancer. Eur J Nucl Med Mol Imaging. 2006;33:1426-31.

18. Sato J, Kitagawa $\mathrm{Y}$, Yamazaki $\mathrm{Y}$, Hata H, Okamoto S, Shiga T, et al. 18F-fluoromisonidazole PET uptake is correlated with hypoxia-inducible factor-1alpha expression in oral squamous cell carcinoma. J Nucl Med. 2013;54:1060-5.

19. Komar G, Lehtio K, Seppanen M, Eskola O, Levola H, Lindholm P, et al. Prognostic value of tumour blood flow, [(1)(8)F]EF5 and [(1)(8)F]FDG PET/CT imaging in patients with head and neck cancer treated with radiochemotherapy. Eur J Nucl Med Mol Imaging. 2014;41:2042-50.

20. Thorwarth D, Eschmann SM, Holzner F, Paulsen F, Alber M. Combined uptake of [18F]FDG and [18F]FMISO correlates with radiation therapy outcome in head-andneck cancer patients. Radiother Oncol. 2006;80:151-6.

21. Hoeben BA, Bussink J, Troost EG, Oyen WJ, Kaanders JH. Molecular PET imaging for biology-guided adaptive radiotherapy of head and neck cancer. Acta Oncol. 2013;52:1257-71.

22. Toma-Dasu I, Uhrdin J, Antonovic L, Dasu A, Nuyts S, Dirix P, et al. Dose prescription and treatment planning based on FMISO-PET hypoxia. Acta Oncol. 2012;51:222-30.

23. Chang JH, Wada M, Anderson NJ, Lim Joon D, Lee ST, Gong SJ, et al. Hypoxia-targeted radiotherapy dose painting for head and neck cancer using (18)F-FMISO PET: a biological modeling study. Acta Oncol. 2013;52:1723-9.

24. Grau C, Hoyer M, Alber M, Overgaard J, Lindegaard JC, Muren LP. Biology-guided adaptive radiotherapy (BiGART)--more than a vision? Acta Oncol. 2013;52:1243-7.

25. Dirix P, Vandecaveye V, De Keyzer F, Stroobants S, Hermans R, Nuyts S. Dose painting in radiotherapy for head and neck squamous cell carcinoma: value of repeated functional imaging with (18)F-FDG PET, (18)F-fluoromisonidazole PET, diffusionweighted MRI, and dynamic contrast-enhanced MRI. J Nucl Med. 2009;50:1020-7. 


\section{CHAPTER}

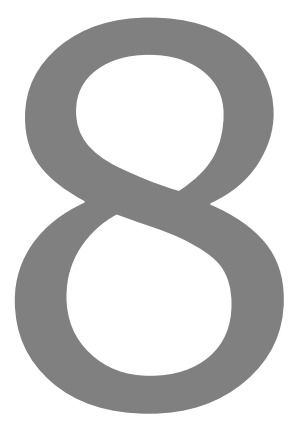

Evaluation of tumour hypoxia during radiotherapy using $\left[{ }^{18} \mathrm{~F}\right] \mathrm{HX} 4 \mathrm{PET}$ imaging and blood biomarkers in patients with head and neck cancer

Catharina M.L. Zegers, Frank J.P. Hoebers, Wouter van Elmpt, Judith A. Bons, Michel C. Öllers, Esther G.C. Troost, Daniëlle Eekers, Leo Balmaekers, Marlies Arts-Pechtold, Felix M. Mottaghy, Philippe Lambin 


\section{ABSTRACT}

Background and Purpose: Increased tumour hypoxia is associated with a worse overall survival in patients with head and neck squamous cell carcinoma (HNSCC). The aims of this study were to evaluate treatment-associated changes in $\left[{ }^{18} \mathrm{~F}\right] \mathrm{HX} 4-\mathrm{PET}$, hypoxia-related blood biomarkers, and their interdependence.

Material and Methods: $\left[{ }^{18} \mathrm{~F}\right] \mathrm{HX} 4-\mathrm{PET} / \mathrm{CT}$ scans of 20 patients with HNSCC were acquired at baseline and after $\pm 20 \mathrm{~Gy}$ of radiotherapy. Within the gross-tumour-volumes (GTV; primary and lymph nodes), mean and maximum standardized uptake values, the hypoxic fraction (HF) and volume (HV) were calculated. Also, the changes in spatial uptake pattern were evaluated using $\left[{ }^{18} \mathrm{~F}\right] \mathrm{HX} 4-\mathrm{PET} / \mathrm{CT}$ imaging. Of all patients, the plasma concentration of CAIX, osteopontin and VEGF was assessed.

Results: At baseline tumour hypoxia was detected in 69\% (22/32) of the GTVs. During therapy, we observed a significant decrease in all image parameters. The HF decreased from $21.7 \pm 19.8 \%$ (baseline) to $3.6 \pm 10.0 \%$ (during treatment; $P<0.001$ ). Only two patients had a $\mathrm{HV}>1 \mathrm{~cm}^{3}$ during treatment which was located for $>98 \%$ within the baseline HV. During treatment no significant changes in plasma CAIX or VEGF were observed, while osteopontin was increased.

Conclusions: $\left[{ }^{18} \mathrm{~F}\right] \mathrm{HX} 4-\mathrm{PET} / \mathrm{CT}$ imaging allows monitoring changes in hypoxia during (chemo)radiotherapy whereas the blood biomarkers were not able to detect a treatmentassociated decrease in hypoxia. 


\section{INTRODUCTION}

Tumour cell hypoxia is known to promote resistance to cancer treatment, to increase tumour aggressiveness, and to be a prognostic factor for survival (1). Non-invasive imaging of tumour hypoxia by means of positron emission tomography (PET) has been shown to predict loco-regional control and survival, and may be used to select patients for additional anti-hypoxia therapy (2-4). In addition, PET imaging can be used to monitor the response to treatment. Previous studies using the hypoxia PET tracer [18F]FMISO and the metabolic PET tracer [18F]FDG observed that the uptake changes (early) during (chemo)radiotherapy had a higher predictive value than pre-treatment measurements (3, 5). Previously, Overgaard (6) showed that the modification of hypoxia during radiotherapy results in better loco-regional control and survival in patients with a squamous cell carcinoma of the head and neck (HNSCC). However, stratifying patients undergoing ARCON (accelerated radiotherapy with carbogen and nicotinamide) based on their pretherapeutic hypoxic status (pimonidazole staining) demonstrated that the benefit in locoregional control was specifically observed for patients with initial tumour hypoxia before the start of treatment $(7,8)$. The pimonidazole staining of a biopsy, however can only provide information about local hypoxia before the start of treatment. Non-invasive hypoxia PET imaging, on the other hand, provides to opportunity to perform repeated tumour hypoxia measurements in 3-dimensions. Therefore hypoxia PET measurements may be used to select patients likely to have a benefit of additional anti-hypoxia therapy.

One of the hypoxia PET tracers to visualize and quantify tumour hypoxia is the 2nitroimidazole3-[18F]fluoro-2-(4-((2-nitro-1H-imidazol-1-yl)methyl)-1H-1,2,3-triazol-1-

yl)propan-1-ol, [18F]HX4. In previous pre-clinical studies [18F]HX4 was validated as a hypoxia tracer and the repeatability of the tracer uptake was assessed $(9,10)$. In addition, in patients with non-small cell lung cancer (NSCLC), [18F]HX4 showed promising results and was shown to provide additional value to the metabolic PET tracer [18F]FDG $(11,12)$. In this paper, we will investigate the potential of this tracer to detect treatmentassociated changes in hypoxic tumour status in patients with HNSCC.

Another method to obtain information on tumour hypoxia may be the measurement of hypoxia-related proteins or enzymes in plasma. Potential relevant hypoxia markers are plasma osteopontin, carbonic anhydrase IX (CAIX) and vascular endothelial growth factor (VEGF). Osteopontin is activated under hypoxic conditions and is inversely correlated with the $\mathrm{PO} 2$ value of the tumour (13). In addition plasma osteopontin is a significant predictor for the response to radiotherapy in patients with head and neck cancer (14). CAIX expression is upregulated under the influence of tumour hypoxia. Also, in patients with NSCLC a high level of plasma CAIX was associated with a shorter overall survival (15). Last, hypoxia activates hypoxia inducible factor (HIF-1) which induces a high expression of VEGF, the primary cytokine related to angiogenesis. VEGF may therefore serve as an indirect marker of tumour hypoxia. These three markers might have the potential to stratify patients based on their hypoxic tumour status. 
The aims of this study were to evaluate the changes in hypoxia during treatment in patients with HNSCC, the spatial stability of the uptake pattern and the presence of plasma osteopontin, CAIX and VEGF in relationship to hypoxia imaging.

\section{MATERIALS AND METHODS}

\section{Patients}

Between January 2012 and October 2014 we included 20 patients in this imaging study. Patient, tumour and treatment characteristics are shown in Table 8.1. The study was approved by the Ethical Review Committee of Maastricht University Medical Centre and registered on clinicaltrial.gov (NCT01347281). All patients provided written informed consent before study entry.

\section{PET/CT imaging}

$\left[{ }^{18} \mathrm{~F}\right] \mathrm{HX} 4$ was produced as described in previous publications $(9,16-18)$. After intravenous administration of an average $( \pm \mathrm{SD})$ dose of $378 \pm 84 \mathrm{MBq}\left[{ }^{18} \mathrm{~F}\right] \mathrm{HX} 4, \mathrm{PET} / \mathrm{CT}$ imaging was performed at $1.5 \mathrm{~h}, 3 \mathrm{~h}$ and $4 \mathrm{~h}$ post-injection (p.i.) for 15,15 and 20 minutes, respectively for a single bed position centred around the primary tumour. After 10 patients, an interim analysis showed highest contrast at the imaging time-point at $4 \mathrm{~h} \mathrm{p.i.,} \mathrm{and} \mathrm{this} \mathrm{was} \mathrm{used}$ from then onwards.

$\left[{ }^{18} \mathrm{~F}\right] \mathrm{HX} 4$ PET/CT scans were acquired before the start of external beam radiotherapy and during the radiation treatment; after an average ( \pm SD) dose of $21 \pm 2$ Gy using a Biograph $40 \mathrm{PET} / \mathrm{CT}$ scanner (Siemens Healthcare, Erlangen, Germany). Scatter and attenuation corrections were applied, PET images were reconstructed using OSEM 2D (Ordered Subset Expectation Maximization, 4 iterations, 8 subsets) and a Gaussian filter of $5 \mathrm{~mm}$. Imaging was performed in radiotherapy position, with the patient positioned on a flat table top using an immobilization mask and a movable laser alignment system.

\section{Image analysis}

The gross tumour volume of the primary lesion ( $\mathrm{GTV}_{\text {prim}}$ ) and involved lymph nodes $\left(G_{\text {In }}\right)$, were delineated on the $\left[{ }^{18}\right.$ F]FDG PET/CT, used for radiotherapy planning purposes, by two experienced radiation oncologists in consensus. These contours were copied to the $\left[{ }^{18} \mathrm{~F}\right] \mathrm{HX} 4 \mathrm{PET}$ scans by rigid registration. Maximum and mean standardized uptake values $\left(S_{U} V_{\text {max }}\right.$ and $\left.S_{\text {mean }}\right)$, were determined within the GTVs. In addition, the maximum tumour-to-muscle ratio $\left(T M R_{\max }\right)$, defined as the $S U V_{\max }$ in the tumour divided by the SU$V_{\text {mean }}$ in the trapezius muscles was calculated. The hypoxic fraction (HF) and hypoxic volume (HV) were defined as the fraction or volume of the GTV with a TMR larger than 1.4 . 
Table 8.1: Patient, tumour and treatment characteristics

\begin{tabular}{|c|c|c|}
\hline & $\mathbf{N}$ & $\%$ \\
\hline \multicolumn{3}{|l|}{ Gender } \\
\hline Male & 17 & 85 \\
\hline Female & 3 & 15 \\
\hline \multicolumn{3}{|l|}{ Pathology } \\
\hline Squamous cell carcinoma & 20 & 100 \\
\hline \multicolumn{3}{|l|}{ Tumour site } \\
\hline Oropharynx & 7 & 35 \\
\hline Larynx & 8 & 40 \\
\hline Hypopharynx & 5 & 25 \\
\hline \multicolumn{3}{|l|}{ HPV status (Oropharynx) } \\
\hline Positive & 3 & 43 \\
\hline Negative & 3 & 43 \\
\hline Unknown & 1 & 14 \\
\hline \multicolumn{3}{|l|}{ cT-Stage } \\
\hline T1 & 1 & 5 \\
\hline $\mathrm{T} 2$ & 6 & 30 \\
\hline T3 & 11 & 55 \\
\hline T4 & 2 & 10 \\
\hline \multicolumn{3}{|l|}{ cN-Stage } \\
\hline NO & 9 & 45 \\
\hline N1 & 3 & 15 \\
\hline $\mathrm{N} 2 \mathrm{a}$ & 1 & 5 \\
\hline $\mathrm{N} 2 \mathrm{~b}$ & 7 & 35 \\
\hline \multicolumn{3}{|l|}{ Stage grouping } \\
\hline Stage II & 2 & 10 \\
\hline Stage III & 8 & 40 \\
\hline Stage IV & 10 & 50 \\
\hline \multicolumn{3}{|l|}{ Treatment } \\
\hline Radiotherapy & 6 & 30 \\
\hline Cisplatin chemo-radiotherapy & 10 & 50 \\
\hline Cetuximab-radiotherapy & 4 & 20 \\
\hline \multicolumn{3}{|l|}{$\left[{ }^{18} \mathrm{~F}\right] \mathrm{HX} 4$ PET imaging } \\
\hline Baseline & 20 & 100 \\
\hline During RT & 17 & 85 \\
\hline \multicolumn{3}{|c|}{ Radiotherapy dose between $\left[{ }^{18} \mathrm{~F}\right] \mathrm{HX} 4$ scans [Gy] } \\
\hline 18 & 3 & 18 \\
\hline 20 & 5 & 29 \\
\hline 22 & 6 & 35 \\
\hline 24 & 2 & 12 \\
\hline 26 & 1 & 6 \\
\hline
\end{tabular}


To evaluate the spatial location of the $\left[{ }^{18} \mathrm{~F}\right] \mathrm{HX} 4$ PET uptake at baseline and during treatment, the $\left[{ }^{18} \mathrm{~F}\right] \mathrm{HX} 4 \mathrm{PET} / \mathrm{CT}$ acquired during radiotherapy was rigidly registered to the baseline $\left[{ }^{18} \mathrm{~F}\right] \mathrm{HX} 4 \mathrm{PET} / \mathrm{CT}$. The rigid transformation was determined by the registration of the CT scans; subsequently the same transformation was applied to the PET scans and GTV to co-register the images. A visual and voxel-wise comparison of the $\left[{ }^{18} \mathrm{~F}\right] \mathrm{HX} 4$ uptake before and during radiotherapy was performed to compare spatial uptake patterns for both GTV $_{\text {prim }}$ and GTV $_{\text {In }}$.

\section{Blood biomarker analysis}

For all patients, blood samples were collected before and during (chemo)radiotherapy on the day of the $\left[{ }^{18} \mathrm{~F}\right] \mathrm{HX} 4 \mathrm{PET} / \mathrm{CT}$ scan. All blood biomarkers were measured in EDTA plasma. Samples were analysed simultaneously in a certified laboratory, using commercially available kits. CAIX was measured by a sandwich-type immunoassay that uses a mouse monocolonal capture antibody (V10) and a biotinylated mouse monocolonal antibody (M75) as detector (Nuclea Diagnostic Laboratories LLC, Cambridge, MA). Osteopontin and VEGF were measured by an ELISA method. A monoclonal antibody specific for osteopontin/VEGF was pre-coated onto the microplate and an enzyme-lined polyclonal antibody specific for osteopontin /VEGF was used as detector (R\&D Systems (Minneapolis, MN).

To compare the plasma hypoxia markers with the $\left[{ }^{18} \mathrm{~F}\right] \mathrm{HX} 4$ uptake in the $\mathrm{GTV}_{\text {prim }}$ and $G_{\text {In }}$, we hypothesize that these markers reflect the uptake in all lesions within one patient. Therefore the image parameters of multiple GTVs were combined, providing one $\mathrm{SUV}_{\max }$ and $\mathrm{TMR}_{\max }$ (the maximum of the present lesions). The hypoxic volumes were summed and for the SUV mean a weighted average was calculated.

\section{Statistical analysis}

For all parameters mean \pm 1 standard deviation (SD) are reported. Non-parametric test were used to determine significant differences in image and blood plasma parameters (Wilcoxon signed rank test) and to evaluate correlations between imaging parameters and blood parameters (Spearman's correlation coefficient; $R_{s}$ ). Linear regressions were performed to quantify the voxel-wise comparison, and a Pearson correlation coefficient $\left(R_{p}\right)$ was calculated. A p-value $<0.05$ was assumed to be statistically significant.

\section{RESULTS}

In this study we analysed the $\left[{ }^{18} \mathrm{~F}\right] \mathrm{HX} 4$ uptake of 20 patients with HNSCC before the start of radiotherapy. For $3 / 20$ patients the $\left[{ }^{18} \mathrm{~F}\right] \mathrm{HX} 4$ PET scan during radiotherapy was not performed due to the patient's preference or health status. Eleven patients had involvement of the lymph nodes $\left(G_{T V} V_{l n}\right)$, which were separately analysed from the primary lesion $\left(G T V_{\text {prim }}\right)$. The average lesion sizes for $\mathrm{GTV}_{\text {prim }}$ was $17.6 \pm 12.3 \mathrm{~cm}^{3}$ (range: $2.4-46.6 \mathrm{~cm}^{3}$ ) and for GTV $22.6 \pm 30.5$ (range: $1.3-105.2 \mathrm{~cm}^{3}$ ). 


\section{$\left[{ }^{18} \mathrm{~F}\right] \mathrm{HX} 4$ PET uptake at baseline}

In the first 10 patients, $\left[{ }^{18} \mathrm{~F}\right] \mathrm{HX} 4 \mathrm{PET} / \mathrm{CT}$ imaging was acquired at $1.5,3 \mathrm{~h}$ and $4 \mathrm{~h}$ p.i. In their lesions (10 GTV prim and $9 \mathrm{GTV}_{\mathrm{In}}$ ) we observed a significant increase in the $T M R_{\max }$ from $1.5 \mathrm{~h}$ $(1.5 \pm 0.3)$ to $3 \mathrm{~h}$ p.i. $(1.7 \pm 0.4 ; \mathrm{P}<0.01)$, and from $3 \mathrm{~h}$ p.i to $4 \mathrm{~h}$ p.i. $(1.8 \pm 0.6 ; \mathrm{P}=0.02)$ (Figure 8.1). Therefore, $\left[{ }^{18} \mathrm{~F}\right] \mathrm{HX} 4 \mathrm{PET} / \mathrm{CT}$ imaging at $4 \mathrm{~h}$ p.i. was selected as the standard and applied as the single imaging timepoint for the remaining patients.

At baseline we observed tumour hypoxia (TMR>1.4 at $4 \mathrm{~h}$ p.i.) in $69 \%$ (22/32) of the $\mathrm{GTV}_{\text {prim }}$ and GTV $\mathrm{V}_{\text {In }}$. For all lesions we observed an average $S U V_{\text {mean }}, \mathrm{SUV}_{\max }$ and $T M R_{\max }$ of $0.8 \pm 0.2,1.3 \pm 0.5$ and $1.7 \pm 0.5$, respectively. The average HF and HV were $16 \pm 20 \%$ and $4.9 \pm 9.6 \mathrm{~cm}^{3}$. All these image parameters were significantly correlated to the volume of the lesion, $S_{\text {mean }}\left(R_{s}=0.38, p=0.03\right)$, $S U V_{\text {max }}\left(R_{s}=0.57, p<0.001\right), T_{\text {max }}\left(R_{s}=0.75, p<0.001\right)$, HF $\left(R_{s}=0.63, p<0.001\right)$ and $H V\left(R_{s}=0.74, p<0.001\right)$.

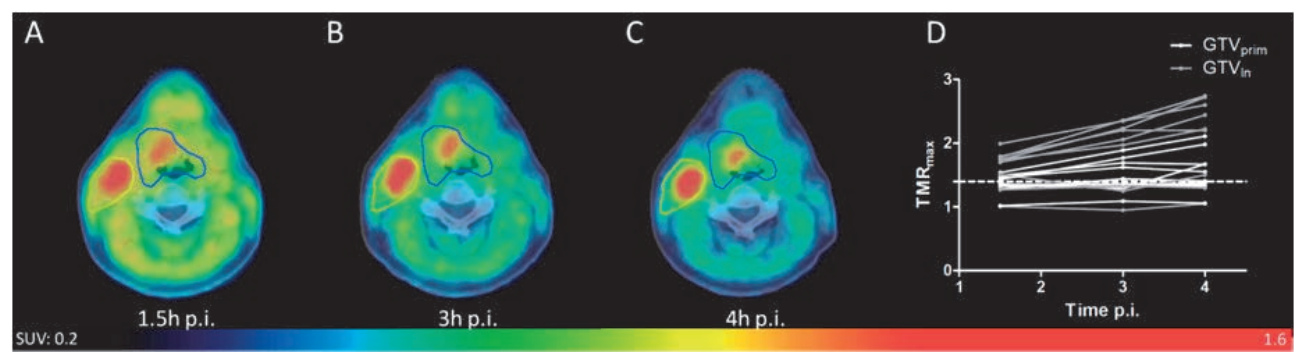

Figure $8.1\left[{ }^{18} \mathrm{~F}\right] H \mathrm{H} 4 \mathrm{PET} / \mathrm{CT}$ scans of a patient with a T2N2bMx squamous cell carcinoma of the oropharynx, scanned at $1.5 \mathrm{~h} \mathrm{~A}), 3 \mathrm{~h} \mathrm{~B}$ ) and $4 \mathrm{~h}$ p.i. C). D) The tumour to muscle ratio (TMR $R_{\max }$ ) for all patients. Shown are the gross tumour volumes of the primary lesions $\left(G T V_{\text {prim }}\right)$ and the metastatic lymph nodes $\left(G T V_{\text {In }}\right)$, which increased significantly (1.5h vs $3 h: p<0.01,3 h$ vs $4 h: p=0.02)$.

\section{$\left[{ }^{18} \mathrm{~F}\right] \mathrm{HX} 4$ PET uptake during treatment}

We observed a significant correlation between the image parameters measured at baseline and during treatment: $S U V_{\text {mean }}\left(R_{s}=0.66, P<0.001\right), S U V_{\text {max }}\left(R_{s}=0.63, P<0.001\right), T_{B} R_{\max }$ $\left(R_{s}=0.57, P<0.01\right), H F\left(R_{s}=0.56, P<0.01\right), H V(R=0.52, P<0.01)$. Taking into account the hypoxic lesions $\left(\mathrm{GTV}_{\text {prim }}\right.$ and $\mathrm{GTV}_{\text {In }}$ ), with a $\left[{ }^{18} \mathrm{~F}\right] \mathrm{HX} 4 \mathrm{PET} / \mathrm{CT}$ scan at baseline and during treatment $(\mathrm{N}=17)$, we observed a significant decrease in all image derived parameters during therapy (Table 8. 2; Figure 8.2). This decrease was independent of the given treatment (Table S8.1). Of the 17 hypoxic lesions at baseline, only 7 had a HF>0 during treatment. In the other 10 lesions hypoxia as measured by $\left[{ }^{18} \mathrm{~F}\right] \mathrm{HX} 4$ PET imaging had disappeared.

Only one non-hypoxic lesion ( $T B R=1.37, H F=0$ ) at baseline, changed its hypoxic status during treatment. However, The $\mathrm{TBR}_{\max }$ increased only from 1.37 at baseline to 1.43 during treatment resulting in a small HF and HV during treatment of $0.4 \%$ and $0.2 \mathrm{~cm}^{3}$, respectively. 
Table 8.2: ${ }^{18}$ F]HX4 PET/CT parameters at baseline and during therapy. Shown are the mean, standard deviation, range and the percentage difference. Shown are the baseline hypoxic lesions $\left(G T V_{\text {prim }}\right.$ and $\left.G T V_{\text {In }}\right)$, with an $\left.{ }^{18} \mathrm{~F}\right] H X 4$ PET/CT scan at baseline and during treatment (total lesions $N=17$ ). The provided significance is based on the Wilcoxon signed rank test.

\begin{tabular}{lllll}
\hline & Baseline & During treatment & Difference [\%] & $\begin{array}{l}\text { Significance } \\
\text { (P-value) }\end{array}$ \\
\hline SUV $_{\text {mean }}$ & $0.9 \pm 0.2$ & $0.8 \pm 0.2$ & $-13 \pm 19$ & 0.02 \\
& $(0.7-1.3)$ & $(0.5-1.2)$ & & \\
SUV $_{\text {max }}$ & $1.5 \pm 0.4$ & $1.1 \pm 0.3$ & $-25 \pm 18$ & 0.001 \\
TMR $_{\text {max }}$ & $(0.9-2.1)$ & $(0.7-1.9)$ & & \\
& $1.9 \pm 0.4$ & $1.4 \pm 0.2$ & $-27 \pm 11$ & $<0.001$ \\
Hypoxic fraction $[\%]$ & $(1.4-2.8)$ & $(1.0-2.1)$ & & $<0.001$ \\
Hypoxic volume $\left[\mathrm{cm}^{3}\right]$ & $22 \pm 20$ & $4 \pm 10$ & $-93 \pm 15$ & \\
& $(3-71)$ & $(0-40)$ & & $<0.001$ \\
\hline
\end{tabular}

\section{Spatial stability of $\left[{ }^{18} \mathrm{~F}\right] \mathrm{HX} 4$ PET uptake}

To perform an analysis of the spatial $\left[{ }^{18} \mathrm{~F}\right] \mathrm{HX} 4$ uptake, a significant tracer uptake in both $\left[{ }^{18} \mathrm{~F}\right] \mathrm{HX} 4 \mathrm{PET} / \mathrm{CT}$ scans was necessary. Only 2 lesions (both $\mathrm{GTV}_{\mathrm{In}}$ ) had a HV larger than 1 $\mathrm{cm}^{3}$ during treatment $\left(2.9 \mathrm{~cm}^{3}\right.$ and $\left.10.1 \mathrm{~cm}^{3}\right)$. These $G_{T V}$ In were selected for the voxelwise analysis of the $\left[{ }^{18} \mathrm{~F}\right] \mathrm{HX} 4$ uptake within the GTV, resulting in a Pearson's correlation coefficient of 0.63 and 0.85 , respectively. The HV during treatment largely overlapped within the HV at baseline (98\% and $100 \%$, respectively; Figure 8.3 ).

\section{Blood biomarkers}

At baseline ( $\mathrm{N}=20)$ we measured an average concentration of $57 \pm 26 \mathrm{ng} / \mathrm{ml}$ osteopontin, $190 \pm 120 \mathrm{pg} / \mathrm{ml} \mathrm{CAIX}$ and $85 \pm 67 \mathrm{pg} / \mathrm{ml}$ VEGF. There was no inter-correlation between the different plasma parameters. During (chemo)radiotherapy $(\mathrm{N}=17)$, a non-significant decrease in CAIX $(173 \pm 97 \mathrm{pg} / \mathrm{ml} ; \mathrm{P}=0.45)$ and VEGF $(75 \pm 67 \mathrm{pg} / \mathrm{ml} ; \mathrm{P}=0.74)$ was observed, whereas the increase in osteopontin was significant $(65 \pm 31 \mathrm{pg} / \mathrm{ml} ; \mathrm{P}=0.04$; Figure S8.1).

\section{Relationship blood biomarkers and $\left[{ }^{18} \mathrm{~F}\right] \mathrm{HX} 4$ PET}

At baseline none of the blood biomarkers (CAIX, VEGF, osteopontin) showed a correlation with any of the $\left[{ }^{18} \mathrm{~F}\right] \mathrm{HX} 4$ PET image parameters. During treatment, only the osteopontin concentration was weakly correlated with the $\mathrm{SUV}_{\text {mean }}$ on the $\left[{ }^{18} \mathrm{~F}\right] \mathrm{HX} 4 \mathrm{PET}\left(\mathrm{R}_{\mathrm{s}}=0.52\right.$, $\mathrm{P}=0.03$; Figure S8.1). 


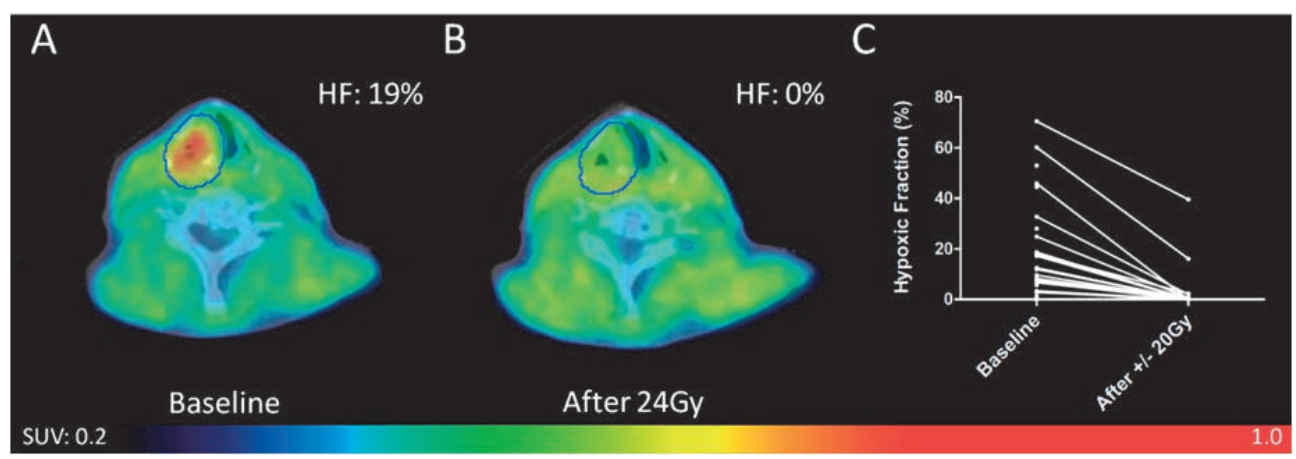

Figure 8.2: $\left[^{18} \mathrm{~F}\right] \mathrm{HX} 4$ PET/CT scan of a patient with a T3N2bMx squamous cell carcinoma of the hypopharynx treated with cisplatin chemo-radiation. A) Scan with hypoxic primary tumour at baseline, B) decreased level of hypoxia during treatment and C) Calculated hypoxic fraction (HF) of all primary tumours and lymph nodes before and during treatment, significant decrease $(p<0.001)$.
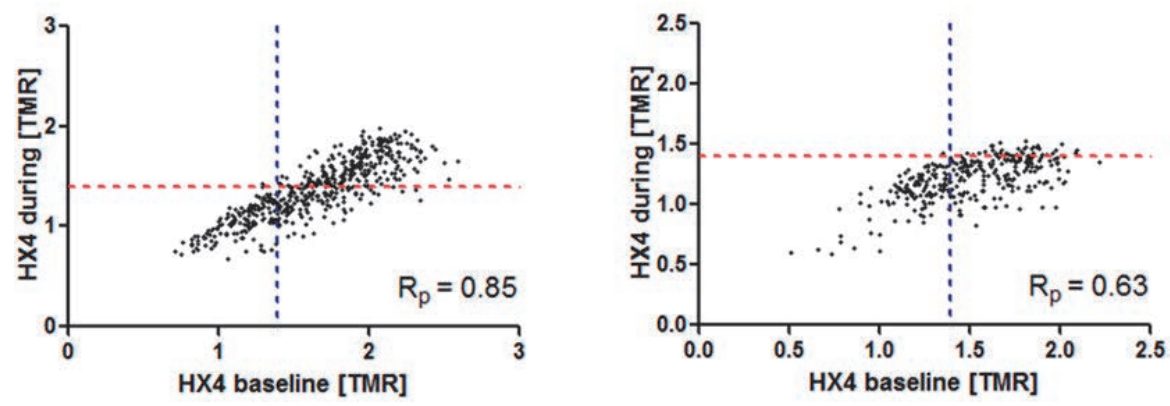

Figure 8.3: Spatial reproducibility of the $\left[^{18} \mathrm{~F}\right] H X 4$ PET uptake in two patients with persistent hypoxia during treatment (left: patient with CT2N2aMO hypopharynx cancer, right: patient with cT2N2bMO oropharynx cancer. The PET-CT scans during treatment were in both patients performed after $18 \mathrm{~Gy})$. Visualised is the voxel-wise correlation of the $\left[{ }^{18} \mathrm{~F}\right] \mathrm{HX} 4$-uptake within the GTV.

\section{DISCUSSION}

In this study we evaluated tumour hypoxia with $\left[{ }^{18} \mathrm{~F}\right] \mathrm{HX} 4$ PET in patients with HNSCC before the start of (chemo)radiotherapy and during treatment, with the aim to monitor treatment response and evaluate the spatial variability of the $\left[{ }^{18} \mathrm{~F}\right] \mathrm{HX} 4$ uptake. In addition, the concentration of blood hypoxia markers (osteopontin, CAIX and VEGF) was evaluated at baseline and during treatment. Last, the interdependence between hypoxia PET imaging and hypoxia-related blood biomarkers was investigated.

Before the start of (chemo)radiotherapy, we observed tumour hypoxia in the majority of primary HNSCC and metastatic lymph nodes. However, in most of these lesions hypoxia disappeared during the course of treatment, regardless of the chosen treatment; radiotherapy alone or in combination with cisplatin or cetuximab. The decrease of hypoxia during treatment has been described in several studies $(3,19-21)$. Lee et al. (19) showed with $\left[{ }^{18}\right.$ F]FMISO PET imaging a decrease in detectable tumour hypoxia from $90 \%(18 / 20$ patients) at baseline to $11 \%(2 / 18)$ after 4 weeks of chemoradiotherapy. In addition, 
Servagi-Vernat et al. (20) observed a decrease of the $\left[{ }^{18}\right.$ F]FAZA PET uptake (SUV $\max$ and HF) in all patients during chemoradiotherapy, which was recently confirmed by Bollineni et al.(21). From our current results and the previous literature we can conclude that in patients with HNSCC, hypoxia decreases during treatment, indicating tumour reoxygenation. Nevertheless, also a decrease in cell number, changes in vasculature or perfusion could contribute to the altered hypoxia PET uptake.

Additionally, our results show that the patients with a high uptake at baseline have the highest chance of persistent hypoxia during treatment. These patients may benefit most from anti-hypoxia therapy during the entire course of treatment. For the other cases, the addition of anti-hypoxia therapy will probably have the largest therapeutic effect when given prior to, or during the first weeks of treatment. Since after this period, the amount of tumour hypoxia is low. $\left[{ }^{18} \mathrm{~F}\right] \mathrm{HX} 4$ PET imaging has already shown its potential to monitor the response to the anti-hypoxia treatment with $\mathrm{TH}-302$ (22). The ability to monitor the response to anti-hypoxia treatment with non-invasive imaging provides the potential to adapt the anti-hypoxia treatment based on the (changing) lesion characteristics.

Another frequently discussed method to target resistant tumour volumes is by giving a radiation boost. Previous studies have shown that it is technically feasible to provide a radiotherapy boost to hypoxic or metabolically active tumour subvolumes, defined on PET $(4,23-25)$. For this purpose, information on the spatial repeatability of the hypoxia PET uptake and its stability during treatment is essential. In our current data-set only two lesions showed a significant amount of tumour hypoxia during treatment, with a spatially stable localization in comparison to the baseline scan. Where Bittner et al. (26) reported a geographically stable localization of the $\left[{ }^{18} \mathrm{~F}\right] \mathrm{FMISO}$ PET uptake during chemoradiotherapy, Nehmeh et al.(27), Lin et al.(28) and Servagi-Vernat et al.(20) reported changes in the distribution of hypoxia during treatment, and therefore adaptive radiotherapy based on serial hypoxia PET imaging was recommended.

Serial hypoxia PET imaging during treatment may provide additional information for response prediction. In preclinical setting, micro-environmental parameters (hypoxia, perfusion) during treatment had a better potential to predict outcome after radiotherapy (29). This finding was confirmed in a clinical study by Zips et al. (3) in 25 patients with HNSCC. The authors found a significant correlation between $\left[{ }^{18} \mathrm{~F}\right] \mathrm{FMISO}-\mathrm{PET}$ imaging before and during chemoradiotherapy and local progression free survival, and tumour hypoxia during treatment was of higher prognostic relevance. In our study, accrual is ongoing and the assessment of hypoxia in relationship to outcome will be performed as data have matured.

We hypothesized that blood biomarkers could also be used to monitor treatment response, because previous studies have showed that blood biomarkers have the potential to predict response to treatment (30). For example, a high concentration of plasma osteopontin was related to a higher amount of locoregional tumour failure in patients with HNSCC (14). The blood biomarkers CAIX and VEGF, showed no significant changes during treatment and the osteopontin concentration was increased, while hypoxia PET imaging showed a clear reduction of the uptake in all patients. These results might be explained by the patient cohort. When comparing the plasma osteopontin levels of the patients in our 
current trial to the levels reported in the large randomized trial from Overgaard et al.(14), we observed that all our patients should be assigned to the low or intermediate concentrations of plasma osteopontin. Also in comparison to a study in 295 patients with advanced rectal cancer, our observed levels of osteopontin and CAIX were lower than their reported average values (30). In addition, plasma osteopontin is not only influenced by hypoxia, it is also known to play a role in the immune regulation and stress response (31). An elevated level of osteopontin was for example observed in patients with systemic inflammatory response syndrome or sepsis (32). The given anti-cancer treatment might have induced an immune/stress response causing an elevated level of hypoxia. Nevertheless, in the current population the blood biomarkers osteopontin, CAIX and VEGF were not suitable to measure a hypoxia related treatment response, which was measurable using $\left[{ }^{18} \mathrm{~F}\right] \mathrm{HX} 4-\mathrm{PET}$.

The correlation between the blood hypoxia markers and the $\left[{ }^{18} \mathrm{~F}\right] \mathrm{HX} 4$ PET uptake was absent to weakly present. This was in agreement with the results previously published by several groups comparing hypoxia PET imaging to tissue or blood markers. Vercellino et al.(33) observed no correlation between the uptake of the hypoxia tracer $\left[{ }^{18}\right.$ F]FETNIM and the plasma osteopontin concentration in 16 patients with cervical carcinoma. In addition, Gronroos et al.(34) found no correlation between hypoxia PET imaging using FETNIM and several tissue biomarkers in 15 patients with HNSCC. While in patients with newly diagnosed glioma, the preoperative $\left[{ }^{18} \mathrm{~F}\right] \mathrm{FMISO}$ PET uptake was significantly, but weakly correlated to the expression of VEGF in the tumour(35). Although the current patient population is small, the results suggest that the tested blood biomarkers are not able to replace hypoxia PET imaging, or to pre-select patients for hypoxia PET imaging.

To conclude, hypoxia PET imaging with $\left[{ }^{18} \mathrm{~F}\right] \mathrm{HX} 4$ is able to detect tumour hypoxia in patients with HNSCC, in addition it can monitor a decrease of tumour hypoxia during treatment. In patients with persistent tumour hypoxia, a stable localization of the hypoxic volume was observed. This provides potential for radiotherapy dose escalation to the hypoxic volumes. The blood parameters osteopontin, CAIX and VEGF were not able to detect a decrease in hypoxia during treatment. In addition, there was no correlation between the blood plasma parameters CAIX and VEGF and hypoxia PET imaging, while only a weak correlation was observed between $\left[{ }^{18} \mathrm{~F}\right] \mathrm{HX} 4 \mathrm{PET}$ imaging and the osteopontin concentration during treatment. Based on the current data, we conclude that hypoxia PET imaging is the superior method to evaluate tumour hypoxia before and during treatment and cannot be replaced with the evaluated blood biomarkers.

\section{ACKNOWLEDGEMENTS}

The authors like to thank the patients who agreed to participate and the PET-CT group, datamanagement (Anita Botterweck) and trial-poli (Claudia Offermann and John Paulissen) of Maastro Clinic for their contribution to the data acquisition. Authors acknowledge financial support from the QuIC-ConCePT project, which is partly funded by EFPI A companies and the Innovative Medicine Initiative Joint Undertaking (IMI JU) under Grant Agreement No. 115151. Authors also acknowledge financial support from EU 7th framework program 
(EURECA, ARTFORCE - $\mathrm{n}^{\circ}$ 257144, REQUITE - $\mathrm{n}^{\circ}$ 601826), Kankeronderzoekfonds Limburg from the Health Foundation Limburg and the Dutch Cancer Society (KWF MAC 2011-4970).

\section{SUPPLEMENTARY DATA}

A

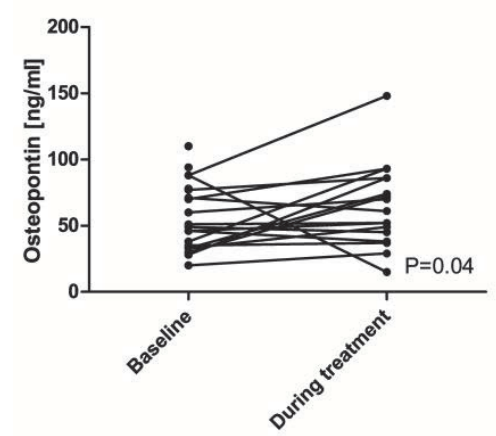

B

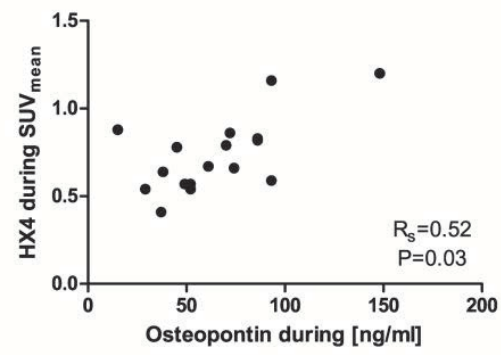

Figure S8.1: A) Observed increase in plasma osteopontin during treatment and B) significant correlation between the blood biomarker osteopontin, measured during treatment and SUV $V_{\text {mean }}$ on the $\left[{ }^{18} \mathrm{~F}\right] \mathrm{HX} 4 \mathrm{PET} / \mathrm{CT}$.

Table S8.1: $\left[{ }^{18} \mathrm{~F}\right] \mathrm{HX} 4$ PET/CT image derived parameters at baseline and during therapy; data was split based on the anti-cancer treatment. Shown are the mean, standard deviation, range and the percentage difference of the baseline hypoxic lesions (GTV prim and GTV ), with an $\left.{ }^{18}{ }^{18} \mathrm{~F}\right] \mathrm{HX} 4 \mathrm{PET} / \mathrm{CT}$ scan at baseline and during treatment (total lesions: $N=17)$.

\begin{tabular}{|c|c|c|c|}
\hline & Baseline & During treatment & Difference [\%] \\
\hline \multicolumn{4}{|c|}{ Cisplatin chemo-radiotherapy $(\mathrm{N}=10)$} \\
\hline $\mathrm{TMR}_{\max }$ & $\begin{array}{l}2.0 \pm 0.5 \\
(1.4-2.8)\end{array}$ & $\begin{array}{l}1.4 \pm 0.3 \\
(1.0-2.1)\end{array}$ & $-29 \pm 12$ \\
\hline Hypoxic fraction [\%] & $\begin{array}{l}28 \pm 24 \\
(3-71)\end{array}$ & $\begin{array}{l}6 \pm 13 \\
(0-40)\end{array}$ & $-91 \pm 19$ \\
\hline Hypoxic volume $\left[\mathrm{cm}^{3}\right]$ & $\begin{array}{l}27.8 \pm 23.6 \\
(2.9-70.5)\end{array}$ & $\begin{array}{l}1.4 \pm 3.2 \\
(0.0-10.1)\end{array}$ & $-91 \pm 19$ \\
\hline \multicolumn{4}{|l|}{ Radiotherapy ( $\mathrm{N}=4$ ) } \\
\hline $\mathrm{TMR}_{\max }$ & $\begin{array}{l}1.9 \pm 0.3 \\
(1.7-2.3)\end{array}$ & $\begin{array}{l}1.4 \pm 0.1 \\
(1.3-1.5)\end{array}$ & $-23 \pm 13$ \\
\hline Hypoxic fraction [\%] & $\begin{array}{l}14 \pm 5 \\
(7-18)\end{array}$ & $\begin{array}{l}1 \pm 1 \\
(0-2)\end{array}$ & $-94 \pm 6$ \\
\hline Hypoxic volume $\left[\mathrm{cm}^{3}\right]$ & $\begin{array}{l}3.5 \pm 1.9 \\
(0.7-5.1)\end{array}$ & $\begin{array}{l}0.2 \pm 0.3 \\
(0.0-0.6)\end{array}$ & $-94 \pm 6$ \\
\hline \multicolumn{4}{|c|}{ Cetuximab-radiotherapy $(\mathrm{N}=2)$} \\
\hline $\mathrm{TMR}_{\max }$ & $\begin{array}{l}1.7 \pm 0.4 \\
(1.5-2.1)\end{array}$ & $\begin{array}{l}1.3 \pm 0.2 \\
(1.2-1.5)\end{array}$ & $-25 \pm 5$ \\
\hline Hypoxic fraction [\%] & $\begin{array}{l}12 \pm 12 \\
(3-25)\end{array}$ & $\begin{array}{l}0.2 \pm 0.3 \\
(0.0-0.5)\end{array}$ & $-99 \pm 1$ \\
\hline Hypoxic volume $\left[\mathrm{cm}^{3}\right]$ & $\begin{array}{l}1.1 \pm 1.1 \\
(0.4-2.4)\end{array}$ & $\begin{array}{l}0.0 \pm 0.0 \\
(0.0-0.1)\end{array}$ & $-99 \pm 1$ \\
\hline
\end{tabular}




\section{REFERENCES}

1. Nordsmark M, Bentzen SM, Rudat V, Brizel D, Lartigau E, Stadler P, et al. Prognostic value of tumor oxygenation in 397 head and neck tumors after primary radiation therapy. An international multi-center study. Radiother Oncol. 2005;77:18-24.

2. Horsman MR, Mortensen LS, Petersen JB, Busk M, Overgaard J. Imaging hypoxia to improve radiotherapy outcome. Nat Rev Clin Oncol. 2012;9:674-87.

3. Zips D, Zophel K, Abolmaali N, Perrin R, Abramyuk A, Haase R, et al. Exploratory prospective trial of hypoxia-specific $\mathrm{PET}$ imaging during radiochemotherapy in patients with locally advanced head-and-neck cancer. Radiother Oncol. 2012;105:21-8.

4. Hendrickson K, Phillips M, Smith W, Peterson L, Krohn K, Rajendran J. Hypoxia imaging with [F-18] FMISO-PET in head and neck cancer: potential for guiding intensity modulated radiation therapy in overcoming hypoxia-induced treatment resistance. Radiother Oncol. 2011;101:369-75.

5. van Elmpt $W$, Ollers $M$, Dingemans $A M$, Lambin $P$, De Ruysscher $D$. Response assessment using 18F-FDG PET early in the course of radiotherapy correlates with survival in advanced-stage non-small cell lung cancer. J Nucl Med. 2012;53:1514-20.

6. Overgaard J. Hypoxic modification of radiotherapy in squamous cell carcinoma of the head and neck--a systematic review and meta-analysis. Radiother Oncol. 2011;100:22-32.

7. Kaanders JH, Wijffels KI, Marres HA, Ljungkvist AS, Pop LA, van den Hoogen FJ, et al. Pimonidazole binding and tumor vascularity predict for treatment outcome in head and neck cancer. Cancer Res. 2002;62:7066-74.

8. Janssens GO, Rademakers SE, Terhaard CH, Doornaert PA, Bijl HP, van den Ende P, et al. Accelerated radiotherapy with carbogen and nicotinamide for laryngeal cancer: results of a phase III randomized trial. J Clin Oncol. 2012;30:1777-83.

9. Dubois $L$, Lieuwes NG, Janssen MH, Peeters WJ, Windhorst AD, Walsh JC, et al. Preclinical evaluation and validation of [18F]HX4, a promising hypoxia marker for PET imaging. Proc Natl Acad Sci U S A. 2011;108:14620-5.

10. Peeters SG, Zegers CM, Lieuwes NG, van Elmpt W, Eriksson J, van Dongen GA, et al. A Comparative Study of the Hypoxia PET Tracers [(18)F]HX4, [(18)F]FAZA, and [(18)F]FMISO in a Preclinical Tumor Model. Int J Radiat Oncol Biol Phys. 2015;91:351-9.

11. Zegers CM, van Elmpt W, Reymen B, Even AJ, Troost EG, Ollers MC, et al. In vivo quantification of hypoxic and metabolic status of NSCLC tumors using [18F]HX4 and [18F]FDG-PET/CT imaging. Clin Cancer Res. 2014;20:6389-97.

12. Zegers $C M$, van Elmpt W, Wierts $R$, Reymen B, Sharifi H, Ollers MC, et al. Hypoxia imaging with $[(1)(8) F] H X 4$ PET in NSCLC patients: defining optimal imaging parameters. Radiother Oncol. 2013;109:58-64.

13. Nordsmark M, Eriksen JG, Gebski V, Alsner J, Horsman MR, Overgaard J. Differential risk assessments from five hypoxia specific assays: The basis for biologically adapted individualized radiotherapy in advanced head and neck cancer patients. Radiother Oncol. 2007;83:389-97.

14. Overgaard J, Eriksen JG, Nordsmark M, Alsner J, Horsman MR. Plasma osteopontin, hypoxia, and response to the hypoxia sensitiser nimorazole in radiotherapy of head 
and neck cancer: results from the DAHANCA 5 randomised double-blind placebocontrolled trial. Lancet Oncol. 2005;6:757-64.

15. Ilie M, Mazure NM, Hofman V, Ammadi RE, Ortholan C, Bonnetaud C, et al. High levels of carbonic anhydrase IX in tumour tissue and plasma are biomarkers of poor prognostic in patients with non-small cell lung cancer. Br J Cancer. 2010;102:1627-35.

16. van Loon J, Janssen MH, Ollers $M$, Aerts HJ, Dubois L, Hochstenbag M, et al. PET imaging of hypoxia using [18F]HX4: a phase I trial. Eur J Nucl Med Mol Imaging. 2010;37:1663-8.

17. Doss $M$, Zhang JJ, Belanger MJ, Stubbs JB, Hostetler ED, Alpaugh K, et al. Biodistribution and radiation dosimetry of the hypoxia marker 18F-HX4 in monkeys and humans determined by using whole-body PET/CT. Nucl Med Commun. 2010;31:1016-24.

18. Chen L, Zhang Z, Kolb HC, Walsh JC, Zhang J, Guan Y. (1)(8)F-HX4 hypoxia imaging with PET/CT in head and neck cancer: a comparison with (1)(8)F-FMISO. Nucl Med Commun. 2012;33:1096-102.

19. Lee N, Nehmeh S, Schoder H, Fury M, Chan K, Ling CC, et al. Prospective trial incorporating pre-/mid-treatment [18F]-misonidazole positron emission tomography for head-and-neck cancer patients undergoing concurrent chemoradiotherapy. Int J Radiat Oncol Biol Phys. 2009;75:101-8.

20. Servagi-Vernat S, Differding S, Hanin FX, Labar D, Bol A, Lee JA, et al. A prospective clinical study of (1)(8)F-FAZA PET-CT hypoxia imaging in head and neck squamous cell carcinoma before and during radiation therapy. Eur J Nucl Med Mol Imaging. 2014;41:1544-52.

21. Bollineni VR, Koole MJ, Pruim J, Brouwer CL, Wiegman EM, Groen HJ, et al. Dynamics of tumor hypoxia assessed by 18F-FAZA PET/CT in head and neck and lung cancer patients during chemoradiation: possible implications for radiotherapy treatment planning strategies. Radiother Oncol. 2014;113:198-203.

22. Peeters SG, Zegers CM, Biemans R, Lieuwes NG, van Stiphout RG, Yaromina A, et al. $\mathrm{TH}-302$ in combination with radiotherapy enhances the therapeutic outcome and is associated with pretreatment [18F]HX4 hypoxia PET imaging. Clin Cancer Res. 2015.

23. Servagi-Vernat S, Differding S, Sterpin E, Hanin FX, Labar D, Bol A, et al. Hypoxiaguided adaptive radiation dose escalation in head and neck carcinoma: A planning study. Acta Oncol. 2015:1-9.

24. Choi W, Lee SW, Park SH, Ryu JS, Oh SJ, Im KC, et al. Planning study for available dose of hypoxic tumor volume using fluorine-18-labeled fluoromisonidazole positron emission tomography for treatment of the head and neck cancer. Radiother Oncol. 2010;97:176-82.

25. Berwouts D, Olteanu LA, Duprez F, Vercauteren T, De Gersem W, De Neve W, et al. Three-phase adaptive dose-painting-by-numbers for head-and-neck cancer: initial results of the phase I clinical trial. Radiother Oncol. 2013;107:310-6.

26. Bittner MI, Wiedenmann N, Bucher S, Hentschel M, Mix M, Weber WA, et al. Exploratory geographical analysis of hypoxic subvolumes using 18F-MISO-PET imaging in patients with head and neck cancer in the course of primary chemoradiotherapy. Radiother Oncol. 2013;108:511-6. 
27. Nehmeh SA, Lee NY, Schroder H, Squire O, Zanzonico PB, Erdi YE, et al. Reproducibility of intratumor distribution of (18)F-fluoromisonidazole in head and neck cancer. Int J Radiat Oncol Biol Phys. 2008;70:235-42.

28. Lin Z, Mechalakos J, Nehmeh S, Schoder H, Lee N, Humm J, et al. The influence of changes in tumor hypoxia on dose-painting treatment plans based on 18F-FMISO positron emission tomography. Int J Radiat Oncol Biol Phys. 2008;70:1219-28.

29. Yaromina A, Kroeber T, Meinzer A, Boeke S, Thames H, Baumann M, et al. Exploratory study of the prognostic value of microenvironmental parameters during fractionated irradiation in human squamous cell carcinoma xenografts. Int J Radiat Oncol Biol Phys. 2011;80:1205-13.

30. Buijsen J, van Stiphout RG, Menheere PP, Lammering G, Lambin P. Blood biomarkers are helpful in the prediction of response to chemoradiation in rectal cancer: a prospective, hypothesis driven study on patients with locally advanced rectal cancer. Radiother Oncol. 2014;111:237-42.

31. Wang KX, Denhardt DT. Osteopontin: role in immune regulation and stress responses. Cytokine Growth Factor Rev. 2008;19:333-45.

32. Vaschetto R, Nicola S, Olivieri C, Boggio E, Piccolella F, Mesturini R, et al. Serum levels of osteopontin are increased in SIRS and sepsis. Intensive Care Med. 2008;34:217684.

33. Vercellino L, Groheux D, Thoury A, Delord M, Schlageter MH, Delpech $\mathrm{Y}$, et al. Hypoxia imaging of uterine cervix carcinoma with (18)F-FETNIM PET/CT. Clin Nucl Med. 2012;37:1065-8.

34. Gronroos TJ, Lehtio K, Soderstrom KO, Kronqvist P, Laine J, Eskola O, et al. Hypoxia, blood flow and metabolism in squamous-cell carcinoma of the head and neck: correlations between multiple immunohistochemical parameters and PET. BMC Cancer. 2014;14:876.

35. Kawai N, Lin W, Cao WD, Ogawa D, Miyake K, Haba R, et al. Correlation between (1)(8)F-fluoromisonidazole PET and expression of HIF-1alpha and VEGF in newly diagnosed and recurrent malignant gliomas. Eur J Nucl Med Mol Imaging. 2014;41:1870-8. 



\section{CHAPTER}

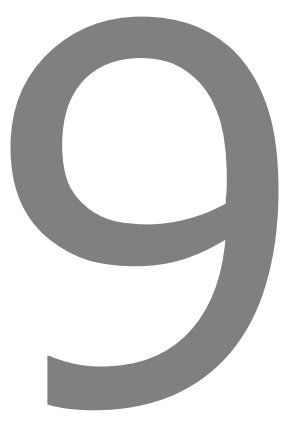

$\mathrm{TH}-302$ in combination with radiotherapy enhances the therapeutic outcome and is associated with pretreatment $\left[{ }^{18} \mathrm{~F}\right] \mathrm{HX} 4$ hypoxia PET imaging

Sarah G.J.A. Peeters, Catharina M.L. Zegers, Rianne Biemans, Natasja G. Lieuwes, Ruud G.P.M. van Stiphout, Ala Yaromina, Jessica D. Sun, Charles P. Hart, Albert D. Windhorst, Wouter van Elmpt, Ludwig Dubois*, Philippe Lambin* 


\section{ABSTRACT}

Purpose: Conventional anticancer treatments are often impaired by the presence of hypoxia. TH-302 selectively targets hypoxic tumor regions where it is converted into a cytotoxic agent. This study assessed the efficacy of the combination treatment of TH-302 and radiotherapy in two preclinical tumor models. The effect of oxygen modification on the combination treatment was evaluated and the effect of $\mathrm{TH}-302$ on the hypoxic fraction (HF) was monitored using $\left[{ }^{18} \mathrm{~F}\right] \mathrm{HX} 4-\mathrm{PET}$ imaging and pimonidazole IHC stainings.

Experimental Design: Rhabdomyosarcoma R1 and H460 NSCLC tumor-bearing animals were treated with $\mathrm{TH}-302$ and radiotherapy ( $8 \mathrm{~Gy}$, single dose). The tumor oxygenation status was altered by exposing animals to carbogen (95\% oxygen) and nicotinamide, $21 \%$ or $7 \%$ oxygen breathing during the course of the treatment. Tumor growth and treatment toxicity was monitored until the tumor reached 4 times its start volume (T4xSV).

Results: Both tumor models showed a growth delay after TH-302 treatment which further increased when combined with radiotherapy (enhancement ratio rhabdomyosarcoma 1.23; H460 1.49). TH-302 decreases the HF in both models, consistent with its hypoxiatargeting mechanism of action. Treatment efficacy was dependent on tumor oxygenation; increasing the tumor oxygen status abolished the effect of $\mathrm{TH}-302$, whereas enhancing the HF enlarged TH-302's therapeutic effect. An association was observed in rhabdomyosarcoma tumors between the pretreatment HF as measured by $\left[{ }^{18} \mathrm{~F}\right] \mathrm{HX} 4-\mathrm{PET}$ imaging and the T4xSV.

Conclusions: The combination of $\mathrm{TH}-302$ and radiotherapy is promising and warrants clinical testing, preferably guided by the companion biomarker $\left[{ }^{18} \mathrm{~F}\right] \mathrm{HX} 4$ hypoxia PET imaging for patient selection. 


\section{INTRODUCTION}

Hypoxia is a common feature of solid tumors and is known to negatively influence treatment outcome $(1,2)$. Because of the disorganized vessel formation and consequently low oxygen concentrations, conventional chemotherapies and radiotherapies are less effective. To overcome hypoxia-induced treatment resistance, drugs have been developed that specifically target hypoxic tumor regions. These so-called 'hypoxia activated prodrugs' (HAP) are non-toxic under normal oxygen concentrations but are activated in environments with low oxygen concentrations. TH-302 is a second generation HAP of which the activation mechanism is based on the reduction of its 2-nitroimidazole moiety. Only in the presence of certain reductases under low oxygen concentrations is the toxic effector bromo-isophosphoramide mustard (Br-IPM) selectively released and able to crosslink DNA leading to cell death.

Preclinical studies have assessed the therapeutic effect of TH-302 alone or in combination with conventional chemotherapies in multiple xenograft models. TH-302 monotherapy led to reduced tumor growth in many of the xenograft models profiled, and was dependent on the hypoxic fraction (HF; ref 3). Combining TH-302 with several clinically used chemotherapeutics offers advantage over single-agent treatment, although the treatment schedule and order of administration is of importance (4). A phase 1 study demonstrated the safety of TH-302 monotherapy in patients with solid malignancies (5). Other clinical phase I and II trials successfully combined the standard treatment doxorubicin with intravenous administration of $\mathrm{TH}-302$ in patients with advanced soft tissue sarcoma $(6,7)$ and standard treatment gemcitabine with $\mathrm{TH}-302$ in patients with advanced pancreatic cancer (8).

However, there are no published studies to assess the combination therapy of TH-302 and radiotherapy. Radiotherapy is one of the conventional treatment options applied to multiple cancer types and tumor hypoxia is a known radioresistance factor. The combination of TH-302 and radiotherapy is hypothesized to be complementary; where $\mathrm{TH}-302$ specifically targets hypoxic cells, radiotherapy has the highest therapeutic gain in the welloxygenated cells.

PET imaging is a non-invasive method characterizing the tumor oxygenation status in a three-dimensional manner. Several PET tracers have been developed that specifically visualize hypoxic regions. One of those tracers, based on the same 2-nitroimidazole hypoxia sensing mechanism as $\mathrm{TH}-302$, is $\left[{ }^{18} \mathrm{~F}\right] \mathrm{HX} 4$. Preclinical and clinical trials have shown that $\left[{ }^{18} \mathrm{~F}\right] \mathrm{HX} 4$ is a reliable tool for the noninvasive detection of hypoxic tumor regions (9-11). Because TH-302, like tirapazamine, is expected to have only a therapeutic effect when hypoxic regions are present in the tumor (12), $\left[{ }^{18} \mathrm{~F}\right] \mathrm{HX} 4$ PET imaging may function as a useful predictive biomarker.

In the current study we investigated the treatment effect of TH-302 in combination with radiotherapy in two preclinical tumor models. We hypothesize that this combination therapy will enhance the therapeutic effect. Furthermore we investigate the causal rela- 
tionship between TH-302 efficacy and the modified tumor oxygenation status which was assessed before and after treatment using $\left[{ }^{18} \mathrm{~F}\right] \mathrm{HX} 4$ hypoxia PET imaging and only after treatment with pimonidazole staining. We hypothesize that the pretreatment hypoxia $\left[{ }^{18} \mathrm{~F}\right] \mathrm{HX} 4 \mathrm{PET}$ imaging will correlate with the treatment outcome.

\section{MATERIALS AND METHODS}

\section{Animal, tumor models and treatment schedules}

All animal experimental procedures were approved by the Animal Ethical Committee of Maastricht University and were in accordance with the Helsinki Declaration of 1975 as revised in 2000. All animals were monitored at least 3 times a week and their tumor volume was calculated using the formula: $A \times B \times C \times \pi / 6$ in which $A, B$ and $C$ are the three orthogonal diameters of the tumor as measured using a Vernier caliper, each corrected for the thickness of the skin. Animals were randomized into the different treatment groups (Figure S9.1A) and were monitored until four times start volume (T4xSV) was reached. For this calculation the start volume of the first day of TH-302 treatment was used and fitting of the data was based on the regrowth phase. TH-302 was supplied by Threshold Pharmaceuticals and dissolved in $0.9 \% \mathrm{NaCl}$ to a concentration of $5 \mathrm{mg} / \mathrm{mL}$.

\section{Experimental models}

Syngeneic rhabdomyosarcoma R1 tumors $\left(1 \mathrm{~mm}^{3}\right)$ were implanted subcutaneously in the lateral flank of adult WAG/Rij rats. Experiments were started upon a mean tumor volume of $4.2 \mathrm{~cm}^{3}$ (range, 2.0-8.1) to ensure a stable HF. Treatment was administered on 4 consecutive days (Figure S9.1A) and consisted of an intraperitoneal injection (i.p.; QDx4) with either $\mathrm{NaCl}$ or $\mathrm{TH}-302(25,50$ or $75 \mathrm{mg} / \mathrm{kg})$. Before the start of treatment a PET scan was made using $\left[{ }^{18} \mathrm{~F}\right] \mathrm{HX} 4$. Radiotherapy (Varian Truebeam linear accelerator; $15 \mathrm{MeV}$ electrons) was applied in a single dose of $0,4,8$ or 12 Gy on day 3 of the treatment, 3 hours after $\mathrm{NaCl}$ or $\mathrm{TH}-302$ injection, 1 hour after oxygen modification. During both PET imaging and radiotherapy rats were anaesthetized using a mixture of ketamine/xylazine (i.p; 66.7 and $6.7 \mathrm{mg} / \mathrm{kg}$, respectively). During the 5 days of treatment (1 day PET imaging, 4 days of injections with $\mathrm{TH}-302$ or vehicle), animals were exposed to modified oxygen concentrations for 4 hours per day in order to alter the HF of the tumor. The combination oxygen modification of nicotinamide (i.p. $500 \mathrm{mg} / \mathrm{kg}$ ) and carbogen ( $95 \%$ oxygen, $5 \% \mathrm{CO}_{2} ; 5 \mathrm{~L} / \mathrm{min}$ ) consisted of a nicotinamide injection and 30 minutes later the exposure to carbogen breathing for 3.5 hours. In the middle of the nicotinamide/carbogen treatment $\mathrm{NaCl} / \mathrm{TH}-$ 302 was administered. Reduced oxygen breathing (7\%, residual $\mathrm{N}_{2} ; 2.5 \mathrm{~L} / \mathrm{min}$ ) was given for 4 hours with the $\mathrm{NaCl} / \mathrm{TH}-302$ injection after the first two hours. The injection of the $\left[{ }^{18} \mathrm{~F}\right] \mathrm{HX} 4$ PET tracer [mean $18.8 \mathrm{MBq}$, range 7.1-25.1 MBq; lateral tail vein using an intravenous line (Venoflux $0.4 \mathrm{~mm} \mathrm{G27)} \mathrm{flushed} \mathrm{with} 10 \%$ heparine] was given 2 hours before the end of the oxygen modification. PET imaging was performed 3 hours after tracer injection, as previously assessed (13). $\left[{ }^{18} \mathrm{~F}\right] \mathrm{HX} 4$ PET scans could not be performed on all treated animals due to production and supply difficulties. For the histological control animals, PET imaging was also performed on day 4 of the treatment. 
H460 lung carcinoma cells were resuspended ( 1 × $10^{6}$ cells $/ \mathrm{mL}$; ATCC HTB-177) in Basement Membrane Matrix (Matrigel ${ }^{\mathrm{TM}} \mathrm{BD}$ Biosciences) and injected in the lateral flank of NU-Foxn1-nu (NU/NU) mice. Experiments were started upon animals reaching a mean tumor volume of $225 \mathrm{~mm}^{3}$ (range $89-273 \mathrm{~mm}^{3}$ ). Mice were treated with either $\mathrm{NaCl}$ or TH$302(50 \mathrm{mg} / \mathrm{kg}$ ) for 5 consecutive days (QDx5). Treatment was combined with radiotherapy in a single dose of 0 or 8 Gy on day 4 for which the animals were anaesthetized using a mixture of ketamine/xylazine (i.p; 66.7 and $6.7 \mathrm{mg} / \mathrm{kg}$ respectively). During the 5 days of treatment, animals were exposed to different oxygen concentrations; either a combination of nicotinamide (500 mg/kg i.p.) and carbogen breathing ( $95 \%$ oxygen, $5 \% \mathrm{CO}$ ), $21 \%$ oxygen breathing (pressured air) or $7 \%$ oxygen breathing (residual $\mathrm{N}_{2}$ ). Total treatment time for all oxygen breathing schedules was 2.5 hours with the $\mathrm{NaCl} / \mathrm{TH}-302$ injection given 1 hour after the start of the treatment. Nicotinamide was administered 30 minutes before carbogen breathing was started, which was then applied for another 2 hours. Radiotherapy was given within 1 hour after the oxygen treatment. Histological controls were administered with pimonidazole $(60 \mathrm{mg} / \mathrm{kg}$, i.p.; Hypoxyprobe kit, HP3-1000; Bioconnect) and Hoechst 33342 (15 mg/kg, i.v.; Sigma-Aldrich) 1 hour and 1 minute before sacrificing, respectively, followed by excision of the tumors which were snap frozen in liquid nitrogen and stored at -80 degrees Celsius until being processed.

\section{PET Image acquisition and analysis}

Tracer synthesis of $\left[{ }^{18} \mathrm{~F}\right] \mathrm{HX} 4$ was performed as described previously (14). $\left[{ }^{18} \mathrm{~F}\right] \mathrm{HX} 4$ positron emission tomography (PET) images were acquired and analyzed using a clinical PET/CT scanner (Siemens Biograph 40, Siemens Healthcare) as previously described (13). A volume of interest in the heart (sphere with a radius of $3 \mathrm{~mm}$ ) was defined as background region. The threshold to define the hypoxic fraction (HF) was set at 4.5 times the background uptake, because this method results in a HF which is in agreement with the pimonidazole staining- based results of a previous study on the rat rhabdomyosarcoma model (15).

\section{Immunohistochemical staining and analysis}

Frozen $\mathrm{H} 460$ xenograft tumors were sectioned $(5 \mu \mathrm{m})$ and stained for hypoxia (pimonidazole), blood vessels (CD31) and perfusion (Hoechst 33342). Sections were fixed using cold acetone, rehydrated in TBS with $0.2 \%$ Tween-20 (TBST) and preincubated with normal goat serum before exposing them to the primary antibody rabbit anti-pimonidazole (1:150; HP3-1000, Bio-connect) and rat anti-mouse CD31 (1:500; BD biosciences). After washing with TBST, incubation with the secondary antibody goat anti-rabbit Alexa594 (1:500) and goat anti-rat Alexa488 (1:750, both Invitrogen) was performed. Sections were mounted using fluorescent mounting medium (DakoCytomation) and scanned for pimonidazole, blood vessels and perfusion. After scanning, sections were stained for hematoxylin and eosin (H\&E).

Images were acquired using an Olympus BX51WI microscope equipped with a Hamamatsu EM-CCD C9100 digital camera, a motorized stage (Ludl Mac 2000) and a 10x objective. Micromanager 1.4 software was used for automated image acquisition (16). ImageJ version 1.49e (http://rsb.info.nih.gov/ij/) was used to stitch the images and perform 
quantitative analyses. All image recordings and analyses were performed by an investigator blinded to the subject coding. Viable tumor tissue was first delineated manually by excluding epidermis, stroma and necrotic tumor regions based on H\&E staining. The thresholds were set manually by two independent observers to discriminate signal from background. Finally the relative HF, microvessel density and perfusion were calculated by determining the positive fraction within the viable tumor area.

\section{Statistics}

GraphPad Prism software (version 5.01 for Windows) was used to perform statistical analyses. To determine the statistical significance of differences between two independent groups of variables we used an unpaired $t$ test, whereas for matched groups a paired $t$ test was applied. A two-way ANOVA was performed in R v3.0.1 to determine synergistic effects. $P$ values $<0.05$ were considered to be significant.

\section{RESULTS}

\section{Combination of TH-302 and radiotherapy}

The effect of TH-302 in combination with radiotherapy was assessed in two tumor models, a rhabdomyosarcoma rat syngeneic model and a $\mathrm{H} 460$ human non-small cell lung cancer (NSCLC) xenograft mouse model. The treatment dose of TH-302 for rhabdomyosarcoma was assessed in a 'tolerability' study, showing $25 \mathrm{mg} / \mathrm{kg}$ (QDx4) to be the most optimal dose without adverse effects (Figure S9.2). This dose was therefore selected for further experiments. The TH-302 treatment dose of $50 \mathrm{mg} / \mathrm{kg}$ (QDx5) for $\mathrm{H} 460$ was based on literature (3).

In both tumor models $\mathrm{TH}-302$ treatment showed an inhibition of the tumor growth which was further reduced when $\mathrm{TH}-302$ administration was combined with a single dose of radiotherapy ( $8 \mathrm{~Gy}$; Figure 9.1A). The time to reach 4 times start volume (T4xSV, Figure 9.1B) was significantly delayed upon $\mathrm{TH}-302$ monotherapy from $12.4 \pm 1.7$ (mean $\pm \mathrm{SD}$ ) to $20.4 \pm 3.5$ days for rhabdomyosarcoma $(P<0.001)$ and $7.1 \pm 2.4$ to $13.6 \pm 4.8$ days for $\mathrm{H} 460(\mathrm{P}=0.003)$. Compared to radiotherapy alone, T4XSV for the combination treatment was delayed from $24.9 \pm 3.0$ to $30.8 \pm 5.9$ for rhabdomyosarcoma $(P=0.026)$ and from $16.9 \pm 7.1$ to $25.2 \pm 4.9$ for $\mathrm{H} 460(\mathrm{P}=0.014)$, resulting in an enhancement ratio (ER) of 1.23 and 1.49 respectively (Figure $9.1 \mathrm{~B}$ and Tables S9.1 and S9.2). In addition, the effect of TH302 was also assessed in the rhabdomyosarcoma model in combination with 4 and $12 \mathrm{~Gy}$ of radiotherapy leading to an ER of 1.28 and 1.59, respectively (Figure S9.3A and S9.3B and Table S9.1). TH-302 has a radio-sensitizing effect in both tumor models and all radiotherapy doses. Moreover, the effect of the combination therapy TH-302 and 12 Gy radiotherapy in the rhabdomyosarcoma model was even synergistic. 
A

Rhabdomyosarcoma

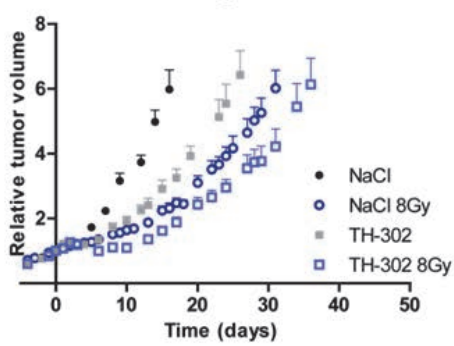

B

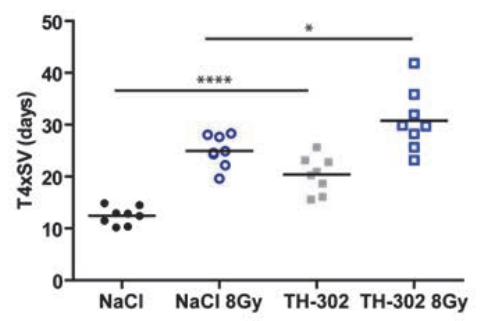

C
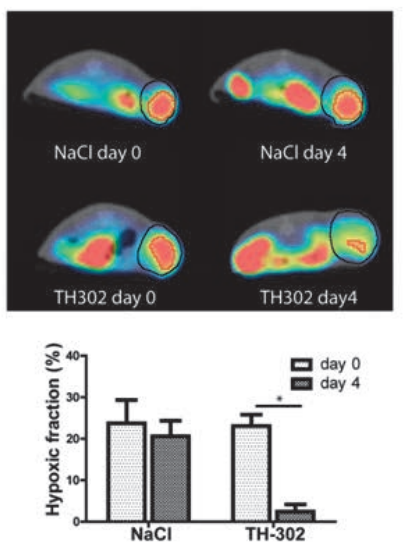

H460
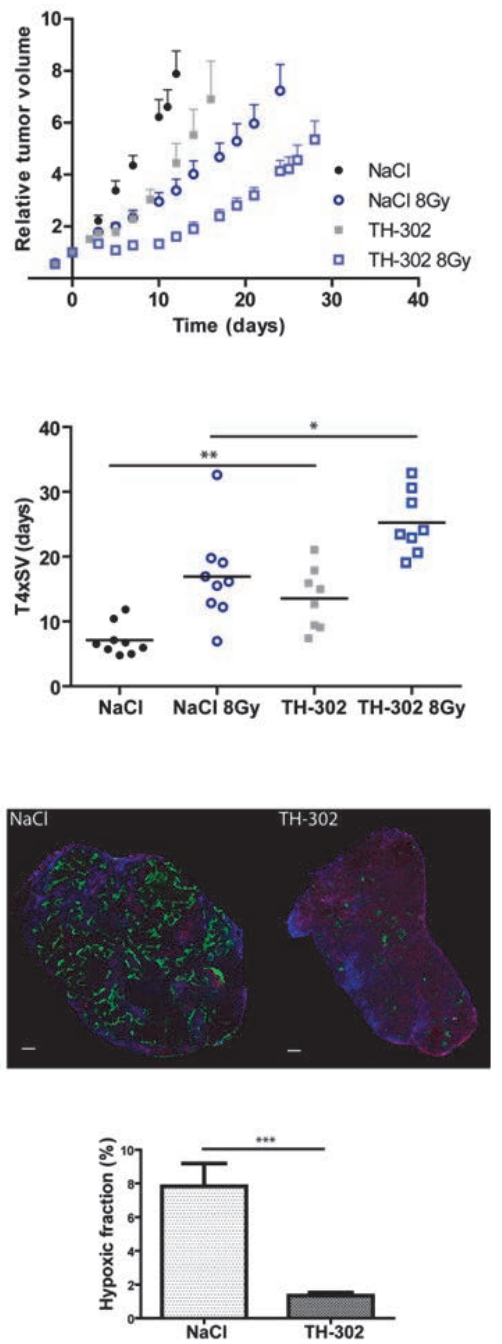

Figure 9.1: The combination of TH-302 and radiotherapy (8 Gy) reduces tumor growth (RT; 8Gy) in a rhabdomyosarcoma $(n=8)$ and $\mathrm{H} 460(n \geq 8)$ tumor model.A) growth curves; B) time to reach 4 times start volume (T4xSV). Animals were treated with either control ( $\mathrm{NaCl}$ ) or $\mathrm{TH}-302$ administered to the rhabdomyosarcoma model for 4 consecutive days with a dose of $25 \mathrm{mg} / \mathrm{kg}$ and for the $\mathrm{H} 460$ model for 5 consecutive days using a dose of 50 $\mathrm{mg} / \mathrm{kg}$. Radiotherapy was applied on either the third or fourth day of TH-302 treatment.The HF of the control animals and the animals treated with $\mathrm{TH}-302$ was analyzed in C) for rhabdomyosarcoma $(n=6)$ both before and immediately after treatment using $\left[{ }^{18} \mathrm{~F}\right] \mathrm{HX} 4$ hypoxia PET imaging (top, representative $\left[{ }^{18} \mathrm{~F}\right] \mathrm{HX} 4$ PET images with the delineation of the total tumor volume in black and the HF in red. bottom: quantification of HF per group) and in D) for $\mathrm{H460}(n=6)$ immediately after treatment using pimonidazole IHC staining [top: representative stainings, with hypoxia (pimonidazole) in green, perfusion (Hoechst) in blue and vessels (CD31) in red. The white scale bar indicates $500 \mu \mathrm{M}$. bottom: quantification]. Data, mean \pm SEM. ${ }^{*} P<0.05,{ }^{*} P<0.005,{ }^{* * *} P<0.001,{ }^{* * * *} P<0.0001$ 
A
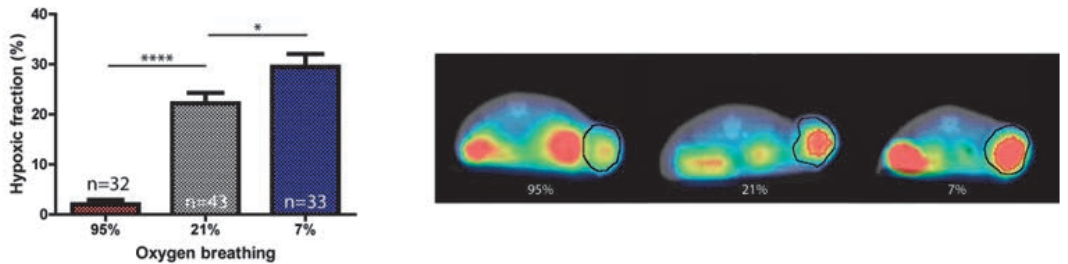

B

Rhabdomyosarcoma

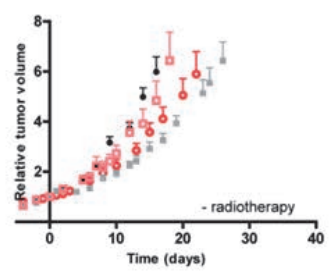

- $\mathrm{NaCl}$

- $\mathrm{NaCl} 95 \%$

- TH-302

D TH-30295\%

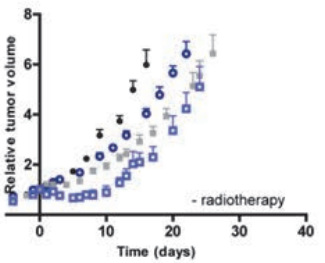

- $\mathrm{NaCl}$

- $\mathrm{NaCl} 7 \%$

- TH-302

- TH-3027\%
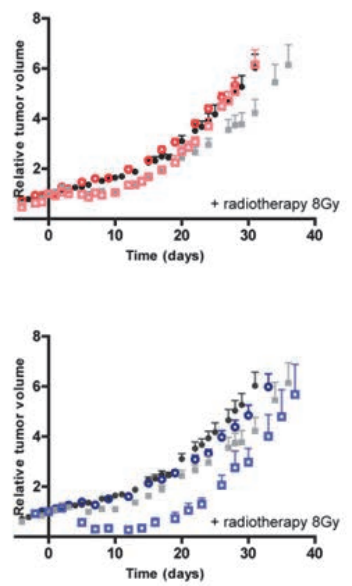

C

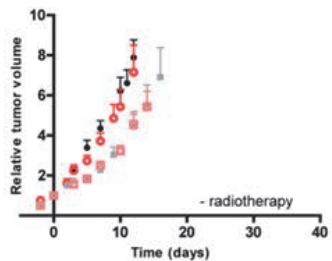

H460
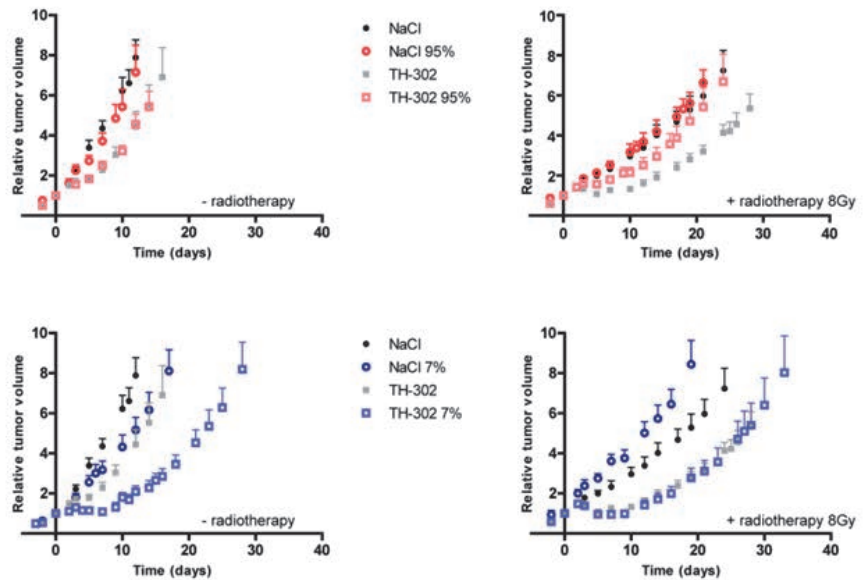

Figure 9.2: The effect of oxygen modification on the combination treatment of $\mathrm{TH}-302$ and radiotherapy. A) exposing animals to 95\% oxygen (nicotinamide $500 \mathrm{mg} / \mathrm{kg}$ i.p. / carbogen breathing 95\% oxygen, 5\% $\mathrm{CO}_{2}$ ), or $7 \%$ oxygen (residual $\mathrm{N}_{2}$ ) decreases or increases the hypoxic fraction respectively before the start of treatment compared to $21 \%$ oxygen breathing (ambient air). Representative $\left[{ }^{18} \mathrm{~F}\right] \mathrm{HX} 4 \mathrm{PET}$ images with the delineation of the tumor in black and the HF in red. ${ }^{*} P<0.05$, **** ${ }^{*}<0.0001$. Exposing the B) rhabdomyosarcoma $(n \geq 7)$ or $\left.C\right) H 460$ $(n \geq 8)$ model to modifying oxygen concentrations for the duration of the treatment for 4 and 2.5 hours per day, respectively. Data represent mean \pm SEM. 


\section{Hypoxic fraction}

The effect of TH-302 on the HF in the rhabdomyosarcoma model was assessed using [18F]HX4 hypoxia PET imaging and revealed a significant decrease from a baseline of $23.1 \% \pm 6.7$ to $2.5 \% \pm 4.2$ after $\mathrm{TH}-302$ treatment $(\mathrm{P}<0.001)$. $\mathrm{NaCl}$ treatment, as expected, did not affect the HF (Figure 9.1C). In the H460 model the HF was assessed immediately after the treatment using histological controls injected with pimonidazole. The HF in subjects treated with $\mathrm{TH}-302$ significantly decreased compared to the control animals ( $\mathrm{NaCl}: 7.8 \% \pm 3.0 ; \mathrm{TH}-302: 1.3 \% \pm 0.5$; Figure 9.1D).

\section{Effect of oxygen modification on the efficacy of TH-302 and radiotherapy}

To investigate if a causal relationship exists between TH-302 efficacy and tumor oxygenation, the amount of oxygen present in the tumor was modified on the days of $\mathrm{TH}-302$ treatment by $7 \%$ or $95 \%$ oxygen breathing to increase or decrease the HF, respectively. Oxygen modification was performed 1 day in advance for the rhabdomyosarcoma model in order to assess the effect of this modification on the baseline HF using [18F]HX4-PET imaging. Ambient air breathing animals had a HF of $22.2 \pm 13.8 \%$. A significant reduction in the $\mathrm{HF}$ to $2.1 \pm 4.7 \%$ was seen after $95 \%$ oxygen breathing $(P<0.001)$, whereas $7 \%$ oxygen breathing significantly increased the HF to $29.5 \pm 14.7 \%(P=0.029$; Figure 9.2A). Exposing rhabdomyosarcoma-bearing rats to increasing oxygen conditions abolished the effect of TH-302 and reduced the T4XSV from $20.4 \pm 3.5$ to $15.3 \pm 2.5$ days $(P=0.007$, Figure 9.2B, Figure S9.3C and Table S9.1), whereas control animals had an increased T4xSV. Upon combination with radiotherapy, the T4xSV of TH-302 treated tumors decreased from 30.8 \pm 5.9 ( $\mathrm{TH}-302+$ radiotherapy) to $25.7 \pm 2.9$ days ( $\mathrm{TH}-302+$ radiotherapy $+95 \% \mathrm{O} 2)$. This is comparable with the T4xSV of $23.2 \pm 1.7$ days for animals treated with $\mathrm{NaCl}+$ radiotherapy under $95 \% \mathrm{O} 2$ conditions resulting in an ER of 1.11 (Table S9.1). Exposing animals to $7 \%$ oxygen breathing resulted in a T4xSV of $22.6 \pm 4.2$ days for TH-302 which is significantly delayed compared to the animals treated with $\mathrm{NaCl}(\mathrm{T} 4 \times \mathrm{SV}: 16.1 \pm 1.9, \mathrm{P}=0.001)$ although $7 \%$ oxygen treatment itself also had an effect on tumor growth in control animals. In the combination therapy of 7\% oxygen breathing with radiotherapy, animals treated with $\mathrm{TH}$ 302 had a further reduction in the T4XSV to $35.4 \pm 6.1$ days with an ER of 1.29 compared to the animals treated with $\mathrm{NaCl}$ (Figure S9.3C and Table S9.1).

Oxygen modification treatments were also applied to the $\mathrm{H} 460$ model where reducing the $\mathrm{HF}$ resulted in a decreased T4xSV from $25.2 \pm 4.9$ (TH-302 + radiotherapy) to $20.2 \pm 7.0$ (TH-302 + radiotherapy $+95 \%$ O2) for the combination treatment. This decrease was not significant, however. The ER of TH-302 under high oxygen concentration remained stable at 1.50 versus 1.49 at $21 \% \mathrm{O} 2$ breathing. The tumor growth rate itself was unaffected by 95\% oxygen breathing (Figure 9.2C, Figure S9.3D and Table S9.2). Increasing the HF significantly enlarged the therapeutic potential of TH-302 compared to normal breathing animals $(P=0.011)$, resulting in a T4xSV of $22.7 \pm 7.9$ (T4xSV TH-302 21\% 02: $13.6 \pm 4.8$ ). While $7 \%$ oxygen breathing reduced the tumor growth slightly, radiotherapy increased the tumor growth of control animals under this condition. 
174 Chapter 9

A

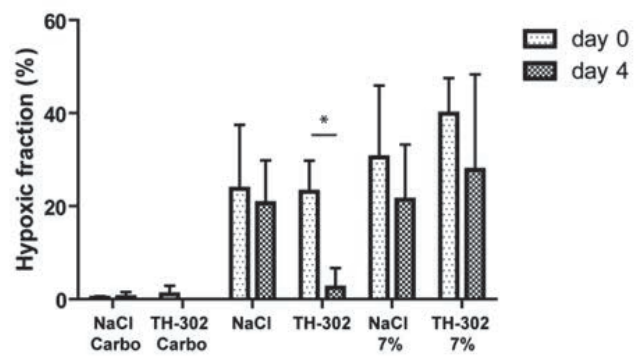

B
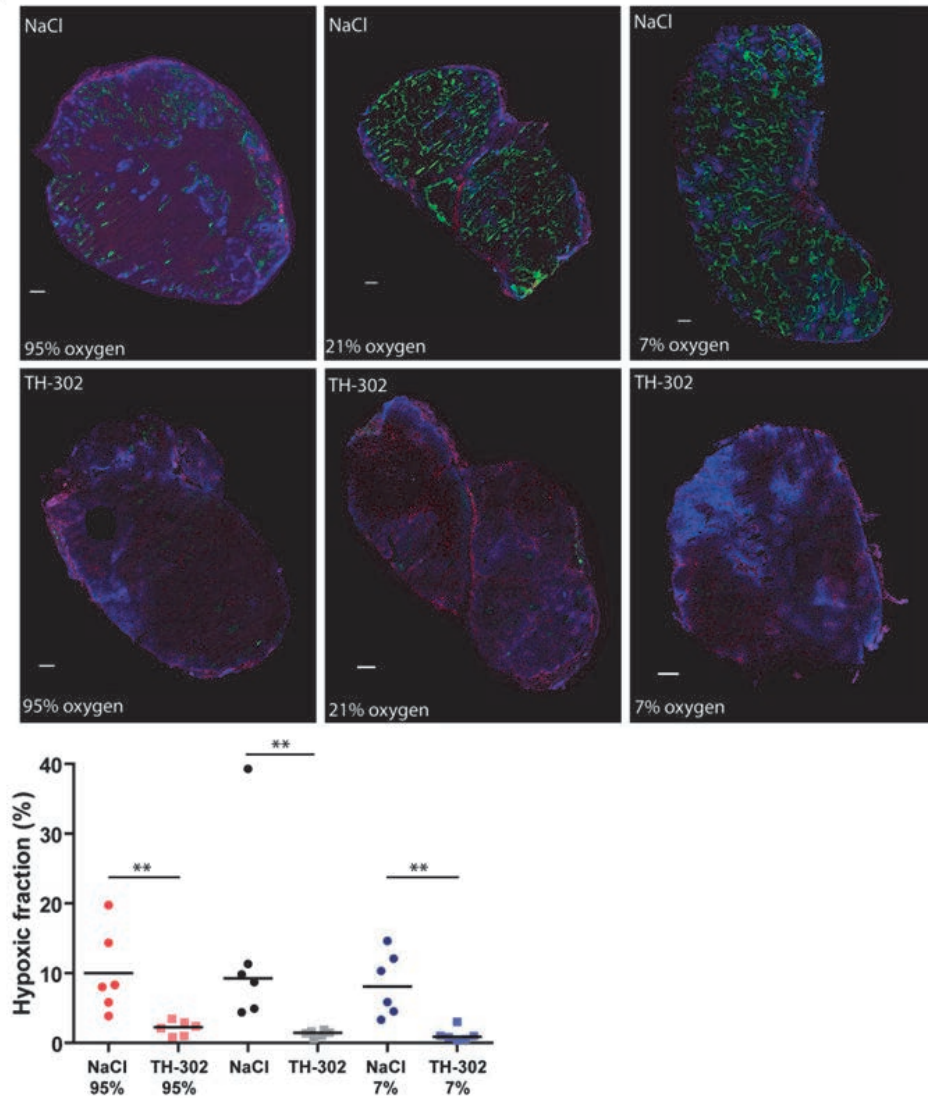

Figure 3: The effect of $\mathrm{TH}-302$ treatment and oxygen modification on the HF. A) HF was measured in the rhabdomyosarcoma model $(n=6)$ using $\left[{ }^{18} \mathrm{~F}\right] \mathrm{HX} 4$ hypoxia PET imaging the day before treatment and the last day of treatment with either control $(\mathrm{NaCl})$ or $\mathrm{TH}-302$ in combination with 95\% oxygen (nicotinamide and $95 \% \mathrm{O}_{2}$ breathing), ambient air or $7 \%$ oxygen. ND = not detected. B) pimonidazole staining was used to determine the HF after treatment in the $\mathrm{H} 460$ model. Top: a representative image is depicted per group. Bottom: quantification per group $(n=6)$. ${ }^{* *} P<0.01, * * * P<0.001$ 
The effect of the TH-302 and radiotherapy combination increased to an ER of 2.45 for TH$302+$ radiotherapy under low oxygen concentrations versus 1.49 for $\mathrm{TH}-302$ + radiotherapy under $21 \% \mathrm{O} 2$ concentrations (Figure S9.3D and Table S9.2).

No toxic effects were observed for the different treatments in any of the groups as monitored by following changes in body weight (Figure S9.4A and S9.4B).

\section{Oxygen modification and hypoxic fraction}

To assess the effect of $\mathrm{TH}-302$ treatment in combination with oxygen modification on the $\mathrm{HF}$, a [18F]HX4 scan was acquired before and after treatment on the rhabdomyosarcoma histological control animals. The HF of ambient air breathing animals decreased from $23 \%$ $\pm 6.7 \%$ at baseline to $2.5 \% \pm 4.2 \%$ after $\mathrm{TH}-302$ treatment. For $95 \%$ oxygen breathing animals the HF was low before the start of the treatment, and this remained unchanged after either $\mathrm{NaCl}$ or $\mathrm{TH}-302$ administration (Figure 9.3A). The spread in $\mathrm{HF}$ of $7 \%$ oxygen breathing animals was very large. On average the HF after treatment was lower than before treatment independent of $\mathrm{NaCl}$ or $\mathrm{TH}-302$ treatment although this was not significant.

The HF in the $\mathrm{H} 460$ model was determined after the last $\mathrm{TH}-302$ injection using pimonidazole staining. TH-302 treatment significantly reduced the HF compared to the control animals (Figure 9.1D and Figure 9.3B). The different oxygen breathing conditions revealed a similar pattern; in combination with $95 \%$ oxygen breathing control animals had a $\mathrm{HF}$ of $10.0 \% \pm 5 \% .9$ while animals treated with $\mathrm{TH}-302$ had a HF of $2.1 \% \pm 1.0 \%$. Animals exposed to low oxygen concentrations in combination with $\mathrm{NaCl}$ had a $\mathrm{HF}$ of $8.4 \% \pm 4.5 \%$ which was lower in the animals treated with TH-302 (1.1\% $\pm 1.0 \%)$. Furthermore, TH-302treated tumors had a decreased necrotic fraction, although this was only significant when animals were exposed to $21 \%$ oxygen. No differences were observed in the relative vessel area or perfusion (Figure S9.5).

Furthermore, we investigated whether the HF at the start of the therapy was associated with the treatment outcome expressed as T4xSV. The T4xSV for TH-302 treated tumors increased with increasing HF at onset meaning that 95\% oxygen breathing animals reached their endpoint first, followed by ambient air and then $7 \%$ oxygen breathing animals (Figure 9.4). The control animals, with or without radiotherapy, did not show this association. 

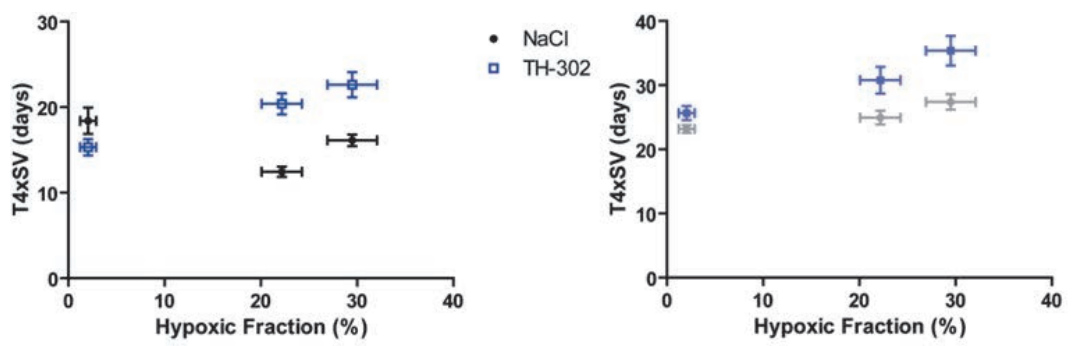

Figure 9.4: The association between the pretreatment $\left[{ }^{18} \mathrm{~F}\right] \mathrm{HX} 4$-based HF and the treatment outcome in rhabdomyosarcoma tumors. HF (\%) as measured before the start of the treatment plotted to the time to reach 4 times the start volume (T4xSV) for the control group ( $\mathrm{NaCl}$ ) and animals treated with $\mathrm{TH}-302$ either with or without radiotherapy. Hypoxic fraction is determined using $\left[{ }^{18} \mathrm{~F}\right] \mathrm{HX} 4$ hypoxia PET imaging after 95\% oxygen breathing ( $n=$ $32)$, ambient air breathing $(n=43)$ and $7 \%$ oxygen breathing $(n=33)$. The T4xSV is calculated for $\mathrm{NaCl}$ for all oxygen concentrations using 8 animals, TH-302 95\% oxygen $(n=7)$, ambient air and $7 \%$ oxygen $(n=8)$. Data represent mean \pm SEM.

\section{DISCUSSION}

This study demonstrates the combination efficacy of the HAP TH-302 with radiotherapy in two preclinical tumor models, which was causally related to the tumor oxygen status. Additionally, the $\left[{ }^{18} \mathrm{~F}\right] \mathrm{HX} 4$ determined HF was associated with the treatment outcome.

Pharmacokinetic studies in nontumor-bearing rats showed no adverse effects when the animals were treated with $\mathrm{TH}-302$ (17). Although rhabdomyosarcoma-bearing rats showed no adverse effect after a $25 \mathrm{mg} / \mathrm{kg}$ TH-302 dose, dose-dependent adverse effects, such as a significant drop in body weight, diarrhea and general malaise, were observed after higher dosing. Initial clinical studies also reported some adverse effects with skin and mucosal toxicities being dose limiting, while common adverse events were nausea, skin rash, fatigue and vomiting $(5,6)$.

In this study two different methods were used to assess the HF: noninvasive, clinically used $\left[{ }^{18} \mathrm{~F}\right] \mathrm{HX} 4$ PET imaging $(11,13)$, and IHC of pimonidazole adducts (9). While $\left[{ }^{18} \mathrm{~F}\right] \mathrm{HX} 4$ hypoxia PET imaging represents the whole tumor in a noninvasive, reproducible, threedimensional manner, IHC stainings can, in addition to hypoxia, extract more tumor microenvironmental information from the same tumor section. In both techniques, $\left[{ }^{18} \mathrm{~F}\right] \mathrm{HX} 4$ and pimonidazole, are reduced under low oxygen concentrations (18) and a significant colocalization relationship was demonstrated at the tumor sub-regional level (9). Although monitored by different techniques, these data indicate that $\mathrm{TH}-302$ has the same reducing effect on the HF in both tumor models. Furthermore, based on these data, $\left[{ }^{18} \mathrm{~F}\right] \mathrm{HX} 4$ imaging could be used as biomarker of response in a window-of-opportunity clinical trial. By performing a pretreatment scan, the initial tumor HF can be determined, followed by a single injection of $\mathrm{TH}-302$. A posttreatment $\left[{ }^{18} \mathrm{~F}\right] \mathrm{HX} 4$ hypoxia scan can assess whether there is a response in HF to this HAP, without interference of other anticancer therapies. This new approach is designed to test new molecular entities in a clinical trial while being cost and patient efficient $(19,20)$. 
A correlation has previously been reported between the HF and the tumor growth inhibition in xenograft models (3). This endorses our findings of a pronounced effect of $\mathrm{TH}-302$ since the preclinical tumor models in this study were observed to have respectively somewhat higher and lower HF than the reported HF of $18.6 \%$ for rhabdomyosarcoma (15) and $16.3 \%$ for H460 (3). Although the HF in tumors was significantly decreased after TH-302 treatment, not all hypoxic cells were eliminated, as observed in both tumor models. The remaining hypoxic cells might be resistant, unreachable by $\mathrm{TH}-302$, or caused by cycling hypoxia. In addition to the decrease in the HF, this study shows a decrease in the necrotic fraction of the TH-302 treated tumors. This indicates that the dead cells are resorbed, which is supported by the stagnation in tumor growth after 3 days of TH-302 treatment. Other microenvironmental characteristics like relative vessel area and perfusion were not affected by $\mathrm{TH}-302$ treatment, suggesting that the tumor maintains its vasculature. This is in agreement with previously published results on solid tumors (3).

Although TH-302 exhibits antitumor effects as a monotherapy, it has been shown that its therapeutic efficacy increases when combined with conventional anticancer therapies mainly targeting the nonhypoxic cells. On the basis of favorable outcomes of two clinical phase II trials $(7,8)$, phase III trials in metastatic or locally advanced unresectable pancreatic adenocarcinoma (NCT01746979) and advanced soft-tissue sarcoma (NCT01440088) are currently ongoing. However, to our knowledge, no study has investigated the combination treatment of $\mathrm{TH}-302$ with radiotherapy and $\left[{ }^{18} \mathrm{~F}\right] \mathrm{HX} 4$ hypoxia imaging. This combination is thought to be effective especially since radiotherapy can be locally applied, specifically targeting the tumor while preserving normal tissue. In this study we show that this combination is effective and causes a delayed tumor growth and increased T4xSV for both investigated tumor models, confirming the hypothesis that the combination therapy of TH-302 and radiotherapy will lead to an enhanced therapeutic effect. In the rhabdomyosarcoma tumor model, TH-302 treatment was combined with a single dose of 4, 8 and 12 Gy of radiotherapy resulting in a dose dependent effect. In subsequent studies the single radiotherapy dose of 8 Gy was used, reasoning that the regrowth of the tumor solely depends on hypoxic cells (21), providing a basis for TH-302 efficacy. This approach is different from clinical practice where fractionated radiotherapy schedules are used. By applying $2 \mathrm{~Gy}$ fractions to the tumor, reoxygenation occurs and the HF gradually decreases $(22,23)$. We speculate that the combination of TH-302 with fractionated radiotherapy would also increase the therapeutic effect of the radiotherapy since the HF is reduced by the pretreatment of $\mathrm{TH}-302$, increasing the potential of radiotherapy.

In this study we further wanted to elucidate if TH-302 efficacy is dependent on the tumor oxygen status. Exposing animals to either nicotinamide and carbogen or $7 \%$ oxygen breathing has been demonstrated to be effective in modulating the HF in tumors $(9,24$, 25). Altering oxygen breathing before the TH-302 treatment did modify the tumor HF in rhabdomyosarcoma animals as measured by $\left[{ }^{18} \mathrm{~F}\right] \mathrm{HX} 4$. However, in the $\mathrm{H} 460$ model, the HF was only determined after TH-302 treatment and at this point no differences were observed in control animals of the various oxygen modifications. A possible explanation would be that the mice adapted to the oxygen breathing schedule, preventing the tumor HF from changing, which has been observed for mice exposed to long-term carbogen breathing $(26,27)$. In the rhabdomyosarcoma model, the tumor growth of control animals 
was significantly reduced upon oxygen modification as well as in the mice exposed to $7 \%$ oxygen breathing. No effect on tumor growth was observed after oxygen modification in another study using $\mathrm{H} 460$ tumors exposed to $95 \%$ or $10 \%$ oxygen breathing (3). This unexpected finding could possibly be explained by the stress induced by the exposure to the oxygen modifications, although $\mathrm{TH}-302$ or radiotherapy treatment groups did not seem to be influenced by this. However, by calculating the enhancement ratio these oxygen modification effects are taken into account. Despite these effects on growth delay a positive effect of the therapy is observed. Radiotherapy was applied 1 hour after carbogen breathing without any beneficial effect. This can be explained by the fact that tumor oxygen concentrations return to pre-carbogen levels within 1 minute after stopping carbogen treatment as detected by Eppendorf electrode measurements (28). Furthermore, clinical studies have shown that the presence of hypoxia and the pretreatment selection of patients with hypoxic tumors is essential for the combination of nicotinamide administration and carbogen breathing to be effective $(29,30)$. Breathing low oxygen concentrations reduced the effect of radiotherapy in the $\mathrm{H} 460$ model, indicating that, although not detected on pimonidazole immunohistochemical staining, low oxygen concentrations counteracted the irradiation. The effect of TH-302 is abolished by carbogen breathing in the rhabdomyosarcoma model independent of radiotherapy. This can be explained by the reduced HF leaving almost no cells present to convert TH-302 into its cytotoxic metabolite. For the $\mathrm{H} 460$ model the HF did not decrease upon carbogen breathing what reflects in the unchanged tumor growth compared to control tumors. Upon radiotherapy however, there is a trend towards a faster tumor growth that also indicating abolishment of the TH-302 efficacy. In tumors with an enlarged HF, TH-302 caused a slight, non-significant, delay in tumor growth compared to TH-302 under normal air conditions. Moreover, TH-302 decreased the HF to almost zero under ambient air conditions, while with $7 \%$ oxygen breathing the $\mathrm{HF}$ is still $28 \%$. Although this result could be caused by the counteracting effects of $\mathrm{TH}-302$ reducing the $\mathrm{HF}$ and the $7 \%$ oxygen breathing increasing the HF, we speculate that it is caused by a limited availability of $\mathrm{TH}-302$ to target all hypoxic cells. In $\mathrm{H} 460$ tumors, 7\% oxygen breathing resulted in an increased therapeutic effect with an enhancement ratio of 2.2 for $\mathrm{TH}-302$ alone and 2.5 for the combination therapy of TH-302 and radiotherapy. This result demonstrates that when sufficient TH-302 is present, more $\mathrm{TH}-302$ is reduced upon low oxygen concentrations, causing an increased cytotoxicity.

A causal relation between the pretreatment $\mathrm{HF}$ measured by $\left[{ }^{18} \mathrm{~F}\right] \mathrm{HX} 4$ and the $\mathrm{TH}-302$ treatment outcome was observed. These results indicate that pretreatment evaluation of hypoxia could be a useful tool in selecting tumors that benefit from the additional hypoxia targeting treatment. This hypoxia-based patient selection could also be used in other therapy strategies for instance to target hypoxic subvolumes by escalate radiation dose (31). Furthermore this information could be implemented in decision-support systems to predict tumor response and optimize patient therapy (32). These applications demonstrate the importance of gaining pretreatment information by hypoxia imaging. 


\section{CONCLUSION}

This study demonstrates that TH-302 treatment together with conventional radiotherapy is a promising combination with an increased therapeutic potential, and warrants further testing. Furthermore, detecting the tumor hypoxic fraction by $\left[{ }^{18} \mathrm{~F}\right] \mathrm{HX} 4 \mathrm{PET}$ imaging may allow the ability to predict which patients will benefit most from the hypoxia targeted $\mathrm{TH}-$ 302 treatment and gives the possibility to noninvasively monitor $\mathrm{TH}-302$ efficacy in the context of window-of-opportunity trials. Based on this preclinical study we suggest a clinical trial for treating patients with the combination of $\mathrm{TH}-302$ and radiotherapy while monitoring the hypoxic fraction before and during the treatment.

\section{ACKNOWLEDGEMENTS}

Authors acknowledge financial support from the QulC-ConCePT project, which is partly funded by EFPI A companies and the Innovative Medicine Initiative Joint Undertaking (IMI JU) under Grant Agreement No. 115151. Authors also acknowledge financial support from the EU 6th and 7th framework program (METOXIA, EURECA, ARTFORCE), Kankeronderzoekfonds Limburg from the Health Foundation Limburg and the Dutch Cancer Society (KWF UM 2011-5020, KWF MAC 2013-6425, KWF MAC 2013-6089). The micrographs in this paper were taken with a confocal spinning disk microscope financed by The Netherlands Organization for Scientific Research (NWO), grant number 911-06-003. 


\section{SUPPLEMENTARY DATA}

Rhabdomyosarcoma $\quad 0 \quad 3 \quad 3 \quad 3 \quad$ Time (days)

\begin{tabular}{|l|c|c|c|}
\hline Treatment group & HX4 & $\mathrm{NaCl} / \mathrm{TH}-302$ & Follow up tumor growth \\
\hline Histological controls & $\mathrm{H} \times 4$ & $\mathrm{NaCl} / \mathrm{TH}-302$ & \\
\hline \multirow{3}{*}{ Oxygen modification } & Nicotinamide / Carbogen (95\% oxygen) \\
\hline
\end{tabular}

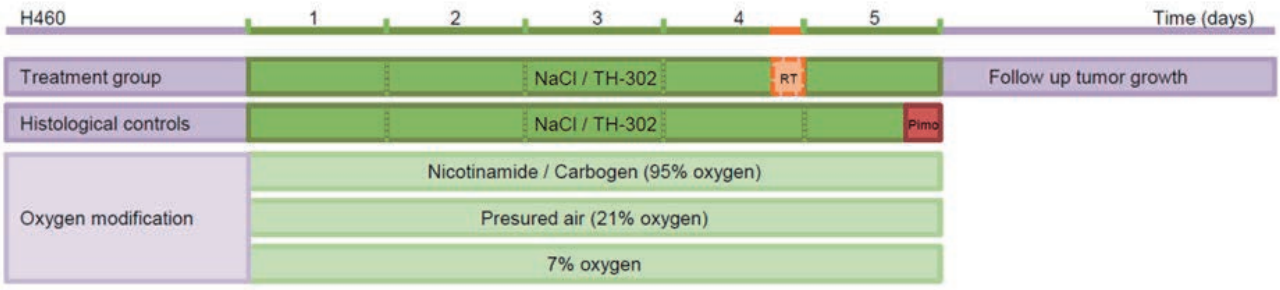

Figure 59.1: Treatment schedule for the rhabdomyosarcoma and $\mathrm{H} 460$ models. All animals were randomized over either the treatment or the control group. These groups were both treated with either NaCl or TH-302 (rhabdomysarcoma $25 \mathrm{mg} / \mathrm{kg}, \mathrm{H} 46050 \mathrm{mg} / \mathrm{kg}$ ). Rhabdomyosarcoma bearing animals started with oxygen modification at day 0 and a [18F]HX4 hypoxia PET scan. For histological controls only, this scan was repeated on day 4. Animals from the treatment group were exposed to radiotherapy (RT) at the end of treatment day 3 and their tumor volume was monitored until 4 times start volume was reached. $\mathrm{H} 460$ tumor bearing animals from the histological control group were injected with pimonidazole and Hoechst on the last treatment day and sacrificed. Animals from the treatment group were exposed to radiotherapy at day 4 and monitored until a tumor volume of 4 times start treatment volume was reached.
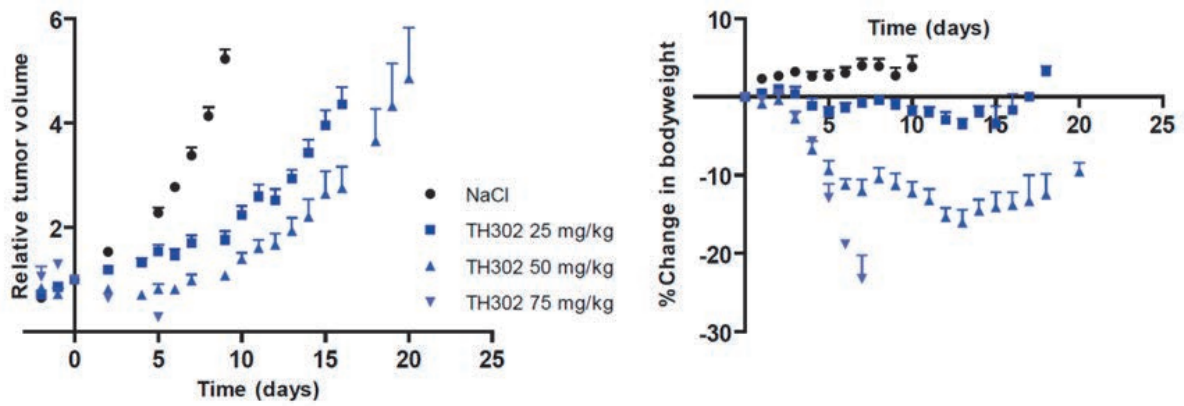

Figure 59.2: Optimal TH-302 dose assessment for rhabdomyosarcoma bearing rats. Animals were treated on 4 consecutive days with either the control ( $\mathrm{NaCl}), 25,50$ or $75 \mathrm{mg} / \mathrm{kg} \mathrm{TH-302.} \mathrm{Tumor} \mathrm{volume} \mathrm{A)} \mathrm{and} \mathrm{body} \mathrm{weight} \mathrm{B)}$ was monitored over time. Data represent mean \{plus minus\} SEM, for all groups $n=9$. 
A

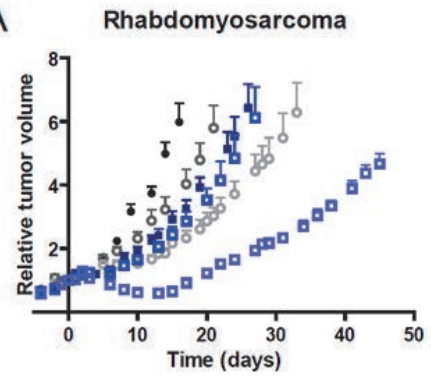

C

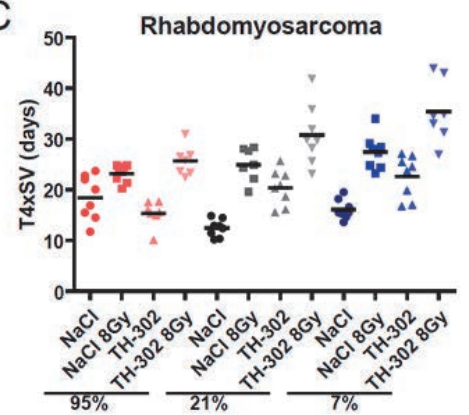

B

- $\mathrm{NaCl}$

- $\mathrm{NaCl} 4 \mathrm{~Gy}$

- $\mathrm{NaCl} 12 \mathrm{~Gy}$

- $\mathrm{TH}-302$

- $\mathrm{TH}-3024 \mathrm{G}$.

- $\mathrm{TH}-30212 \mathrm{C}$

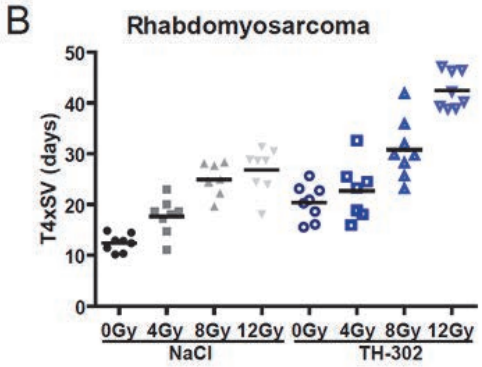

D

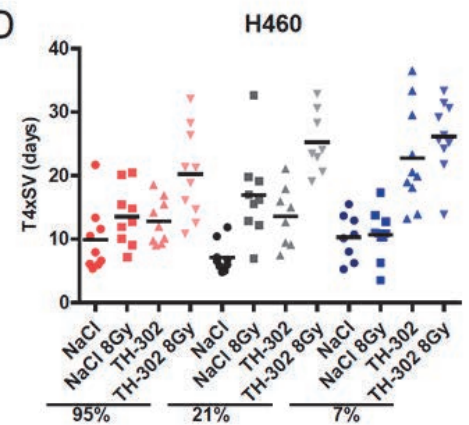

Figure 59.3: A) Tumor growth of rhabdomyosarcoma bearing animals $(n=8)$ treated with TH-302 $(25 \mathrm{mg} / \mathrm{kg})$ in combination with 4, 8 or 12Gy of RT. Data represent mean \{plus minus\} SEM. B) T4xSV for rhabdomyosarcoma bearing animals treated with TH-302 in combination with RT 0, 4, 8 or 12Gy. C) T4xSV for rhabdomyosarcoma bearing animals exposed to different oxygen concentrations in combination with TH-302 and RT. D) T4xSV for H460 bearing animals exposed to different oxygen concentrations in combination with TH-302 and RT. 
182 Chapter 9

A

Rhabdomyosarcoma
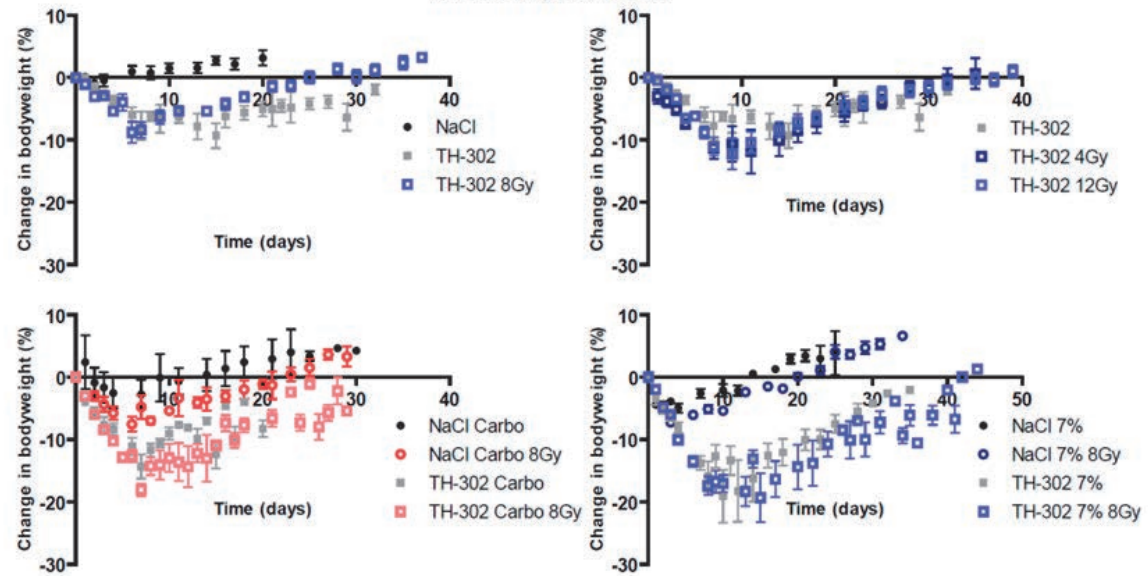

B

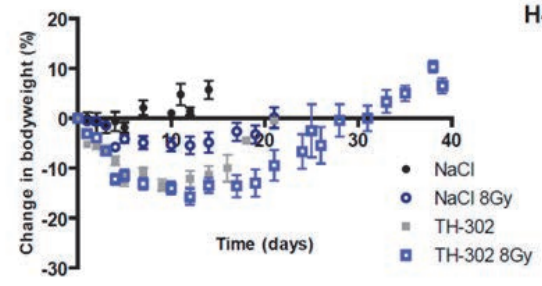

H460
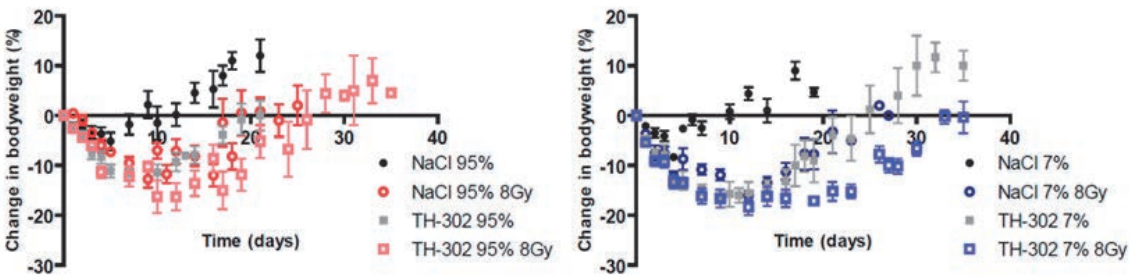

Figure S9.4: Body weight was monitored during and after treatment for both rhabdomyosarcoma A) and $\mathrm{H} 460 \mathrm{~B}$ ) tumor-bearing animals. The change in body weight is represented in percentage. Data represent mean \pm SEM. 
A

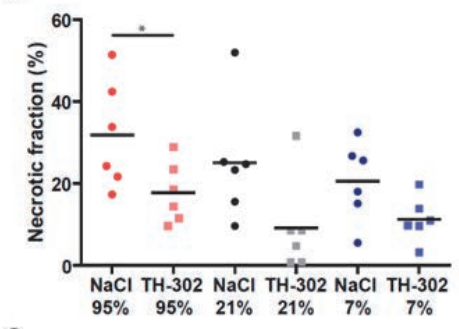

C

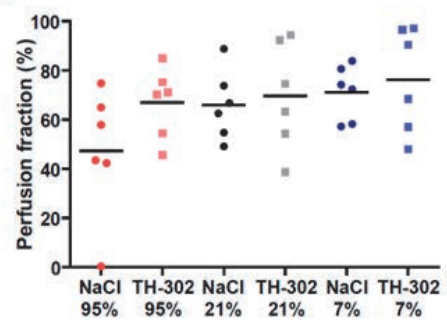

B

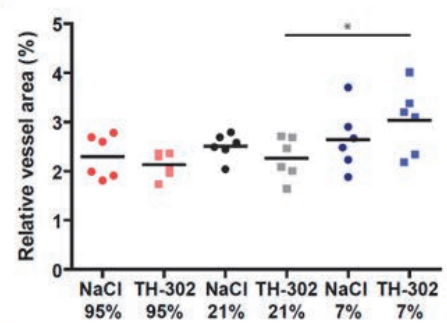

D

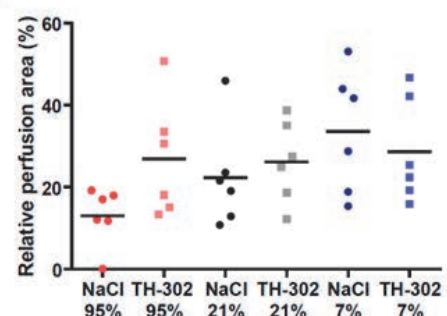

Figure S9.5: Quantification of $\mathrm{H} 460$ histological staining for A) necrotic fraction (NF), B) relative vessel area (RVA), C) perfusion fraction (PF) and D) relative perfusion area (RPA). Data represent mean \pm SEM.

Table S9.1: Time to reach 4 times start volume per treatment group for rhabdomyosarcoma bearing animals. Data represent mean $\pm S D, p$-value and sensitization enhancement ratio.

\begin{tabular}{lllll}
\hline Rhabdomyosarcoma & $\begin{array}{l}\text { T4xSV } \\
\text { NaCl }\end{array}$ & $\begin{array}{l}\text { T4xSV } \\
\text { TH-302 }\end{array}$ & P-value & ER \\
\hline $21 \%$ & $12.4 \pm 1.7$ & $20.4 \pm 3.5$ & $<0.0001$ & 1.64 \\
$21 \%$ RT 4Gy & $17.7 \pm 3.6$ & $22.7 \pm 5.6$ & 0.0583 & 1.28 \\
$21 \%$ RT 8Gy & $24.9 \pm 3.0$ & $30.8 \pm 5.9$ & 0.0261 & 1.23 \\
$21 \%$ RT 12Gy & $26.8 \pm 4.4$ & $42.5 \pm 3.6$ & $<0.0001$ & 1.58 \\
$95 \%$ & $18.4 \pm 4.4$ & $15.3 \pm 2.5$ & 0.1234 & 0.83 \\
$95 \%$ RT & $23.2 \pm 1.7$ & $25.7 \pm 2.9$ & 0.0601 & 1.11 \\
$7 \%$ & $16.1 \pm 1.9$ & $22.6 \pm 4.2$ & 0.0013 & 1.40 \\
$7 \%$ RT & $27.4 \pm 3.4$ & $35.4 \pm 6.1$ & 0.0072 & 1.29 \\
\hline
\end{tabular}

Table 59.2: Time to reach 4 times start volume per treatment group for H460 bearing animals. Data represent mean $\pm S D, p$-value and sensitization enhancement ratio.

\begin{tabular}{lllll}
\hline H460 & T4xSV & T4xSV & P-value & ER \\
& NaCl & TH-302 & & \\
\hline $21 \%$ & $7.1 \pm 2.4$ & $13.6 \pm 4.8$ & 0.0027 & 1.90 \\
$21 \%$ RT & $16.9 \pm 7.1$ & $25.2 \pm 4.9$ & 0.0136 & 1.49 \\
$95 \%$ & $9.9 \pm 5.2$ & $12.8 \pm 3.6$ & 0.1917 & 1.29 \\
$95 \%$ RT & $13.5 \pm 4.6$ & $20.2 \pm 7.0$ & 0.0260 & 1.50 \\
$7 \%$ & $10.3 \pm 3.6$ & $22.7 \pm 7.9$ & 0.0009 & 2.21 \\
$7 \%$ RT & $10.7 \pm 3.8$ & $26.2 \pm 5.6$ & $<0.0001$ & 2.45 \\
\hline
\end{tabular}




\section{REFERENCES}

1. Horsman MR, Mortensen LS, Petersen JB, Busk M, Overgaard J. Imaging hypoxia to improve radiotherapy outcome. Nature reviews Clinical oncology. 2012;9:674-87.

2. Vaupel P, Mayer A. Hypoxia in cancer: significance and impact on clinical outcome. Cancer Metastasis Rev. 2007;26:225-39.

3. Sun JD, Liu Q, Wang J, Ahluwalia D, Ferraro D, Wang Y, et al. Selective tumor hypoxia targeting by hypoxia-activated prodrug $\mathrm{TH}-302$ inhibits tumor growth in preclinical models of cancer. Clin Cancer Res. 2012;18:758-70.

4. Liu Q, Sun JD, Wang J, Ahluwalia D, Baker AF, Cranmer LD, et al. TH-302, a hypoxiaactivated prodrug with broad in vivo preclinical combination therapy efficacy: optimization of dosing regimens and schedules. Cancer Chemother Pharmacol. 2012;69:1487-98.

5. Weiss GJ, Infante JR, Chiorean EG, Borad MJ, Bendell JC, Molina JR, et al. Phase 1 study of the safety, tolerability, and pharmacokinetics of TH-302, a hypoxia-activated prodrug, in patients with advanced solid malignancies. Clin Cancer Res. 2011;17:2997-3004.

6. Ganjoo KN, Cranmer LD, Butrynski JE, Rushing D, Adkins D, Okuno SH, et al. A phase I study of the safety and pharmacokinetics of the hypoxia-activated prodrug $\mathrm{TH}-302$ in combination with doxorubicin in patients with advanced soft tissue sarcoma. Oncology. 2011;80:50-6.

7. Chawla SP, Cranmer LD, Van Tine BA, Reed DR, Okuno SH, Butrynski JE, et al. Phase II Study of the Safety and Antitumor Activity of the Hypoxia-Activated Prodrug TH-302 in Combination With Doxorubicin in Patients With Advanced Soft Tissue Sarcoma. J Clin Oncol. 2014.

8. Borad MJ, Reddy SG, Bahary N, Uronis HE, Sigal D, Cohn AL, et al. Randomized Phase II Trial of Gemcitabine Plus TH-302 Versus Gemcitabine in Patients With Advanced Pancreatic Cancer. J Clin Oncol. 2014.

9. Dubois LJ, Lieuwes NG, Janssen MH, Peeters WJ, Windhorst AD, Walsh JC, et al. Preclinical evaluation and validation of [18F]HX4, a promising hypoxia marker for PET imaging. Proc Natl Acad Sci U S A. 2011;108:14620-5.

10. Zegers $C M$, van Elmpt W, Wierts $R$, Reymen B, Sharifi H, Ollers MC, et al. Hypoxia imaging with $[(1)(8) F] H X 4$ PET in NSCLC patients: defining optimal imaging parameters. Radiother Oncol. 2013;109:58-64.

11. Zegers CM, van Elmpt W, Reymen B, Even AJ, Troost EG, Ollers MC, et al. In Vivo Quantification of Hypoxic and Metabolic Status of NSCLC Tumors Using [18F]HX4 and [18F]FDG-PET/CT Imaging. Clin Cancer Res. 2014.

12. Rischin D, Hicks RJ, Fisher R, Binns D, Corry J, Porceddu S, et al. Prognostic significance of [18F]-misonidazole positron emission tomography-detected tumor hypoxia in patients with advanced head and neck cancer randomly assigned to chemoradiation with or without tirapazamine: a substudy of Trans-Tasman Radiation Oncology Group Study 98.02. J Clin Oncol. 2006;24:2098-104.

13. Peeters SG, Zegers CM, Lieuwes NG, van Elmpt W, Eriksson J, van Dongen GA, et al. A Comparative Study of the Hypoxia PET Tracers [F]HX4, [F]FAZA, and [F]FMISO in a Preclinical Tumor Model. Int J Radiat Oncol Biol Phys. 2014. 
14. van Loon J, Janssen $M H$, Ollers $M$, Aerts HJ, Dubois L, Hochstenbag $M$, et al. PET imaging of hypoxia using [18F]HX4: a phase I trial. Eur J Nucl Med Mol Imaging. 2010;37:1663-8.

15. Dubois L, Landuyt $W$, Haustermans K, Dupont P, Bormans G, Vermaelen $P$, et al. Evaluation of hypoxia in an experimental rat tumour model by [(18)F]fluoromisonidazole PET and immunohistochemistry. Br J Cancer. 2004;91:1947-54.

16. Edelstein A, Amodaj N, Hoover K, Vale R, Stuurman N. Computer control of microscopes using microManager. Curr Protoc Mol Biol. 2010;Chapter 14:Unit14 20.

17. Jung D, Lin L, Jiao H, Cai X, Duan JX, Matteucci M. Pharmacokinetics of TH-302: a hypoxically activated prodrug of bromo-isophosphoramide mustard in mice, rats, dogs and monkeys. Cancer Chemother Pharmacol. 2012;69:643-54.

18. Wilson WR, Hay MP. Targeting hypoxia in cancer therapy. Nat Rev Cancer. 2011;11:393-410.

19. Fass L. Imaging and cancer: a review. Mol Oncol. 2008;2:115-52.

20. Orloff J, Douglas F, Pinheiro J, Levinson S, Branson M, Chaturvedi P, et al. The future of drug development: advancing clinical trial design. Nat Rev Drug Discov. 2009;8:949-57.

21. Hill RP, Bush RS, Yeung P. The effect of anaemia on the fraction of hypoxic cells in an experimental tumour. Br J Radiol. 1971;44:299-304.

22. Stanley JA, Shipley WU, Steel GG. Influence of tumour size on hypoxic fraction and therapeutic sensitivity of Lewis lung tumour. Br J Cancer. 1977;36:105-13.

23. Wouters BG, Brown JM. Cells at intermediate oxygen levels can be more important than the "hypoxic fraction" in determining tumor response to fractionated radiotherapy. Radiat Res. 1997;147:541-50.

24. Horsman MR, Overgaard J. Preclinical studies on how to deal with patient intolerance to nicotinamide and carbogen. Radiotherapy and oncology : journal of the European Society for Therapeutic Radiology and Oncology. 2004;70:301-9.

25. Troost EG, Laverman P, Kaanders JH, Philippens M, Lok J, Oyen WJ, et al. Imaging hypoxia after oxygenation-modification: comparing [18F]FMISO autoradiography with pimonidazole immunohistochemistry in human xenograft tumors. Radiother Oncol. 2006;80:157-64.

26. Hou H, Dong R, Lariviere JP, Mupparaju SP, Swartz HM, Khan N. Synergistic combination of hyperoxygenation and radiotherapy by repeated assessments of tumor pO2 with EPR oximetry. J Radiat Res. 2011;52:568-74.

27. Khan N, Li H, Hou H, Lariviere JP, Gladstone DJ, Demidenko E, et al. Tissue pO2 of orthotopic 9L and C6 gliomas and tumor-specific response to radiotherapy and hyperoxygenation. Int J Radiat Oncol Biol Phys. 2009;73:878-85.

28. Martin L, Lartigau E, Weeger P, Lambin P, Le Ridant AM, Lusinchi A, et al. Changes in the oxygenation of head and neck tumors during carbogen breathing. Radiother Oncol. 1993;27:123-30.

29. Janssens GO, Rademakers SE, Terhaard CH, Doornaert PA, Bijl HP, van den Ende P, et al. Accelerated radiotherapy with carbogen and nicotinamide for laryngeal cancer: results of a phase III randomized trial. J Clin Oncol. 2012;30:1777-83.

30. Schuuring J, Bussink J, Bernsen HJ, Peeters W, van der Kogel AJ. Effect of carbogen breathing on the radiation response of a human glioblastoma xenograft: analysis of 
186 |Chapter 9

hypoxia and vascular parameters of regrowing tumors. Strahlenther Onkol. 2006;182:408-14.

31. Bentzen SM, Gregoire V. Molecular imaging-based dose painting: a novel paradigm for radiation therapy prescription. Semin Radiat Oncol. 2011;21:101-10.

32. Lambin P, van Stiphout RG, Starmans MH, Rios-Velazquez E, Nalbantov G, Aerts HJ, et al. Predicting outcomes in radiation oncology--multifactorial decision support systems. Nat Rev Clin Oncol. 2013;10:27-40. 
DISCUSSION 



\section{CHAPTER}
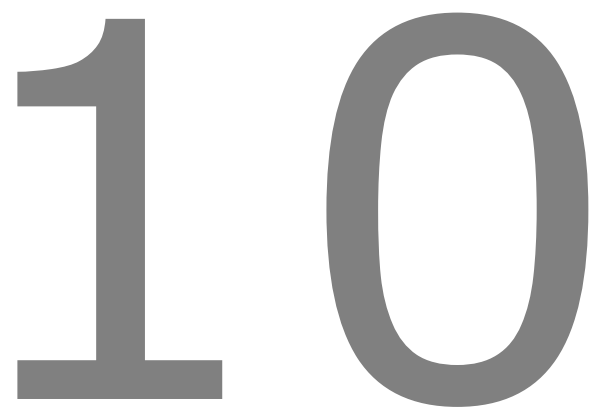

General discussion and summary 


\section{GENERAL DISCUSSION AND SUMMARY}

Tumor cell hypoxia is known to be a major factor that negatively influences treatment effectiveness, it promotes resistance to surgery, radiotherapy and chemotherapy and increases tumor aggressiveness, angiogenesis, and metastatic potential, resulting in a poor prognosis $(1,2)$. Tumor hypoxia is present in the majority of solid lesions. Detection and quantification of tumor hypoxia could help selecting patients who may benefit from hypoxia targeting treatment $(3,4)$. Non-invasive positron emission tomography (PET) imaging techniques provide the opportunity to perform repeated tumor hypoxia measurements, additionally it allows visualization of the spatial distribution of hypoxia $(5,6)$.

Several 2-nitroimidazoles, labeled with fluor-18 $\left[{ }^{18} \mathrm{~F}\right]$, have already been applied to identify hypoxia $(5,7)$. The selective binding and retention of 2-nitroimidazoles allows detection and quantification of tumor hypoxia prior to and during treatment (8-10). An extensive literature overview of hypoxia PET imaging, using 2-nitroimadazoles is given in chapter 2. The preclinical and clinical use of hypoxia PET imaging is described, with a focus on its validation, quantification and (clinical) applications. From this study we observed that several 2-nitroimidazole-based PET tracers have the ability to reliably measure tumor hypoxia, that these tracers have a prognostic value for treatment outcome in several cancers and that it is feasible to use hypoxia PET imaging to select patients for additional anti-hypoxia treatment.

\section{Comparison of hypoxia PET tracers}

The diversity in the available hypoxia PET tracers raises the question which tracer is the most optimal. In the available literature a large variety of different tracers, tumor models or patient populations were used, in addition, the scan time points and image analysis varied $(5,11)$. Therefore, there is a need for comparative studies, investigating different hypoxia PET tracers within one tumor model using the same acquisition protocol and analysis parameters. We performed an extensive comparison of the frequently used hypoxia PET tracers $\left[{ }^{18} \mathrm{~F}\right] \mathrm{HX} 4,\left[{ }^{18} \mathrm{~F}\right] \mathrm{FAZA}$ and $\left[{ }^{18} \mathrm{~F}\right] \mathrm{FMISO}$ within one preclinical (rat rhabdomyosarcoma) tumor model. This tumor model was previously characterized and used for hypoxia PET imaging with $\left[{ }^{18} \mathrm{~F}\right] \mathrm{FMISO},\left[{ }^{18} \mathrm{~F}\right] \mathrm{EF} 3$ and $\left[{ }^{18} \mathrm{~F}\right] \mathrm{HX} 4$ and clinical relevant fractions of hypoxia were reported (12-14). The direct comparison of the tracers, described in chapter 3, provides more insight into the strengths and weaknesses of the hypoxia PET tracers. We observed that $\left[{ }^{18} \mathrm{~F}\right] \mathrm{HX} 4$ provides a significantly higher tumor-to-background ratio in comparison to $\left[{ }^{18} \mathrm{~F}\right] \mathrm{FAZA}$ or $\left[{ }^{18} \mathrm{~F}\right] \mathrm{FMISO}$ at the same image time point. These results are in agreement with the clinical observation of Chen et al. which showed that $\left[{ }^{18} \mathrm{~F}\right] \mathrm{HX} 4$ at $1.5 \mathrm{~h}$ p.i. reached the same contrast than $\left[{ }^{18} \mathrm{~F}\right] \mathrm{FMISO}$ at $2 \mathrm{~h}$ p.i. (15). Carlin et al. (16), on the other hand, reported no statistical difference in the tumor-to-muscle ratios of the hypoxia PET tracers $\left[{ }^{18} \mathrm{~F}\right] \mathrm{HX} 4,\left[{ }^{18} \mathrm{~F}\right] \mathrm{FAZA}$ and $\left[{ }^{18} \mathrm{~F}\right] \mathrm{FMISO}$ in mice bearing SQ2Ob xenograft tumors. This difference might be explained by the low number of tumors (3-5 per tracer) investigated by Carlin et al. and the short injection-acquisition time interval. In chapter 3 we also observed a high repeatability of the $\left[{ }^{18} \mathrm{~F}\right] \mathrm{HX} 4$ and $\left[{ }^{18} \mathrm{~F}\right] \mathrm{FMISO}$ uptake in a testretest (48h interval) setting, while for $\left[{ }^{18} \mathrm{~F}\right] \mathrm{FAZA}$ lower spatial reproducibility was observed. However, in mice bearing a human SiHa cervix tumor xenograft $\left[{ }^{18}\right.$ F]FAZA PET 
imaging showed a high spatial reproducibility (17). From the current literature and our analysis in chapter 3 we can conclude that each tracer has its own strengths. Nevertheless, in clinical practice a high image contract within a short time interval is preferred, since the patient has to wait between injection of the radioactive tracer and the scan time p.i. Also, a good (spatial) reproducibility is necessary to allow additional anti-hypoxia treatment based on hypoxia PET imaging.

\section{Repeatability $\left[{ }^{18} \mathrm{~F}\right] \mathrm{HX} 4$ PET}

The ultimate aim of anti-cancer therapy is to improve the outcome of patients. We believe that targeting of tumor hypoxia with the aid of hypoxic cell sensitizers or a higher radiotherapy dose to the hypoxic regions could contribute to reach this goal. To select patients for additional anti-hypoxia therapy and to monitor the response to treatment, it is important to gain insight in the clinical day to day variability of the hypoxia PET uptake and the spatial location of the high uptake volumes. To answer this research question we performed image analysis on repeated $\left[{ }^{18} \mathrm{~F}\right] \mathrm{HX} 4$ PET data, acquired within a time interval of 1 to 6 days, from a multi-centered study (NCT01075399) in patients with head and neck and lung cancer (Chapter 4). The results of this study showed a high repeatability of the $\left[{ }^{18} \mathrm{~F}\right] \mathrm{HX} 4$ PET uptake parameters $\left(\mathrm{SUV}_{\max }, \mathrm{SUV}_{\text {mean, }}\right.$ TBR, hypoxic volume), with for example a repeatability percentage of $17 \%$ and $15 \%$ for $S U V_{\max }$ and $S U V_{\text {mean }}$, respectively. These percentages are much lower than the repeatability expected based on $\left[{ }^{18}\right.$ F]FDG PET images, which would be approximately $35 \%$ in the SUV range (18). A previous study with $\left[{ }^{18} \mathrm{~F}\right] \mathrm{FMISO}$ in eleven patients with head and neck cancer reported similar results, showing a high reproducibility of the $\left[{ }^{18}\right.$ F]FMISO PET parameters SUV ${ }_{\max }$, TBR and hypoxic volume (19). The observed repeatability of the $\left[{ }^{18} \mathrm{~F}\right] \mathrm{HX} 4$ PET parameters provides evidence for a reliable detection and quantification of tumor hypoxia. $\left[{ }^{18} \mathrm{~F}\right] \mathrm{HX} 4 \mathrm{PET}$ parameters on a global tumor level might therefore be able to monitor the response to treatment or guide the identification of patients who might benefit from additional anti-hypoxia treatment (4). However, for radiotherapy dose escalation also the repeatability of the PET uptake on a sub-volume or voxel level is important, since this will provide information on the spatial distribution of tumor hypoxia and the possibility to target these radio-resistant subvolumes with a higher radiation dose $(5,20)$. In the patient population studied in chapter 4 , we observed also a high spatial repeatability in the majority of patients. These results confirmed the observation in the preclinical study (chapter 3). However, previous repeatability studies using the alternative hypoxia tracers $\left[{ }^{18} \mathrm{~F}\right] \mathrm{FMISO}$ or $\left[{ }^{18} \mathrm{~F}\right] \mathrm{FAZA}$ reported contradictory results regarding the spatial uptake pattern of the hypoxia PET tracers (10, $17,19,21)$. Nevertheless, our current results show that $\left[{ }^{18} \mathrm{~F}\right] \mathrm{HX} 4$ PET can reliably identify the hypoxic volumes before the start of treatment, which might benefit from treatment with an increased radiation dose.

\section{Optimal imaging}

The beneficial properties of $\left[{ }^{18} \mathrm{~F}\right] \mathrm{HX} 4$, observed in the preclinical tumor model (chapter 3 ), the promising results of the phase I clinical trial (22) and the observed clinical repeatability (chapter 4) provide a good basis for phase II clinical trials. $\left[{ }^{18} \mathrm{~F}\right] \mathrm{HX} 4$ PET imaging was incorporated as a translational research part in two clinical studies at Maastro Clinic 
(NCT01024829 \& NCT01210378) and additionally a new imaging study with $\left[{ }^{18} \mathrm{~F}\right] \mathrm{HX} 4$ in patients with HNSCC was initiated (NCT01347281). As mentioned previously, the available hypoxia PET data in the literature is diverse and acquisition protocols frequently vary, which makes it hard to compare and combine the published hypoxia PET studies $(5,11)$. Our aim was to standardize acquisition and image analysis in an early stage. Therefore we defined in the first 15 non-small cell lung cancer (NSCLC) patients the optimal image parameters for $\left[{ }^{18} \mathrm{~F}\right] \mathrm{HX} 4 \mathrm{PET} / \mathrm{CT}$ imaging (chapter 5 ). We observed heterogeneous but stable uptake patterns between PET images acquired at $2 \mathrm{~h}$ or $4 \mathrm{~h}$ p.i., indicating a high short-time reproducibility of the PET uptake. However, at $4 \mathrm{~h}$ p.i. imaging contrast (tumorbackground ratio) was superior to $2 \mathrm{~h}$ p.i. These results were confirmed in the first 10 patients with a HNSCC, where the image contrast increased significantly up to $4 \mathrm{~h}$ p.i. (chapter 8 ). In addition, preliminary results of another research group, investigating the use of $\left[{ }^{18} \mathrm{~F}\right] \mathrm{HX} 4 \mathrm{PET}$ in patients with pancreatic and esophageal cancer report similar observations (AMC Amsterdam, NCT01995084, (23)).

Unfortunately, it was clinically not feasible to perform imaging beyond $4 \mathrm{~h}$ p.i. and no information was acquired regarding the clinical $\left[{ }^{18} \mathrm{~F}\right] \mathrm{HX} 4$ PET image contrast at later time points. In preclinical setting the contrast in the images remained stable from $4 \mathrm{~h}$ to $6 \mathrm{~h}$ p.i. (12). However, comparing the preclinical and clinical results in this thesis, we observed that the estimated half life of $\left.{ }^{18} \mathrm{~F}\right] \mathrm{HX} 4$ (clearance from blood) was 2.2 hours for the rat rhabdomyosarcoma study (chapter 3 ), while this was 4.3 hours for the first NSCLC patients described in chapter 5 . Therefore, a later acquisition time point might be beneficial in patients. Nevertheless, the continuing decay of the radionuclide would cause a decrease of the signal to noise ratio, requiring the injection of a higher tracer dose or a longer acquisition time. These technical aspects in combination with the practical disadvantages supports the decision not to increase the time interval between the $\left[{ }^{18} \mathrm{~F}\right] \mathrm{HX} 4$ injection and PET scan in clinical setting.

\section{$\left[{ }^{18} \mathrm{~F}\right] \mathrm{HX} 4$ versus $\left[{ }^{18} \mathrm{~F}\right]$ FDG PET}

Hypoxia PET imaging is still in the research stage, and is not used in routine clinical practice. $\left[{ }^{18}\right.$ F]FDG PET, on the other hand, is a frequently used tracer to visualize tumor metabolism and may indirectly reflect the tumor microenvironment, including areas of hypoxia $(1,24,25)$. We defined the hypoxic and metabolic status of NSCLC (chapter 6 ) and HNSCC (chapter 7), with the aid of $\left[{ }^{18} \mathrm{~F}\right] \mathrm{HX} 4$ and $\left[{ }^{18} \mathrm{~F}\right] \mathrm{FDG}$ PET imaging. In both studies we observed a correlation between the volume of the lesion and the overall $\left[{ }^{18} \mathrm{~F}\right] \mathrm{HX} 4$ and $\left[{ }^{18} \mathrm{~F}\right]$ FDG PET uptake. This was in agreement with previous studies investigating the correlation between $\left[{ }^{18} \mathrm{~F}\right] \mathrm{FMISO}$ PET and $\left[{ }^{18} \mathrm{~F}\right] \mathrm{FDG}$ PET parameters in patients with head and neck cancer (26-28). However, for patients with NSCLC contradictory results were reported in literature (29-31). General correlations on a tumor level, however, provide no information about the spatial orientation of a high metabolism or hypoxia. Therefore additional analysis on a sub-volume level were performed evaluating the agreement between volumes with a high metabolism (uptake $>50 \%$ SUV $_{\max }$ ) and tumor hypoxia $(T B R>1.4$ ). Note that the thresholds to define a high metabolism and hypoxia were defined arbitrarily, based on ongoing $\left[{ }^{18} \mathrm{~F}\right] \mathrm{FDG}$ boost trials $(32,33)$, and previous publications on hypoxia PET imaging $(12,13,34,35)$. A change in the definition will result in different high uptake volumes and results. In radiotherapy dose escalation, pre-defined thresholds are only 
relevant if a 'dose painting by contour' approach is selected, treating the high uptake volumes with a higher dose level. For 'dose painting by numbers', on the other hand, thresholds do not play a role, since the dose is based on the local voxel intensity values within the tumor (36).

Nevertheless, based on our predefined threshold to define high metabolism and hypoxia a (partial) mismatch was observed in approximately $50 \%$ and $70 \%$ of the primary NSCLC and HNSCC lesions, respectively (chapter 6 and 7). The fraction of the hypoxic volume, located outside the high $\left[{ }^{18} \mathrm{~F}\right]$ FDG volume, was on average similar for NSCLC (24\%) and HNSCC (25\%). However, only in the NSCLC patients a distinct uptake pattern between the high $\left[{ }^{18} \mathrm{~F}\right] \mathrm{HX} 4$ and $\left[{ }^{18} \mathrm{~F}\right] \mathrm{FDG}$ uptake volumes was observed in 2 cases. This can be explained by the patient populations used in the studies. The NSCLC population consists of tumors with a heterogeneous pathology, including mainly patients with an adenocarcinoma of the lung, while the head and neck cancer patients all suffered from a squamous cell carcinoma. In the literature it is described that adeno and squamous cell carcinomas show differences in their metabolism. Where adenocarcinomas rely mainly on aerobic glycolysis, squamous cell carcinomas have a more physiologically metabolism, using mitochondrial oxidation with anaerobic glycolysis under hypoxia (37). Although we observed in both patient populations a partial agreement between the extend and location of hypoxia and metabolism, they represent different properties of the tumor and provide complementary information which can be used as a prognostic marker for outcome $(2,9,38-40)$, to select patients for additional therapy $(4,41,42)$ or to target the resistant (high metabolic or hypoxic) volumes within the tumor $(32,33,43,44)$.

\section{Monitoring response}

The ability to monitor the response to treatment could provide valuable information to adapt the treatment in an early stage. The results of previous studies showed that hypoxia PET imaging with $\left[{ }^{18} \mathrm{~F}\right] \mathrm{FMISO}$ and metabolic $\left[{ }^{18} \mathrm{~F}\right] \mathrm{FDG}$ PET imaging were able to observe changes in the uptake during (chemo)radiotherapy, and that these changes had a higher predictive value for the treatment outcome than pre-treatment measurements $(9,39,45$, 46). We performed a study to investigate the treatment-associated changes in tumor hypoxia using $\left[{ }^{18} \mathrm{~F}\right] \mathrm{HX} 4 \mathrm{PET}$ imaging and hypoxia-related blood biomarkers (chapter 8). In agreement with previous literature, a significant decrease of the hypoxia $\left.{ }^{18} \mathrm{~F}\right] \mathrm{HX} 4$ PET uptake was observed during treatment with radiotherapy alone or in combination with cisplatin or cetuximab. The evaluated blood biomarkers, on the other hand, were not specific enough to measure a significant decrease in tumor hypoxia during treatment. This might be caused by the included patient cohort, since the observed plasma levels of plasma osteopontin and CAIX were relative low in comparison to previous studies, reporting the osteopontin and CAIX levels in large numbers of head and neck cancer and rectal cancer patients $(42,47)$. In addition, plasma osteopontin is also known to play a role in the immune regulation and stress response which might also be induced by radiation (48). In our patient cohort, and based on our definition of tumor hypoxia (TBR>1.4), only 2 lesions had a remaining hypoxic volume $\left(>1 \mathrm{~cm}^{3}\right)$ during treatment. For these lesions, the localization of the hypoxic volume was (almost) completely within the hypoxic volume defined at baseline, which suggests that the localisation of persistent hypoxia is stable during treatment. This would allow the boosting of hypoxic subvolumes defined at the start of treat- 
ment. However, previous studies using alternative hypoxia PET tracers showed contradictory results, reporting a stable localisation (10) or a spatial move during treatment $(21,49$, 50). All studies show results of a relative low number of patients, and should be interpreted with care. Nevertheless, potential changes in the spatial distribution of hypoxia should be taken into consideration when applying hypoxia-guided dose escalation (49-51).

\section{Targeting of tumor hypoxia}

Tumor hypoxia can be targeted with the aid of hypoxia-activated prodrugs, which are activated in an environment with a low oxygen concentration. TH302 is a one of these drugs, which is activated on the reduction of its 2-nitroimidazole component, eventually releasing the active drug bromo-isophosphoramide mustard that acts as a DNA crosslinker which leads to cell death. Several clinical Phase I and II studies have been performed, investigating the safety and the potential of TH302 in combination with chemotherapy, with promising results (52-55). Since TH302 specifically targets the hypoxic cells, which are more resistant for radiation, the combination of radiotherapy with TH302 might have also the potential to improve tumor control. In chapter 9 we studied in a preclinical setting, for the first time, the effect of radiotherapy in combination with the anti-hypoxia drug TH302. [ $\left.{ }^{18} \mathrm{~F}\right] \mathrm{HX} 4$ PET was used to evaluate the response to treatment. In addition the relationship between hypoxia PET imaging and the therapeutic outcome was assessed. We observed that the efficiency of the treatment was dependent on the oxygenation status, where a decreased oxygenation status increased the therapeutic effect of TH302. The pretreatment hypoxic fraction was associated with tumor growth delay (time to reach $4 \mathrm{x}$ startvolume) and $\left[{ }^{18} \mathrm{~F}\right] \mathrm{HX} 4$ PET imaging was able to detect a decrease in the hypoxic fraction after treatment with TH302. These results confirm that hypoxia PET imaging could be used to select patients with a hypoxic tumor, which will benefit the most from the additional treatment with $\mathrm{TH} 302$. In addition, serial imaging of tumor hypoxia could be useful to assess the effect of TH302. These promising results will be translated to a clinical trial in patients with esophageal cancer, with the aim to assess the maximum tolerated dose and anti-tumor activity of $\mathrm{TH} 302$ in combination with chemoradiation. In addition the prognostic value of $\left[{ }^{18} \mathrm{~F}\right] \mathrm{HX} 4$ PET imaging at baseline and after administration of $\mathrm{TH} 302$ will be explored. 


\section{REFERENCES}

1. Harris AL. Hypoxia-a key regulatory factor in tumour growth. Nat Rev Cancer. 2002;2:38-47.

2. Lambin P, van Stiphout RG, Starmans MH, Rios-Velazquez E, Nalbantov G, Aerts HJ, et al. Predicting outcomes in radiation oncology--multifactorial decision support systems. Nat Rev Clin Oncol. 2013;10:27-40.

3. Overgaard J. Hypoxic modification of radiotherapy in squamous cell carcinoma of the head and neck - A systematic review and meta-analysis. Radiother Oncol. 2011;100:22-32.

4. Wilson WR, Hay MP. Targeting hypoxia in cancer therapy. Nat Rev Cancer. 2011;11:393-410.

5. Horsman MR, Mortensen LS, Petersen JB, Busk M, Overgaard J. Imaging hypoxia to improve radiotherapy outcome. Nat Rev Clin Oncol. 2012;9:674-87.

6. Bussink J, van Herpen CM, Kaanders JH, Oyen WJ. PET-CT for response assessment and treatment adaptation in head and neck cancer. Lancet Oncol. 2010;11:661-9.

7. Lopci E, Grassi I, Chiti A, Nanni C, Cicoria G, Toschi L, et al. PET radiopharmaceuticals for imaging of tumor hypoxia: a review of the evidence. Am J Nucl Med Mol Imaging. 2014;4:365-84.

8. Bollineni VR, Wiegman EM, Pruim J, Groen HJ, Langendijk JA. Hypoxia imaging using Positron Emission Tomography in non-small cell lung cancer: implications for radiotherapy. Cancer Treat Rev. 2012;38:1027-32.

9. Zips D, Zophel K, Abolmaali N, Perrin R, Abramyuk A, Haase R, et al. Exploratory prospective trial of hypoxia-specific $\mathrm{PET}$ imaging during radiochemotherapy in patients with locally advanced head-and-neck cancer. Radiother Oncol. 2012;105:21-8.

10. Bittner MI, Wiedenmann N, Bucher S, Hentschel M, Mix M, Weber WA, et al. Exploratory geographical analysis of hypoxic subvolumes using 18F-MISO-PET imaging in patients with head and neck cancer in the course of primary chemoradiotherapy. Radiother Oncol. 2013;108:511-6.

11. Thorwarth D, Monnich D, Zips D. Methodological aspects on hypoxia PET acquisition and image processing. Q J Nucl Med Mol Imaging. 2013;57:235-43.

12. Dubois LJ, Lieuwes NG, Janssen MH, Peeters WJ, Windhorst AD, Walsh JC, et al. Preclinical evaluation and validation of [18F]HX4, a promising hypoxia marker for PET imaging. Proc Natl Acad Sci U S A. 2011;108:14620-5.

13. Dubois L, Landuyt W, Haustermans K, Dupont $P$, Bormans $G$, Vermaelen $P$, et al. Evaluation of hypoxia in an experimental rat tumour model by [(18)F] fluoromisonidazole PET and immunohistochemistry. Br J Cancer. 2004;91:1947-54.

14. Dubois L, Landuyt W, Cloetens L, Bol A, Bormans G, Haustermans K, et al. [18F]EF3 is not superior to [18F]FMISO for PET-based hypoxia evaluation as measured in a rat rhabdomyosarcoma tumour model. Eur J Nucl Med Mol Imaging. 2009;36:209-18.

15. Chen L, Zhang Z, Kolb HC, Walsh JC, Zhang J, Guan Y. (1)(8)F-HX4 hypoxia imaging with PET/CT in head and neck cancer: a comparison with (1)(8)F-FMISO. Nucl Med Commun. 2012;33:1096-102. 
16. Carlin S, Zhang H, Reese M, Ramos NN, Chen Q, Ricketts SA. A comparison of the imaging characteristics and microregional distribution of 4 hypoxia PET tracers. J Nucl Med. 2014;55:515-21.

17. Busk M, Mortensen LS, Nordsmark M, Overgaard J, Jakobsen S, Hansen KV, et al. PET hypoxia imaging with FAZA: reproducibility at baseline and during fractionated radiotherapy in tumour-bearing mice. Eur J Nucl Med Mol Imaging. 2013;40:186-97.

18. de Langen AJ, Vincent A, Velasquez LM, van Tinteren H, Boellaard R, Shankar LK, et al. Repeatability of 18F-FDG uptake measurements in tumors: a metaanalysis. J Nucl Med. 2012;53:701-8.

19. Okamoto S, Shiga T, Yasuda K, Ito YM, Magota K, Kasai K, et al. High reproducibility of tumor hypoxia evaluated by $18 \mathrm{~F}$-fluoromisonidazole PET for head and neck cancer. J Nucl Med. 2013;54:201-7.

20. Grootjans W, de Geus-Oei LF, Troost EG, Visser EP, Oyen WJ, Bussink J. PET in the management of locally advanced and metastatic NSCLC. Nat Rev Clin Oncol. 2015.

21. Nehmeh SA, Lee NY, Schroder H, Squire O, Zanzonico PB, Erdi YE, et al. Reproducibility of intratumor distribution of (18)F-fluoromisonidazole in head and neck cancer. Int J Radiat Oncol Biol Phys. 2008;70:235-42.

22. van Loon J, Janssen MH, Ollers M, Aerts HJ, Dubois L, Hochstenbag M, et al. PET imaging of hypoxia using [18F]HX4: a phase I trial. Eur J Nucl Med Mol Imaging. 2010;37:1663-8.

23. Klaassen R, van Laarhoven $H$, Tienhoven $G$, Bijlsma M, Geijsen E, Besselink M, et al. Timing and reproducibility of [18F]HX4 PET-CT for the detection of hypoxia in esophageal and pancreatic cancer Journal of nuclear medicine; May 2014 vol 55, abstract 14332014.

24. Gatenby RA, Gillies RJ. Why do cancers have high aerobic glycolysis? Nat Rev Cancer. 2004;4:891-9.

25. Dierckx RA, Van de Wiele C. FDG uptake, a surrogate of tumour hypoxia? Eur J Nucl Med Mol Imaging. 2008;35:1544-9.

26. Rajendran JG, Mankoff DA, O'Sullivan F, Peterson LM, Schwartz DL, Conrad EU, et al. Hypoxia and glucose metabolism in malignant tumors: evaluation by [18F]fluoromisonidazole and [18F]fluorodeoxyglucose positron emission tomography imaging. Clin Cancer Res. 2004;10:2245-52.

27. Zimny M, Gagel B, DiMartino E, Hamacher K, Coenen HH, Westhofen M, et al. FDG--a marker of tumour hypoxia? A comparison with [18F]fluoromisonidazole and pO2polarography in metastatic head and neck cancer. Eur J Nucl Med Mol Imaging. 2006;33:1426-31.

28. Sato J, Kitagawa $Y$, Yamazaki $Y$, Hata H, Okamoto S, Shiga $T$, et al. 18Ffluoromisonidazole PET uptake is correlated with hypoxia-inducible factor-1alpha expression in oral squamous cell carcinoma. J Nucl Med. 2013;54:1060-5.

29. Bollineni VR, Kerner GS, Pruim J, Steenbakkers RJ, Wiegman EM, Koole MJ, et al. PET Imaging of Tumor Hypoxia Using 18F-Fluoroazomycin Arabinoside in Stage III-IV NonSmall Cell Lung Cancer Patients. J Nucl Med. 2013.

30. Cherk MH, Foo SS, Poon AM, Knight SR, Murone C, Papenfuss AT, et al. Lack of correlation of hypoxic cell fraction and angiogenesis with glucose metabolic rate in non-small cell lung cancer assessed by 18 F-Fluoromisonidazole and 18F-FDG PET. J Nucl Med. 2006;47:1921-6. 
31. Vera P, Bohn P, Edet-Sanson A, Salles A, Hapdey S, Gardin I, et al. Simultaneous positron emission tomography (PET) assessment of metabolism with (1)(8)F-fluoro-2deoxy-d-glucose (FDG), proliferation with (1)(8)F-fluoro-thymidine (FLT), and hypoxia with (1)(8)fluoro-misonidazole (F-miso) before and during radiotherapy in patients with non-small-cell lung cancer (NSCLC): a pilot study. Radiother Oncol. 2011;98:109-16.

32. van Elmpt W, De Ruysscher D, van der Salm A, Lakeman A, van der Stoep J, Emans D, et al. The PET-boost randomised phase II dose-escalation trial in non-small cell lung cancer. Radiother Oncol. 2012;104:67-71.

33. Heukelom J, Hamming O, Bartelink H, Hoebers F, Giralt J, Herlestam T, et al. Adaptive and innovative Radiation Treatment FOR improving Cancer treatment outcomE (ARTFORCE); a randomized controlled phase II trial for individualized treatment of head and neck cancer. BMC Cancer. 2013;13:84.

34. Zegers $\mathrm{CM}$, van Elmpt W, Wierts R, Reymen B, Sharifi H, Ollers MC, et al. Hypoxia imaging with [(18)F]HX4 PET in NSCLC patients: Defining optimal imaging parameters. Radiother Oncol. 2013;109:58-64.

35. Rasey JS, Koh WJ, Evans ML, Peterson LM, Lewellen TK, Graham MM, et al. Quantifying regional hypoxia in human tumors with positron emission tomography of [18F]fluoromisonidazole: a pretherapy study of 37 patients. Int J Radiat Oncol Biol Phys. 1996;36:417-28.

36. Thorwarth $D$, Geets $X$, Paiusco $M$. Physical radiotherapy treatment planning based on functional PET/CT data. Radiother Oncol. 2010;96:317-24.

37. Meijer TW, Schuurbiers OC, Kaanders JH, Looijen-Salamon MG, de Geus-Oei LF, Verhagen AF, et al. Differences in metabolism between adeno- and squamous cell non-small cell lung carcinomas: spatial distribution and prognostic value of GLUT1 and MCT4. Lung Cancer. 2012;76:316-23.

38. Thorwarth D, Eschmann SM, Holzner F, Paulsen F, Alber M. Combined uptake of [18F]FDG and [18F]FMISO correlates with radiation therapy outcome in head-andneck cancer patients. Radiother Oncol. 2006;80:151-6.

39. van Elmpt $W$, Ollers $M$, Dingemans $A M$, Lambin $P$, De Ruysscher $D$. Response assessment using 18F-FDG PET early in the course of radiotherapy correlates with survival in advanced-stage non-small cell lung cancer. J Nucl Med. 2012;53:1514-20.

40. Sato J, Kitagawa Y, Yamazaki Y, Hata H, Asaka T, Miyakoshi M, et al. Advantage of FMISO-PET over FDG-PET for predicting histological response to preoperative chemotherapy in patients with oral squamous cell carcinoma. Eur J Nucl Med Mol Imaging. 2014;41:2031-41.

41. Rischin D, Hicks RJ, Fisher R, Binns D, Corry J, Porceddu S, et al. Prognostic significance of [18F]-misonidazole positron emission tomography-detected tumor hypoxia in patients with advanced head and neck cancer randomly assigned to chemoradiation with or without tirapazamine: a substudy of Trans-Tasman Radiation Oncology Group Study 98.02. J Clin Oncol. 2006;24:2098-104.

42. Overgaard J, Eriksen JG, Nordsmark M, Alsner J, Horsman MR. Plasma osteopontin, hypoxia, and response to the hypoxia sensitiser nimorazole in radiotherapy of head and neck cancer: results from the DAHANCA 5 randomised double-blind placebocontrolled trial. Lancet Oncol. 2005;6:757-64.

43. Thorwarth D, Eschmann SM, Paulsen F, Alber M. Hypoxia dose painting by numbers: a planning study. Int J Radiat Oncol Biol Phys. 2007;68:291-300. 
44. Toma-Dasu I, Uhrdin J, Antonovic L, Dasu A, Nuyts S, Dirix P, et al. Dose prescription and treatment planning based on FMISO-PET hypoxia. Acta Oncol. 2012;51:222-30.

45. Servagi-Vernat S, Differding S, Hanin FX, Labar D, Bol A, Lee JA, et al. A prospective clinical study of (1)(8)F-FAZA PET-CT hypoxia imaging in head and neck squamous cell carcinoma before and during radiation therapy. Eur J Nucl Med Mol Imaging. 2014;41:1544-52.

46. Bollineni VR, Koole MJ, Pruim J, Brouwer CL, Wiegman EM, Groen HJ, et al. Dynamics of tumor hypoxia assessed by 18F-FAZA PET/CT in head and neck and lung cancer patients during chemoradiation: possible implications for radiotherapy treatment planning strategies. Radiother Oncol. 2014;113:198-203.

47. Buijsen J, van Stiphout RG, Menheere PP, Lammering G, Lambin P. Blood biomarkers are helpful in the prediction of response to chemoradiation in rectal cancer: a prospective, hypothesis driven study on patients with locally advanced rectal cancer. Radiother Oncol. 2014;111:237-42.

48. Wang KX, Denhardt DT. Osteopontin: role in immune regulation and stress responses. Cytokine Growth Factor Rev. 2008;19:333-45.

49. Lin Z, Mechalakos J, Nehmeh S, Schoder H, Lee N, Humm J, et al. The influence of changes in tumor hypoxia on dose-painting treatment plans based on 18F-FMISO positron emission tomography. Int J Radiat Oncol Biol Phys. 2008;70:1219-28.

50. Servagi-Vernat S, Differding S, Hanin FX, Labar D, Bol A, Lee JA, et al. A prospective clinical study of F-FAZA PET-CT hypoxia imaging in head and neck squamous cell carcinoma before and during radiation therapy. European journal of nuclear medicine and molecular imaging. 2014.

51. Servagi-Vernat S, Differding S, Sterpin E, Hanin FX, Labar D, Bol A, et al. Hypoxiaguided adaptive radiation dose escalation in head and neck carcinoma: A planning study. Acta Oncol. 2015:1-9.

52. Weiss GJ, Infante JR, Chiorean EG, Borad MJ, Bendell JC, Molina JR, et al. Phase 1 study of the safety, tolerability, and pharmacokinetics of TH-302, a hypoxia-activated prodrug, in patients with advanced solid malignancies. Clin Cancer Res. 2011;17:2997-3004.

53. Borad MJ, Reddy SG, Bahary N, Uronis HE, Sigal D, Cohn AL, et al. Randomized Phase II Trial of Gemcitabine Plus TH-302 Versus Gemcitabine in Patients With Advanced Pancreatic Cancer. J Clin Oncol. 2015;33:1475-81.

54. Ganjoo KN, Cranmer LD, Butrynski JE, Rushing D, Adkins D, Okuno SH, et al. A phase I study of the safety and pharmacokinetics of the hypoxia-activated prodrug $\mathrm{TH}-302$ in combination with doxorubicin in patients with advanced soft tissue sarcoma. Oncology. 2011;80:50-6.

55. Chawla SP, Cranmer LD, Van Tine BA, Reed DR, Okuno SH, Butrynski JE, et al. Phase II study of the safety and antitumor activity of the hypoxia-activated prodrug $\mathrm{TH}-302$ in combination with doxorubicin in patients with advanced soft tissue sarcoma. J Clin Oncol. 2014;32:3299-306. 


\section{CHAPTER}
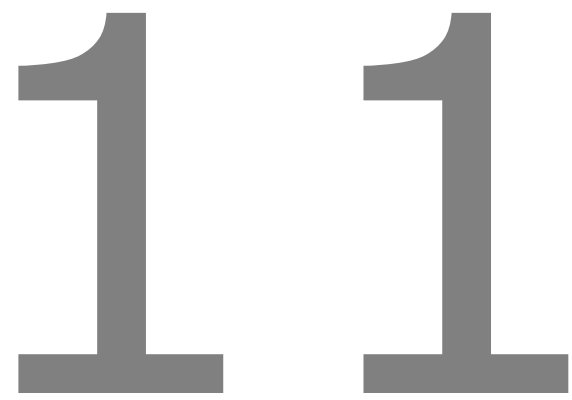

Concluding remarks and future perspectives 


\section{CONCLUDING REMARKS AND FUTURE PERSPECTIVES}

In this thesis we described hypoxia PET imaging with $\left[{ }^{18} \mathrm{~F}\right] \mathrm{HX} 4$ in preclinical setting, in patients with NSCLC and HNSCC. We showed its potential to reliably detect, visualize and quantify tumor hypoxia. Also, the additional value hypoxia PET imaging in comparison to metabolic FDG PET imaging was observed and we showed the ability to monitor the response to treatment with the aid of $\left[{ }^{18} \mathrm{~F}\right] \mathrm{HX} 4 \mathrm{PET}$.

\section{$\left[{ }^{18} \mathrm{~F}\right] \mathrm{HX} 4$ PET for other solid lesions.}

The focus of this thesis was the use of hypoxia PET imaging in patients with head and neck and lung cancer. However, hypoxia also plays an important role in other solid cancers and $\left[{ }^{18} \mathrm{~F}\right] \mathrm{HX} 4$ PET might have the potential to visualise and quantify hypoxia in more divers tumor types (1-6). For this reason we initiated clinical trials to investigate the potential of $\left.{ }^{[18} \mathrm{F}\right] \mathrm{HX} 4$ PET in patients with cervical cancer (NCT02233387), esophagus, prostate, glioblastoma, rectum or brain metastases (submitted to Medical Ethical Review Board). In addition, another research group recently completed a clinical trial investigating $\left[{ }^{18} \mathrm{~F}\right] \mathrm{HX} 4 \mathrm{PET}$ in patients with pancreatic and esophageal cancer (AMC Amsterdam, NCT01995084, (7)).

\section{Anti-hypoxia treatment}

A more individualized anti-cancer treatment could be obtained by the selection of patients for additional anti-hypoxia therapy. Studies including hypoxia PET imaging as part of the anti-hypoxia treatment intervention should be initiated, to stratify patients based on their hypoxic status. For example, treatment with accelerated radiotherapy in combination with carbogen inhalation and nicotinamide (ARCON; to decrease diffusion and perfusion limited hypoxia) was shown to result in additional benefit only in patients with a hypoxic tumor, which was assessed by pimonidazole staining (8). Also, the Phase II study of Rischin et al. (9) showed that the addition of the hypoxic cell cytotoxin tirapazamine to the standard chemoradiotherapy treatment was shown to be beneficial only for patients with a hypoxic tumor, which was identified by $\left[{ }^{18} \mathrm{~F}\right] \mathrm{FMISO}$ PET imaging. Previous results have also shown that the hypoxic status (based on plasma osteopontin concentration) had an important impact on the treatment effectiveness of the radiosensitizer nimorazole in combination with radiotherapy (10). This was confirmed in preclinical setting, where stratification of tumors, based ${ }^{18}$ F]FAZA PET imaging, showed only a significant improvement of the additional treatment with nimorazole in group with more tumor hypoxia (11). At MAASTRO Clinic we are currently investigating or initiating 'window-of-opportunity' trials. Using repeated hypoxia PET imaging before and after the therapeutic intervention, the effect of the drug on the hypoxic tumor status can be evaluated. One ongoing trial is evaluating the effect of the nitric oxide donor, nitroglycerin on the hypoxic status of NSCLC lesions. Our preliminary results show that nitroglycerin reduces the hypoxic fraction in patients with NSCLC (Figure 11.1, (12)). The effect of nitroglycerin in combination with radiotherapy on loco-regional control and the influence of the hypoxic status on the treatment effect are research questions that can be answered as soon as accrual and follow up has completed (NCT01210378). In addition, we will initiate a clinical trial, assessing the effect of $\mathrm{TH} 302$ in combination with chemoradiation. Also in this setting 
repeated hypoxia PET imaging will be performed, providing the opportunity to assess the hypoxic status before the start of treatment and evaluate the effect of TH302 on tumor hypoxia. In all studies using hypoxia interventions, the addition of hypoxia PET imaging will provide the opportunity to stratify patients based on their hypoxic status. Performing studies without the assessment of the hypoxic status, might give inconclusive results, since the treatment effect will be the average of patients with and without and hypoxic lesions $(8,10)$.
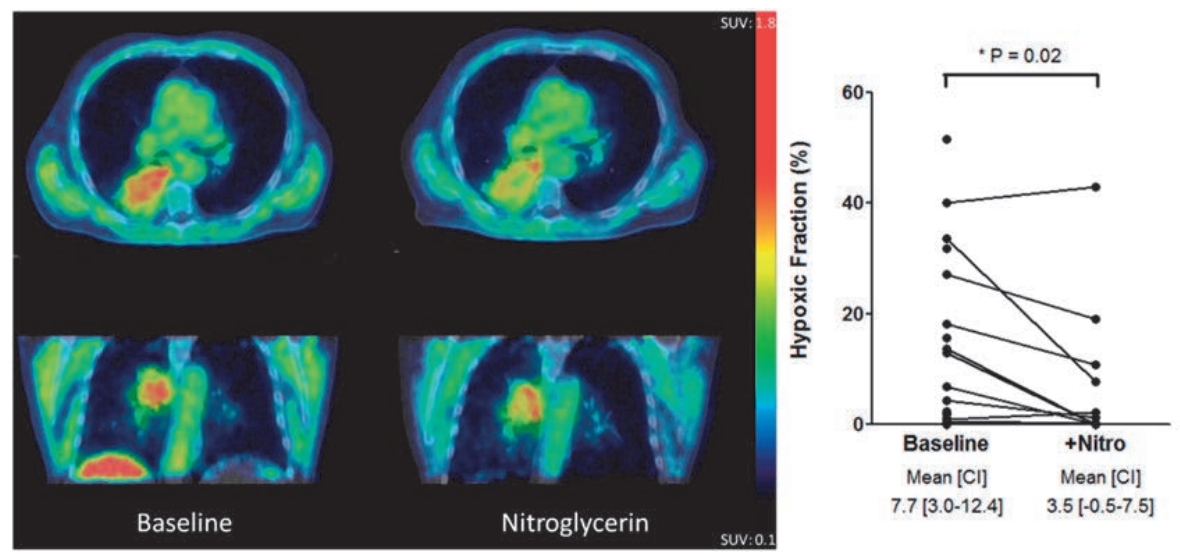

Figure 11.1: Effect of nitroglycerin on the hypoxic fraction (left) measured with $\left[{ }^{18} \mathrm{~F}\right] \mathrm{HX} 4 \mathrm{PET}$ ( $p$-value based on Wilcoxon signed rank test). A decrease in tumor uptake is clearly visible (right panel).

\section{Adaptation of the radiotherapy dose}

Currently, several studies are investigating the effect of a radiotherapy dose redistribution based on metabolic FDG PET imaging (13). The ongoing PET-Boost trial (NCT01024829, (14)) and ARTFORCE trial (NCT01504815, (15)) investigate the effect of higher radiation dose to the high FDG uptake volumes in patients with NSCLC and HNSCC patients, respectively. The results of this thesis, however, show that the volumes with a high $\left[{ }^{18} \mathrm{~F}\right] \mathrm{FDG}$ uptake do not always contain the total hypoxic volume. A boost to the high $\left[{ }^{18} \mathrm{~F}\right] \mathrm{FDG}$ volume could therefore miss part of the hypoxic sub-volume. The feasibility of a boost to the hypoxic regions was confirmed in several radiotherapy planning studies (16-20). Also a clinical trial is ongoing (University Hospital Tübingen, NCT02352792, (21)), investigating a hypoxia $\left(\left[{ }^{18} \mathrm{~F}\right] \mathrm{FMISO}\right)$ based radiotherapy dose escalation in combination with chemotherapy in patients with HNSCC. Nevertheless, conclusive information about the relationship between hypoxia, metabolism and the localization of recurrences after treatment is still lacking. There is a need for more information about the pattern of relapse and its association with hypoxia PET imaging. The current literature shows that recurrences are more likely to appear within the high $\left[{ }^{18} \mathrm{~F}\right] \mathrm{FDG}$ uptake volumes, defined before the start of treatment $(22,23)$. For hypoxia PET imaging, on the other hand, these results are limited. The study of Dirix et al. (24) showed for example in patients with a HNSCC, that all recurrences $(\mathrm{N}=9)$ were located within the high metabolic volume, however 3 of these recurrences appeared outside the hypoxic volume defined at baseline. As soon as the data of the patients included in this thesis have matured, a pattern of relapse analysis might give 
202 |Chapter 11

more information about the relationship between the localization of a high metabolism, hypoxia and loco-regional recurrences.

\section{Conclusion}

Imaging of tumor hypoxia with the aid of $\left[{ }^{18} \mathrm{~F}\right] \mathrm{HX} 4$ PET has the potential to contribute to individualized anti-cancer treatment by the accurate identification and quantification of tumor hypoxia and has the potential to monitor the response to treatment. Clinical studies investigating the use of hypoxia PET imaging to select patients for additional antihypoxia therapy, and an extensive pattern of relapse analysis are necessary to bring individualized treatment with the aid of hypoxia PET to the next level. 


\section{REFERENCES}

1. Carnell DM, Smith RE, Daley FM, Saunders MI, Bentzen SM, Hoskin PJ. An immunohistochemical assessment of hypoxia in prostate carcinoma using pimonidazole: implications for radioresistance. Int J Radiat Oncol Biol Phys. 2006;65:91-9.

2. Movsas B, Chapman JD, Hanlon AL, Horwitz EM, Greenberg RE, Stobbe C, et al. Hypoxic prostate/muscle pO2 ratio predicts for biochemical failure in patients with prostate cancer: preliminary findings. Urology. 2002;60:634-9.

3. Yue J, Yang Y, Cabrera AR, Sun X, Zhao S, Xie P, et al. Measuring tumor hypoxia with (18) F-FETNIM PET in esophageal squamous cell carcinoma: a pilot clinical study. Dis Esophagus. 2011.

4. Szeto MD, Chakraborty G, Hadley J, Rockne R, Muzi M, Alvord EC, Jr., et al. Quantitative metrics of net proliferation and invasion link biological aggressiveness assessed by MRI with hypoxia assessed by FMISO-PET in newly diagnosed glioblastomas. Cancer Res. 2009;69:4502-9.

5. Tanaka N, Kato H, Inose T, Kimura H, Faried A, Sohda M, et al. Expression of carbonic anhydrase 9, a potential intrinsic marker of hypoxia, is associated with poor prognosis in oesophageal squamous cell carcinoma. Br J Cancer. 2008;99:1468-75.

6. Vercellino L, Groheux D, Thoury A, Delord M, Schlageter MH, Delpech Y, et al. Hypoxia imaging of uterine cervix carcinoma with (18)F-FETNIM PET/CT. Clin Nucl Med. 2012;37:1065-8.

7. Klaassen R, van Laarhoven $H$, Tienhoven $G$, Bijlsma M, Geijsen E, Besselink M, et al. Timing and reproducibility of [18F]HX4 PET-CT for the detection of hypoxia in esophageal and pancreatic cancer Journal of nuclear medicine; May 2014 vol 55, abstract 14332014.

8. Janssens GO, Rademakers SE, Terhaard CH, Doornaert PA, Bijl HP, van den Ende P, et al. Accelerated radiotherapy with carbogen and nicotinamide for laryngeal cancer: results of a phase III randomized trial. J Clin Oncol. 2012;30:1777-83.

9. Rischin D, Hicks RJ, Fisher R, Binns D, Corry J, Porceddu S, et al. Prognostic significance of [18F]-misonidazole positron emission tomography-detected tumor hypoxia in patients with advanced head and neck cancer randomly assigned to chemoradiation with or without tirapazamine: a substudy of Trans-Tasman Radiation Oncology Group Study 98.02. J Clin Oncol. 2006;24:2098-104.

10. Overgaard J, Eriksen JG, Nordsmark M, Alsner J, Horsman MR. Plasma osteopontin, hypoxia, and response to the hypoxia sensitiser nimorazole in radiotherapy of head and neck cancer: results from the DAHANCA 5 randomised double-blind placebocontrolled trial. Lancet Oncol. 2005;6:757-64.

11. Tran LB, Bol A, Labar D, Cao-Pham TT, Jordan B, Gregoire V, et al. Predictive value of (18)F-FAZA PET imaging for guiding the association of radiotherapy with nimorazole: a preclinical study. Radiother Oncol. 2015;114:189-94.

12. Reymen B, Van Gisbergen M, Zegers CM, Dubois L, Lambin P. Nitroglycerin as a sensitizer in the treatment of non small cell lung cancer: from cells tin vitro to phase 3 trial. 3rd ESTRO Forum abstract SP-0597. 2015. 
13. Grootjans W, de Geus-Oei LF, Troost EG, Visser EP, Oyen WJ, Bussink J. PET in the management of locally advanced and metastatic NSCLC. Nat Rev Clin Oncol. 2015.

14. van Elmpt W, De Ruysscher D, van der Salm A, Lakeman A, van der Stoep J, Emans D, et al. The PET-boost randomised phase II dose-escalation trial in non-small cell lung cancer. Radiother Oncol. 2012;104:67-71.

15. Heukelom J, Hamming O, Bartelink H, Hoebers F, Giralt J, Herlestam T, et al. Adaptive and innovative Radiation Treatment FOR improving Cancer treatment outcomE (ARTFORCE); a randomized controlled phase II trial for individualized treatment of head and neck cancer. BMC Cancer. 2013;13:84.

16. Hendrickson K, Phillips M, Smith W, Peterson L, Krohn K, Rajendran J. Hypoxia imaging with [F-18] FMISO-PET in head and neck cancer: potential for guiding intensity modulated radiation therapy in overcoming hypoxia-induced treatment resistance. Radiother Oncol. 2011;101:369-75.

17. Toma-Dasu I, Uhrdin J, Antonovic L, Dasu A, Nuyts S, Dirix P, et al. Dose prescription and treatment planning based on FMISO-PET hypoxia. Acta Oncol. 2012;51:222-30.

18. Choi W, Lee SW, Park SH, Ryu JS, Oh SJ, Im KC, et al. Planning study for available dose of hypoxic tumor volume using fluorine-18-labeled fluoromisonidazole positron emission tomography for treatment of the head and neck cancer. Radiother Oncol. 2010;97:176-82.

19. Thorwarth D, Eschmann SM, Paulsen F, Alber M. Hypoxia dose painting by numbers: a planning study. Int J Radiat Oncol Biol Phys. 2007;68:291-300.

20. Servagi-Vernat S, Differding S, Sterpin E, Hanin FX, Labar D, Bol A, et al. Hypoxiaguided adaptive radiation dose escalation in head and neck carcinoma: A planning study. Acta Oncol. 2015:1-9.

21. Thorwarth D. Hypoxia PET imaging for delineation and response assessment during radiotherapy. 3rd ESTRO Forum abstract SP-0362. 2015.

22. Aerts HJ, van Baardwijk AA, Petit SF, Offermann C, Loon J, Houben R, et al. Identification of residual metabolic-active areas within individual NSCLC tumours using a pre-radiotherapy (18)Fluorodeoxyglucose-PET-CT scan. Radiother Oncol. 2009;91:386-92.

23. Due AK, Vogelius IR, Aznar MC, Bentzen SM, Berthelsen AK, Korreman SS, et al. Recurrences after intensity modulated radiotherapy for head and neck squamous cell carcinoma more likely to originate from regions with high baseline [18F]-FDG uptake. Radiother Oncol. 2014;111:360-5.

24. Dirix P, Vandecaveye V, De Keyzer F, Stroobants S, Hermans R, Nuyts S. Dose painting in radiotherapy for head and neck squamous cell carcinoma: value of repeated functional imaging with (18)F-FDG PET, (18)F-fluoromisonidazole PET, diffusionweighted MRI, and dynamic contrast-enhanced MRI. J Nucl Med. 2009;50:1020-7. 
VALORISATION ADDENDUM 



\section{VALORISATION ADDENDUM}

Tumor hypoxia is a property of cancer with a negative impact on the prognosis of the patient. In this thesis the possibilities to visualize, monitor and target tumor hypoxia are described. Since cancer is one of the leading causes of death in the Netherlands and its incidence is expected to rise due to an increased life expectancy of our elderly population, the scientific results of this thesis can be of benefit for the general society.

\section{Social relevance}

The treatment of cancer patients is continuously improving, with the aim to tailor cancer treatment to the needs of each individual patient. The presence of tumor hypoxia is known to have detrimental effects on the ability to control the disease. The non-invasive detection of tumor hypoxia provides the opportunity to individualize anti-cancer treatment by the ability (1) to improve the prediction of the response to treatment, (2) to monitor the response to treatment and (3) to select patients for additional anti-hypoxia treatment.

There are several prediction models available which estimate the chances of survival or local recurrences in cancer patients based on their general health and tumor characteristics. Several studies have shown that hypoxia is an important prognostic marker for treatment response. The addition of hypoxia PET parameters to already existing prediction models could improve the accuracy of these predictions. In the future these models can be used to intensify treatment in patients with a high chance of local recurrences.

The visualization of tumor hypoxia during treatment allows us to monitor the response to the given treatment and provides the ability to act on this information. This window-ofopportunity trial design might be very useful in the development of anti-hypoxia treatment strategies. The effect of hypoxia targeting by visualization before and after the treatment shows the effect of the (additional) treatment and its potential to target hypoxia. These studies are very effective, because with a limited amount of patients the effect of the treatment can be visualized, since each patient acts as its own control.

In addition, hypoxia imaging before treatment and the effect of anti-hypoxia treatment in the individual patients can be used for patient selection. Only patients with tumor hypoxia will have additional gain of anti-hypoxia treatment. Also the anti-hypoxia treatment should cause a decrease in hypoxia in the individual patient. Therefore patients that do not have significant hypoxia before the start of treatment, or patients that do not have a response to the additional treatment, could be excluded from unnecessary anti-hypoxia treatments.

Besides the clinical benefit, the ability to stratify patients based on their hypoxia status also provides economical benefit, since additional treatments to counteract hypoxia can be applied to only those patients who benefit from it, increasing their prognosis. Therefore, also unnecessary treatment, with additional costs and potential side-effects, can be omitted in the patients with no clinical benefit. 


\section{Target groups}

Patients and their doctors could benefit from the implementation of hypoxia imaging and targeting. An optimized and individualized treatment can be given, with less recurrences and a longer life expectancy. In addition, in the current health care environment patients are more and more involved in the treatment decisions. Improved prediction models and treatment monitoring will guide the doctors and patients in their treatment decisions.

Last, the opportunity to visualize tumor hypoxia can be of general interest to all health care companies which develop hypoxia targeted agents. The hypoxia PET tracers allow in an early stage to detect, in vivo, the effect of a hypoxia targeting drug, which can guide them in the development.

\section{Activities and products}

In this thesis the included number of patients is still limited. However, patient accrual is still ongoing in the described clinical trials and new clinical trials are initiated. As soon as sufficient data are gathered, the additional value of hypoxia PET imaging to the current prediction models can be evaluated. There are already several prediction models available (for example on www.predictcancer.org). Hypoxia PET imaging data could be integrated into these prediction models. The current data might provide additional information improving the response prediction.

At Maastro there is a research group investigating the potential of quantitative features from medical images (RADIOMICS) to monitor the response to treatment http://www.radiomics.org/. At the moment research is performed mainly on CT and $\left[{ }^{18}\right.$ F]FDG PET imaging. Hypoxia PET imaging could be integrated in this extensive image analysis, and analysis are planned for the near future.

\section{Innovation}

The detection of tumor hypoxia is not new. However the clinical use of hypoxia PET imaging to monitor or predict the response to treatment is limited. Also, treatment selection based on hypoxia PET imaging is not yet implemented. In this thesis we mainly describe the use of the hypoxia PET tracer HX4, this is a relative new PET tracer for hypoxia PET imaging. Comparing this tracer with the alternative hypoxia PET tracers on the market showed beneficial characteristics which motivated the use of this tracer in clinical trials. We showed the ability of HX4 PET imaging to monitor the response to radiotherapy, in patients with head and neck cancer, and the response to TH302 treatment in preclinical setting.

\section{Planning and realisation}

The research described in this thesis is the basis of several new initiatives to implement hypoxia PET imaging. The follow-up data of all patients described in this thesis will provide information on the ability to predict the response to treatment. This information on tumor hypoxia will be implemented in the available prediction models. To perform the research described in this thesis, a close collaboration with Threshold Pharmaceuticals was started. 
This led to new opportunities regarding clinical trials. The next step is a phase I trial investigating the use of $\mathrm{TH} 302$ in combination with radiotherapy in patients with esophageal cancer. In this setting HX4 PET imaging will be used to monitor the effect of the treatment. Also, preclinical trials are performed investigating radiotherapy dose escalation based on HX4 PET imaging, in comparison to FDG-PET imaging. The information from these studies will be essential for the clinical implementation of hypoxia boost trials. Last, the use of hypoxia PET imaging in the RADIOMICS project will be started in the near future, which will show us if hypoxia PET imaging provides additional information to the image features derived from standard CT (and FDG-PET) imaging for response prediction.

To summarize, this thesis provides valuable information on the use of hypoxia HX4 PET imaging. The implementation of hypoxia PET imaging in our clinical trial setting is optimized and based on the results of this thesis new initiatives are taken, with the aim to improve our cancer treatment. This thesis was therefore an important step to individualized cancer treatment. 

CURRICULUM VITAE 



\section{CURRICULUM VITAE}

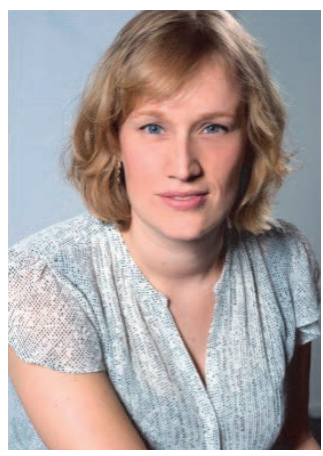

Karen Zegers werd geboren op 30 april 1984 in Heerlen. Zij voltooide in 2002 het VWO op scholengemeenschap Sophianum te Gulpen en vervolgde haar studie met de opleiding Pedagogische Wetenschappen aan de Radboud Universiteit in Nijmegen. $\mathrm{Na}$ twee jaar besloot zij haar focus te verleggen en te starten met de opleiding Technische Geneeskunde aan de Universiteit Twente in Enschede. De specialisatie van haar Master opleiding was medische robotica en beeldvorming. Na diverse stages binnen de disciplines radiologie, chirurgie en elektrofysiologie voltooide zij in 2010 haar afstudeeropdracht 'dosimetrie voor radio-immunotherapie' op de afdeling nucleaire geneeskunde van het Radboud Universitair Medisch Centrum in Nijmegen. Binnen deze afdeling bleef zij nog drie maanden in dienst als onderzoeksassistent. In januari 2011 startte zij met haar promotie onderzoek bij MAASTRO clinic, waar ze in 2014 de Maastro Research Award won. Vanaf juni 2015 is zij werkzaam als klinisch technoloog binnen de groep Fysica Innovatie van Maastro Clinic. 



\section{LIST OF PUBLICATIONS}





\section{LIST OF PUBLICATIONS}

Zegers CM, van Elmpt W, Szardenings K, Kolb H, Waxman A, Subramaniam RM, Moon DH, Brunetti JC, Srinivas SM, Lambin P, Chien D. Repeatability of hypoxia PET imaging using $\left[{ }^{18} \mathrm{~F}\right] \mathrm{HX} 4$ in lung and head and neck cancer patients: a prospective multicenter trial. Eur J Nucl Med Mol Imaging. 2015 Nov;42(12):1840-9.

Lambin P, Zindler J, Vanneste B, van de Voorde L, Jacobs M, Eekers D, Peerlings J, Reymen B, Larue RT, Deist TM, de Jong EE, Even AJ, Berlanga AJ, Roelofs E, Cheng Q, Carvalho S, Leijenaar RT, Zegers CM, van Limbergen E, Berbee M, van Elmpt W, Oberije C, Houben R, Dekker A, Boersma L, Verhaegen F, Bosmans G, Hoebers F, Smits K, Walsh S. Modern clinical research: How rapid learning health care and cohort multiple randomised clinical trials complement traditional evidence based medicine. Acta Oncol. 2015 Oct;54(9):1289300.

Zegers CM, van Elmpt W, Hoebers FJ, Troost EG, Öllers MC, Mottaghy FM, Lambin P. Imaging of tumour hypoxia and metabolism in patients with head and neck squamous cell carcinoma. Acta Oncol. 2015 Oct;54(9):1378-84

Wack LJ, Mönnich D, van Elmpt W, Zegers CM, Troost EG, Zips D, Thorwarth D. Comparison of $\left[{ }^{18} \mathrm{~F}\right]$-FMISO, $\left[{ }^{18} \mathrm{~F}\right]$-FAZA and $\left[{ }^{18} \mathrm{~F}\right]-\mathrm{HX} 4$ for PET imaging of hypoxia - a simulation study. Acta Oncol. 2015 Oct;54(9):1370-7.

Rekers NH, Zegers CM, Yaromina A, Lieuwes NG, Biemans R, Senden-Gijsbers BL, Losen M, Van Limbergen EJ, Germeraad WT, Neri D, Dubois L, Lambin P. Combination of radiotherapy with the immunocytokine L19-IL2: Additive effect in a NK cell dependent tumour model. Radiother Oncol. 2015 Sep;116(3):438-42.

van Elmpt W, Zegers CM, Reymen B, Even AJ, Dingemans AC, Oellers M, Wildberger JE, Mottaghy FM, Das M, Troost EG, Lambin P. Multiparametric imaging of patient and tumour heterogeneity in non-small-cell lung cancer: quantification of tumour hypoxia, metabolism and perfusion. Eur J Nucl Med Mol Imaging. 2015

Dubois LJ, Niemans R, van Kuijk SJ, Panth KM, Parvathaneni NK, Peeters SG, Zegers CM, Rekers NH, van Gisbergen MW, Biemans R, Lieuwes NG, Spiegelberg L, Yaromina A, Winum JY, Vooijs M, Lambin P. New ways to image and target tumour hypoxia and its molecular responses. Radiother Oncol. 2015 Sep;116(3):352-7.

Even AJ, van der Stoep J, Zegers CM, Reymen B, Troost EG, Lambin P, van Elmpt W. PETbased dose painting in non-small cell lung cancer: Comparing uniform dose escalation with boosting hypoxic and metabolically active sub-volumes. Radiother Oncol. 2015 Aug;116(2):281-6.

Peeters SG, Zegers CM, Biemans R, Lieuwes NG, van Stiphout RG, Yaromina A, Sun JD, Hart $\mathrm{CP}$, Windhorst $\mathrm{AD}$, van Elmpt W, Dubois LJ, Lambin P. TH-302 in Combination with Radio- 
therapy Enhances the Therapeutic Outcome and Is Associated with Pretreatment $\left[{ }^{18} \mathrm{~F}\right] \mathrm{HX} 4$ Hypoxia PET Imaging. Clin Cancer Res. 2015 Jul 1;21(13):2984-92.

Rekers NH, Zegers CM, Germeraad WT, Dubois L, Lambin P. Long-lasting antitumor effects provided by radiotherapy combined with the immunocytokine L19-IL2. Oncoimmunology. 2015 Apr 2;4(8)

Zegers CM*, Rekers NH*, Quaden DH, Lieuwes NG, Yaromina A, Germeraad WT, Wieten L, Biessen EA, Boon L, Neri D, Troost EG, Dubois LJ, Lambin P. Radiotherapy combined with the immunocytokine L19-IL2 provides long-lasting antitumor effects. Clin Cancer Res. 2015 Mar 1;21(5):1151-60.

Peeters SG*, Zegers CM*, Yaromina A, Van Elmpt W, Dubois L, Lambin P. Current preclinical and clinical applications of hypoxia PET imaging using 2-nitroimidazoles. Q J Nucl Med Mol Imaging. 2015 Mar;59(1):39-57.

Peeters SG, Zegers CM, Lieuwes NG, van Elmpt W, Eriksson J, van Dongen GA, Dubois L, Lambin P. A comparative study of the hypoxia PET tracers $\left[{ }^{18} \mathrm{~F}\right] H X 4,\left[{ }^{18} \mathrm{~F}\right] \mathrm{FAZA}$, and $\left[{ }^{18}\right.$ F]FMISO in a preclinical tumor model. Int J Radiat Oncol Biol Phys. 2015 Feb 1;91(2): 351-9.

Zegers CM, van Elmpt W, Reymen B, Even AJ, Troost EG, Ollers MC, Hoebers FJ, Houben $\mathrm{RM}$, Eriksson J, Windhorst $\mathrm{AD}$, Mottaghy FM, De Ruysscher D, Lambin P. In vivo quantification of hypoxic and metabolic status of NSCLC tumors using $\left[{ }^{18} \mathrm{~F}\right] \mathrm{HX} 4$ and $\left[{ }^{18} \mathrm{~F}\right] \mathrm{FDG}-\mathrm{PET} / \mathrm{CT}$ imaging. Clin Cancer Res. 2014 Dec 15;20(24):6389-97.

Rekers NH, Troost EG, Zegers CM, Germeraad WT, Dubois LJ, Lambin P. Stereotactic ablative body radiotherapy combined with immunotherapy: present status and future perspectives. Cancer Radiother. 2014 Oct;18(5-6):391-5.

van Elmpt W, Zegers CM, Das M, De Ruysscher D. Imaging techniques for tumour delineation and heterogeneity quantification of lung cancer: overview of current possibilities. J Thorac Dis. 2014 Apr;6(4):319-27.

Zegers CM, Peeters SG, Dubois L, Lambin P Patiëntspecifieke behandeling met behulp van hypoxie PET. Oncology News International. 2014 Feb;8(1)

van Elmpt W, Das M, Hüllner M, Sharifi H, Zegers CM, Reymen B, Lambin P, Wildberger JE, Troost EG, Veit-Haibach P, De Ruysscher D. Characterization of tumor heterogeneity using dynamic contrast enhanced CT and FDG-PET in non-small cell lung cancer. Radiother Oncol. 2013 Oct;109(1):65-70.

Zegers CM, van Elmpt W, Wierts R, Reymen B, Sharifi H, Öllers MC, Hoebers F, Troost EG, Wanders R, van Baardwijk A, Brans B, Eriksson J, Windhorst B, Mottaghy FM, De Ruysscher $\mathrm{D}$, Lambin P. Hypoxia imaging with $\left[^{18} \mathrm{~F}\right] \mathrm{HX} 4$ PET in NSCLC patients: defining optimal imaging parameters. Radiother Oncol. 2013 Oct;109(1):58-64. 
Lambin P, Roelofs E, Reymen B, Velazquez ER, Buijsen J, Zegers CM, Carvalho S, Leijenaar RT, Nalbantov G, Oberije C, Scott Marshall M, Hoebers F, Troost EG, van Stiphout RG, van Elmpt W, van der Weijden T, Boersma L, Valentini V, Dekker A. 'Rapid Learning health care in oncology' - an approach towards decision support systems enabling customised radiotherapy'. Radiother Oncol. 2013 Oct;109(1):159-64.

Zegers CM, Reymen B, Buijsen J, van Stiphout R, Peeters S, Lammering G, Hoebers F, Troost $\mathrm{E}$, van Baardwijk A., Ollers $\mathrm{M}$, van Elmpt W, Lambin P. De bijdrage van PETbeeldvorming in radiotherapie. Kankerbreed 2012 Dec;4(4)

Lambin P, Rios-Velazquez E, Leijenaar R, Carvalho S, van Stiphout RG, Granton P, Zegers CM, Gillies R, Boellard R, Dekker A, Aerts HJ. Radiomics: extracting more information from medical images using advanced feature analysis. Eur J Cancer. 2012 Mar;48(4):441-6.

Stillebroer AB*, Zegers $\mathrm{CM}^{*}$, Boerman OC, Oosterwijk E, Mulders PF, O'Donoghue JA, Visser EP, Oyen WJ. Dosimetric analysis of ${ }^{177}$ Lu-cG250 radioimmunotherapy in renal cell carcinoma patients: correlation with myelotoxicity and pretherapeutic absorbed dose predictions based on ${ }^{111}$ In-cG250 imaging. J Nucl Med. 2012 Jan;53(1):82-9.

* indicates equal contribution 

DANKWOORD 



\section{DANKWOORD}

Een van de genoegens van het voltooien van mijn proefschrift is het terug blikken op de reis en herinneren hoe vele collega's, vrienden en familie me door de jaren heen hebben gesteund.

Allereerst wil ik mijn dank uitspreken aan mijn promotor Prof. Dr. Philippe Lambin en copromotoren Dr. Wouter van Elmpt and Dr. Ludwig Dubois voor alle tijd en energie die zij in mijn promotie hebben gestoken. Philippe wekte mijn interesse voor de wetenschap en stond daarmee aan het begin van mijn wetenschappelijke carrière. Hij bood veel ervaring en onmisbare hulp in de structurering van het onderzoek waardoor ik mij heb kunnen ontwikkelen tot een zelfstandig onderzoeker. Wouter, je deur stond altijd voor me open, waar ik veelvuldig gebruik van gemaakt heb. Bedankt voor je betrokkenheid en waardevolle feedback, onze gesprekken over het onderzoek gaven mij vaak nieuwe energie. Ludwig, je enthousiasme werkt aanstekelijk, bedankt voor je supervisie en kennis, welke een bron van inspiratie voor mij zijn geweest.

Graag wil ik ook de leden van de beoordelingscommissie bedanken (Prof. Dr. F. Ramaekers, Prof Dr. M. Vooijs, Prof Dr. D. Zips en Dr. J. Bussink) voor het lezen en kritisch beoordelen van mijn thesis.

Dit proefschrift bevat data van meerdere klinische studies, welke tot stand zijn gekomen met de hulp van vele mensen. In het bijzonder wil ik alle patiënten bedanken die deelgenomen hebben aan de studies. Daarnaast dank aan alle medewerkers die zich ingezet hebben in de acquisitie van de data. Het datamanagement van Maastro Clinic, met in het bijzonder Anita, ik heb fijne herinneringen aan onze samenwerking m.b.t. de HX4-hoofd hals studie. Trots ben ik dan ook dat we deze studie samen succesvol hebben kunnen afsluiten. Ook speciale dank aan de trial-poli van Maastro, John en Claudia, jullie bijdrage is onmisbaar in de klinische studies binnen Maastro. Veel dank voor jullie support. Ook wil ik mijn dank uitspreken naar alle PET-CT laboranten die in de avonduren patiënten gescand hebben. In het bijzonder Leo en Marlies bedankt voor al jullie raad en daad bij de PET-CT. Dank ook aan de groep klinische fysica, Lars en Michel bedankt voor jullie bijdrage in het opzetten en uitvoeren van de klinische studies. Als laatste wil ik de collega's van de nucleaire geneeskunde bedanken voor de prettige samenwerking. Renee, Christian, Roel, Ivo, Stefan en Felix, bedankt dat ik altijd bij jullie terecht kon.

Tijdens mijn promotie heb ik ook met veel plezier in het Maastro Lab gewerkt. Ik wil alle medewerkers van het lab bedanken voor de gastvrijheid en gezelligheid waarmee ik ontvangen ben. Ik heb ontzettend veel van jullie geleerd. Sarah, jouw werk is van onschatbare waarde geweest voor de totstandkoming van dit proefschrift. Bedankt voor de leuke en gezellige samenwerking. Ik wens je heel veel plezier en succes in je verdere carrière in Oxford. Natasja, jij hebt mij de praktische aspecten van het labwerk geleerd. Je rust en geduld hebben mij het zelfvertrouwen gegeven om zelfstandig in het lab te werken. Nicolle, helaas heeft het pronkstuk van onze samenwerking geen plekje gekregen in dit proefschrift, maar ik ben trots dat het artikel wel in jouw proefschrift zal verschijnen. We 
zijn samen een goed team gebleken op zowel de werkvloer als de dansvloer. Ik wens je veel succes met het afronden van je promotie.

Hartelijk dank aan al mijn coauteurs, voor het tot stand komen van de manuscripten. In het bijzonder, Frank, bedankt voor al je begeleiding en advies bij het opzetten en uitvoeren van de hoofd-hals studie, ik heb met veel plezier met je samen gewerkt. Dirk en Bart, bedankt dat ik direct kon meewerken en gebruik maken van de data verkregen in de long studies. Jullie input kwam de kwaliteit van de artikelen zeker ten goede. Esther, bedankt voor je enthousiasme en waardevolle input in de revisie van de manuscripten. Judith, bedankt voor de analyse van de bloedsamples. Katrin and David, thank you for the opportunity to work together, perseverance always wins, I am proud of the final result.

Speciale dank gaat uit naar mijn paranimfen, Skadi en Sara. Ik vind het een eer dat jullie op 9 maart letterlijk en figuurlijk achter mij staan. Met veel plezier denk ik terug aan onze tijd bij Maastro, we hebben veel beleefd samen en ik hoop dat er nog veel mooie momenten zullen volgen. Skadi, Geert en Olav, heel veel plezier met jullie drietjes, ik wens jullie een hele mooie toekomst samen. Sara, thank you for organizing all the fun shopping trips and Christmas lunches. Obrigado o sucesso no futuro e se divertir com Nuno em Londres. Hoda and Ruud, the last angel and our Charlie, you're both out of sight however not out of my mind. I hope we will all meet again.

A big 'thank you!' to all my fellow researchers. Patrick, Guillaume, Emmanuel, Skadi, Marco, Ruud, Georgi, Daniela, Fiere, Sara, Shane, Evelyn, Aniek, Ralph, Esther, Ruben, Jurgen, Timo, Stefan, Gabriel, Mark, Lotte, Isabel, Sean, Adriana, Lucas, Daan, Davide, Francesco, Hoda, Celine, Pouya, Scott, Arthur, Bregtje, Pedro, Raghu, Mathieu, Abir, Ester, Qing, Janita, Nicolle, Dana, Sarah, Sanaz, Marike, Kranthi, Arjan, Tessa. All of you and the dynamics of the group made me come to work each day with pleasure. I enjoyed our fun discussions and have great memories of the humorous moments in the 'big room', our cheerful lunches, awesome conference visits and special moments in the night life of Maastricht. Good luck with your next paper!

Graag wil ik mijn lieve (schoon)familie en vrienden bedanken voor al het meeleven met belangrijke en minder belangrijke momenten in mijn leven. Leonie en Theo, Sybilla en Rick: bedankt voor jullie interesse en steun, ik wens jullie alvast heel veel plezier met jullie aankomende wondertje. Ron en Tessa, goed voorbeeld doet goed volgen. Bedankt voor jullie adviezen en veel geluk in nabije toekomst met jullie kleine prinsje of prinsesje. Lieve pap en mam, bedankt voor al jullie vertrouwen, raad en daad, en onvoorwaardelijke steun!

Als laatste wil ik de twee belangrijkste mannen in mijn leven bedanken. Philip, lieve sjat, we hebben de afgelopen jaren veel moois meegemaakt. Bedankt dat je er altijd voor me was, met al je liefde, steun en humor. Jij krijgt me altijd weer met beide benen op de grond. Ik weet zeker dat er een mooie toekomst voor ons in het verschiet ligt. Quin, je bent zo klein, maar hebt nu al de grootste veranderingen in mij teweeg gebracht. Er is een titel die meer waard is dan mijn doctoraat, namelijk dat ik mij jouw moeder mag noemen. 


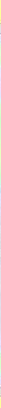

